\title{
INDUCTION OF ANTI-TUMOUR IMMUNE RESPONSES BY DENDRITIC CELLS GENERATED WITH FIt3-LIGAND
}

By

So Nai Lim

\begin{abstract}
A thesis
submitted to the Victoria University of Wellington in fulfilment of the requirements for the degree of

Master of Science in

Cell \& Molecular Bioscience
\end{abstract}
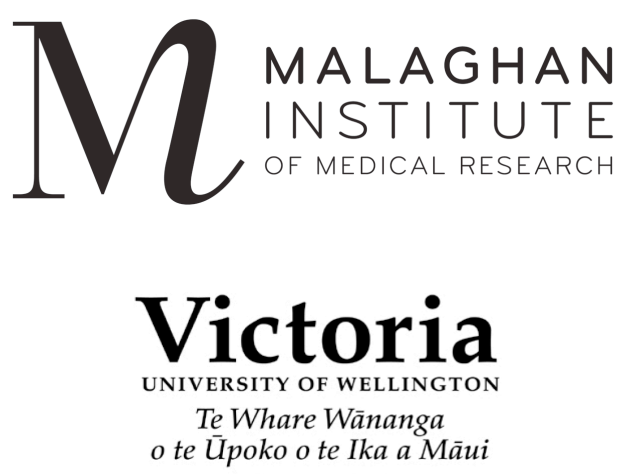

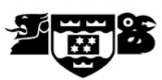

Victoria University of Wellington 


\section{Abstract}

Dendritic cells (DCs) are potent antigen presenting cells that are crucial for the initiation of an immune response. Due to this property, DCs have been used as the basis of cancer vaccines in immunotherapy. In clinical trials, DCs used for vaccination are commonly generated by culturing monocytes from each patients' blood with the growth factors GM-CSF and IL-4 (GMCSF/IL-4 DCs). The DCs generated are reportedly similar to those that arise in vivo during inflammation and trials using these DCs have been met with some success. A recently developed method of generating mouse or human DCs in vitro, involves the culture of bone marrow (BM) precursors with the cytokine Flt3-Ligand (Flt3L-DCs). Flt3L-DCs differ substantially in phenotype from GMCSF/IL-4 DCs and more closely resemble steady-state DCs in vivo. This thesis investigated the suitability of Flt3L-DCs for cancer immunotherapy.

Murine BM cells cultured in Flt3L generated three DC subsets. These consisted of plasmacytoid DCs (pDCs) that were CD11 ${ }^{+} \mathrm{B} 220^{+}$, and conventional DCs (cDCs) that were $\mathrm{CD} 11 \mathrm{c}^{+} \mathrm{B} 220^{-}$and could be further subdivided into $\mathrm{CD} 11 \mathrm{~b}^{\text {high }}$ and $\mathrm{CD} 24^{\text {high }}$ populations. We observed that $\mathrm{cDCs}$ responded to stimulation with a variety of Tolllike receptor (TLR) agonists, as evaluated by the up-regulation of activation markers. However pDCs responded to the agonist $\mathrm{CpG}$ at a higher extent compared to all other agonists used. In addition, combining TLR agonists could further enhance the activation of Flt3L-DCs. Among all combinations tested, Pam3Cys/Poly I:C was the most optimal at inducing the secretion of inflammatory cytokines IL-12p70 and TNF- $\alpha$. Furthermore, Pam3Cys/Poly I:C stimulated Flt3L-cDCs exhibited a greater ability at inducing $\mathrm{CD}^{+} \mathrm{T}$ cell proliferation and cross-presentation of soluble antigen to $\mathrm{CD}^{+} \mathrm{T}$ cells, compared to Flt3L-cDCs activated with the respective individual agonists.

Studies have shown that GM-CSF DCs are highly reliant on glycolytic metabolism during activation in order to up-regulate activation markers. Therefore, we also characterised Flt3L-cDCs for their ability to up-regulate activation markers following stimulation with the agonist LPS and treatment with the glycolysis 
inhibitor 2-Deoxy-D-glucose (2-DG). In line with previous reports, DCs generated in culture with GMCSF/IL-4 were unable to up-regulate activation markers at all the 2-DG concentrations used. In contrast, Flt3L-cDCs appeared to have a threshold level where only high concentrations of 2-DG inhibited their ability to up-regulate activation markers. This result indicates that steady-state and inflammatory DCs preferentially use different metabolic pathways upon activation.

The ability of optimally activated Flt3L-cDCs and GMCSF/IL-4 DCs to confer tumour protection was also examined. While unstimulated Flt3L-cDCs or GMCSF/IL-4 DCs could protect mice from tumour growth, vaccination with activated DCs from either population was required for complete tumour protection. Furthermore, we found that even in optimal conditions of activation, $1 \times 10^{5}$ Flt3LcDCs were required for maximal tumour protection, whereas $1 \times 10^{4}$ GMCSF/IL-4 DCs provided sufficient protection. These findings indicate that Flt3L-cDCs can be used as the basis of a therapeutic cancer vaccine, but are not superior to GMCSF/IL4 DCs. Further studies are required to establish conditions that can enhance the efficacy of Flt3L-cDCs. 


\section{Acknowledgements}

First, I would like to express my sincere gratitude to my supervisor Prof Franca Ronchese for her endless support, patience and guidance. This master's thesis would not have been possible without her acceptance of me as her student and for that I am truly indebted to her.

I am very grateful to Evelyn Hyde for her patience and perseverance in teaching me all the experimental techniques, and to Mark Yang for his advice with protocols. I also wish to thank a past member of the immune cell biology group, Dr Joel Ma, for his help with experiments, encouragement, and friendship.

I am immensely grateful to Stefanie Steiger for her support during my period of writing this thesis, for her supply of German chocolates and Indian curries, and of course for her friendship. Thank you also to Sabine Kuhn for her constant support and encouragement throughout my time at the Malaghan Institute. For my outlets of stress and angst, and for the general support needed and during the course of my masters, thank you to Jessica Field and Taryn Osmond.

I would like to gratefully acknowledge Te Ropu Awhina Putaiao, and in particular Liz Richardson, who encouraged me to aim high and to never underestimate my potential. Her words of wisdom and continued support have helped me succeed in my masters.

Lastly, and most importantly, I wish to thank my parents, Sai Hein and Kim Chheang for their unconditional love, sacrifice, encouragement and support throughout my many years of study. They have been my pillar of strength and motivation. And I am extremely grateful to my brothers and in particular my sister So Boi for the invaluable companionship and for being my never-ending source of humour. 


\section{Table of contents}

$\begin{array}{lll}\text { Abstract } & \text { II }\end{array}$

$\begin{array}{lll}\text { Acknowledgements } & \text { IV }\end{array}$

Table of contents $\quad$ V

List of figures $\quad \mathrm{X}$

List of abbreviations $\quad$ XII

CHAPTER 1. GENERAL INTRODUCTION 1

$\begin{array}{ll}\text { 1.1 TUMOUR IMMUNOLOGY } & 2\end{array}$

1.1.1 CANCER IMMUNOSURVEILLANCE 2

1.1.2 CANCER IMMUNOEDITING 3

1.1.3 TUMOUR ANTIGENS 4

1.1.4 MECHANISMS OF TUMOUR ESCAPE $\quad 4$

1.2 CANCER IMMUNOTHERAPY

1.2.1 POTENTIAL OF IMMUNOTHERAPY 6

$\begin{array}{ll}\text { 1.2.2 DC-BASED IMMUNOTHERAPY } & 7\end{array}$

1.3 DCS AND THE CONTROL OF IMMUNITY 9

1.3.1 DCS ARE PROFESSIONAL ANTIGEN PRESENTING CELLS 9

1.3.2 DC DEVELOPMENT IN VIVO AND REGULATION BY CYTOKINES $\mathbf{1 0}$

$\begin{array}{lr}\text { 1.4 DC SUBSETS } & 12\end{array}$

$\begin{array}{ll}\text { 1.4.1 PLASMACYTOID DCs } & \mathbf{1 3}\end{array}$

$\begin{array}{lr}\text { 1.4.2 CONVENTIONAL DCS } & \mathbf{1 3}\end{array}$

$\begin{array}{lr}1.5 \text { ACTIVATION STATES OF DCS } & 15\end{array}$

1.5.1 IMMATURE DCS $\quad \mathbf{1 5}$

1.5.2 MATURE TOLEROGENIC DCS

1.5.3 MATURE DCs $\quad \mathbf{1 6}$

$\begin{array}{ll}1.6 & \text { DC ACTIVATION THROUGH TOLL-LIKE RECEPTORS }\end{array}$

$\begin{array}{lr}1.7 \text { ANTIGEN PRESENTATION TO T CELLS } & 18\end{array}$

$\begin{array}{ll}\text { 1.7.1 MHC I PRESENTATION } & \mathbf{1 8}\end{array}$

1.7.2 MHC II PRESENTATION

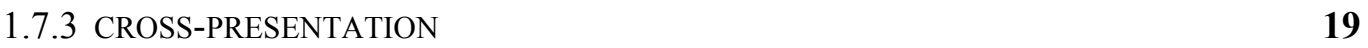


$\begin{array}{lr}1.8 \text { INDUCTION OF T CELL ACTIVATION BY DCS } & 20\end{array}$

1.8.1 $\mathrm{CD}^{+}{ }^{+}$T CELL ACTIVATION AND EFFECTOR FUNCTION $\mathbf{2 1}$

1.8.2 $\mathrm{CD}^{+}{ }^{+}$T CELL ACTIVATION AND EFFECTOR FUNCTION $\mathbf{2 2}$

1.9 THE ROLE OF GM-CSF ANF IL-4 IN GENERATING DCS IN VITRO 23

1.9.1 GM-CSF AND IL-4 ARE COMMONLY USED FOR GENERATING MONOCYTE-DERIVED DCS IN CLINICAL TRIALS

1.9.2 GM-CSF INDUCES THE DEVELOPMENT OF INFLAMMATORY Mo-DCS $\mathbf{2 4}$

1.10 FLT3L IS CRUCIAL FOR NORMAL HEMATOPOIESIS AND DC DEVELOPEMNT IN VIVO

1.11 FLT3L BM CULTURES GENERATE DCS THAT ARE EQUIVALENT TO STEADY-STATE DCS IN VIVO

$\begin{array}{lr}\text { 1.12 OBJECTIVES OF THIS STUDY } & 28\end{array}$

CHAPTER 2. MATERIALS AND METHODS $\quad 29$

2.1 MATERIALS

2.1.1 LABWARE 30

2.1.2 REAGENTS AND MATERIALS 31

2.1.3 CYTOKINES AND GROWTH FACTORS 34

$\begin{array}{ll}2.1 .4 & 35\end{array}$

2.1.5 ANTIBODIES AND FLUOROPHORES 36

2.1.6 PROTEINS AND PEPTIDES

2.1.7 ASSAY BUFFER COMPOSITIONS 39

$\begin{array}{ll}2.1 .8 \text { KITS } & 40\end{array}$

$\begin{array}{ll}\text { 2.2 TUMOUR CELL LINES } & 40\end{array}$

$\begin{array}{ll}2.3 \\ \text { MICE } & 40\end{array}$

2.3.1 MAINTENECE AND ETHICS APPROVAL 40

2.3.2 MOUSE STRAINS 41

2.4 MethodS 41

2.4.1 GENERAL CELL CULTURE 41

2.4.2 DENDRITIC CELL CUTLURING AND MANIPULATION 42

2.4.2.1 EXTRACTION OF BONE MARROW 42

2.4.2.2 GENERATING FLT3L-DCS 42 
2.4.2.3 GENERATING GMCSF/IL-4 DCs 42

2.4.2.4 INDUCING DC ACTIVATION 43

2.4.2.5 LOADING FLT3L-DCS WITH PEPTIDES FOR IN VITRO ASSAYS 43

2.4.2.6 LOADING FLT3L-DCS WITH OVA PROTEIN 44

2.4.2.7 TREAMENT OF FLT3L AND GMCSF/IL-4 DCs WITH 2-DEOXY-D-GLUCOSE 44

2.4.3 CELL PURIFICATION/DEPLETION AND CELL SORTING 44

2.4.3.1 PREPERATION OF LYMPH NODE AND SPLEEN SUSPENSION 44

2.4.3.2 PURIFICATION OF T CELLS USING MAGNETIC CELL SEPARATION 45

2.4.3.3 DEPELTION OF B220 ${ }^{+}$pDCS USING MAGNETIC CELL SEPARATION 45

2.4.3.4 DEPELTION OF B220 ${ }^{+}$pDCS USING BioMag® BEADS 46

2.4.3.5 FLUORESENCE ACTIVATED CELL SORTING (FACS) OF FLT3L-DCS 46

2.4.4 ASSAYS OF CELL FUNCTION

2.4.4.1 IN VITRO T CELL PROLIFERATION ASSAY

2.4.4.2 IN VITRO CROSS-PRESENTATION ASSAY 48

2.3.4.3 DETECTION OF CYTOKINE PRODUCTION FROM DC SUPERNATANT 48

2.4.5 FLUORESCENT LABELLING OF CELLS AND FLOW CYTOMETRY 49

2.4.5.1 DETECTION OF SURFACE MARKER EXPRESSION 49

2.4.5.2 DETECTION OF INTRACELLULAR CYTOKINES 50

2.4.5.3 ACQUISITION AND ANALYSIS $\quad 50$

2.4.6 HISTOLOGY

2.4.6.1 PREPERATION OF DCS FOR MORPHOLOGICAL ANALYSIS AND IMMUNOFLUORESNCE STAINING 51

2.4.6.2 MORPHOLOGICAL ANALYSIS OF DCS 51

2.4.6.3 IMMUNOFLUORESCENCE STAINING OF FLT3L-DCS 51

2.3.6.4 MICROSCOPE IMAGE ACQUISITION AND ANALYSIS 52

2.4.7 DC VACCINATION OR TUMOUR CHALLENGE

2.4.7.1 DC VACCINATION 52

2.4.7.2 CHALLENGE WITH B16.OVA MELANOMA CELLS 53

$\begin{array}{ll}2.5 \text { STATISTICAL ANALYSIS } & 53\end{array}$

CHAPTER 3. CHARACTERISATION OF FLT3L-DCS AND EVALUAION OF THEIR RESPONSE TO STIMULATION WITH TLR AGONISTS

$\begin{array}{ll}3.1 \text { INTRODUCTION } & 55\end{array}$ 
3.1.1 DC ACTIVATION WITH COMBINED TLR AGONISTS 55

3.1.2 METABLOIC REQUIRMENTS FOR CELLULAR ACTIVATION 57

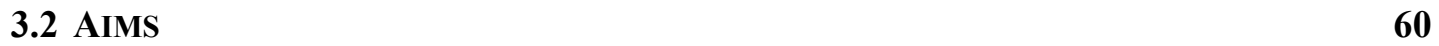

$\begin{array}{ll}3.3 \text { RESULTS } & 60\end{array}$

3.3.1 MORPHOLOGICAL CHARACTERISTICS OF FLT3L-DCS $\quad 60$

3.3.2 PHENOTYPIC CHARACTERISATION OF FLT3L-DCS BY IMMUNOFLUORESCENCE STAINING AND FLOW CYTOMETRY

3.3.3 FLT3L-DC SUBSETS RESPOND TO STIMULATION WITH TLR AGONISTS 65

3.3.4 DEPLETION OF pDCs DOES NOT PREVENT THE RESPONSE OF cDCs TO STIMULATION WITH TLR AGONISTS $\quad 68$

3.3.5 STIMUALTION WITH COMBINED TLR AGONISTS IMPROVES THE MATURATION OF

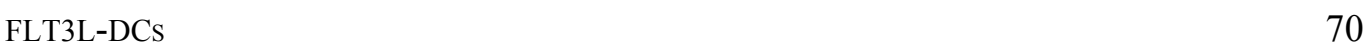

3.3.6 LOW CONCENTRATION OF 2-DG INHIBIT THE MATURATION OF GMCSF/IL-4 DCs BUT

NOT FLT3L-CDCS

$\begin{array}{llr}3.4 & \text { DISCUSSION } & \mathbf{8 2}\end{array}$

3.4.1 PHENOTYPIC AND MORPHOLOGICAL CHARACTERISTICS OF FLT3L-DCS 82

3.4.2 RESPONSE OF FLT3L-DCS TO STIMULATION WITH INDIVIDUAL TLR AGONISTS 83

3.4.3 THE IMPACT OF COMBINED TLR LIGATION ON THE MATURATION OF FLT3L-DCS 84

3.4.4 REGULATION OF DC ACTIVATION BY GLUCOSE METABOLISM 85

$\begin{array}{llr}3.5 & \text { CONCLUSION } & 87\end{array}$

CHAPTER 4. DETERMINING THE FUCNTIONAL CAPABILITIES OF FLT3L\begin{tabular}{lr} 
DCS & 89 \\
\hline
\end{tabular}

$\begin{array}{lr}4.1 \text { INTRODUCTION } & 90\end{array}$

4.1.1 ANTI-TUMOUR IMMUNE RESPONSE REQUIRES CD $4^{+}$AND CD8 ${ }^{+}$T CELL ACTIVATION 90

4.1.2 THE ROLE OF CYTOKINES IN ANTI-TUMOUR IMMUNE RESPONSE 91

$\begin{array}{lr}4.2 & 91 M S \\ \end{array}$

$\begin{array}{lr}4.3 \text { RESULTS } & 92\end{array}$

4.3.1 ACTIVATION OF FLT3L-DCS WITH INDIVIDUAL TLR AGONISTS INDUCES CYTOKINE PRODUCTION 92

4.3.2 SELECTED TLR AGONIST COMBINATIONS ENHANCE THE SECRETION OF PROINFLAMMATORY CYTOKINES 94 
4.3.3 FLT3L-DC SUBSETS DIFFER IN THEIR CAPACITY TO SECRETE PRO-INFLAMMATORY CYTOKINES

4.3.4 STIMULATION WITH COMBINED TLR AGONISTS IMPROVES THE ABILITY OF FLT3LDCS TO INDUCE $\mathrm{CD}^{+}{ }^{+} \mathrm{T}$ CELL PROLIFERATION

4.3.5 FLT3L-DCs STIMULATED WITH THE COMBINED TLR AGONISTS ARE BETTER AT CROSS-PRESENATION

4.3.6 VACCINATION WITH 1 X10 $0^{5}$ FLT3L-CDCS CAN INDUCE TUMOUR PROTECTION IN MICE

4.3.7 DISRUPTION OF FLT3L-DC CLUSTERS PRIOR TO STIMULATION WITH TLR AGONISTS DOES NOT AFFECT TUMOUR PROTECTION

4.3.8 UNSTIMULATED AND STIMULATED FLT3L-CDCS EXHIBIT SIMILAR LEVELS OF TUMOUR PROTECTION

\subsection{DisCUSSION}

4.4.1 CYTOKINE SECRETION PROFILES OF FLT3L-DCS FOLLOWING STIMULATION WITH THE INDIVIDUAL TLR AGONISTS

4.4.2 DIFFERENTIAL CAPACITY OF CYTOKINE SECRETION FROM FLT3L-DCS SUBSETS

4.4.3 ENHANCED PROLIERATION OF CD4 ${ }^{+}$T CELLS BY FLT3L-CDCS STIMULATED WITH THE COMBINED TLR AGONISTS

4.4.4 ENHANCED CROSS-PRESENATION OF SOLUBLE ANTIGEN BY FLT3L-cDCs STIMULATED WITH THE COMBINED TLR AGONISTS 


\section{List of figures}

Figure 1.1: General strategy employed in DC-based vaccination.

Figure 1.2: DC development from hematopoietic precursors.

Figure 3.1: Overview of the TLR agonists used within this study and the corresponding signalling pathways.

Figure 3.2: Resting and activated $\mathrm{T}$ cells display distinct metabolic signatures.

Figure 3.3: Morphology of Flt3L-DCs.

Figure 3.4: Detection of Flt3L-DC subsets by immunofluorescence staining.

Figure 3.5: The culture of $\mathrm{BM}$ cells in Flt $3 \mathrm{~L}$ generates three $\mathrm{DC}$ subsets.

Figure 3.6: Flt3L-DC subsets display a trend to activation upon TLR stimulation.

Figure 3.7: cDCs are capable of maturation in the absence of pDCs.

Figure 3.8: Combined TLR stimulation enhances Flt3L-DC maturation.

Figure 3.9: Stimulating Flt3L-DCs with TLR agonist combinations does not reduce cell viability.

Figure 3.10: Treatment with LPS induces the maturation of Flt3L and GMCSF/IL-4 DCs following a 6 hour stimulation.

Figure 3.11: Low concentrations of 2-DG have limited effects on the maturation of Flt3L-cDCs induced by LPS, but strongly affects the maturation of GMCSF/IL-4 DCs.

Figure 4.1: Flt3L-DCs activated with TLR agonists produce pro-inflammatory and anti-inflammatory cytokines.

Figure 4.2: Selected TLR agonist combinations enhance the secretion of pro-inflammatory cytokines from Flt3L-DCs. 
Figure 4.3: Production of IL-12p40 is detected in cDCs, but not in pDCs. $\quad 98$

Figure 4.4: TNF- $\alpha$ production is only detected in CD2 $4^{\text {high }}$ cDCs.

Figure 4.5: Flt3L-cDCs stimulated with combined TLR agonists acquire greater capabilities to induce proliferation of $\mathrm{CD} 4^{+}$but not $\mathrm{CD} 8^{+} \mathrm{T}$ cells.

Figure 4.6: Combined TLR ligation enhances the capability of Flt3L-cDCs to cross-present OVA.

Figure 4.7: Vaccination with graded numbers of Flt3L-cDCs and GMCSF/IL-4 DCs can induce tumour protection in mice.

Figure 4.8: Vaccination with Flt3L-DCs or Flt3L-cDCs induces similar levels of tumour protection.

Figure 4.9: Stimulated and unstimulated Flt3L-cDCs do not differ substantially in their ability to protect mice from tumour growth. 


\section{List of abbreviations}

\begin{tabular}{|c|c|}
\hline 2-DG & 2-Deoxy-D-Glucose \\
\hline $2 \mathrm{ME}$ & 2 mercaptoethanol \\
\hline $\mathrm{ACT}$ & Ammonium Chloride Tris \\
\hline $\mathrm{APC}$ & Antigen-presenting cell \\
\hline ATP & Adenosine triphosphate \\
\hline $\mathrm{BM}$ & Bone marrow \\
\hline BM-DC & Bone marrow-derived dendritic cell \\
\hline BSA & Bovine serum albumin \\
\hline $\mathrm{CCR}$ & $\mathrm{CC}$ chemokine receptor \\
\hline $\mathrm{CD}$ & Cluster of differentiation, cluster of designation \\
\hline $\mathrm{cDC}$ & Conventional dendritic cell \\
\hline cIMDM & Complete IMDM \\
\hline CLP & Common lymphoid progenitor \\
\hline CLIP & Class II-associated invariant chain peptide \\
\hline CMP & Common Myeloid progenitor \\
\hline CTL & Cytotoxic T lymphocytes \\
\hline DAPI & 4',6-diamidino-2-phenylindole \\
\hline DC & Dendritic cell \\
\hline DN DC & Double negative dendritic cell \\
\hline DNA & Deoxyribonucleic acid \\
\hline dsRNA & Double-stranded RNA \\
\hline EDTA & Ethylendiaminetetraacetic Acid \\
\hline ER & Endoplasmic reticulum \\
\hline EtOH & Ethanol \\
\hline FACS & Fluorescence activated cell sorting \\
\hline FBS & Foetal Bovine Serum \\
\hline FITC & Fluorescein isothiocyanate \\
\hline Flt3 & Fms-like tyrosine kinase 3 \\
\hline Flt3L & Fms-like tyrosine kinase 3 ligand \\
\hline FSC & Forward Scatter \\
\hline GLUT1 & Glucose transporter 1 \\
\hline
\end{tabular}


GM-CSF

GM-CSFR

G-6-P

HSC

IFN $\gamma$

IgG

IL

iNOS

IRF

IMDM

i.p.

i.v.

LC

LDFB

LN

LPS

$\mathrm{mAB}$

MAGE

MACS

M-CSF

MDA

Melan-a

MFI

MHC II

Mo-DC

mRNA

$\mathrm{NAD}^{+}$

NF-кB

NK cells

OVA

PAMP

PBS

PE
Granulocyte macrophage colony-stimulating factor

GM-CSF receptor

Glucose-6-phosphate

Haematopoietic stem cell

Interferon gamma

Immunoglobulin G

Interleukin

Inducible Nitric Oxide Synthase

Interferon-regulated factor

Iscoves modified dulbeco's medium

Intraperitoneal

Intravenous

Langerhans cells

Live Dead Fixable Blue

Lymph node

Lipopolysaccharide

Monoclonal antibody

Melanoma antigen-encoding gene

Magnetic cell separation

Macrophage colony-stimulating factor

3,4-Methylenedioxyamphetamine

Melanocyte lineage antigen (also known as MART-1)

Median fluorescence intensity

Major histocompatibility complex class II molecules

Monocyte-derived dendritic cell

Messenger ribonucleic acid

Nicotinamide adenine dinucleotide

Nuclear factor kappa-light-chain-enhancer of activated B cells

Natural Killer cells

Ovalbumin

Pathogen-associated molecule pattern

Phosphate Buffered Saline

Phycoerythrin 


\begin{tabular}{|c|c|}
\hline PerCP & Peridinin chlorophyll A protein \\
\hline $\mathrm{pDC}$ & Plasmacytoid dentritic cell \\
\hline PI & Propidium Iodide \\
\hline PPR & Pattern recognition receptor \\
\hline Rag & Recombination activating gene \\
\hline RIG-1 & Retinoic acid-inducible gene 1 \\
\hline RLRs & RIG-1-like receptors \\
\hline SA & Streptavidin \\
\hline s.c. & Subcutaneous \\
\hline S.D. & Standard deviation \\
\hline SIINFEKL & Ovalbumin MHC class I peptide \\
\hline $\mathrm{SSC}$ & Side Scatter \\
\hline ssRNA & Single-stranded RNA \\
\hline TAA & Tumour-associated antigen \\
\hline TAP & Transporter of antigen-processing \\
\hline TGF $\beta$ & Transforming growth factor beta \\
\hline Th & T helper \\
\hline TSA & Tumour-specific antigen \\
\hline TRP-2 & Tyrosinase-related protein-2 \\
\hline Tregs & Regulatory T cells \\
\hline TAM & Tumour-associated macrophages \\
\hline TLR & Toll-like receptor \\
\hline TNF $\alpha$ & Tumour growth factor \\
\hline TCR & $\mathrm{T}$ cell receptor \\
\hline$\left[{ }^{3} \mathrm{H}\right]$-thymidine & Tritiated thymidine \\
\hline TRIF & TIR-domain-containing adapter-inducing interferon- $\beta$ \\
\hline VEGF & Vascular-endothelial growth factor \\
\hline
\end{tabular}


Chapter 1.

General Introduction 


\subsection{Tumour immunology}

\subsubsection{Cancer immunoserveillance}

In the early 1900's, Paul Ehrlich was one of the first to conceive the idea that the immune system can repress the growth of carcinomas (1). However the validity of this prediction could not be experimentally tested at the time due to limited knowledge about the composition and function of the immune system (2). Fifty years later, as the understanding of immunology developed, the idea of immune control over cancer resurfaced. This was largely due to the discovery of tumour antigens, demonstrated by the finding that mice immunised with chemically induced tumours were protected against subsequent rechallenge with the same tumour $(3,4)$. These advances in tumour immunology provided the foundation for Burnet and Thomas to formulate cancer immunoserveillance hypothesis, a concept that envisaged the idea that the immune system can have a protective role in tumour development (5). Unfortunately, subsequent studies using nude mice with spontaneous mutations that rendered them immunocomprimised but not completely immunodeficient, failed to provide evidence for this hypothesis $(5,6)$. Specifically, these mice neither developed increased incidence of chemically induced or spontaneous tumour growth compared to wild-type controls (7). On the basis of these findings, the cancer immunoserveillance hypothesis was largely abandoned until the 1990's when development of knockout technologies provided improved murine models of immunodeficiency (6). Thereafter, more recent studies in mice with defined immunodeficiencies have supported the concept of cancer immunoserveillance (8). Particularly, this was seen in studies of Rag2 knockout mice which lacked $\mathrm{T}$ and $\mathrm{B}$ cells, resulting in a higher susceptibility to chemically induced and spontaneous tumour development $(9,10)$.

Correlative data in humans have also provided compelling evidence of immunoserveillance. This was seen in documented cases of human tumours that are infiltrated by inflammatory cells. Infiltration of inflammatory cells can be taken as evidence that the host's immune system "is not ignorant of the developing tumour but rather attempts to interfere with tumour progression" (11). Indeed reports of immune infiltrates within the literature have correlated with improved prognosis in patients with colorectal and ovarian cancer $(12,13)$. Furthermore, individuals treated with 
immunosuppressive drugs have a greater risk of developing certain kind of cancers, such as squamous cell carcinoma and lymphomas $(14,15)$. Taken together, these findings suggest that the immune system is likely to play a role in tumour suppression.

\subsubsection{Cancer immunoediting}

Despite the existence of cancer immunosurveillance, immunocompetent individuals still develop cancer. This clinical reality was first explained by the work of Shankaran et al, who demonstrated that the immune system not only controls tumour growth, but can also select the outgrowth tumour cells with reduced immunogenicity, capable of escaping immune recognition and destruction (9). This discovery prompted development of the cancer immunoediting hypothesis by Dunn et al which takes into account the hostprotective (cancer immunoserveillance) and tumour-sculpting actions of the immune system $(2,5)$.

Cancer immunoediting is a process that encompasses three main phases: Elimination, Equilibrium and Escape. The elimination process incorporates the original concept of cancer immunoserviellence, whereby cells of the innate (i.e. macrophages, NK cells) and adaptive ( $\mathrm{T}$ and $\mathrm{B}$ cells) immune system work together to detect and destroy nascent transformed cells (16). If elimination proceeds towards completion, than the host remains cancer free. However, if tumour cells are not fully eradicated, they may enter the equilibrium phase whereby cells of the immune system such as B and T cells exert potent immune selection pressure that is enough to contain but not completely destroy the tumour $(5,6)$. This process leads to immune selection of tumour cells with reduced immunogenicity and are thus capable of surviving in an immunocompetent host. The equilibrium phase may likely represent the longest of all three processes, occurring over a period of many years (17). Experimental evidence for the equilibrium phase was provided by Koebel et al, who demonstrated in mice that originally failed to develop tumours after treatment with a low dose of a chemical carcinogen, could develop tumours after treatment with $\mathrm{mAb}$ designed to compromise the immune system (18). Edited tumour cells surviving the equilibrium phase enter the escape process. The surviving tumour variants that have acquired the capacity to evade immunological detection through so called 
"tumour escape mechanisms" begin to expand, thus resulting in clinically observable malignant disease (5). Strategies used by tumours to evade the immune response will be discussed in section 1.1.4.

\subsubsection{Tumour antigens}

Tumour cells often express antigens that are distinct to those of normal cells, and are therefore called tumour antigens. They can be products of mutated cellular genes, aberrantly expressed normal genes or genes encoding viral proteins (6). The first tumour antigen was discovered by examining $T$ cell responses against melanoma cells (19). The antigen identified was MAGE-1 $(20,21)$ and was crucial in the development of tumour immunology by providing evidence of specific interaction of the immune system with antigenic determinants presented by the tumour (19). Depending on their pattern of expression, tumour antigen can be broadly divided into two categories: Tumour-associated antigen (TAA) and tumour-specific antigen (TSA). TAA consists of normal proteins that are aberrantly expressed on tumour cells compared to healthy cells. TSA represents altered self proteins, expressed only in tumour cells and are the product of mutated genes, translocations, or transcription of alternative reading frames (22). Melanomas in particular have been shown to express TAAs, such as TRP-2 (23) and Melan-a/MART-1 (24). The expression of these antigens is found on both melanomas and well as normal melanocytes. An example of TSA is the BCR-ABL fusion protein found in individuals with chronic myeloid leukemia. BCR-ABL is the product of translocation between chromosomes 9 and 22 (25). The identification and characterisation of tumour antigen shows that tumour cells possess distinctive structures that can be recognized by the immune system. Ultimately, this finding has set the stage for the development of targeted methods for the immunotherapy of cancer.

\subsubsection{Mechanisms of tumour escape}

Once established, the tumour microenvironment represents an effective barrier to immune cell functions. This is because tumour cells actively down-regulate anti-tumour immune 
responses using a variety of escape mechanisms.

Tumour cells express specific antigens on the cell surface, which are associated with MHC molecules (26). Accordingly, cytotoxic $\mathrm{CD}^{+} \mathrm{T}$ cells can recognise tumour antigens presented by MHC I molecules and induce tumour killing. However, tumour cells can escape T cell recognition through down-regulation or loss of surface MHC I (19).

Alternatively, cancer cells can produce immosuppressive cytokines such as vascularendothelial growth factor (VEGF), transforming growth factor- $\beta$ (TGF- $\beta$ ) and interleukin 10 (IL-10). VEGF is secreted by many tumours (27) and is a key factor inducing tumour vascularisation $(28,29)$. Furthermore, VEGF has been shown to suppress activation of the transcription factor NF- $\mathrm{KB}$ in hematopoietic stem cells (HSC), thus preventing DC differentiation (30). Blockade of NF-אB activation in HSCs by tumour-derived factors is thought to be a mechanism by which tumour cells down-regulate the ability of the immune system to generate an anti-tumour immune response. TGF- $\beta$ is angiogenic and secretion by tumour cells leads to inhibition of DC activation as well as direct inhibition of $\mathrm{T}$ cell and NK cell function $(31,32)$. Similarly, IL-10 present within tumours can suppress DC function and skew $\mathrm{T}$ cell responses toward a type 2 immune response that is less effective against malignant cells (33)

Tumour cells can also recruit immune cells that function as effectors of immunesuppression, such as regulatory $\mathrm{T}$ cells (Tregs) and tumour-associated macrophages (TAMs). Tregs are critical mediators of peripheral tolerance under physiological settings, but are often recruited to the tumour site where they suppress anti-tumour immunity. Tregs inhibit the function of tumour specific $\mathrm{T}$ cells by production of immunosuppressive cytokines such as IL-10 and TGF- $\beta$ and by expression of inhibitory molecules such as CTLA-4 (34). TAMs can promote tumour growth, by production of potent angiogenic cytokines such as VEGF that regulate tumour angiogenesis (35). Although macrophages from healthy tissues are capable of lysing tumour cells, the cytotoxic activity of TAMs within the tumour microenvironment is deactivated due to production of suppressive cytokines by the tumour (36). Mounting lines of evidence also implicate TAMs in the regulation of metastasis. High numbers of TAMs in primary tumours have been correlated 
with early establishment of metastases in a number of tumour types $(37,38)$. Together, these examples demonstrate that tumour cells exhibit several molecular and cellular mechanisms that contribute to suppression of anti-tumour immunity.

\subsection{Cancer immunotherapy}

\subsubsection{Potential of immunotherapy}

Cancer is the second leading cause of death in the industrialised world (39). To this day, local therapy such as surgical excision and ablation by radiation is a mainstay for the treatment of primary cancer. Although curative for a percentage of patients, over time many will succumb to recurrent diseases or metastasis (40). Chemotherapy maybe used as a sole treatment or in conjunction with radiotherapy to enhance the therapeutic effect. However, chemotherapeutic agents currently used are cytotoxic and affect both normal and malignant cells (41).

The fundamental rational for cancer immunotherapy lies within the potency and specificity of the immune system, which can be harnessed to eliminate tumour cells. High selectivity allows the immune system to mount a powerful attack on the tumour with minimal toxicity to healthy tissue, thereby improving quality of life for patients (42). In 1985, Rosenberg et al was the first to demonstrate that immunological manipulation could result in regression of established, invasive cancer in humans. The study consisted of administrating high doses of the cytokine IL-2 into patients with metastatic melanoma, kidney cancer and nonHodgkin's lymphoma and regression of tumours were observed in selected number of patients (43). IL-2 is secreted by human T-lymphocytes and can exert a full array of immune regulatory effects, including the induction of lymphocyte expansion (44). Reportedly, cancer cells grow unimpeded by high concentrations of IL-2 in vitro, thus indicating that the clinical responses observed in patients administered with IL-2, were due stimulation of T-lymphocytes with anti-tumour activity (44).

Currently, therapeutic intervention to amplify or induce the anti-tumour immune response can be categorised into two main types: passive immunotherapy or active immunotherapy. 
Passive immunotherapy is based on the adoptive transfer of ex vivo activated immune cells, immunomodulators such as cytokines or tumour specific antibodies (45). Active immunotherapy is aimed at activating the patients own immune system via the administration of a therapeutic vaccine $(46,47)$. This strategy has the advantage, in that if successful, can elicit a long-lasting immunological memory that can protect against tumour recurrence. Several approaches of active immunotherapy have been tested so far, the most promising form being the use of DCs as "nature's adjuvant" (48).

\subsubsection{DC-based immunotherapy}

Therapeutic vaccines against cancer aim to induce antigen-specific $\mathrm{T}$ cell responses, which are principal effectors of anti-tumour immunity (49). Owing to their superior ability of antigen presentation and induction of $\mathrm{T}$ cell immunity, DCs are increasingly used for therapeutic manipulation of the immune system (48). The primary goal of DC-based vaccines is to "mend the inattention of the immune system" by vaccination with ex vivo generated DCs appropriately activated and loaded with tumour antigens (50).

Early studies in mice first demonstrated the applicability of DC vaccination for cancer immunotherapy. Because DCs are rare in vivo $(0.3 \%$ of the blood leukocyte population (51)), much of these initial studies in animals were facilitated by techniques for generating large numbers of DCs in vitro. Specifically, DCs can be generated from murine BM cell cultures supplemented with the cytokines GM-CSF and IL-4 (GMCSF/IL-4) to induce DC differentiation (52). BM-derived DCs loaded with tumour peptides and administered as a cellular vaccine have been found to induce protective and therapeutic anti-tumour immunity in mice (53-55). Similarly, murine DCs pulsed with unfractionated tumour lysates have also shown a high degree of success in preventing tumour development (56). Overall, vaccination with antigen-loaded DCs has proven their potency at inducing antitumour immunity in mice, thus prompting the testing of DC-based vaccines in clinical studies.

A common approach of generating DCs for clinical trials is to use CD34 ${ }^{+}$progenitor cells or monocytes that are isolated from the patients' blood by leukapheresis (Figure 1.1). 
These cells are cultured in the presence of various cytokine mixtures to induce DCs differentiation, treated with a maturation stimulus such as the Poly I:C and loaded with tumour antigens (57). The DCs are then administered into the patient with the intention of inducing antigen-specific B and T cell responses $(58,59)$. Vaccination studies have shown that DC vaccines are safe, with only minimal side effects observed (60). Although the clinical benefit that is measured by regression of established tumours has been observed in only a fraction of the patients (48), this nonetheless provides evidence that DC vaccination can be successful in principal.

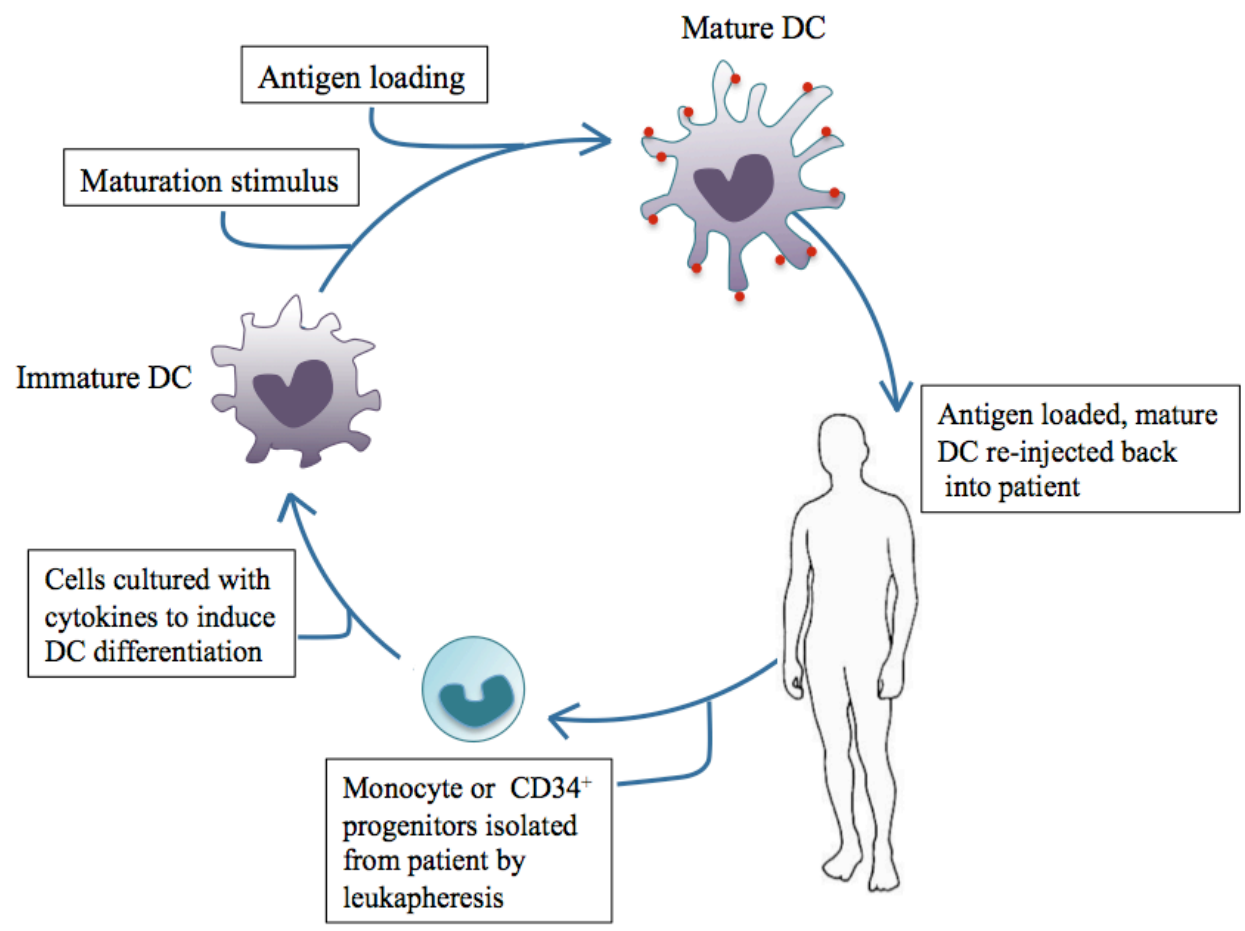

Figure 1.1: General strategy employed in DC-based vaccination.

DC precursors (monocytes or $\mathrm{CD} 34^{+}$progenitor cells) are first obtained by leukapheresis. Precursor cells are then placed in a period of in vitro cell culture with cytokines to induce DC differentiation. The DCs thus generated display an immature phenotype and are induced to mature by a maturation stimulus. DCs are loaded with tumour antigens before being re-injected into the patient as a therapeutic vaccine. Adapted from O’Neil et al (59) and Jefford et al (61). 


\subsection{DCs and the control of immunity}

\subsubsection{DCs are professional antigen presenting cells}

The initiation of a $\mathrm{T}$ cell response against a foreign antigen is driven by antigen uptake and presentation of peptide fragments by antigen-presenting cells (APCs). DCs are potent APCs and are distinct from other less potent APCs such macrophages and B cells (51). B cells are poor at antigen uptake and mainly specialise in antibody production. Furthermore, B cells are inefficient at stimulating naïve $\mathrm{CD}^{+} \mathrm{T}$ cells (62). Conversely, macrophages are highly proficient at antigen uptake, but antigen degradation often destroys the antigenic epitopes on the antigen that are necessary for $\mathrm{T}$ cell activation (63). The biological features that make DCs superior APCs include their capacity of antigen uptake with a corresponding low rate of antigen degradation, allowing for prolonged antigen presentation. Furthermore, DCs are functionally plastic and are located within strategic areas of the body (63). It is these features that define DCs as "professional" APCs.

Distributed as sentinels throughout the body, DCs play a critical role in the induction and regulation of immune responses (64). DCs are a heterogeneous population of cells and there are two main subpopulations found within the steady-state: plasmacytoid DCs (pDC) and conventional DCs (cDC) (65). These two subpopulations can be further subdivided based on phenotype, location and function within the immune system (further discussed in section 1.4). In the immature state, DCs reside in the peripheral tissues, constantly sampling the environment for foreign antigen. In the absence of microbial stimuli or inflammatory cytokines, DCs can spontaneously mature and migrate to the lymph nodes (LN) to present the captured antigen to T cells. Antigen presentation by immature DCs is thought to be a mechanism by which peripheral tolerance to self-antigen is maintained. When DCs encounter microbial products, they become activated and migrate to the LN for antigen presentation to $\mathrm{T}$ cells, resulting in $\mathrm{T}$ cell activation and differentiation into effector cells $(64,66)$. 


\subsubsection{DC development in vivo and regulation by cytokines}

DCs, together with all blood cells originate from pluripotent hematopoietic stem cells (HSC) from the bone marrow (BM) $(67,68)$. All HSC-derived cells develop either as a part of the lymphoid or myeloid pathway, through a multipotent, but lineage-restricted common lymphoid precursor (CLP) or common myeloid precursor (CMP) $(69,70)$ (Figure 1.2). Studies have now provided evidence that DC subsets can be generated through both the myeloid and lymphoid pathways, from CMP or CLP $(71,72)$. The fact that both CMP and CLP can generate all DC populations suggests plasticity in developmental potentials of these early precursors (73). However, the potential to develop into DCs is only found among the fractions of CMP and CLP subpopulations expressing the receptor Flt3 (74) (Figure 1.2). Flt3 (FMS-related tyrosine kinase 3) is a member of the class III receptor tyrosine kinase family (75) that is expressed on early progenitor cells from the BM. Both CMP and CLP precursors have been found to be heterogenous for Flt3 expression, with CLP expressing most of Flt3 and only minor fractions of CMPs expressing Flt3 (76). Expression of Flt3 fits in with the central role of the cytokine Flt3 Ligand (Flt3L) in steady-state DC development (68), inducing the generation of both pDCs and cDCs from BM precursors (Figure 1.2). Flt3L is a type 1 transmemebrane protein that can be proteolytically cleaved to generate a soluble protein. Both isoforms of Flt3L are biologically active and can induce activation of the Flt3 receptor. Although Flt3L mRNA is ubiquitously expressed in hematopoietic and non-hematopoietic tissues, Flt3L protein has only been found in stromal fibroblasts present in the BM and in T lymphocytes (77).

The cytokine GM-CSF (granulocyte macrophage colony-stimulating factor) can also induce the differentiation of DCs from BM precursors and monocytes (65). Monocytes are phagocytic cells of the myeloid lineage that are located within several organs, such as the blood, BM and spleen (78). Two principal subsets monocyte subsets have been identified in mice that are described as being $\mathrm{Ly}_{6} \mathrm{C}^{\text {high }} \mathrm{CX}_{3} \mathrm{CR}^{\text {low }} \mathrm{CCR} 2^{+} \mathrm{CD} 2 \mathrm{~L}^{+}$( $\mathrm{Ly} 6 \mathrm{C}^{\text {high }}$ ) and Ly6C ${ }^{\text {low }} \mathrm{CX}_{3} \mathrm{CR}^{\text {high }}{ }^{\mathrm{CCR}}{ }^{-} \mathrm{CD} 6 \mathrm{~L}^{-}$(Ly6C ${ }^{\text {low }}$ ) (79), and are proposed to be equivalents of human $\mathrm{CD}_{14}{ }^{+}$and $\mathrm{CD} 16^{+}$monocytes, respectively (80). Ly6 $\mathrm{C}^{\text {high }}$ monocytes are referred to as "inflammatory" monocytes because of their rapid recruitment to sites of experimentally induced inflammation (81) and have been shown to be predisposed to becoming DCs following LPS insult in vivo. 
During infection, monocytes migrate from the blood to the peripheral tissues whereby they have the capacity to differentiate into a number of cell types depending on the soluble mediators that are present within the local environment. In both mice and humans, monocytes differentiate into macrophages in response to M-CSF (macrophage colonystimulating factor) (78) or into cDCs in the presences of GM-CSF (65) (Figure 1.2). GMCSF is produced by tissue stromal cells (82) and by activated T cells, macrophages and endothelial cells upon exposure to antigen or inflammatory cytokines (83). Accordingly, GM-CSF levels in the serum are reportedly low and undetectable within the steady-state, but increases during inflammation (84). GM-CSF regulates its biological activity through activation of the GM-CSF receptor (GM-CSFR) that is expressed on various cell types such as monocytes and CD34 $4^{+}$progenitor cells (83). GM-CSFR is a member of the type I cytokine receptor group and is comprised of an $\alpha$ and $\beta$ subunit (85). The $\alpha$ subunit is responsible for cytokine ligation and is specific for GM-CSF, whereas the $\beta$ subunit is necessary for signal transduction and is also shared with the cytokines IL-3 and IL-5 (83). GM-CSF differs in activity to Flt3L. While Flt3L induces both myeloid-related and lymphoid-related DCs in lymphoid tissues $(74,86)$, GM-CSF only induces the myeloid subtype in vivo (87). 


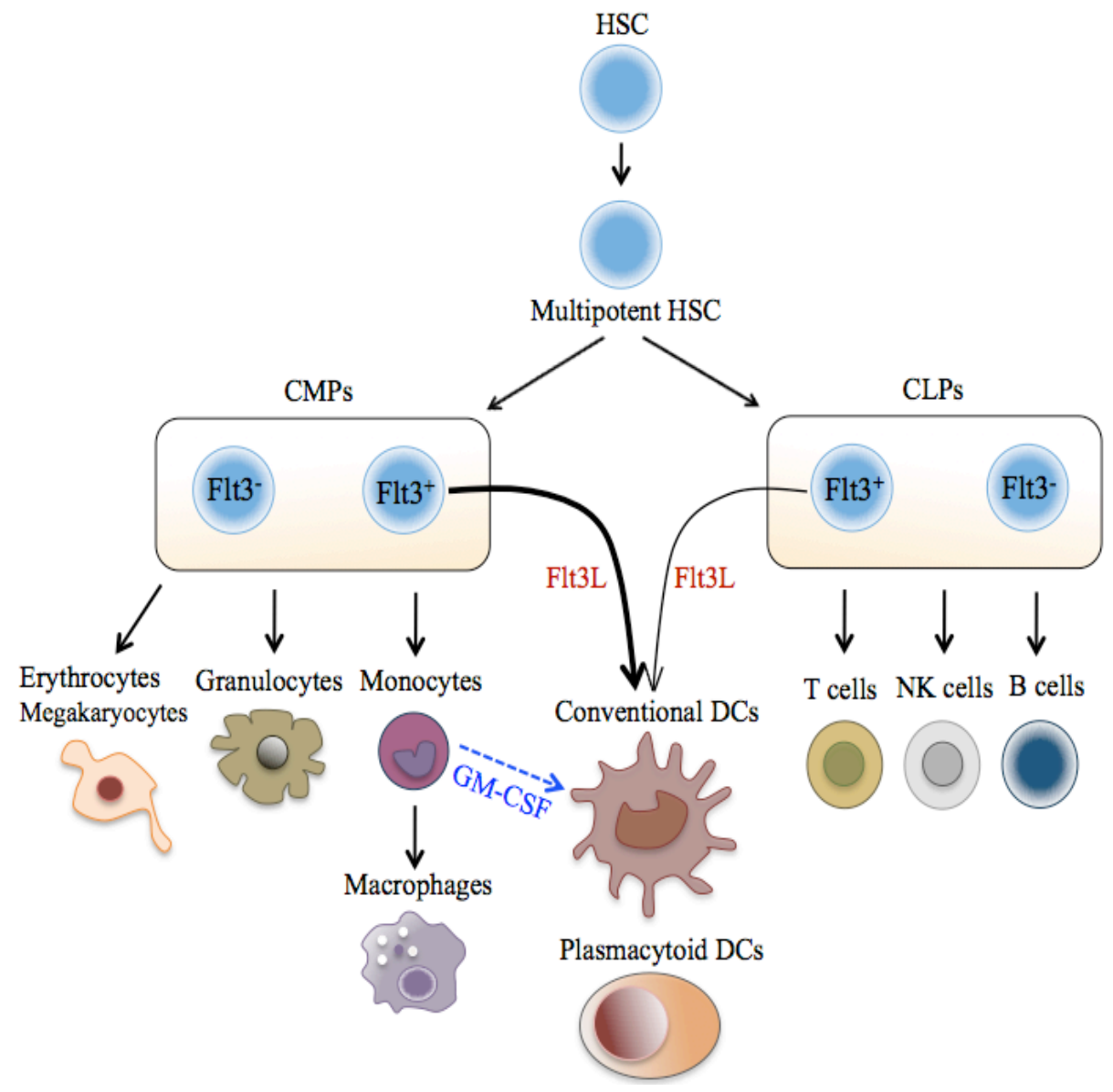

Figure 1.2: DC development from hematopoietic precursors.

Both conventional DCs and plasmacytoid DCs can be generated from the common myeloid progenitors (CMPs) or common lymphoid progenitors (CLPs) expressing Flt3 ${ }^{+}$. Flt3L (red) is essential for the development of steady-state DCs. In the presence of GM-CSF (blue), monocytes can differentiate into cDCs. Myeloid precursors are the main source of DCs in most circumstances (Thick, black curved arrow). Adapted from Naik et al (68) and Wu et al (65).

\subsection{DC subsets}

DCs are a heterogeneous cell population and there are many distinct subsets which differ in location and function within the immune system (88). The two main types of DCs within the immune system are cDCs and pDCs, which are further subdivided based on their location and expression of phenotypic markers. These are discussed as follows. 


\subsubsection{Plasmacytoid DCs}

Human pDCs are indentified as $\mathrm{CD}^{+}{ }^{+} \mathrm{CD} 45 \mathrm{RA}^{+} \mathrm{IL}-3 \mathrm{R} \alpha^{+} \mathrm{ILT}^{+}{ }^{+} \mathrm{ILT} 1^{-} \mathrm{CD} 11 \mathrm{c}^{-}$lineage ${ }^{-}$cells (89) and are phenotypically distinct to mouse pDCs, which are $\mathrm{CD} 11 \mathrm{c}^{\mathrm{int}} \mathrm{CD} 45 \mathrm{RA}^{+} \mathrm{B} 220^{+}$ (65). pDC activation is induced by recognition of viral RNA and DNA, recognised via signalling through receptors TLR7 and TLR9, respectively (90). Following activation, pDCs secrete large amounts of type 1 interferons (IFN), mainly IFN- $\alpha$ and IFN- $\beta$ (91). Through secretion of type 1 IFNs, pDCs inhibit viral replication and enhance the cytotoxicity of immune effector cells such as $\mathrm{NK}$ cells, $\mathrm{CD} 8^{+} \mathrm{T}$ cells and macrophages to eliminate infected cells $(92,93)$. Once activated, pDCs can also present antigen, however, they do not induce $\mathrm{T}$ cell proliferation and effector differentation as efficiently as cDCs. This is thought to be because pDCs do not phagocytose, process and load antigens onto MHC molecules as effectively and express lower levels of MHC II and co-stimulatory molecules (92). Thus, pDCs play an important role in immune responses, particularly to anti-viral immunity.

\subsubsection{Conventional DCs}

Conventional DCs can be divided into those that are migratory or lymphoid tissue-resident. Migratory DCs differ in their tissue of origin. Langerhans cells (LC) are prominent cells within the epidermis and are the model migratory DCs (68). LCs serves as antigensampling sentinels in the peripheral tissues, then migrating to the draining $\mathrm{LN}$ for antigen presentation to $\mathrm{T}$ cells (68). Phenotypically, LCs are langerin ${ }^{+} \mathrm{MHC} \mathrm{II}^{+} \mathrm{CD} 11 \mathrm{c}^{+} \mathrm{CD} 11 \mathrm{~b}^{+}$ EpCAM $^{\text {high }}{ }^{2} 11^{-}$(94). LCs also constitutively express the receptor CD205 (DEC205), a lectin that is implicated in antigen capture and processing (95). Until recently it was generally assumed that the expression of langerin in the skin was strictly confined to LCs in the epidermis. However, it has now become clear that a group of migratory dermal DCs also express langerin $(96,97)$. LCs and dermal langerin ${ }^{+}$DCs are indistinguishable based on the expression levels of MHC II, CD11c and langerin, but can be separated by expression of other markers such as CD103, which is expressed only dermal langerin ${ }^{+}$DCs and not LCs (94). Two subset of dermal langerin ${ }^{+}$DCs have been indentified, each functionally distinct. $\mathrm{CD} 103^{+} \mathrm{CD} 11 \mathrm{~b}^{-}$dermal DCs are proficient at cross-presentation of 
viral antigens to $\mathrm{CD} 8^{+} \mathrm{T}$ cells, whereas $\mathrm{CD} 103^{-} \mathrm{CD} 11 \mathrm{~b}^{+}$are better at priming $\mathrm{CD} 4^{+} \mathrm{T}$ cell responses (98). Cross-presentation refers to the presentation of exogenous antigen to $\mathrm{CD} 8^{+}$ $\mathrm{T}$ cells, and will be further discussed in section 1.7.3.

The spleen is a rich source of lymphoid tissue-resident DCs (65). There are three DC subsets, which have been identified in the spleen of mice that are distinguished by their expression of CD4 and CD8: $\mathrm{CD}^{-} \mathrm{CD}^{+}\left(\mathrm{CD} 8^{+} \mathrm{DCs}\right), \mathrm{CD}^{+} \mathrm{CD} 8^{-}\left(\mathrm{CD} 8^{-} \mathrm{DCs}\right)$ and $\mathrm{CD} 4^{-}$ CD8- (double negative [DN] DCs) (99). Expression of the T cell markers CD4 and CD8 allows for identification of these subsets, however, neither expression have been found to have any functional attributes (88). CD8 on splenic DCs is in the form of an $\alpha \alpha-$ homodimer rather than the $\alpha \beta$-heterodimer that is typical of $\mathrm{T}$ cells (88). Both $\mathrm{CD} 8^{+}$and CD8 ${ }^{-}$DCs differ in location and function within the immune system. In contrast to $\mathrm{CD} 8^{+}$ DCs which are found mainly in T cell areas, CD8 ${ }^{-}$DCs tend to be found in the marginal zones and migrate to $\mathrm{T}$ cell areas upon maturation (100). DC subsets also differ in function, with $\mathrm{CD}^{+}$DCs producing the largest amount of IL-12 after stimulation. Furthermore, $\mathrm{CD}^{+}$DCs have been shown to be the most efficient at cross-presentation of soluble antigen (101), bacteria (102) and viruses (103). The superior cross-presenting ability of $\mathrm{CD}^{+} \mathrm{DCs}$ is not a function of increased antigen uptake (104), but is more likely due to differences in antigen processing. Accordingly, reduced acidification of phagosomes, that is essential for cross-presentation, has been shown to be particularly effective in $\mathrm{CD}^{+} \mathrm{DCs}(105)$. In contrast to $\mathrm{CD} 8^{+} \mathrm{DCs}, \mathrm{CD} 8^{-} \mathrm{DCs}$ appear more efficient at antigen presentation on MHC II molecules to CD4 ${ }^{+} \mathrm{T}$ cells (106).

In addition to differences in function, $\mathrm{CD}^{+}$and $\mathrm{CD} 8^{-} \mathrm{DCs}$ also differ in expression of tolllike receptors (TLR), which recognise microbial products. DCs can express multiple TLRs, thus enabling them to detect a wide range of microbial derived products. All murine splenic DC subsets express TLRs 1, 2, 4, 6, 8, and 9 (107). However, TLR3, which binds to double-stranded RNA is only expressed on $\mathrm{CD}^{+}$DCs (108) and is thus implicated in recognition of viruses. Conversely, $\mathrm{CD}^{-}$DCs do not express TLRs 5 and 7, which recognise bacterial flagellin and single-stranded RNA, respectively $(107,109)$. Overall, distinct TLR expression profiles suggest that DCs specialise in responding to different types of pathogens and highlight the functional diversity of the splenic DCs. 


\subsection{Activation states of DCs}

\subsubsection{Immature DCs}

In the immature state, DCs reside in the peripheral tissues constantly sampling the environment for foreign antigen (110). Immature DCs are phenotypically characterised by low surface expression of major histocompatibility complex class II molecules (MHC II) $(64,66)$ and co-stimulatory molecules such as CD40, CD80 and CD86 (111). Expression of these molecules correlates with T-cell-priming ability, thus immature DCs are weak stimulators of T cell-mediated immunity. Functionally, immature DCs are very efficient at antigen capture and utilise a variety of pathways such as receptor-mediated endocytosis (66) and macropinocytosis (112) to take up different forms of antigen. In receptormediated endocytosis, immature DCs use a range of receptors such as DEC205 (CD205) (95), Fc $\gamma$ receptors type I and II (for uptake of immune complexes or opsonised particles) (113) and scavenger receptors such as CD36 (114). They are also highly phagocytic and can take up bacteria (115) or apoptotic and necrotic cells $(116,117)$. Taken together, immature DCs are specialists in antigen capture, but are weak stimulators of T cellmediated immunity due low expression of MHC and co-stimulatory molecules.

\subsubsection{Mature tolerogenic DCs}

Under the steady-state, immature DCs in the peripheral tissues spontaneously migrate to the draining lymph nodes in the absence of pathogenic stimuli. It is thought that disruption of E-cadherin adhesion between DCs and neighbouring cells induces migration of steadystate DCs (118). During their migration towards the draining LNs, DCs continually capture self-antigens from tissues (119) and become spontaneously matured. Maturation corresponds with the up-regulation of MHC and co-stimulatory molecules, as well as homing receptors, which allows the DC to migrate to the LN (120). However, because these DCs have not been exposed to an inflammatory stimulus, they lack the ability to secrete inflammatory cytokines such as TNF- $\alpha$ or IL-12 (121). Migration to the LN results in antigen presentation to naïve $\mathrm{CD}^{+}$and $\mathrm{CD} 8^{+} \mathrm{T}$ cells, however due to their inability to secrete immuno-stimulatory cytokines (IL-12), these DCs regarded are as quiescent or 
tolerogenic (118). Tolerogenic DCs are able to induce proliferation of naïve T cells, but this does not result in the development of cytokine producing, effector T cells (122). Instead antigen presentation by tolerogenic DCs results in $\mathrm{T}$ cell deletion, or in $\mathrm{T}$ cells becoming unresponsive to further antigen stimulation (123-125). Overall, this process is important in order to maintain peripheral tolerance and is thought to ensure the elimination of auto-reactive $\mathrm{T}$ cells that have escaped thymic deletion (126).

\subsubsection{Mature DCs}

DC maturation and activation is a process which describes the DCs terminal differentiation from poorly immunogenic cells specialized for antigen capture into those with high capacity of T cell stimulation (59). Immature DCs respond to two types of signals that induces their activation and maturation: recognition of pathogen associated molecule patterns (PAMPS) or inflammatory cytokines (127). Various PAMPS are recognized by receptors, such as Toll-like receptors on DCs (128), and inflammatory cytokines such as TNF- $\alpha$ are recognised by the corresponding cytokine receptors. Activation and maturation induces DCs to exert entirely different phenotypic and functional properties, compared to immature DCs. DC maturation results in transiently enhanced uptake of antigen (129) that is followed by an almost complete down-regulation (130). Matured DCs display enhanced capacity of T cell stimulation, caused by the up-regulation of MHC II and co-stimulatory molecules such as CD80, CD86 and CD40 $(131,132)$. CD80 and CD86 are co-stimulatory molecules of the immunoglobulin superfamily (133) and CD40 belongs to the tumour necosis factor receptor superfamily. CD86 is constitutively expressed at low levels and rapidly up-regulated upon DC activation, whereas CD80 expression is inducible and upregulation occurs more slowly. CD80 or CD86 subsequently bind to CD28 and CD40 bind to CD40 ligand (CD40L) that is expressed on activated $\mathrm{T}$ cells, thus inducing $\mathrm{T}$ cell activation (further discussed in section 1.7). Peripheral DCs also change expression of chemokine receptors, and increase expression of CCR7 and CXCR4, thus enabling their migration to secondary lymphoid organs for antigen presentation (134). DC activation also induces secretion of inflammatory cytokines. Depending on the type of PAMPs that induces activation, DCs induce production pro-inflammatory cytokines such IL-6, IL-12, TNF $\alpha$ and IL-23 (135-137). The ability of DCs to produce cytokines such as IL-12, TNF- 
$\alpha$ and IL-6 is thought to be important for the induction of robust T cell responses (50). Within the context of DC-based immunotherapy, studies have demonstrated that DC maturation is crucial for the induction of effective immune responses (138). The "golden standard" used to mature clinical grade DCs consists of cocktails of pro-inflammatory cytokines containing IL-1 $\beta$, IL-6, TNF- $\alpha$ and prostaglandin E2 (139). However, DCs that are exposed to inflammatory cytokines can induce the up-regulation of co-stimulatory molecules, but cannot secrete IL-12 (140). Therefore, DCs matured with cytokine cocktails alone are not as effective at stimulating immune responses as DCs that encounter pathogens in vivo.

\subsection{DC activation through Toll-like receptors}

One critical function of DCs is their ability to recognise foreign pathogens and initiate immune responses. To this end, DCs express several pattern recognition receptors (PPRs), such as Toll-like receptors (TLRs) that can recognise PAMPs (141). TLRs are type 1 transmembrane receptors with a ligand binding domain of leucine-rich repeats and a TollIL-1 receptor (TIR) domain that activates down-stream signalling pathways (142). The binding of PAMPs to the corresponding TLR induces DC activation and an immune response against that particular pathogen. Thus, agonists for TLRs can be used as natural adjuvants to induce DC activation in vitro.

Several TLRs have been characterised to date and can be grouped into those that are on the cell surface or localised intracellularly. The first group of TLRs: TLR1, 2, 4, 5 and 6 are all present on the cell surface (142) and are specialists in the recognition of extracellular bacterial or protozoan compounds. While most TLRs function as homodimers, TLR2 forms heterodimers with TLR1 and TLR6 (143). Accordingly, TLR2/TLR1 or TLR2/TLR6 recognise triacylated or diacylated lipopeptides, respectively (142). TLR4 binds to LPS on Gram-negative bacteria (144) and TLR5 binds to flagellin of motile bacteria (145). The second group of TLRs, TLR3, 7, 8 and 9, all reside intracellularly within the endosomes and facilitate the recognition of viral or microbial nucleic acids (146). TLR7 and 8 bind to single-stranded RNA (ssRNA) while TLR3 binds to double- 
stranded RNA (dsRNA) (146). Conversely, TLR9 binds to unmethylated CpG motifs in DNA (147).

Several synthetic agonists have been developed to induce DC activation in vitro. For example, Pam3Cys (a synthetic lipopeptide) can be used to induce DC activation through TLR2 and Poly I:C (synthetic analogue of dsRNA) can activate DCs through TLR3.

After recognizing their respective PAMPs, TLRs activate signalling pathways that provide specific immunological responses. Different TLRs utilise different adaptors to induce the production of a variety of pro-inflammatory cytokines (148). This will be further discussed in Chapter 3 (section 3.1.1).

\subsection{Antigen presentation to $T$ cells}

There are two major subset of $\mathrm{T}$ cells within the immune system that are distinguished by their surface expression of CD4 or CD8. Both $\mathrm{CD} 4^{+}$and $\mathrm{CD} 8^{+} \mathrm{T}$ cells recognise antigen that have been processed and presented as peptides by DCs on antigen presenting molecules called major histocompatability complex class I or II (MHC I or MHC II). $\mathrm{CD} 4^{+} \mathrm{T}$ cells recognise peptides presented on MHC II and $\mathrm{CD} 8^{+} \mathrm{T}$ cells recognise peptides presented on MHC I. Pathways of antigen processing (commonly referred to as classical presentation pathways) are distinct between the two MHC molecules and are discussed as follows.

\subsubsection{MHC I presentation}

MHC I molecules are expressed on all nucleated cells. These molecules generally present peptides that are only eight to ten amino acids in length, due to conserved hydrogen bonds that close the peptide-binding groove at both ends (149). Classically, peptides presented on MHC I are derived from proteins expressed within the cell (endogenous proteins) (63). Therefore, proteins of viral origin $(150,151)$ or mutated self proteins will be processed and presented. To generate the appropriate peptides, proteins are first degraded by the 
proteosome within the cytosol and then transported into the endoplasmic reticulum (ER) via the Transporter of antigen-processing (TAP)-1 and TAP2 molecules (152). MHC I molecules are synthesised in the ER, where they are assembled with $\beta_{2}$-microglobulin (153). This MHC I- $\beta_{2}$-microglobulin complex then binds to other chaperone proteins including calreticulin and tapasin (154). The binding of the MHC I-chaperone complex to TAP via tapasin allows the loading of peptides onto the MHC I molecule and the subsequent release of the MHC I molecule from the chaperone proteins (155). The peptide-loaded MHC I complex is transported to the cell surface via the Golgi cisternae and present their peptides to $\mathrm{CD} 8^{+} \mathrm{T}$ cells (153).

\subsubsection{MHC II presentation}

Exogenous antigen is classically presented on MHC II molecules, which are only expressed on APCs such as DCs, B cells and macrophages. In contrast to MHC I, MHC II molecules can present longer peptides because their peptide-binding groove is open at both ends (149). Within the MHC II presentation pathway, DCs take up extracellular antigens and deliver it to the lysosomes and late endosomes and whereby it is processed into peptides (156). MHC II molecules are also synthesised within the ER $(157,158)$ and are associated with an invariant chain (li) $(159,160)$. The invariant chain binds to the peptidebinding groove, therefore stabilising the MHC II molecule and preventing binding of peptides or partly folded proteins (161). The MHC II-li complex is transported to the late endosomal compartments, where the li chain is released through proteolytic cleavage (159, 160 ), leaving a short peptide fragment CLIP (class II-associated invariant chain peptide) in the groove of the MHC II molecule (162). The chaperone HLA-DM catalyses the release of the CLIP fragment and the loading of the peptide fragments onto the MHC II molecules $(163,164)$. The peptide-MHC II complex is then exported to the plasma membrane and presented to $\mathrm{CD}^{+} \mathrm{T}$ cells (165).

\subsubsection{Cross-presentation}

The classical pathways of antigen presentation only allows presentation of endogenous antigen on MHC I molecules. However, this does not explain how $\mathrm{CD}^{+} \mathrm{T}$ cell responses 
can be initiated against tumour antigens or viruses that do not infect the APC. In the 1970 's, Bevan was the first to demonstrate that priming of $\mathrm{CD} 8^{+} \mathrm{T}$ cell responses in vivo could also occur after presentation of exogenous antigens by MHC I molecules (166). Bevan initially called this process 'cross-priming' and the term cross-presentation has since been adopted to describe this pathway of antigen presentation. Cross-presentation has been observed in a variety of APCs such as macrophages $(167,168)$ and B cells $(169)$, however DCs have been shown to be the most efficient (170).

The exact mechanisms by which exogenous antigen access the MHC I presentation pathway have not been elucidated and three pathways have been proposed to describe the process of cross-presentation (171). The vacuolar route described cross-presentation in a TAP-independent manner. In this pathway, exogenous antigens do not gain access to the cytosol and are degraded by proteases within endosomal or lysosomal compartments and the resulting peptides bind to $\mathrm{MHC}$ class I molecules that recycle from the cell surface (172, 173). The other two pathways, described cross-presentation in a TAP-dependent manner. The cytosolic route uses the normal MHC I machinery to present exogenous antigens and requires that the antigen is diverted into the cytosol for proteosomal degradation, and subsequent transport into the ER via TAP $(174,175)$. Within the phagosome-ER-fusion route, the phagosome fuses with the ER membrane, therefore allowing proteins required for MHC I loading as well as TAP transporters to enter the phagosome. Antigen is diverted to the cytosol and then degraded locally by proteosomes and then the protesome-derive peptides are transported back into the phagosome via TAP, where it is loaded onto the MHC I molecule $(176,177)$.

\subsection{Induction of $\mathrm{T}$ cell activation by $\mathrm{DCs}$}

Activated and matured DCs initiate or "prime" T cell responses in the secondary lymphoid organs such as the LN and the spleen (178). T cell activation and clonal expansion is induced by signals provided by the interaction of peptide/MHC complexes on the surface of DC and the antigen-specific TCR on T cells. However, full activation of naïve T cells is not only dependent upon antigen presentation but also requires co-stimulatory signals provided by the DC. The co-stimulatory molecules CD80 and CD86, which are highly 
expressed on activated DCs binds to CD28 on the T cells $(179,180)$. The engagement of CD28 on the T cell by CD80 or CD86 drives the cell cycle progression of T cells (181), promotes $\mathrm{T}$ cell survival by enhancing the expression of anti-apoptotic protein $\mathrm{Bcl}-\mathrm{X}_{\mathrm{L}}$ (182) and lowers the antigen stimulation threshold required to induce cellular division of T cells (183). Activated DCs also express other co-stimulatory molecules such as CD40, which binds to CD40L expressed on activated T cells (184). Engagement of CD40 and CD40L activates DCs, leading to IL-12 production (185) and increased expression of costimulatory molecules on the DC (186). Effectively, IL-12 production from DCs promotes $\mathrm{T}$ cell differentiation while the increased expression of co-stimulatory molecules on DCs enhances the level of co-stimulation available to $\mathrm{T}$ cells.

$\mathrm{T}$ cell activation also results in expression of early activation markers such as CD25 and CD69 (187) on T cells. Signalling through CD69 leads to calcium influx, cytokine secretion and CD25 up-regulation, which ultimately induce T cell proliferation (188). Activated T cells also acquire tissue homing receptors (189), allowing their migration from the LN into the periphery where they carry out their effector functions.

\subsection{1 $\mathrm{CD4}^{+} \mathrm{T}$ cell activation and effector function}

Although $\mathrm{CD}^{+} \mathrm{T}$ cells are effector cell in their own right, they are also called $\mathrm{T}$ helper (Th) cells because they play an important role in enhancing the function of other immune cell-types. Activation of naïve $\mathrm{CD}^{+} \mathrm{T}$ cells induces their differentiation into at least two functionally distinct subsets (Th1 and Th2) (190), which is dependent upon the cytokine milieu at the time of activation. Th1 cells produce high levels of their signature cytokine IFN- $\gamma$, and their developmental pathway is driven by the cytokine IL-12 (191). IFN- $\gamma$ have important roles in the immune response, such as inducing the activation and recruitment of other cell types (i.e. macrophages) and the priming of $\mathrm{CD}^{+} \mathrm{T}$ cell responses (191). Alternatively, Th2 cells are characterised by production of IL-4, IL-5 and IL-13 (190) and their differentiation is driven by a cytokine milieu rich in IL-4 (192) and IL-6 (193). Each Th subset mediate distinct immunologic effector functions: Th1 cells are associated with responses to intracellular pathogens such as viruses, while Th2 mediate protection from extracellular pathogens such as helminths (194). $\mathrm{CD}^{+} \mathrm{T}$ activation are critical for 
effective anit-tumour immune responses and their specific action will be discussed in Chapter 4 (section 4.1.1).

\subsection{2 $\mathrm{CD8}^{+} \mathrm{T}$ cell activation and effector function}

The main role of $\mathrm{CD}^{+} \mathrm{T}$ cells is to kill infected or malignant cells and DCs play a central role in the priming of $\mathrm{CD}^{+} \mathrm{T}$ cell responses (195). Upon encounter of DCs presenting cognate antigen in the draining LN, antigenic stimulation via engagement of the TCR and peptide/MHC I complex, and co-stimulation induces $\mathrm{CD}^{+} \mathrm{T}$ cell activation. $\mathrm{CD}^{+} \mathrm{T}$ cells thus undergo clonal expansion and differentiate into cytotoxic T lymphocytes (CTL). CTLs also express death receptor ligands and cytotoxic granules, all of which contribute to their ability of killing infected cells (196). The main pathway of killing is exocytosis of granules containing perforin or granzymes. Perforin is a pore-forming protein, which is required to enable granzymes to induce apoptosis of target cells (197). Perforin-mediated immune responses have been shown to be important for mounting effective anti-tumour immunity (198). The exact mechanisms of perforin function are unclear and two models have been proposed. It was thought that perforin directly formed pores in the target cell membrane to allow entrance of granzymes into the cells, or that perforin disrupts targetcell endosomes that contain granzymes (199). Granzymes A and B are responsible for DNA fragmentation in target cells, with Granzyme B being the principal apoptotic molecule in CTLs (200). Another mechanism of target cell lysis used by CTLs is via the death receptor pathway. Expression of death receptors such as Fas (CD95), TNFR-1 and TRAIL receptor (TRAIL-R) targets cells for destruction by activated $\mathrm{CD} 8^{+} \mathrm{T}$ cells expressing the corresponding ligand (201). Fas is the best characterized death receptor that is expressed on many cell types (202) and binds to FasL that is expressed and up-regulated on activated $\mathrm{CD}^{+} \mathrm{T}$ cells upon TCR stimulation (203). Engagement of Fas with FasL allows for target cell killing by caspase-mediated apoptosis (204).

Other effector functions of CTLs include secretion of the cytokines TNF- $\alpha$ and IFN- $\gamma$. IL12 (secreted by activated DCs) stimulates CTLs to produce IFN- $\gamma(205)$. TNF- $\alpha$ can bind to its receptor, TNFR, which is present on the cell surface of target cells to induce programmed cell death (206). The presence of IFN- $\gamma$ has been shown to inhibit tumour 
angiogensis (207) and can also induce expression of MHC I on infected cells, thus increasing their chance of recognition for cytotoxic attack (208).

During the course of the immune response, activation of naïve $\mathrm{CD} 8^{+} \mathrm{T}$ cells not only induces their differentiation into effector CTLs, but also of memory $\mathrm{CD} 8^{+} \mathrm{T}$ cells. Memory $\mathrm{T}$ cells persist in a resting state after infection as long-lived and antigen experienced cells that can confer protection upon a secondary challenge (209).

\subsection{The role of GM-CSF and IL-4 in generating DCs in vitro}

\subsubsection{GM-CSF and IL-4 are commonly used for generating monocyte- derived DCs in clinical trials}

Monocyte-derived DCs (Mo-DC), are commonly used in studies of human DC biology, and for cancer immunotherapy $(139,210)$. Their widespread use in most vaccination studies is due to the relative ease with which large quantities can be obtained. Typically, $100-150 \times 10^{6}$ monocytes can be collected in one leukaphresis, which has the advantage over selection of $\mathrm{CD}^{+} 4^{+}$progenitor cells that often requires repeated leukaphresis (50). The type of DC that is generated in vitro from monocyte differentiation depends on the cytokine cocktail that is used. For instance, the culture of monocytes with the cytokines GMCSF/IL-4 generates immature DCs devoid of LCs (211). In contrast, cytokine cocktails such as GMCSF/IL-15, induce monocyte differentiation into immature DCs with features like LCs (212). GMCSF/IL-4 represents the most extensively utilised cytokine combination for generating DCs from monocytes $(83,213)$. While GM-CSF appears to be required for monocyte survival and differentiation, IL-4 inhibits the development of macrophages (83). The culture of murine BM cells with GMCSF/IL-4 has been shown to generate a population of immature DCs that are $\mathrm{CD} 24^{\text {low }} \mathrm{CD} 11 \mathrm{~b}$ high and often referred to as "myeloid" DCs because of their CD11b $\mathrm{b}^{\text {high }}$ phenotype (214). Similarly, monocytes

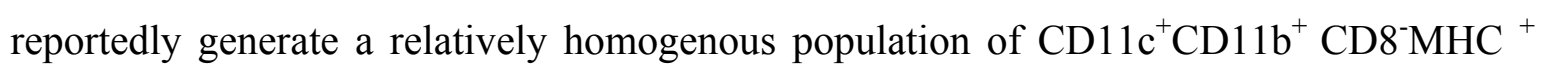
myeloid DCs in GM-CSF cultures (78).

Several studies of DC vaccination using Mo-DCs have been performed in patients with 
melanoma (50), for which many immunologically relevant antigens have been defined (215). A good overview of DC-vaccination trials using matured and antigen loaded MoDCs generated in GMCSF/IL-4 cultures, was compiled by Lesterhius et al (50). Many of these Mo-DCs were induced to mature by exposure to the inflammatory cytokine TNF- $\alpha$ alone or with one or more of the following cytokines: IL-1 $\beta$, IL-6 or prostaglandin-E2. Overall, it was shown that Mo-DCs have shown some success at inducing anti-tumour immune responses (50), such as the expansion of tumour-specific CTLs (216) or tumourspecific Th1 cells (217). However, the objective clinical outcomes were variable, with complete, partial or mixed responses seen throughout. Despite the extensive use of GMCSF/IL-4 in generating Mo-DCs, the question was raised as to whether this cytokine combination reflects the natural pathway of DC generation in vivo (83). Evidence now suggests that Mo-DCs generated from GMCSF/IL-4 cultures are representative of DCs that arise in vivo during inflammation. These findings are discussed in the following section.

\subsubsection{GM-CSF induces the development of inflammatory Mo-DCs}

Studies using knockout mice first supported the idea that GM-CSF is not required for maintenance of steady-state DCs. This was shown whereby GM-CSF or GMCSFR knockout mice had only a small (20-24\%) reduction of DCs in their spleen and thymus, compared to wild-type controls (218). Further studies began to provide more correlative data for the role of GM-CSF in inflammatory DC development. The first clear hint was derived from studies in mice following infection with the gram-positive bacteria, Listeria monocytogenes (78).

Within a mouse model, intravenous inoculation with L. monocytogenes, results in their rapid clearance from the bloodstream and predominant infection within the spleen and liver. L. monocytogenes subsequently proliferates in vivo for 2 or 3 days and is cleared upon induction of antigen-specific $\mathrm{CD}^{+} \mathrm{T}$ cell response (80). In 1988, Cheers et al were able to show that infection with $L$ monocytogenes, induced higher serum levels of GMCSF in mice (79). Accordingly, Serbina et al later showed that a novel DC subtype emerges in the spleen of mice 1-2 days post infection with L. monocytogenes, which produce the inflammatory mediators iNOS and TNF- $\alpha$. Production of reactive nitrogen 
intermediates by iNOS is one of the major mechanisms for killing of L. monocytogenes in vivo (80). These iNOS/TNF- $\alpha$ producing DCs (Tip-DCs) were CD11 $\mathrm{c}^{\text {int }}$ CD11b ${ }^{\text {high }}$ MAC$3^{+}$and phenotypically distinct from splenic steady-state DCs which are CD11 $\mathrm{c}^{\text {high }} \mathrm{CD} 11 \mathrm{~b}^{\text {low }} \mathrm{MAC}-3^{-}$(80). It was also found that Tip-DCs were absent from the spleen of CCR2 knockout mice, which lack the chemokine receptor implicated in macrophage and DC migration. Given the finding by other authors that the Ly6 $\mathrm{C}^{\mathrm{hi}}$ monocyte subset selectively expresses CCR2, this lead on to the presumption that Ly6C ${ }^{\text {hi }}$ monocytes were the contributing precursor for differentiation of inflammatory Tip-DCs during infection (78). Collectively, these findings by Serbina et al and Cheers et al provided the first indication of the in vivo equivalent of GM-CSF-driven monocyte differentiation.

Subsequent experiments performed by Naik et al directly provided evidence for the role of GM-CSF in inducing the differentiation of Ly6C ${ }^{\text {hi }}$ monocytes into Tip-DCs in vivo (219). Within their studies, Ly6 $\mathrm{C}^{\text {hi }}$ monocytes were transferred intravenously into mice under conditions of GM-CSF-dependent inflammation. This was established by priming mice with methylated bovine serum albumin (mBSA) in complete Freund's adjuvant (CFA), followed by induction of inflammation with mBSA 1 day later. Naik et al were able to show that Ly6C ${ }^{\text {hi }}$ monocytes developed into CD11c ${ }^{+} \mathrm{MHC} \mathrm{II}^{-} \mathrm{Mac}-3^{+} \mathrm{DCs}$ in the spleen that produced iNOS and TNF- $\alpha$, thus providing formal proof of the correlation between GM-CSF and development of inflammatory DCs (219).

In light of the above evidence, Xu et al (214) were able to show that the BM derived DCs generated in vitro from GMCSF/IL-4 shared characteristics of inflammatory Tip-DCs. Firstly, GMCSF/IL-4 DCs were found to express the markers CD11 $\mathrm{c}^{+} \mathrm{MHC} \mathrm{II}^{+} \mathrm{MAC}-3^{+}$ and produced TNF- $\alpha$ and iNOS in response to stimulation with LPS. Moreover, it was shown that $\mathrm{CD} 11 \mathrm{~b}^{\text {high }} \mathrm{Ly} 6 \mathrm{C}^{\text {high }}$ monocytes isolated from murine $\mathrm{BM}$ developed into CD11 $\mathrm{c}^{+} \mathrm{MHC} \mathrm{II}^{+} \mathrm{DCs}$ when cultured with GMCSF/IL-4. These CD11 $\mathrm{c}^{+} \mathrm{MHC} \mathrm{II}^{+} \mathrm{DCs}$ also expressed the marker MAC- $3^{+}$and secreted iNOS following LPS stimulation (214). Overall, these results clearly demonstrate the developmental linkage between inflammatory conditions and GMCSF/IL-4 DCs. 


\subsection{Flt3L is crucial for normal hematopoiesis and DC development in vivo}

Flt3L is a hematopoietic cytokine that binds to the receptor Flt3, which is a tyrosine kinase receptor highly expressed on HSCs (refer to section 1.3.2). Hannum et al (220) and Lyman et al (221) were the first to clone Flt3L in 1993-1994, thus enabling investigation of its actions in vivo. Studies in mice with targeted gene disruption in Flt3L have established the importance of this cytokine in maintaining normal hematopoiesis and DC development. Specifically, McKenna et al showed that mice with genetic deletion of Flt3L displayed a reduction in leukocyte numbers in the peripheral blood, LN and spleen (222). In particular, targeted deletion of Flt3L induced about a 5-fold reduction in NK cells and a 4 to14-fold reduction of $\mathrm{CD}^{-}$and $\mathrm{CD}^{+} \mathrm{cDCs}$ in the spleen (222). Further to the findings of McKenna et al, other authors have shown that treatment with Flt3 kinase inhibitors also leads to a 10-fold reduction of pDCs in the spleen and LN of mice (223). Moreover, mice with constitutively active Flt3 signalling were prone to development of leukaemia (224). Together these data provide strong evidence that Flt3L is necessary for the in vivo generation of DCs in the steady state.

\subsection{Flt3L BM cultures generate DCs that are equivalent to steady- state DCs in vivo}

Naik et al were the first to provide correlative data showing that the culture of murine BM cells with Flt3L generated three DC subsets that are close equivalents of the steady-state splenic pDCs, $\mathrm{CD}^{+} \mathrm{cDC}$ and $\mathrm{CD}^{-} \mathrm{cDC}$ subtypes (225). This was demonstrated by several experiments, firstly analysing the surface phenotype of DCs generated from Flt3L cultures (Flt3L-DCs) and comparing it to splenic DC subsets. Similar to splenic DCs, Flt3L-DCs contained two primary subsets that consisted of $\mathrm{CD} 11 \mathrm{c}^{+} \mathrm{CD}_{4} \mathrm{RA} \mathrm{R}^{-} \mathrm{cDCs}$ and

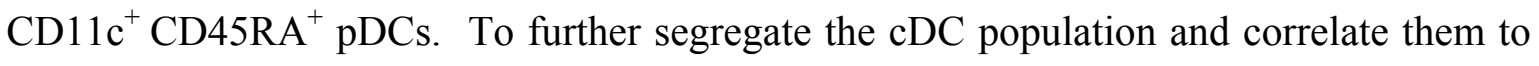
splenic $\mathrm{CD}^{+} \mathrm{cDC}$ and $\mathrm{CD} 8^{-} \mathrm{cDCs}$, the markers CD24 and SIRP- $\alpha$ were assessed among

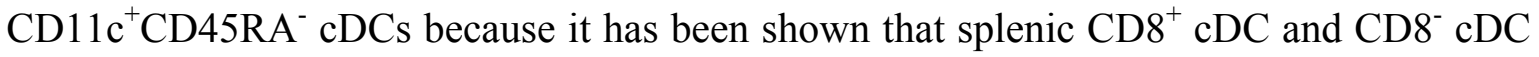
selectively express CD24 and SIRP- $\alpha$, respectively. Indeed, these markers allowed the indentification of two cDCs subsets that were CD24 $4^{\text {high }} \mathrm{SIRP}-\alpha^{\text {low }} \mathrm{CD} 11 b^{\text {low }}\left(\mathrm{CD} 8^{+} \mathrm{cDC}\right.$ equivalents) and $\mathrm{CD} 24^{\text {low }} \mathrm{SIRP}-\alpha^{\text {high }} \mathrm{CD} 11 \mathrm{~b}^{\text {high }}$ (CD8- $\mathrm{cDC}$ equivalents). Therefore, it was 
shown that Flt3L-DCs and splenic DCs do exhibit similarities in phenotype. Furthermore, correlative data were also gathered by analysis of transcription factors, which vary in their expression among the splenic DC subsets: $\mathrm{CD} 8^{+} \mathrm{cDC}$ mainly express IRF-8, $\mathrm{CD}^{-} \mathrm{cDC}$ predominantly express IRF-4, whereas pDCs express both. In line with splenic DCs, Flt3L-DC subsets also shared the same expression patterns of IRF's. In addition, Naik et al showed an almost identical pattern of TLR mRNA expression and cytokine secretion patterns between Flt3L-DC and splenic DC subsets. Taken together, this study was able to demonstrate that Flt3L cultures give rise to DCs displaying phenotypic and functional similarities to that of steady-state splenic DCs (225).

In addition to demonstrating that GMCSF/IL-4 DCs correspond to inflammatory Tip-DCs (see section 1.9.2), $\mathrm{Xu}$ et al also directly compared the phenotype and functional characteristics of GMCSF/IL-4 and Flt3L-DCs (214). As previously mentioned, murine $\mathrm{BM}$ cells cultured in GMCSF/IL4 generate DCs that express the markers CD11c ${ }^{+} \mathrm{MHC}$ $\mathrm{II}^{+} \mathrm{MAC}-3^{+}$. In agreement with Naik et al (225), the culture of murine BM cells generated DCs that consisted of $\mathrm{CD} 11 \mathrm{c}^{+} \mathrm{B} 220^{+} \mathrm{pDCs}$ and $\mathrm{CD} 11 \mathrm{c}^{+} \mathrm{B} 220^{-} \mathrm{cDCs}$, which could be subdivided into those that were $\mathrm{CD} 244^{\text {high }} \mathrm{CD} 11 \mathrm{~b}^{\text {low }}$ (CD8 $8^{+}$equivalent) and $\mathrm{CD} 24^{\text {low }} \mathrm{CD} 11 \mathrm{~b}^{\text {high }}$ (CD8- equivalent). In contrast to GMCSF/IL-4 DCs, Flt3L-DCs did not express MAC-3 and were incapable of producing iNOS following LPS stimulation. Furthermore, unlike GMCSF/IL-4, Flt3L could not give rise to CD11 ${ }^{+} \mathrm{MHC} \mathrm{II}^{+}$DCs from CD11b ${ }^{\text {high }}$ Ly6 $\mathrm{C}^{\text {high }}$ monocytes.

In summary, Flt3L-DCs are phenotypically and functionally distinct from GMCSF/IL-4DCs. While GMCSF/IL-4-DCs correspond to monocyte-derived DCs that arise in vivo during inflammation, Flt3L-DCs represent the 3 main subsets of DCs found in lymphoid organs under steady-state conditions. 


\subsection{Objectives of this study}

The general introduction has summarised the roles of DCs within the immune system and their potential use in cancer immunotherapy. As previously discussed, the majority of clinical applications so far have relied on the in vitro generation of DCs from blood monocytes with the cytokines GMCSF/IL-4. However, compared to DCs generated with Flt3L, GMCSF/IL-4 DCs do not reflect the DC subsets found under the steady-state conditions, but instead are similar to DCs generated in vivo during inflammation. Given the differences between the two culture systems, this thesis aimed to assess the suitability of Flt3L-DCs for cancer immunotherapy.

The objectives of this study were:

Aim 1: $\quad$ To characterise the phenotype of Flt3L-DCs and determine their response to stimulation with various TLR agonists.

Aim 2: $\quad$ To investigate the functional properties of Flt3L-DCs.

Aim 3: $\quad$ To determine whether Flt3L-DCs can activate tumour-specific $\mathrm{CD}^{+}{ }^{+}$cells in vivo and induce tumour rejection in mice. 
Chapter 2.

Materials and method 


\subsection{Materials}

\subsubsection{Labware}

\begin{tabular}{|c|c|}
\hline $\begin{array}{l}\text { Product } \\
\end{array}$ & Source \\
\hline $\begin{array}{l}\text { Acroside }^{\mathrm{TM}} 32 \mathrm{~mm} \text { syringe filters with } \\
0.2 \mu \mathrm{m} \text { Supor }{ }^{\circledast} \text { membrane }\end{array}$ & Pall Life Sciences, Cornwall, U.K. \\
\hline $\begin{array}{l}\text { Axygen Sterile pipette tips }(1000,200 \text { and } \\
\text { 10uL) }\end{array}$ & \multirow[t]{2}{*}{ Axygen Scientific Inc, Union city, USA } \\
\hline Axygen Micro Tubes & \\
\hline $\begin{array}{l}\text { BD Falcon }{ }^{\circledR} \text { polystyrene sterile multiwell } \\
\text { tissue culture plates: } 6 \text { well, } 24 \text { well and } \\
\text { Microtest }^{\mathrm{TM}} \text { U-bottom } 96 \text { well plates }\end{array}$ & \multirow[t]{9}{*}{$\begin{array}{l}\text { Becton Dickson (BD) Biosciences, CA, } \\
\text { USA }\end{array}$} \\
\hline $\begin{array}{l}\text { BD Falcon }{ }^{\circledR} \text { polystyrene tissue culture } \\
\text { dishes } 100 \times 20 \mathrm{~mm}\end{array}$ & \\
\hline $\begin{array}{l}\text { BD Falcon }{ }^{\circledR} \text { polystyrene tissue culture } \\
\text { flasks: } 200 \mathrm{~mL} \text { and } 600 \mathrm{~mL}\end{array}$ & \\
\hline $\begin{array}{l}\text { BD Falcon }{ }^{\circledR} \text { polystyrene sterile } 5 \mathrm{~mL} \text { round } \\
\text { bottom tubes }\end{array}$ & \\
\hline $\begin{array}{l}\text { BD Falcon }{ }^{\circledR} \text { polystyrene sterile serological } \\
\text { pipettes: } 5 \mathrm{~mL}, 10 \mathrm{~mL} \& 25 \mathrm{~mL}\end{array}$ & \\
\hline $\begin{array}{l}\text { BD } 1 \mathrm{~mL} \text { tuberculin syringes \& BD } 10 \mathrm{~mL} \\
\text { syringes }\end{array}$ & \\
\hline $\begin{array}{l}\text { Precision } \text { Glide }^{\mathrm{TM}} \text { needles: } 18,20,25 \& \\
27.5 \text { gauge }(\mathrm{G})\end{array}$ & \\
\hline $\begin{array}{l}\text { Ultra-Fine }{ }^{\mathrm{TM}} \text { needle insulin syringes }(29 \mathrm{G}) \text { : } \\
0.3 \mathrm{ml}\end{array}$ & \\
\hline $\begin{array}{l}\text { BD Falcon }{ }^{\circledR} \text { nylon cell strainers }(40 \& \\
70 \mu \mathrm{m})\end{array}$ & \\
\hline Cryo's ${ }^{\mathrm{TM}}$ sterile cryotubes $(2 \mathrm{~mL})$ & Greiner Bio-one, Frickenhausen, Germany \\
\hline Cover Slips $(22 \times 22 \mathrm{~mm})$ No. 1 thickness & BioLab Ltd, Auckland, NZ \\
\hline $30 \mu \mathrm{m}$ MACS ${ }^{\circledR}$ pre-separation filters & Miltenyi Biotech GmbH, Germany \\
\hline
\end{tabular}




\begin{tabular}{|l|l|}
\hline Millipore MX-plates & $\begin{array}{l}\text { Millipore Corporation, Billercia, MA, } \\
\text { USA }\end{array}$ \\
\hline Nylone gauze $(70 \mu \mathrm{m})$ & NZ Filter Specialists Ltd, Auckland, NZ \\
\hline Superfrost ${ }^{\circledR}$ Plus microscope slides & BioLab Ltd, Auckland, NZ \\
\hline Shandon filter cards & Thermo Corporation, USA \\
\hline Titertube $^{\mathrm{TM}}$ Microtubes $0.5 \mathrm{~mL}$ & Bio-Rad Laboratories, Hercules, CA, USA \\
\hline
\end{tabular}

\subsubsection{Reagents and materials}

\section{2-Deoxy-D-Glucose (2-DG)}

2-Deoxy-D-Glucose (MW 0.164) in powdered form was purchased from Sigma (St. Louis, Missouri, USA) and stored at room temperature. When used, 2-DG was dissolved in cIMDM and sterilised through a $0.2 \mu \mathrm{m}$ syringe filter.

\section{2 mercaptoethanol (2 ME)}

$2 \mathrm{ME}$ was purchased from Sigma (St. Louis, Missouri, USA) as a $55 \mathrm{mM}$ solution in PBS and stored at $4^{\circ} \mathrm{C}$.

\section{Acetone}

Analytical grade acetone was purchased from Scharlau Chemi (Barcelona, Spain) and stored at room temperature until used.

\section{Ammonium Chloride Tris (ACT) Lysis Buffer}

ACT buffer was prepared by mixing 9 parts of $0.16 \mathrm{M} \mathrm{NH}_{4} \mathrm{Cl}, \mathrm{pH} 7.4$ (Sigma, St. Louis, Missouri, USA) and 1 part $0.17 \mathrm{M}$ Tris-HCl, pH 7.65 (Merck, Darmstadt, Germany) to give a final concentration of $0.144 \mathrm{M} \mathrm{NH}_{4} \mathrm{Cl}$ and $0.017 \mathrm{M}$ Tris- $\mathrm{HCl}$.

\section{BioMag $®$ Goat anti-Rat IgG Beads}

BioMag ${ }^{\circledR}$ beads were purchased in suspension containing sodium azide and stored at $4{ }^{\circ} \mathrm{C}$ (Bang Laboratories, Inc, USA). 


\section{Bovine Serum Albumin (BSA)}

BSA with low endotoxin levels was purchased from ICPbio Ltd. (Henderson, Auckland, $\mathrm{NZ}$ ) in powder form and stored at $4^{\circ} \mathrm{C}$. BSA was weighed out at $0.5 \mathrm{~g}$ and was added directly to $500 \mathrm{~mL}$ of PBS to give a final concentration $0.1 \%$.

\section{Brefeldin A}

Brefeldin A was purchased from eBioscience (San Diego, CA, USA) at 1000x concentration and stored at $4{ }^{\circ} \mathrm{C}$. When used, Brefeldin A was diluted to a $1 \mathrm{x}$ concentration in IMDM.

\section{DNase I}

DNase I was purchased from Roche (Mannheim, Germany). The lyophilised powder was dissolved to a concentration of $10 \mathrm{mg} / \mathrm{mL}$ in IMDM and stored at $-20^{\circ} \mathrm{C}$.

\section{Ethanol (EtOH)}

Molecular grade 100\% EtOH was purchased from Carlo Erba Reagents (Milan, Italy) and stored at room temperature until used.

\section{Ethylendiaminetetraacetic Acid (EDTA)}

EDTA (Sigma, St. Louis, Missouri, USA) was purchased in powder form and dissolved in $\mathrm{dH}_{2} \mathrm{O}$ to give a stock concentration of $0.5 \mathrm{M}$ and stored at room temperature until used.

\section{Foetal Bovine Serum (FBS)}

FBS was purchased from GIBCO (Invitrogen, Auckland, NZ) and was screened for mycoplasma and virus, which contained $27 \mathrm{EU} / \mathrm{mL}$. FBS was stored in $25 \mathrm{~mL}$ aliquots at $20^{\circ} \mathrm{C}$. After thawing, aliquots were stored at $4^{\circ} \mathrm{C}$ for a maximum of 2 weeks.

\section{Geneticin $®(G 418)$}

The antibiotic Geneticin ${ }^{\circledR}$ was purchased from GIBCO (Invitrogen, Auckland, NZ) and stored in aliquots at $-20^{\circ} \mathrm{C}$. After thawing, aliquots were stored at $4{ }^{\circ} \mathrm{C}$ up to a period of 4 weeks before discarding. 


\section{Iscoves Modified Dulbeco's Medium (IMDM)}

Iscoves Modified Dulbeco's Medium supplemented with GlutaMAX ${ }^{\mathrm{TM}}$, 25mM HEPES buffer and $3.024 \mathrm{mg} / \mathrm{L} \mathrm{NaHCO}_{3}$ was purchased from GIBCO (Invitrogen, Auckland, NZ) and stored at $4{ }^{\circ} \mathrm{C}$.

\section{Complete Iscoves Modified Dulbeco's Medium (cIMDM)}

IMDM was supplemented with 100U/mL Penicillin-streptomycin (GIBCO, Invitrogen,

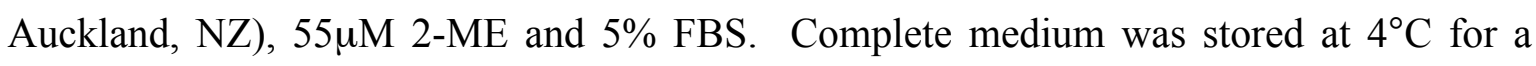
maximum of 3 weeks.

\section{Magnetic Separation (MACS) beads}

Anti-CD8 $\alpha$, anti-CD4 and anti-biotin MACS Microbeads were purchased from Miltenyi Biotec $\mathrm{GmbH}$ (Bergisch Gladbach, Germany) and stored at $4^{\circ} \mathrm{C}$ until used.

\section{Methanol}

Analytical grade methanol was purchased from Scharlau Chemi (Barcelona, Spain) and stored at room temperature.

\section{Penicillin-Streptomycin}

Penicillin-Streptomycin was purchased in liquid form from GIBCO (Invitrogen, Auckland, $\mathrm{NZ}$ ) and stored as single use aliquots at $-20^{\circ} \mathrm{C}$ until used.

\section{Phosphate Buffered Saline (PBS)}

$\mathrm{CaCl}_{2}$ and $\mathrm{MgCl}_{2}$ free PBS was purchased from GIBCO (Invitrogen, Auckland, NZ) and stored at room temperature. When in use, PBS was stored at $4{ }^{\circ} \mathrm{C}$.

\section{Sodium Azide $\left(\mathrm{NaN}_{3}\right)$}

$\mathrm{NaN}_{3}$ (Sigma, St. Louis, Missouri, USA) was purchased in powder form and dissolved in $\mathrm{dH}_{2} \mathrm{O}$ to a stock concentration of $5 \%$. The solution was stored at room temperature until used. 


\section{Tritiated thymidine ([ ${ }^{3}$ H]-thymidine)}

6-Methyl-3H thymidine $(5 \mathrm{mCi})$ with a specific activity of $5 \mathrm{mCi} / \mathrm{mmol}$ was purchased from Amersham Biosciences (Little Chalfont, UK). The stock was diluted in IMDM to a working solution of $20 \mu \mathrm{Ci} / \mathrm{mL}$ and stored at $4^{\circ} \mathrm{C}$.

\section{Trypsin/EDTA}

Trypsin/EDTA solution containing 0.25\% Trypsin and 1mM EDTA in Hank's Balanced Salt Solution was purchased from GIBCO (Invitrogen, Auckland, NZ) and aliquots were stored at $4^{\circ} \mathrm{C}$.

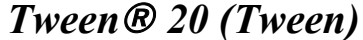

Tween was purchased from Sigma (St. Louis, Missouri, USA) and stored at room temperature until used.

\subsubsection{Cytokines and growth factors}

\section{Granulocyte-colony stimulating factor (GM-CSF)}

Recombinant murine GM-CSF was produced using stationary phase cultures of the murine X63 cell line (226), modified to secrete the full-length murine GM-CSF protein. The modified murine X63 cell line was kindly provided by Dr Antonius Rolink (Basel Institute for Immunology, Basel, Switzerland).

\section{Interleukin $4(I L-4)$}

Recombinant murine IL-4 was produced using stationary phase cultures of CHO cell lines, modified to secrete the full-length murine IL-4 protein. The modified murine $\mathrm{CHO}$ cell line was kindly provided by Dr Antonius Rolink (Basel Institute for Immunology, Basel, Switzerland). 


\section{Fms-like tyrosine kinase ligand (Flt3L)}

Recombinant murine Flt3L was produced using stationary phase cultures of a Chinese Hamster Ovary cell line (CHO) modified to secrete murine Flt3L (227). The modified CHO cell line was kindly provided by Prof Nic Nicola (WEHI, Melbourne, Australia).

Cytokines were collected by growing cell lines in cIMDM in culture flasks. The culture supernatants containing the cytokines were then harvested and filtered through a $0.2 \mu \mathrm{m}$ serum filter. The cytokines were then titrated using BM-DC cultures (GMCSF/IL-4 and Flt3L respectively) to select an optimal concentration for use. For Flt3L, the optimal concentration was determined by those that provided good cell recovery and a clear separation of phenotypes. Flt3L were commonly used at a concentration of $2 \%$ or $4 \%$ throughout this thesis. Optimal concentrations for GMCSF/IL-4 were determined by those that generated DCs with an immature phenotype being less than $70 \%$.

Aliquots of the cytokines described above were stored at $-20^{\circ} \mathrm{C}$ and in use aliquots were stored for up to 2 weeks at $4^{\circ} \mathrm{C}$.

\subsubsection{TLR agonists}

\section{CpG Oligodeoxynucleotide 1668 (CpG)}

Transfection grade CpG ODN 1668 (sequence: 5'-tccatgacgttcctgatgct-3') was purchased as lyophilised powder from GeneWorks (Thebatron, SA, Australia) and stored at $4^{\circ} \mathrm{C}$. To create a working stock, $\mathrm{CpG}$ was dissolved in sterile $\mathrm{PBS}$ to give concentration of $3.2 \mathrm{mg} / \mathrm{mL}$ and aliquots were stored at $-20^{\circ} \mathrm{C}$. In use aliquots were stored at $4{ }^{\circ} \mathrm{C}$ for a maximum of 3 weeks.

\section{Lipopolysaccharide (LPS)}

LPS from Escherichia coli, serotype 0.111:B4, was purchased as lyophilised powder from Sigma (St. Louis, Missouri, USA) and dissolved in sterile IMDM at a stock concentration of $1 \mathrm{mg} / \mathrm{mL}$ and stored at $4^{\circ} \mathrm{C}$. 


\section{$\mathrm{Pam}_{3} \mathrm{Cys}-\mathrm{SKKKK} \mathrm{x} 3 \mathrm{HCl}$ (Pam3Cys)}

Pam3Cys, a synthetic lipopetide based on the structure of bacterial lipoprotein was purchased as a lyophilised powder (EMC microcollections, GmbH, Germany) and stored at $4^{\circ} \mathrm{C}$. To create a working stock, Pam3Cys was dissolved in sterile endotoxin-free physiological solution to give a final concentration of $1 \mathrm{mg} / \mathrm{mL}$ and aliquots were stored at $-20^{\circ} \mathrm{C}$. In use aliquots were stored at $4^{\circ} \mathrm{C}$ for a maximum of 3 weeks.

\section{Polyinosinic:Polycytidylic Acid (Poly I:C)}

Low molecular weight Poly I:C, a synthetic analog of dsRNA (148), was purchased as lyophilised powder from InvivoGen (SAN Diego, CA, USA) and dissolved in sterile endotoxin-free physiological solution as provided by the supplier at a stock concentration of $20 \mathrm{mg} / \mathrm{mL}$. Aliquots were stored at $-20^{\circ} \mathrm{C}$ and in use aliquots were stored at $4{ }^{\circ} \mathrm{C}$ for a maximum of 3 weeks.

\subsubsection{Antibodies and Fluorophores}

Antibodies used to minimise non-specific antibody binding

\begin{tabular}{|c|l|l|l|}
\hline Antigen & Clone & \multicolumn{1}{c|}{ Fluorophore } & \multicolumn{1}{c|}{ Source } \\
\hline Anti-Fc $\gamma$ RII/III & $2.4 \mathrm{G} 2$ & None & Purified in-house \\
\hline
\end{tabular}

Antibodies used for surface marker staining of DCs

\begin{tabular}{|l|l|l|l|}
\hline \multicolumn{1}{|c|}{ Antigen } & \multicolumn{1}{|c|}{ Clone } & \multicolumn{1}{c|}{ Fluorophore } & \multicolumn{1}{c|}{ Source } \\
\hline B220 & RA3-6B2 & $\begin{array}{l}\text { Pacific Blue, } \\
\text { PerCP }\end{array}$ & BD Pharmingen, CA, USA \\
\hline B220 & RA3-6B2 & Biotin, FITC & Purified in-house \\
\hline CD11b & M1/70 & Biotin & eBioscience, San Diego, CA, USA \\
\hline CD11b & M1/70 & PerCP-Cy5.5 & BD Pharmingen, CA, USA \\
\hline CD11c & N418 & $\begin{array}{l}\text { Alexa Fluor 647, } \\
\text { FITC }\end{array}$ & Purified in-house \\
\hline CD11c & HL3 & PE-Cy7 & BD Pharmingen, CA, USA \\
\hline
\end{tabular}




\begin{tabular}{|l|l|l|l|}
\hline CD24 & M1/69 & FITC & Biolegend, San Diego, CA, USA \\
\hline CD24 & M1/69 & PE & eBioscience, San Diego, CA, USA \\
\hline CD40 & $3 / 23$ & PE & BD Pharmingen, CA, USA \\
\hline CD80 & 16-10A1 & Biotin, PE & BD Pharmingen, CA, USA \\
\hline CD86 & GL1 & FITC & Purified in-house \\
\hline CD86 & GL1 & V450 & BD Pharmingen, CA, USA \\
\hline $\begin{array}{l}\text { MHC class II (I- } \\
\text { A/I-E) }\end{array}$ & 3JP & $\begin{array}{l}\text { Alexa Fluor 647, } \\
\text { Biotin }\end{array}$ & Purified in-house \\
\hline
\end{tabular}

Antibodies used for pDC depletion by magnetic cell separation (MACS)

\begin{tabular}{|l|c|l|l|}
\hline \multicolumn{1}{|c|}{ Antigen } & Clone & \multicolumn{1}{c|}{ Fluorophore } & \multicolumn{1}{c|}{ Source } \\
\hline B220 & RA3-6B2 & Biotin & Purified in-house \\
\hline
\end{tabular}

Antibodies used for pDC depletion using BioMag $\mathbb{B}$ beads

\begin{tabular}{|c|c|l|l|}
\hline Antigen & Clone & \multicolumn{1}{|c|}{ Fluorophore } & Source \\
\hline B220 & RA3-6B2 & PerCP & BD Pharmingen, CA, USA \\
\hline
\end{tabular}

Antibodies used for cDC flow cytometry sorting

\begin{tabular}{|l|l|l|l|}
\hline \multicolumn{1}{|c|}{ Antigen } & \multicolumn{1}{c|}{ Clone } & \multicolumn{1}{c|}{ Fluorophore } & \multicolumn{1}{c|}{ Source } \\
\hline B220 & RA3-6B2 & FITC & Purified in-house \\
\hline CD11c & N418 & Alexa Fluor 647 & Purified in-house \\
\hline
\end{tabular}

Antibodies used for intracellular staining

\begin{tabular}{|l|l|l|l|}
\hline \multicolumn{1}{|c|}{ Antigen } & \multicolumn{1}{c|}{ Clone } & \multicolumn{1}{c|}{ Fluorophore } & \multicolumn{1}{c|}{ Source } \\
\hline IL-12p40/70 & C15.6 & PE & BD Pharmingen, CA, USA \\
\hline $\begin{array}{l}\text { Rat IgG1 } \kappa \text { iso- } \\
\text { type control }\end{array}$ & R3-34 & PE & BD Pharmingen, CA, USA \\
\hline TNF- $\alpha$ & $\begin{array}{l}\text { MP6- } \\
\text { XT22 }\end{array}$ & FITC & eBioscience, San Diego, CA, USA \\
\hline $\begin{array}{l}\text { Rat IgG1 isotype } \\
\text { control }\end{array}$ & EBRG1 & FITC & eBioscience, San Diego, CA, USA \\
\hline
\end{tabular}


Antibodies used for immunofluorescence staining

\begin{tabular}{|l|l|l|l|}
\hline \multicolumn{1}{|c|}{ Antigen } & \multicolumn{1}{c|}{ Clone } & \multicolumn{1}{c|}{ Fluorophore } & \multicolumn{1}{c|}{ Source } \\
\hline B220 & $6 \mathrm{~B} 2$ & Biotin & Purified in-house \\
\hline CD11c & N418 & Alexa Fluor 647 & Purified in-house \\
\hline
\end{tabular}

The primary biotinylated antibody was further stained with a secondary antibody, Streptavidin Alexa Fluor 555.

\section{Streptavidin (SA)- Fluorophore Conjugates}

SA-APC, SA-FITC, SA-PE and SA-PerCP were purchased from BD Pharmingen, CA, USA and stored at $4{ }^{\circ} \mathrm{C}$. SA-Alexa Fluor 555 was purchased from Invitrogen (Auckland, $\mathrm{NZ}$ ) and stored in aliquots at $-20^{\circ} \mathrm{C}$.

\section{Cell viability dyes}

4,6-Diamindino-2-Phenylindole Dihydrochloride (DAPI) was purchased as lyophilised powder from Invitrogen (Auckland, NZ) and dissolved in $\mathrm{dH}_{2} \mathrm{O}$ to a concentration of $5 \mathrm{mg} / \mathrm{mL}$. This solution was then further diluted to a stock concentration of $200 \mu \mathrm{g} / \mathrm{mL}$ in FACS buffer and stored in aliquots at $4^{\circ} \mathrm{C}$. When used, DAPI was diluted in FACS buffer at 1:1000 dilution and $150 \mu \mathrm{L}$ were added to cells.

LIVE/DEAD® Fixable Blue Dead Cell Stain Kit, was purchased from Invitrogen (Auckland, NZ) and stored at $-20^{\circ} \mathrm{C}$. The lyophilised dye powder was dissolved in $50 \mu \mathrm{L}$ DMSO per vial and stored at $4^{\circ} \mathrm{C}$. When used, the viability dye was diluted in FACS buffer at 1:500 dilution and 50 $\mu \mathrm{L}$ were added to cells.

Propidium Iodide (PI) was purchased from BD Pharmingen (San Diego, CA, USA) in liquid form and stored at $4^{\circ} \mathrm{C}$. 


\subsubsection{Proteins and peptides}

Ovalbumin protein from chicken egg white (OVA) was purchased from Sigma-Aldrich NZ Ltd, Auckland, NZ. The OVA peptides SIINFEKL $\left(\mathrm{OVA}_{257-264}\right)$ and ISQAVHAAHAEINEAGR $\left(\mathrm{OVA}_{323-339)}\right)$ were purchased from Mimotopes Pty Ltd (Clayton, VA, Australia). Hereafter, the term ISQ will be used in referral to the ISQAVHAAHAEINEAGR peptides.

\subsubsection{Assay buffer compositions}

FACS buffer

Foetal bovine serum (FBS)

Sodium azide

EDTA

\section{Final concentration}

$$
\begin{aligned}
& 0.2 \% \\
& 0.01 \% \\
& 10 \mathrm{mM}
\end{aligned}
$$

All reagents were added to $1 \mathrm{~L}$ D-PBS (made in-house) and stored at $4{ }^{\circ} \mathrm{C}$.

\section{Wuerzburger buffer}

Foetal bovine serum (FBS)

DNAse I

EDTA

\section{Final concentration}

$0.1 \%$

$10 \mu \mathrm{g} / \mathrm{ml}$

$5 \mathrm{mM}$

All reagents were added to $500 \mathrm{~mL}$ PBS and stored at $4{ }^{\circ} \mathrm{C}$.

\section{Bioplex assay buffer}

Bovine serum albumin (BSA)

Tween ${ }^{\circledR} 20$

Sodium azide

EDTA

\section{Final concentration}

$$
\begin{aligned}
& 0.1 \% \\
& 0.05 \% \\
& 0.005 \% \\
& 2.5 \mathrm{mM}
\end{aligned}
$$

All reagents were added to $500 \mathrm{~mL}$ PBS and stored at $4{ }^{\circ} \mathrm{C}$. 


\subsubsection{Kits}

Biotin/Avidin blocking kit

Bioplex bead array kit (IL-12p70, IL-10, IL-6, TNF- $\alpha$,) Invitrogen, NZ

Diff-Quik kit

BD Cytofix/Cytoperm ${ }^{\mathrm{TM}}$ Plus (with GolgiStop) kit
Invitrogen, NZ

Dade Behring,

Newark, USA

BD Bioscience,

\subsection{Tumour cell lines}

The B16.OVA melanoma tumour cell line was generated by Dr Edith Lord and Dr John G, Frelinger, University of Rochester, Rochester, NY and kindly provided by Dr Roslyn Kemp and Dr Dick Dutton, Trudeau Institute, NY, USA.

\subsection{Mice}

\subsubsection{Maintenance and ethical approvals}

All mice were bred and maintained in the Biomedical Research Unit of the Malaghan Institute of Medical Research. All experimental procedures were approved by the Victoria University Animal Ethics Committee and carried out in accordance with institutional guidelines, under the license code 2009R8M (Use of mouse tissue for multiple research projects within the Malaghan Institute) or 2010R1M (Tumour immunity and intratumoral environment). All mice used for experiments were male or female, aged between 6-11 weeks and sex matched within each experiment. 


\subsubsection{Mouse strains}

C57BL/6 (C57) breeding pairs were originally obtained from the Jackson Laboratories (Bar Harbour, ME, USA) and bred at the Malaghan Institute of Medical Research, Wellington, NZ, by brother X sister mating.

OTI and OTII mice $(228,229)$ expressing transgenic T cell receptor (TCR) specific for ovalbumin (OVA) 257-264 presented on $\mathrm{H}_{2}-\mathrm{K}^{\mathrm{b}}$ or (OVA)323-339 presented on $\mathrm{I}^{-} \mathrm{A}^{\mathrm{b}}$ respectively were obtained from Dr Sarah Hook, School of Pharmacy, Dunedin, NZ, with the permission of Prof Frank Carbone, Melbourne University, Australia.

OTI x B6.SJL-Ptprca and OT-II x B6.SJL-Ptprca congenic mice were bred in-house, by crossing OTI mice (CD45.2+) or OTII (CD45.1+) with B6 congenic mice for one generation.

TLR4-/- mice were kindly provided by Dr Shizuo Akira, Hyogo College of Medicine, Japan (230).

\subsection{Methods}

\subsubsection{General cell culture}

All cells were cultured in cIMDM at $37^{\circ} \mathrm{C}$ in $5 \% \mathrm{CO}_{2}$ and $95 \%$ humidity.

Tumour cell lines were cultured in cIMDM supplemented with $0.5 \mathrm{mg} / \mathrm{mL}$ G418 (GIBCO, Auckland, NZ) in $200 \mathrm{~mL}$ culture flasks. To harvest the cells, $3 \mathrm{~mL}$ of Trypsin/EDTA was added and incubated for 1 minute to detach the cells from the flask. Following incubation, an equal volume of cIMDM was added to stop proteolysis and cells were washed three times in IMDM before resuspending at $1 \times 10^{6}$ cells $/ \mathrm{mL}$ and injecting into mice. 


\subsubsection{Dendritic cell culturing and manipulation}

\subsubsection{Extraction of bone marrow cells}

C57BL/6 mice were sacrificed by gas asphyxiation followed by cervical dislocation. Hind legs were detached from the hip and muscle and connective tissue were removed from the femur and tibia and collected in IMDM. End of the bones were snipped away and BM cells were flushed into a $50 \mathrm{~mL}$ falcon tube using a 25 -gauge needle and a $10 \mathrm{~mL}$ syringe filled with IMDM. Cell clumps were disrupted by vigorous pipetting and the cell suspension was strained through a $70 \mu \mathrm{m}$ cell strainer. Live cells were identified using Trypan blue dye (GIBCO, Invitrogen, Auckland, NZ) exclusion and counted using a haemocytometer. Cells were then resuspended at the appropriate concentration.

\subsubsection{Generating Flt3L-DCs}

BM cells were obtained as described in 2.4.2.1. Following cell counting, cells were pelleted by centrifugation at $300 \mathrm{x} \mathrm{g}$ for 10 minutes and resuspended at $1 \times 10^{6}$ cells $/ \mathrm{mL}$ in cIMDM supplemented with Flt3L at the optimised concentration $(2-4 \%)$. $5 \mathrm{~mL}$ were pipetted into each well of a 6-well plate and incubated for 9 days. Cells were supplemented with fresh nutrients on days 3, 6 and 9 by replacing $2 \mathrm{~mL}$ of medium from each well with cIMDM containing 0.05-0.10\% Flt3L (231).

\subsubsection{Generating GMCSF/IL-4 DCs}

BM cells were obtained as described in 2.4.2.1. Following cell counting, cells were pelleted by centrifugation at $300 \mathrm{x}$ g for 10 minutes and resuspended at $2 \times 10^{6}$ cells per 5 $\mathrm{mL}$ in cIMDM containing 10ng/ml GM-CSF and 20ng/ml IL-4 (232). 5mL were pipetted into each well of a 6-well plate and incubated for 7 days. On days 3 and 5, approximately $2 \mathrm{~mL}$ from each well was removed and fresh cIMDM supplemented with 10ng/ml GMCSF and 20ng/ml IL-4 was added to replenish nutrients. 


\subsubsection{Inducing DC activation}

\section{Flt3L-DC activation}

Flt3L-DCs were stimulated with various TLR agonists on day 9 for 24 hours or on day 10 if the period of stimulation was less. The final concentrations of the TLR agonists used were: $\mathrm{CpG}(2 \mu \mathrm{g} / \mathrm{mL})$, Poly I:C $(50 \mu \mathrm{g} / \mathrm{mL})$ and Pam3Cys $(1 \mu \mathrm{g} / \mathrm{mL})$. The concentration of LPS used varied at either $100 \mathrm{ng} / \mathrm{mL}, 250 \mathrm{ng} / \mathrm{mL}$ or $500 \mathrm{ng} / \mathrm{mL}$ and are indicated within Chapter 2 and 3. During the time of stimulation, Flt3L-DCs were incubated at $37^{\circ} \mathrm{C}$. Following stimulation, non-adherent cells were harvested by gentle pipetting, centrifuged at $300 \mathrm{x}$ g for 10 minutes and resuspended at the appropriate concentration for subsequent use.

\section{$\underline{\text { GMCSF/IL-4 DC activation }}$}

GMCSF/IL-4 DCs were stimulated with LPS alone or in combination with Pam3Cys. Days in which DCs received the stimulus and periods of incubation varied. As described in Chapter 3, GMCSF/IL-4 DCs were stimulated for 6 hours on day 7 of cell culture with LPS at concentrations of $100 \mathrm{ng} / \mathrm{mL}, 250 \mathrm{ng} / \mathrm{mL}$ and $500 \mathrm{ng} / \mathrm{mL}$. Cells were then incubated at $37^{\circ} \mathrm{C}$ for 24 hours. In Chapter $4, \mathrm{GMCSF} / \mathrm{IL}-4 \mathrm{DCs}$ were stimulated on day 6 of cell culture with a combination of LPS $(100 \mathrm{ng} / \mathrm{mL})$ and Pam3Cys $(100 \mathrm{ng} / \mathrm{mL})$ and cells were then incubated at $37^{\circ} \mathrm{C}$ for 24 hours. Following stimulation non-adherent cells were harvested by gentle pipetting, centrifuged at $300 \mathrm{x} g$ for 10 minutes and resuspended at the appropriate concentration for subsequent use.

\subsubsection{Loading Flt3L-DCs with peptides for in vitro assays}

For in vitro assays, Flt3L-DCs were loaded with peptides following stimulation with TLR agonists as described in 2.4.2.4. DCs were harvested from culture and resuspended at $1 \mathrm{x}$ $10^{6}$ cells $/ \mathrm{mL}$. SIINFEKL or ISQ peptides were added into the DC suspensions at $0.3 \mathrm{nM}$ or $0.5 \mathrm{mM}$ respectively and incubated at $37{ }^{\circ} \mathrm{C}$ for 2 hours. After incubation, DCs were washed twice in cIMDM and resuspended at the appropriate concentration in CIMDM for further use. 
For in vivo experiments involving DC vaccination, the same procedure was performed as

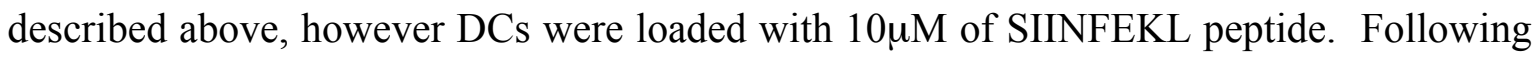
a 2 hour incubation, DCs were washed three times with IMDM and resuspended at $1 \times 10^{6}$ cells/mL in IMDM.

\subsubsection{Loading of Flt3L-DCs with OVA protein}

OVA protein loading occurred on day 8 of cell culture. OVA protein powder was weighed in a tissue culture dish and dissolved in PBS to make a stock concentration of $40 \mathrm{mg} / \mathrm{ml}$. The protein solution was then sterilized by filtering through a $32 \mathrm{~mm}$ syringe filter and added to the DC cultures at a final concentration of $0.5 \mathrm{mg} / \mathrm{mL}$. Cells were then left to incubate overnight (14-15 hours).

\subsubsection{Treatment of Flt3L and GMCSF/IL-4 DCs with 2-Deoxy-D- glucose}

Treatment with 2-DG occurred on day 10 for Flt3L and day 7 for GMCSF/IL-4 DC cultures. 2-DG was weighed out and resuspended to a working concentration of $25 \mathrm{mM}$ in cIMDM and set aside to dissolve at room temperature. The 2-DG solution was then sterilized by filtering through a $32 \mathrm{~mm}$ syringe filter and subsequently added to the DC cultures at final concentrations of $25 \mathrm{mM}, 12.5 \mathrm{mM}$ or $6.25 \mathrm{mM}$. DCs were incubated for 6 hours and then harvested, centrifuged at $300 \mathrm{x} g$ for 10 minutes and resuspended at the appropriate concentration for fluorescent antibody labelling (2.4.5.1).

\subsubsection{Cell purification/depletion and cell sorting}

\subsubsection{Preparation of lymph node and spleen suspensions}

Lymph nodes were punctured with a needle and then pressed through a $70 \mu \mathrm{m}$ cell strainer with a $1 \mathrm{ml}$ syringe plunger and flushed simultaneously with IMDM into 50mL falcon tubes. The cell suspension was then centrifuged at $300 \mathrm{x}$ g for 10 minutes, washed with Wuerzburger buffer and stored on ice until further use. 
Spleens were cut into small pieces using a pair of scissors. The spleens were disrupted by pressing with a $1 \mathrm{ml}$ syringe plunger through a $70 \mu \mathrm{m}$ cell strainer and flushed simultaneously with IMDM into 50mL falcon tubes. Splenocyte suspensions were treated with ACT buffer for $5 \mathrm{~min}$ at $37^{\circ} \mathrm{C}$ to lyse the red blood cells. Cells were then washed with Wuerzburger buffer and stored on ice until further use.

\subsubsection{Purification of $\mathrm{T}$ cells using magnetic cell separation (MACS)}

$\mathrm{CD}^{+} \mathrm{T}$ cells were enriched from the lymph nodes and spleens of naïve OT-II or OT-II B6.SJL-Ptprca mice, while $\mathrm{CD} 8^{+} \mathrm{T}$ cells were enriched from the lymph nodes and spleens of naïve OT-I or OT-I B6.SJL-Ptprca mice. Lymphocyte and splenocyte suspensions were prepared separately as described in 2.4.3.1. Both cell suspensions were pooled together and centrifuged at $300 \mathrm{x}$ g for 10 minutes and resuspended in Wuerzburger buffer. The cell mixture was filtered through a $70 \mu \mathrm{m}$ cells strainer, counted and resuspended at $1 \times 10^{7}$ cells per $90 \mu \mathrm{L}$ of Wuerzburger buffer. For $\mathrm{CD}^{+} \mathrm{T}$ cell enrichment, 10uL of anti-CD4 MACS microbeads were added per $1 \times 10^{7}$ cells. For $\mathrm{CD}^{+} \mathrm{T}$ cell enrichment, 10uL of antiCD8 MACS microbeads were added per $1 \times 10^{7}$ cells. The cell mixture was then incubated on ice for 15 minutes with regular mixing during the incubation. Following incubation, cells were washed by adding $1-2 \mathrm{~mL}$ of Wuerzburger buffer per $1 \times 10^{7}$ cells and then centrifuged at $300 \mathrm{x}$ g for 10 minutes to discard unbound microbeads. The cells were then resuspended to $100 \times 10^{6}$ cells $/ \mathrm{mL}$ in Wuerzburger buffer, passed through a $30 \mu \mathrm{m}$ MACS pre-separation filter and loaded on the AutoMACS machine for positive magnetic selection (Miltenyi Biotec GmbH, Germany). Lymphocytes were resuspended at $1 \times 10^{6}$ cells $/ \mathrm{mL}$ in cIMDM and left on ice until further use. Purification efficiency of $\mathrm{CD}^{+}$and $\mathrm{CD} 8^{+} \mathrm{T}$ cells was checked by flow fluorescent labelling of cells and analysed by flow cytometry (Appendix 1).

\subsubsection{Depletion of $\mathrm{B220}^{+} \mathrm{pDCs}$ using magnetic cell separation (MACS)}

Flt3L-DCs were harvested from culture on day 9 and centrifuged at $300 \mathrm{x} \mathrm{g}$ for 10 minutes. Cells were counted and resuspended at $1 \times 10^{7}$ cells $/ \mathrm{mL}$ in Wuerzburger buffer. AntiFc $\gamma$ RII/III antibody (2.4G2) was added to the cell suspension and incubated on ice for 10 
minutes to block Fc receptors. Biotinylated B220 antibody was added to the cell suspensions containing $2.4 \mathrm{G} 2$ and incubated for a further 15 minutes on ice. Following incubation, an equal volume of Wuerzburger buffer was added to wash the cells and remove excess antibody. The wash step was performed twice and cells were then resuspended at $1 \times 10^{7}$ cells per $90 \mu \mathrm{L}$ of Wuerzburger buffer. Streptavidin conjugated microbeads were added and cells were incubated on ice for 15 minutes. After incubation, cells were washed twice and resuspended at $1 \times 10^{8}$ cells $/ \mathrm{mL}$ in Wuerzburger buffer. The cells were then passed through a $30 \mu \mathrm{m}$ MACS pre-separation filter and loaded on the AutoMACS machine for negative magnetic selection (Miltenyi Biotec GmbH, Germany). Efficiency of pDC depletion was checked by flow fluorescent labelling of cells and analysed by flow cytometry (Appendix 2).

\subsubsection{Depletion of $\mathrm{B220}^{+}$pDCs using BioMag® beads}

Flt3L-DCs were harvested from culture on day 9 and centrifuged at $300 \mathrm{x}$ g for 10 minutes. Cells were counted and resuspended at $1 \times 10^{7}$ cells $/ \mathrm{mL}$ in Wuerzburger buffer. AntiFc $\gamma$ RII/III antibody (2.4G2) was added to the cell suspension and incubated on ice for 10 minutes to block Fc receptors. Antibody against B220 (Rat Anti-Mouse B220 PerCP) was added to the cell suspensions containing 2.4G2 and incubated for a further 15 minutes on ice. After incubation, cells were washed twice with Wuerzburger buffer to remove excess antibodies. Cells were re-counted and resuspended at $1 \times 10^{7}$ cells $/ \mathrm{mL}$ and BioMag® beads were subsequently added to the cell suspension at a ratio of 15 beads per cell. BioMag® beads work by specifically binding to cells labelled with rat mAb. The cells were then placed on the spinning wheel at $4^{\circ} \mathrm{C}$ and incubated for 30 minutes. Following incubation, cells were then washed twice and placed on the DynalMag-15 magnet (Invitrogen, USA) to trap the beads bound to the $\mathrm{B} 220^{+}$cells on the magnet. Cells of interest (cDCs) within the supernatant were collected.

\subsubsection{Fluorescence activated cell sorting (FACS) of Flt3L-DCs}

Flt3L-DCs were harvested from culture on day 9 and centrifuged at $300 \mathrm{xg}$ for 10 minutes. Cells were counted and resuspended at $5 \times 10^{6}$ cells $/ \mathrm{mL}$ in Wuerzburger buffer. Anti- 
Fc $\gamma$ RII/III antibody (2.4G2) was added to the cell suspension and incubated on ice for 10 minutes. Fluorescently conjugated antibody against the surface markers CD11c and B220 was added to the cell suspensions containing 2.4G2 and incubated for a further 15 minutes on ice. Cells were washed twice with Wuerzburger buffer and passed through a $30 \mu \mathrm{m}$ MACS pre-separation filter to remove cell clumps. Cells were then centrifuged at $300 \mathrm{xg}$ for 10 minutes, counted and resuspended at $6 \times 10^{6}$ cells $/ \mathrm{mL}$ in Wuerzburger buffer. Cells were sorted using a FACSVantage SE DiVa (Becton Dickinson, CA, USA). Unlabelled and single labelled samples for each fluorochrome were used to set the voltage and compensation parameters. Cells that stained positive for the markers CD11c and negative B220 were collected for further use.

\subsubsection{Assays of cell function}

\subsubsection{In vitro $\mathrm{T}$ cell proliferation assay}

In vitro $\mathrm{T}$ cell proliferation assays were performed for both $\mathrm{CD}^{+}$and $\mathrm{CD} 4^{+} \mathrm{T}$ cells. Flt3L-DCs were harvested from culture on day 9, depleted of $\mathrm{B} 220^{+} \mathrm{pDCs}$ and stimulated with TLR agonists as described in 2.4.3.3 and 2.4.2.4, respectively. DCs were then loaded with SIINFEKL or ISQ peptides as described in 2.4.2.5. During the 2 hour incubation with peptides, lymph nodes and spleens were harvested from OT-II and OT-I mice and made into cell suspensions as described in 2.4.3.1. The lymphocyte mixtures were then enriched for $\mathrm{CD}^{+}$and $\mathrm{CD}^{+} \mathrm{T}$ cells as described 2.4.3.2. Following peptide loading, DCs were resuspended at $0.1 \times 10^{6}$ cells $/ \mathrm{mL}$ in cIMDM and serially titrated onto 96-well culture plates. The initial starting concentration was $1 \times 10^{4}$ DCs per well and DCs were serially titrated at 2 fold dilutions. DCs loaded with OVA323-33 and SIINFEKL peptide received $\mathrm{CD}^{+}$and $\mathrm{CD}^{+} \mathrm{T}$ cells, respectively at $1 \times 10^{5}$ cells per well. Plates containing $\mathrm{CD} 8^{+} \mathrm{T}$ cells were then incubated for 48 hours at $37^{\circ} \mathrm{C}$, but those containing $\mathrm{CD} 4^{+} \mathrm{T}$ cells were incubated for $48-72$ hours at $37^{\circ} \mathrm{C}$. After the incubation, $1 \mu \mathrm{Ci}\left[{ }^{3} \mathrm{H}\right]$-thymidine was added into the wells and cells were incubated for another 18 hours. Following the incubation with thymidine, the cells were harvested using an automated cell harvester (Tomtec, CT, USA) onto Wallac Filters (Turku, Finland). Filters were dried and sealed in sample bags (Wallac) with $5 \mathrm{~mL}$ BetaScint scintillation fluid (Wallac). Thymidine incorporation was 
measured using Wallac 1450 MicrobetaPlus Liquid Scintillation Counter (PerkinElmer Life Sciences and Analytical Sciences, formerly Wallac Oy) and acquired using Wallac 1450 MicroBeta Windows Workstation ver. 2.70.004. Thymidine incorporation was used as a measure of proliferation.

\subsubsection{In vitro cross-presentation assay}

Flt3L-DCs were generated from TLR4-/- BM cells as described in 2.4.2.2 and loaded with OVA protein as described in 2.4.2.6. Following incubation with OVA protein, Flt3L-DCs were depleted of $\mathrm{B} 220^{+} \mathrm{pDCs}$ and stimulated with TLR agonists as described in 2.4.3.3 and 2.4.2.4, respectively. Cells were counted and resuspended at $0.1 \times 10^{6}$ cells $/ \mathrm{mL}$ and titrated onto 96-well plates. The initial starting concentration was $1 \times 10^{4} \mathrm{DCs}$ per well and DCs were serially titrated at 2 fold dilutions. $\mathrm{CD}^{+} \mathrm{T}$ cells that had been prepared earlier $(2.4 .3 .1,2.4 .3 .2)$ were added at $1 \times 10^{5}$ cells per well. Cells were then incubated for 2 days at $37{ }^{\circ} \mathrm{C}$. After the incubation, $1 \mu \mathrm{Ci}\left[{ }^{3} \mathrm{H}\right]$-thymidine was added into the wells and cells were incubated for another 18 hours. Cell harvesting and analysis was performed as described in 2.4.4.1.

\subsubsection{Detection of cytokine production from DC supernatant}

Flt3L-DCs were stimulated with TLR agonists as described in 2.4.2.4. Cells were then harvested following stimulation and centrifuged at $300 \mathrm{x}$ g for 10 minutes. Supernatants were then transferred into new $2 \mathrm{~mL}$ cryotubes and stored at $-20^{\circ} \mathrm{C}$ until further analysis. Levels of IL-12p70, TNF- $\alpha$ and IL-6 were determined using a multiplex cytokine detection kit (Invitrogen, NZ) according to the manufacturer's instructions. Briefly, a mixture of capture beads specific for each cytokine was prepared at $0.833 \mu \mathrm{L}$ of each bead per allocated well in bioplex assay buffer at a volume of $22.5 \mu \mathrm{L}$ per allocated well. $25 \mu \mathrm{L}$ of the bead mixture was then added to each well and the plate was washed twice with bioplex assay buffer by adding $200 \mu \mathrm{L}$ per well and removing by vacuum manifold (BioRad, USA). Supernantant samples were diluted at 1:2 in bioplex assay buffer and $50 \mu \mathrm{L}$ was added to the wells. The plate was then incubated with agitation for 2 hours at room temperature and then washed twice as described above. Biotinylated detection antibodies 
were made up at a 1:30 dilution and $25 \mu \mathrm{L}$ was added to the plate followed by 1 hour incubation with agitation at room temperature. The plate was then washed twice and incubated with $100 \mu \mathrm{L}$ of SA-PE for 30 minutes at room temperature. The plate was washed three times as described above and samples were then resuspended in $200 \mu \mathrm{L}$ of bioplex assay buffer and analysed using the Bio-Plex ${ }^{\mathrm{TM}}$ system. Cytokine concentrations were determined against commercial standards (Invitrogen, NZ) and calculated using the provided software (Bio-Plex manager software, Bio-Rad, USA).

\subsubsection{Fluorescent labelling of cells and flow cytometry}

\subsubsection{Detection of surface marker expression}

Single cell suspensions were counted, washed once in FACS buffer and resuspended at 1$2 \times 10^{6}$ cells $/ \mathrm{mL}$. Cells were then transferred onto 96-well plates and centrifuged at $320 \mathrm{xg}$ for 2 minutes to pellet the cells. The supernatant was discarded by tipping the plate up side down and flicking once and the pellets were resuspended by gentle vortexing. The cells were then incubated with anti-FcyRII/III (2.4G2) antibodies for 10 minutes on ice to block Fc receptors. After the 10 minute incubation, fluorochrome-conjugated antibodies against cell surface markers were added to the 2.4G2 containing cell suspension at the appropriate dilutions and the cells were incubated for a further 10 minutes on ice. Cells were then washed twice by adding $200 \mu \mathrm{L}$ of FACS buffer into each well, and centrifuged at $320 \mathrm{x} \mathrm{g}$ for 2 minutes. The supernatant was then discarded as described above. If a primary biotinylated antibody was used, the appropriate streptavidin-conjugated fluorochrome wasadded and incubated for 10 minutes on ice. Cells were then washed twice in FACS buffer as described above and resuspended in $200 \mu \mathrm{L}$ of FACS buffer for flow cytometry analysis. Where addition of a viability dye were appropriate, cells were resuspended in 150uL of FACS buffer and 150 $\mu \mathrm{L}$ diluted DAPI (2.1.5) was added 5-10 minutes before analysis on the flow cytometer. Alternatively, cells were resuspended in $200 \mathrm{uL}$ of FACS buffer and $1 \mu \mathrm{L}$ of a $75 \mu \mathrm{g} / \mathrm{mL}$ stock of PI was added 5-10 minutes before analysis. 


\subsubsection{Detection of intracellular cytokines}

Flt3L-DCs were stimulated with TLR agonists as described in 2.4.2.4. For the final 4 hours of incubation, GolgiStop ${ }^{\mathrm{TM}}$ alone or in combination with Brefeldin A was added to the cells in culture to inhibit cytokine secretion. The combination of GolgiStop ${ }^{\mathrm{TM}}$ and Brefeldin A was used for detection of TNF- $\alpha$. Following incubation, cells were then harvested and stained for live cells using Live/Dead fixable dye as described in 2.1.5. Cells were then washed twice in FACS buffer and labelled with antibodies against surface makers as described in 2.4.5.1. Following surface labelling, cells were washed twice in FACS buffer and then incubated with $200 \mu 1$ of BD Cytofix/Cytoperm solution for 20 min at $4{ }^{\circ} \mathrm{C}$. The cells were then washed twice in $1 \mathrm{x}$ BD Perm/Wash buffer by adding $200 \mu \mathrm{L}$ per well and centrifuging at $300 \mathrm{x}$ g for 10 minutes. The cells were then incubated with antibodies against cytokines, or the respective isotype control antibodies for $30 \mathrm{~min}$ on ice. After the $30 \mathrm{~min}$ incubation, the cells were washed twice in BD Perm/Wash buffer. To minimize background staining, cells were allowed to sit in the Perm/Wash buffer for 10 min before centrifugation. After a total of three washes, the cells were resuspended in 200-300 $\mu$ L FACS buffer and stored at $4{ }^{\circ} \mathrm{C}$ wrapped in tin foil. Samples were collected and stained at different times, but were analysed together on the flow cytometer.

\subsubsection{Acquisition and analysis}

Antibody-labelled cells were analysed on a FACSort, FACScalibur, or LSRII SORP flow cytometer (Becton-Dickson, CA, USA). Following data acquisition on the flow cytometer, analysis was performed using the FlowJo software (Tree Star, San Carlos, CA, USA). Live cells or whole fixed cells were identified on the basis of Forward Scatter (FSC) and Side Scatter (SSC). In some experiments where a viability dye was applicable, live cells were identified by measure of PI or DAPI exclusion. Unstained samples and samples stained with single fluorophores were used to calibrate the acquisition voltages and set compensation for spectral overlap between fluorophores. For experiments analysing intracellular cytokines, the appropriate matched isotype control antibodies were used to control for background fluorescence. 


\subsubsection{Histology}

\subsubsection{Preparation of Flt3L and GMCSF/IL-4 DCs for morphological analysis and immuno-fluorescence staining}

Flt3L and GMCSF/IL-4 DCs were generated as described in 2.4.2.2 and 2.4.2.3 and harvested on day 9 and 7, respectively. DCs from both populations were centrifuged at $300 \mathrm{x}$ g for 10 minutes and resuspended at $1 \times 10^{5}$ cells $/ \mathrm{mL}$ in PBS containing $10 \%$ FBS. Cell samples in a volume of $200 \mathrm{uL}$ were centrifuged for 8 minutes at $600 \mathrm{rpm}$ onto glass slides (LabServ, Auckland, NZ) using a Shandon Cytospin 4 cytocentrifuge (Thermo Scientific, Cheshire, UK). Cells were then air dried for 1 hour.

\subsubsection{Morphological analysis of Flt3L and GMCSF/IL-4 DCs}

DCs were prepared as described in 2.4.6.1. Cells were then fixed for five seconds in DiffQuik Fixative $(1.8 \mathrm{mg} / \mathrm{mL}$ Triarylmethane dye methyl alcohol), stained for ten seconds in Diff-Quik Solution I (1g/L Xanthine dye) and then stained for seven seconds in Diff-Quik Solution II (0.625g/L Azure A, 0.625g/L Methylene blue). Cells were air dried for 1 hour and subsequently photographed by light microscopy using an Olympus BX51 microscope (Olympus, Auckland, NZ).

\subsubsection{Immunofluorescence staining of Flt3L-DCs}

DCs were prepared as described in 2.4.6.1. Cells were then fixed with a fixative solution containing 50\% methanol and 50\% acetone. After 10 minutes, cells were washed by rinsing the slide with PBS and non-specific binding sites were blocked by incubation with PBS supplemented with $10 \%$ FBS for 30 minutes at $37^{\circ} \mathrm{C}$. Cells were then washed as described above and internal cell biotin block was performed using a Biotin/Avidin blocking kit (Invitrogen, NZ) according to the manufacturer's protocol. Flourochromeconjugated CD11c Ab were made at the appropriate dilution in FACS buffer and 100uL was added over the cells and incubated in the dark for 1 hour at room temperature. Cells were then washed as described above and stained with biotinylated B220 antibody and incubated in the dark for 1 hour at room temperature. Following incubation, DCs were 
then washed again and stained with the secondary antibody Alexa Flour 555 and incubated in the dark for 30 minutes. Cells were washed with PBS and excess PBS was removed by blotting. Slides were further air dried in the dark for 30 minutes and a drop of ProLong Gold anti-fade with DAPI (Invitrogen) was applied onto the slide and mounted with a cover slip (Bio-lab Ltd, Auckland, NZ). Slides were examined using an Olympus BX51 (Olympus, Auckland, NZ) fluorescent microscope and images were visualised using a software as described in 2.4.6.4.

\subsubsection{Microscope image acquisition and analysis}

Microscope images of cells were acquired and optimised using AnalySIS Life Imaging Software (Olympus Soft Imaging Solutions GmbH, Münster, Germany). Photoshop software (Adobe Systems Inc, San Jose, CA, USA) was used on microscopic images for analysis.

\subsubsection{DC vaccination or tumour challenge}

\subsubsection{DC vaccination}

Flt3L-DCs and GMCSF/IL-4 DCs were generated as described in 2.4.2.2 and 2.4.2.3 respectively. On day 9 of cell culture, Flt3L-DCs were depleted of B220 ${ }^{+}$pDCs as described in 2.4.3.3. Following depletion, Flt3L-cDCs were placed back into culture and stimulated with TLR agonists as described in 2.4.2.4. GMCSF/IL-4 DCs were also stimulated with TLR agonists on day 6 as described in 2.4.2.4. Both DC populations were then loaded with SIINFEKL peptide as described in 2.4.2.5 and resuspended in IMDM at $1 \times 10^{6}$ cells $/ \mathrm{mL}$. DCs were injected s.c. into the right flank of C57BL/6 mice at varying DC numbers, as indicated in Chapter 4.

\subsubsection{Challenge with B16.OVA melanoma cells}

Mice were injected s.c. into the left flank with $1 \times 10^{5}$ B16.OVA melanoma cells. Tumours in untreated control mice were palpable from 8-10 days following tumour challenge and 
tumour growth was then checked every 2 days. Bisecting diameters were measured using Mitutoyo calipers. To account for the thickness of the skin, $1 \mathrm{~mm}$ was subtracted from each diameter measurement. Mice were euthanized when tumour size reached 150$200 \mathrm{~mm}^{2}$.

\subsection{Statistical analysis}

Non-parametric tests were chosen when analysing biological data, which does not assume that the data are sampled from a Gaussian distribution. Tests for normal distribution were not applicable with experiments presented in this thesis due to a small sample size $(\leq 3)$. To test for statistical significance using a non-parametric test, the Kruskal-Wallis with Dunn's multiple comparison tests were used in experiments for which there were two or more groups. The $\mathrm{P}$ values tend to be higher within non-parametric tests, thus statistical significance was not detected throughout many of the experiments presented within this thesis. All statistical analysis was carried out with the software GraphPad Prism (GraphPad Software Inc. San Diego, CA, USA). 


\section{Chapter 3.}

\section{Characterisation of Flt3L-DCs and evaluation of their response to stimulation with TLR agonists}




\subsection{Introduction}

As described in Chapter 1, the culture of precursor cells in Flt3L generates DCs that reproduce the heterogeneity observed in lymphoid organs, and differ substantially in phenotype and morphology from DCs generated with GMCSF/IL-4 (214, 231). Differences among the DCs that are generated using these culture methods are an important factor to consider, especially when implementing DCs as an immunotherapeutic tool. Thus, to assess the potential applicability of Flt3L-DCs for immunotherapy against cancer, we established a culture method within our laboratory that allows for the in vitro generation of Flt3L-DCs from murine BM precursors. This culture system is modelled from studies reported by other authors on Flt3L-DCs $(214,225,231)$, but will allow for the validation of results from those studies and, in addition, will be further extended within our system. Areas in which previous work will be further extended are discussed as follows.

\subsubsection{DC activation with TLR agonist combinations}

Most studies so far have analysed the activation of Flt3L-DCs that is induced by stimulation with single TLR agonists. Results have shown that upon TLR stimulation, Flt3L-DCs are capable of up-regulating co-stimulatory molecules and secretion of proinflammatory cytokines $(214,233,234)$. However, there is currently no published data exploring the effects of combined TLR ligation to enhance activation of Flt3L-DCs. Activation of GM-CSF DCs by selected TLR agonist combinations has been shown to induce pro-inflammatory cytokine secretion and expression of co-stimulatory molecules superior to DCs activated by one TLR agonist alone $(235,236)$. Napolitani et al first demonstrated that combined TLR stimulation with a TLR3/4 and TLR7/9 agonist augmented IL-12 production by GM-CSF/IL-4 DCs. The enhanced activation that was observed from combining the TLR agonists, was described as syngergistic activation (235). Soon after, Wager et al showed that synergistic activation of GM-CSF DCs was due to the combined triggering of differential signalling pathways within the cell, specifically the activation of MyD88 and TRIF adapter molecules (236). On engagement with their ligands, TLRs recruit specific adapter molecules that propagate downstream signalling (237). The pathway that uses the adapter molecule MyD88 leads to early activation of the 
transcription factor NF-אB and the production of inflammatory cytokines (238). In contrast, the pathway that utilises the adapter molecule TRIF leads to activation of the transcription factor IRF3 and late activation of NF- $\mathrm{\kappa B}$, thus leading to the induction of both type 1 interferons and inflammatory cytokines. With the exception of TLR3, which signals exclusively through TRIF, and TLR4, which can signal through TRIF and MyD88, all other TLRs that have been characterised to date signal through the MyD88 pathway (237, 239). Therefore, stimulation of Flt3L-DCs by the combined triggering of MyD88dependent and independent (TRIF) signalling pathways could potentially result in improved DC activation. Ultimately, this may form the basis for improved methods for Flt3L-DC activation. Figure 3.1 depicts a simplified overview of the TLRs and the respective agonists that were used within this study to stimulate Flt3L-DCs.

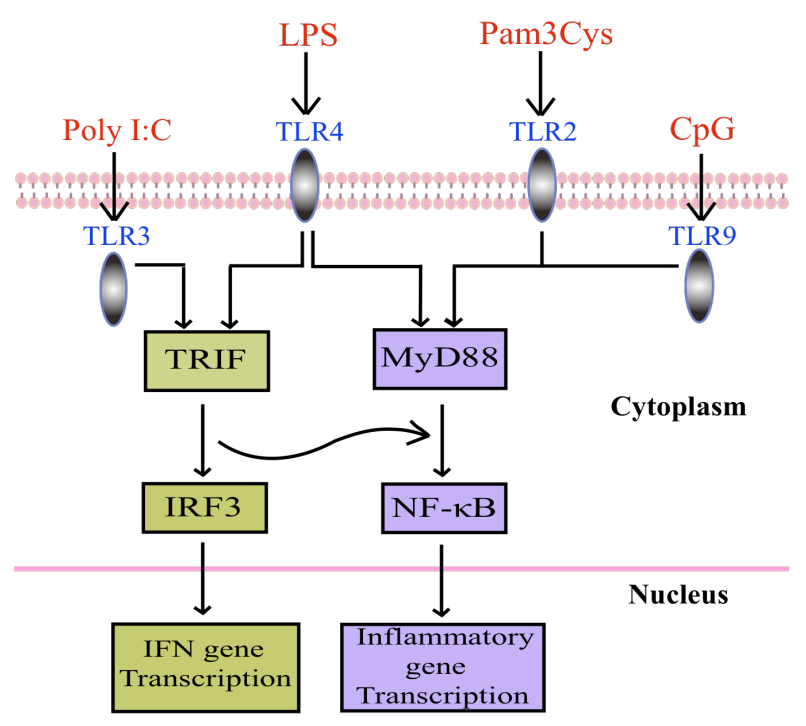

Figure 3.1: Overview of the TLR agonists used within this study and the corresponding signalling pathways. The TLR agonists (red) and corresponding TLRs (blue) that were used within this study are shown. The curved arrow represents putative connection between MyD88independent (green) and MyD88-dependent (purple) pathways used by TLR3. Adapted from Bagchi et al (240) 


\subsubsection{Metabolic requirements for cellular activation}

Immune cells are in constant need of energy in order to carry out housekeeping or immune specific functions (241). To fuel these functions, nutrients such as glucose, amino acids and fatty acids are taken up and degraded into intermediates, thereby providing the cell with a supply of metabolic substrates that can be used to generate energy in the form of ATP (242).

In $\mathrm{T}$ cells, earlier studies have brought insight into the regulation of energy metabolism between the resting and activated state. In the resting state, $\mathrm{T}$ cells have fewer bioenergetic demands relative to the activated state and devote a majority of energy metabolism to maintain housekeeping functions (241-243). The intracellular pool of ATP that is generated in resting $\mathrm{T}$ cells is derived from metabolic processes involving glycolysis and oxidative phosphorylation. During glycolysis, glucose is taken up and subsequently degraded to form pyruvate, which is further used as a substrate for energy metabolism via oxidative phosphorylation (242) (Figure 3.2A). Furthermore, other energy sources, such as fatty acids and amino acids can be taken up and degraded within the mitochondria, also generating intermediates maintaining ATP production (242). However, the transition from a resting to an activated state is an energetically demanding process. Therefore, upon activation, cellular metabolism is reprogrammed towards aerobic glycolysis in order to support cellular proliferation $(243,244)$. The advantage of this metabolic switch does not seem apparent as glycolysis yields less energy compared to oxidative phosphorylation. However, ATP is produced substantially faster, allowing T cells to meet the bioenergetic demands of activation $(242,243)$. As a result of this switch, continued ATP production becomes progressively more dependent on the degradation of glucose in the cytosol by glycolysis. Ultimately, aerobic glycolysis drives the formation of pyruvate into lactate in order to regenerate the cells pool of $\mathrm{NAD}^{+}$, that is needed as an electron acceptor to maintain cytosolic glucose metabolism (Figure 3.2B). 
A)

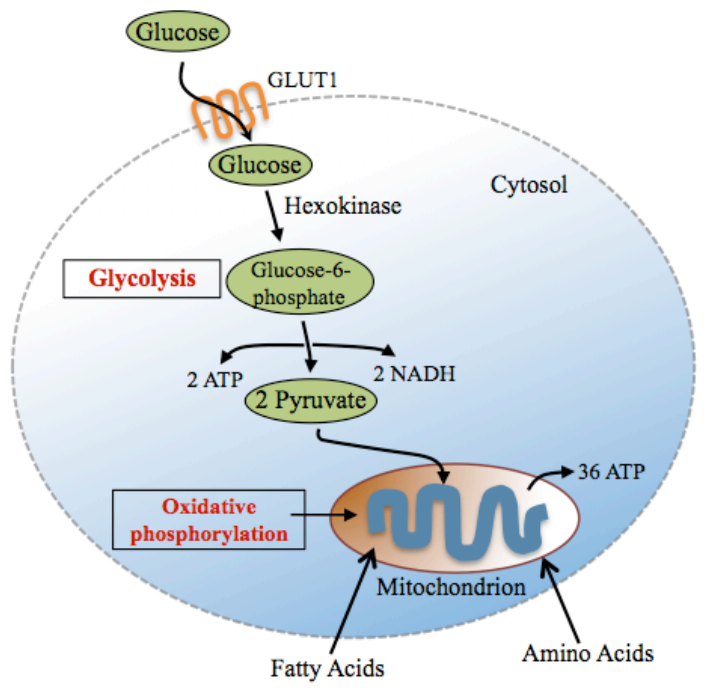

B)

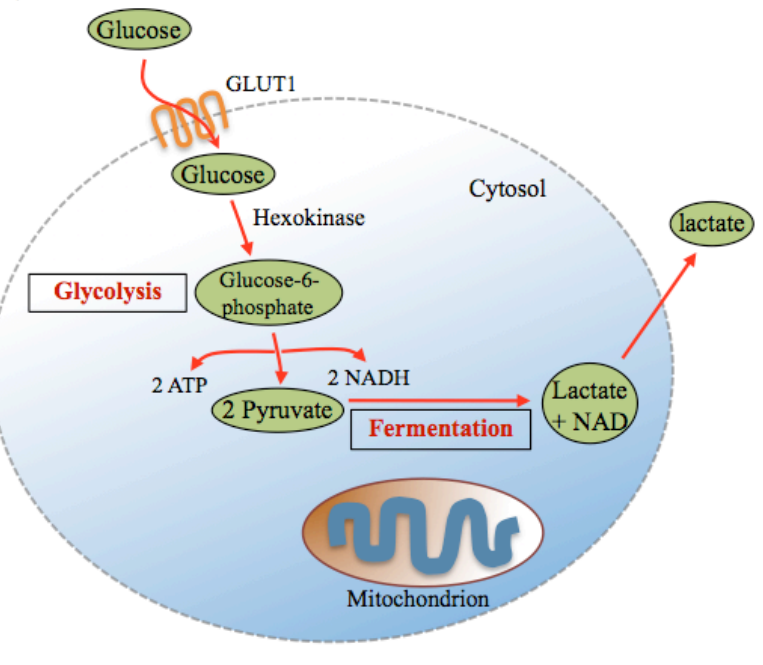

Figure 3.2: Resting and activated $T$ cells display distinct metabolic signatures. A)

Resting $\mathrm{T}$ cells derive the majority of their ATP through glycolysis and mitochondrial oxidative phosphorylation. Glucose is taken up by the glucose transporter 1 (GLUT1), where it is converted first to glucose-6-phosphate (G-6-P) by the enzyme hexokinase. G-6-P is further processed within the glycolytic pathway (additional enzymatic reactions not shown), generating two molecules each of pyruvate, NADH and ATP, per molecule of glucose. Pyruvate is further utilised in oxidative phosphorylation. Nutrients such as fatty acids and amino acids can also be taken up and degraded within the mitochondria, thus generating further intermediates for ATP production. Oxidative phosphorylation generates 36 ATP in total. B) Upon activation, T cells switch from oxidative phosphorylation to aerobic glycolysis. ATP is generated much faster, thus sustaining the bioenergetic demands of activation (red arrows). In aerobic glycolysis, pyruvate is reduced to lactate by fermentation, a reaction in which permits the regeneration of $\mathrm{NAD}^{+}$needed to maintain glycolysis. Adapted from Fox et al (242).

There is now evidence suggesting that DCs undertake the same metabolic conversion after exposure to TLR agonists. To determine whether TLR activation has effects on glycolytic metabolism, Krawczyk et al performed metabolic assays on in vitro derived GM-CSF DCs that were cultured in media supplemented with glucose (245). It was observed that TLR stimulated DCs had decreased mitochondrial-dependent oxygen consumption, despite the availability of oxygen and the rate of mitochondrial $\beta$-oxidation of fatty acids was also suppressed. TLR stimulated GM-CSF DCs had an increase in glycolytic rate compared to resting controls and inhibition of glycolytic metabolism resulted in impaired ability for the TLR treated DCs to up-regulate activation markers (245). Additionally, DCs subjected to glucose limitation for 24 hours, during the time of stimulation were unable to survive and 
underwent apoptosis. Unlike that of T cells, an increase in glycolytic metabolism due to TLR signalling does not promote DC proliferation. This implies that the increase in glycolysis triggered by TLRs, serves to deliver essential nutrients required for DC survival (245).

Collectively, these findings by Krawczyk et al suggested that the initiation of glycolytic metabolism at the time of activation is important for full DC maturation and subsequent survival. Thus, stimulated DCs, particularly GM-CSF DCs, must redirect their metabolic pathway from mitochondrial metabolism towards aerobic glycolysis. The type of metabolism that is utilised by Flt3L-DCs during activation has yet to be established. 


\subsection{Aims}

The aims of this chapter were to set up a culture system for Flt3L-DCs and to characterise the DCs that are generated. Characterisation was further extended by the analysis of TLR induced activation and of glycolytic metabolism.

Specifically, the aims were

1. To assess Flt3L-DC cell morphology and phenotype.

2. To determine the effect of stimulation with single and combined TLR agonists on the maturation of Flt3L-DCs.

3. To evaluate whether glycolytic metabolism is required for TLR mediated activation of Flt3L-DCs.

\subsection{Results}

\subsubsection{Morphological characteristics of Flt3L-DCs}

To generate Flt3L-DCs within our system, we established a culture method as described in Chapter 2. Briefly, BM cells were extracted from C57BL/6 mice and cultured with media containing the cytokine Flt3L for 9 days. Nutrients were replenished every 3 days with fresh media containing Flt3L. On day 9 of cell culture, Flt3L-DCs were harvested or treated with stimuli for further analysis. The cytokine Flt3L was generated and used at optimal concentrations as described in Chapter 2 (2.1.3).

According to the literature $(4,7)$, a comparative analysis of GMCSF/IL-4 and Flt3L-DCs highlighted a difference not only in phenotype, but also in cellular morphology between these two populations. By light microscopy, Flt3L-DCs are reported to be smaller in size compared to GMCSF/IL-4 DCs due to a lower amount of cytoplasm $(214,231)$. Thus, Flt3L- DCs were assessed morphologically in order to determine if similar features were reproduced within our culture system. GMCSF/IL-4 DCs were also generated (Chapter 2, 2.4.2.1, 2.4.2.3) to allow for comparison with Flt3L-DCs. On the final day of cell culture, 
Flt3L and GMCSF/IL-4 DCs were harvested, centrifuged onto glass slides, fixed and stained with a Diff-Quik staining kit. The cells were then examined under the microscope. On separate occasions, Flt3L and GMCSF/IL-4 DCs were also photographed directly in culture plates. A difference in size between the two DC populations was difficult to determine by cytospins (Figure 3.3A, 3.3B). However, by direct photography of DCs in culture, a difference in size between the two DC types was observed (Figure 3.3C, 3.3D). This result indicated that Flt3L-DCs generated using the culture method were smaller in size as described within the literature $(6,7)$.
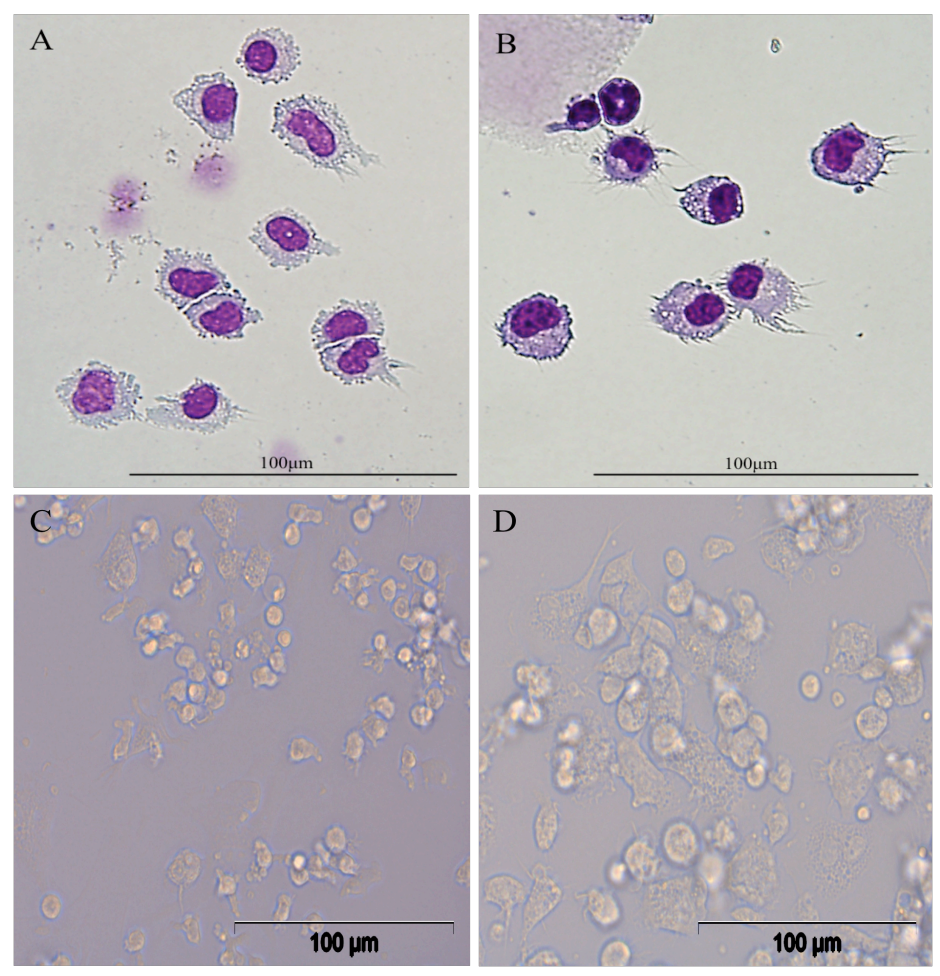

\section{Figure 3.3: Morphology of Flt3L-DCs}

BM cells from C57BL/6 mice were cultured in the presence of Flt3L for 9 days or GMCSF/IL-4 for 7 days. DCs were harvested on the final day of cell culture and centrifuged (300rpm, 8min) onto microscope slides (20,000 cells/slide). Flt3L (A) and GMCSF/IL-4 (B) DCs were stained using a Diff-Quik staining set and analysed under a microscope. Alternatively, Flt3L (C) and GMCSF/IL-4 (D) DCs were photographed directly in culture plates on the final day of cell culture. Original magnification for (A) and (B) at 50x and (C) and (D) at 20x. Scale bars represent $100 \mu \mathrm{m}$. Results are from one of two independent experiments. 
Morphological analysis does not easily distinguish the Flt3L-DC subsets that are generated in culture. Therefore, characterisation was further extended by phenotypic analysis using immunofluoresence staining and flow cytometry.

\subsubsection{Phenotypic characterisation of Flt3L-DCs by immunofluorescence staining and flow cytometry}

As reported in the literature, the culture of BM cells with Flt3L gives rise to three DC subsets, each characterised by differential expression of surface markers. These consist of pDCs, which express the marker B220 and two B220 negative cDCs subsets identified as $\mathrm{CD} 11 \mathrm{~b}^{\text {high }} \mathrm{CD} 24^{\text {low }}$ and $\mathrm{CD} 11 \mathrm{~b}^{\text {low }} \mathrm{CD} 24^{\text {high }}(214,225)$. All three subsets commonly share expression of the marker CD11c, which is highly expressed in DCs and is used to distinguish them from other cell types .

The phenotype of Flt3L-DCs was first characterised by immunofluorescence staining. Precursor cells derived from the BM of C57BL/6 mice were cultured in the presence of Flt3L for 9 days. DCs were harvested on day 9 of cell culture, centrifuged onto microscope slides (20,000 cells) and stained for CD11c and B220 (Figure 3.4B, 3.4C). Cell nuclei were then counter-stained using DAPI (Figure 3.4A). Examination under a fluorescent microscope confirmed the presence of the subset phenotypically equivalent to pDCs, as demonstrated by the expression of CD11c and B220 (Figure 3.4B, 3.4C). However, it was observed that only a small proportion of the cells stained positive for B220. Noticeably, the pDC subset showed an eccentric nucleus (Figure 3.4D) and few dendrites when viewed by phase contrast (Figure 3.4E). These features are in line with the established morphology of pDCs $(246,247)$. 

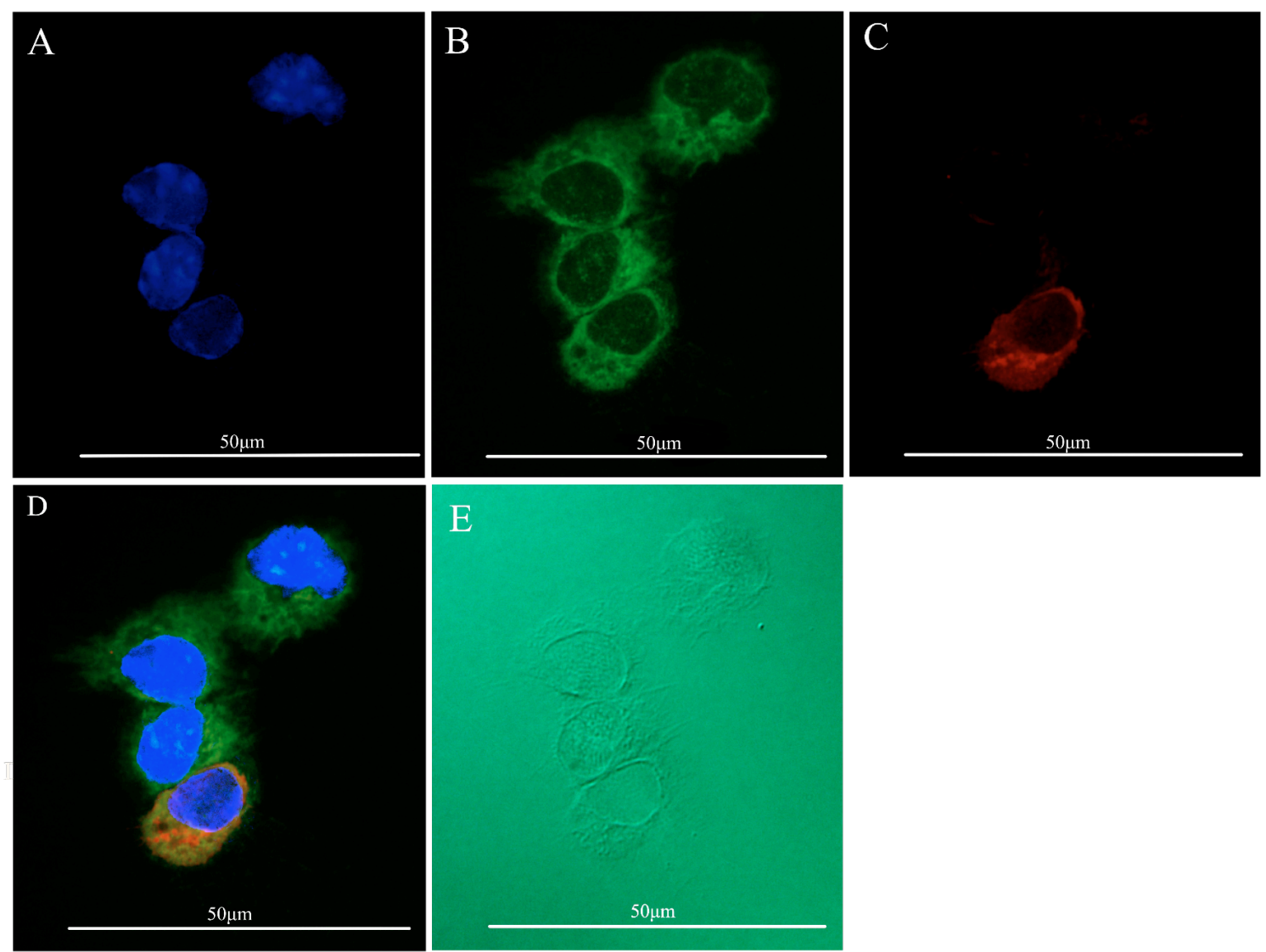

Figure 3.4: Detection of Flt3L-DC subsets by immunofluorescence staining.

BM cells extracted from the femur and tibia of C57BL/6 mice were cultured in the presence of Flt3L for 9 days to induce DC differentiation. On day 9, DCs were harvested and centrifuged (300rpm, 8 minutes) at room temperature onto microscope slides (20,000 cells per slide). Slides were air-dried and the cells were stained with the nucleic acid dye DAPI (A) and anti-CD11c-FITC mAb (B) to detect DC populations. pDCs were identified using B220-bio-SA-Alexa Fluor 555 (C). Panels D and E show the overlay of images from A to $\mathrm{C}$ and phase contrast, respectively. DCs were photographed using a fluorescence microscope under 100x magnification. Results are from one of two independent experiments that showed similar results. Scale bars represent $50 \mu \mathrm{m}$.

To identify and characterise the other subsets that are generated from Flt3L cultures, we further analysed their phenotype by flow cytometry. DCs derived from Flt3L cultures were harvested on day 9 and stained with mAbs for CD11c, B220, CD11b and CD24. Cells of interest were first identified based on analysis of cell size and internal complexity (FSC/SSC) (Figure 3.5). This initial gating strategy also accounted for the exclusion of 
dead cells, which could not be detected by a viability dye as the flow cytometer used for this experiment was limited to only four fluorophores per sample. DCs were identified by expression of $\mathrm{CD} 11 \mathrm{c}$ and subsequently analysed for $\mathrm{pDC}$ and $\mathrm{cDC}$ subsets. Flow cytometric analysis of Flt3L cultures revealed the presence of three distinct DC subsets: $\mathrm{B} 220^{+} \mathrm{pDCs}$ and $\mathrm{B} 220^{-} \mathrm{cDCs}$ subdivided into those that are $\mathrm{CD} 11 \mathrm{~b}^{\text {high }} \mathrm{CD} 24^{\text {low }}$ and $\mathrm{CD} 11 b^{\text {low }} \mathrm{CD} 24^{\text {high }}$ cDCs (Figure 3.5). Therefore, this observation confirmed the pDC subset as detected by immunofluoresence staining, and is in line with published data with respect to phenotypic characteristics of Flt3L-DCs generated in vitro $(214,225)$. Hereafter, the two cDC subsets will be refered to $\mathrm{CD} 11 \mathrm{~b}^{\text {high }}\left(\mathrm{CD} 11 \mathrm{~b}^{\text {high }} \mathrm{CD} 24^{\text {low }}\right)$ and $\mathrm{CD} 24^{\text {high }}\left(\mathrm{CD} 11 \mathrm{~b}^{\text {low }} \mathrm{CD} 24^{\text {high }}\right) \mathrm{cDCs}$.
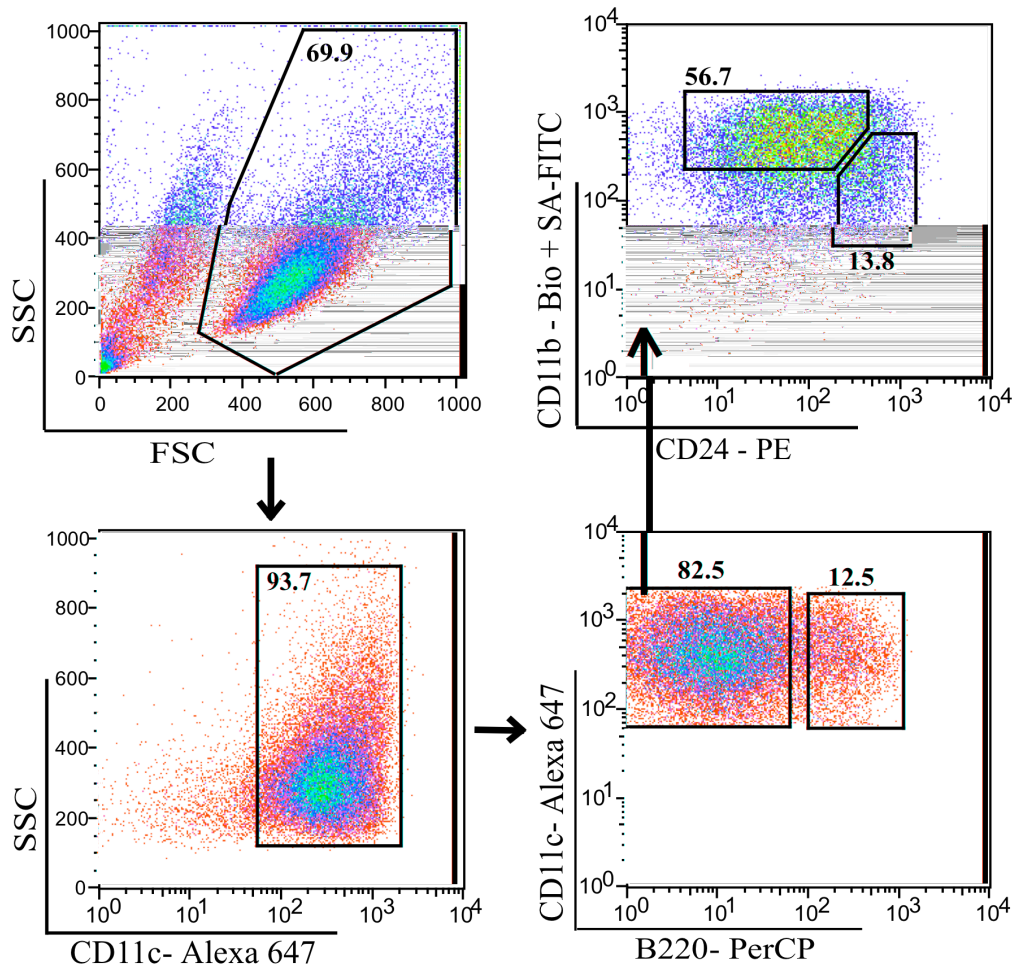

Figure 3.5: The culture of BM cells in Flt3L generates three DC subsets.

Flt3L-DCs were generated as described in Figure 3.4. Cells were harvested on day 9 and stained with mAbs for CD11c, B220, CD11b and CD24. Cells of interest were first identified based the on analysis of FSC and SSC properties. This was followed by gating of cells positive for CD11c and pDCs were identified by positive expression of B220. The cDC subset was identified based on negative expression of B220 and was further subdivided into subsets that were $\mathrm{CD} 11 \mathrm{~b}{ }^{\text {high }} \mathrm{CD} 24^{\text {low }}$ and $\mathrm{CD} 11 \mathrm{~b}{ }^{\text {low }} \mathrm{CD} 24^{\text {high }}$. Numbers indicate percentages of cells within the respective gating regions. Results are from one of several experiments. 


\subsubsection{Flt3L-DC subsets respond to stimulation with TLR agonists}

DC maturation is important for the overall quality of the anti-tumour immune response (248). It was therefore relevant to investigate the maturational capability of Flt3L-DCs and identify which TLR agonists can trigger effective maturation.

DCs derived from Flt3L cultures were stimulated with various TLR agonists on day 9 and incubated for 24 hours. The following day, cells were harvested and the expression of costimulatory molecules on pDCs and cDCs was determined by flow cytometry. The two cDC subsets could not be analysed individually, but only as a whole population, as the flow cytometers used for this experiment could only detect four fluorophores per sample. Concentrations of TLR agonists used to induce Flt3L-DC activation were selected based on those reported in the literature $(214,234)$. Flt3L-DC subsets were identified using the gating strategy shown in Figure 3.5.

Although we could not detect any statistical significance within our results due to a small sample size, we observed the following trends in our data. Compared to the unstimulated control, cDCs responded to stimulation with all the TLR agonists and there were no considerable differences among the agonists at inducing the up-regulation of costimulatory molecules and MHC II (Figure 3.6A). Furthermore, cDCs were observed to have a higher maturation status compared to pDCs, as evidenced by higher expression levels of activation markers. In contrast to cDCs, pDCs preferentially responded to stimulation with $\mathrm{CpG}$, as evidenced by greater up-regulation of CD80, CD86 and MHC II (Figure 3.6B). However, CD40 up-regulation on pDCs was induced to the greatest extent by the TLR agonist Poly I:C. LPS and Pam3Cys were the least effective agonists on pDCs overall. In summary, Flt3L-DC subsets responded differentially to stimulation with TLR agonists. While cDC up-regulated expression of activation markers in response to all TLR agonists, pDCs mainly favoured $\mathrm{CpG}$.

As Flt3L-DCs were only stimulated with TLR agonists at concentrations selected from the literature, all agonists were subsequently titrated three fold higher and lower to confirm that concentrations initially selected were optimal (Appendix 3). The results were variable, 
whereby some activation markers increased or decreased on cDCs and pDCs in response to stimulation with higher concentrations of the selected agonists. However, overall the results showed that the concentration initially selected induced up-regulation of most activation markers. Thus, the concentrations originally used remained the same for subsequent experiments. 
A) cDCs
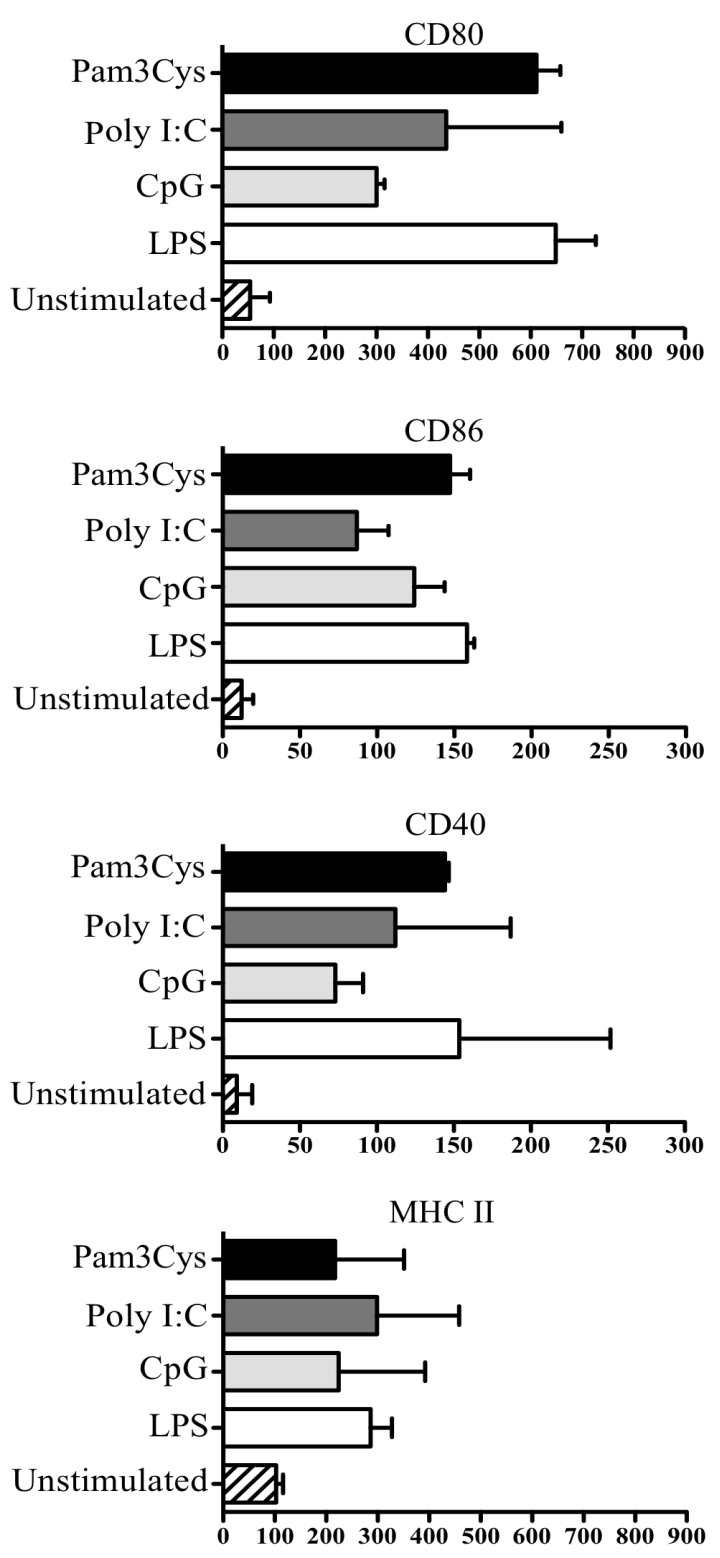

B) $\mathrm{pDCs}$

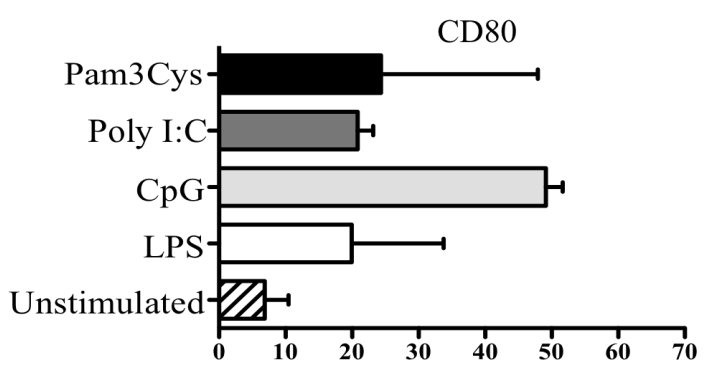

CD86

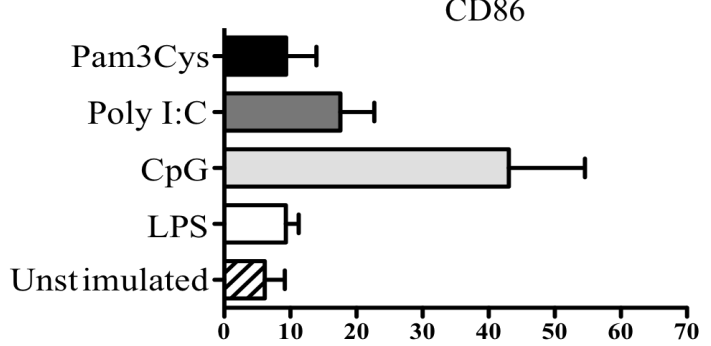

CD40

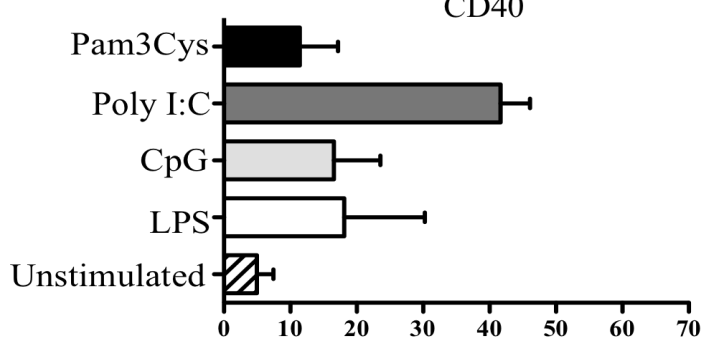

MHC II

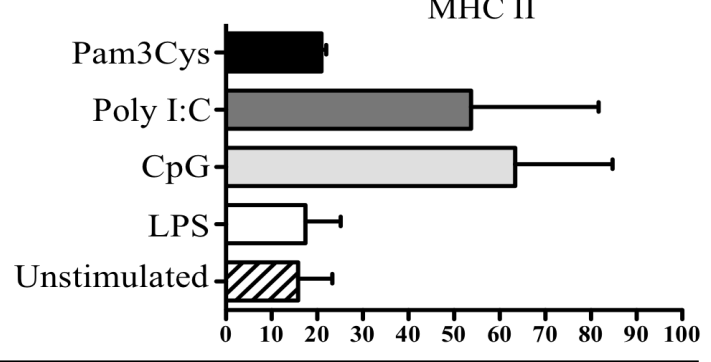

MFI

\section{Figure 3.6: FIt3L-DC subsets display a trend to activation upon TLR stimulation.}

Flt3L-DCs were generated as described in Figure 3.4. On day 9 of cell culture, Flt3L-cDCs were stimulated for 24 hours with the following TLR agonists: LPS (100ng/mL), CpG $(2 \mu \mathrm{g} / \mathrm{mL})$, Poly I:C (50 $\mu \mathrm{g} / \mathrm{mL})$ and Pam3Cys $(1 \mu \mathrm{g} / \mathrm{mL})$ or left untreated as a control. Cells were harvested the following day and stained with varying combinations of $\mathrm{mAbs}$ for $\mathrm{CD} 11 \mathrm{c}, \mathrm{B} 220, \mathrm{CD} 80, \mathrm{CD} 86, \mathrm{CD} 40$ and MHC II. Expressions of costimulatory molecules were determined in $\mathrm{cDCs}(\mathrm{A})$ and $\mathrm{pDCs}(\mathrm{B})$. Bar graph values represent mean \pm S.D. Results are cumulated data from two independent experiments. Statistical significance was tested using the Kruskal-Wallis with Dunn's multiple comparisons test. No significance was detected. 


\subsubsection{Depletion of pDCs does not prevent the response of $\mathrm{cDCs}$ to stimulation with TLR agonists}

As shown in Figure 3.6, cDCs exhibited a higher maturation status compared to pDCs upon stimulation with TLR agonists. This observation raised the question as to whether the maturational response was due to the interaction between $\mathrm{cDC}$ and $\mathrm{pDCs}$. It is possible that following maturation, $\mathrm{pDCs}$ can produce type 1 IFN that may participate in the activation of cDCs (234). Therefore, we sought to determine if depletion of pDCs affects the maturational capacity of cDCs.

Flt3L-DCs were harvested on day 9 of culture and depleted of $\mathrm{B} 220^{+} \mathrm{pDCs}$ by magnetic bead separation (Chapter 2, (2.4.3.4)). cDCs were subsequently re-plated back into culture and stimulated with TLR agonists for 24 hours. Flt3L-DCs not intended for depletion, but for use as a comparison were also harvested and treated in the same manner as the depleted population. This was to account for the possibility of spontaneous activation that may arise due to handling of the cells.

Results showed that pDC depletion does not prevent the response of cDCs to TLR stimulation. However, some differences were observed in the up-regulation CD80 and MHC II, but this was not statistically significant (Figure 3.7A). Therefore, these results indicated that cDCs could maintain their maturational capacity in the absence of pDCs. However, given that the method, which we used to deplete pDCs only resulted in $70-76 \%$ depletion, it cannot be excluded that the remaining population of pDCs may still directly impact on cDC maturation (Appendix 4A). Therefore, to improve the purity of the cDC population, Flt3L cultures were positively sorted for cDCs by FACS, which resulted in 99\% purity of cDCs (Appendix 4B). Flt3L-DCs that were not sorted for cDCs, but used for comparative purposes were treated in the same manner. In comparison to cDCs magnetically depleted of pDCs (Figure 3.7A), analysis of sorted cDCs revealed similar patterns of CD80 and CD86 expression among the agonist treated groups (Figure 3.7B). Results for CD40 and MHC II expression could not be obtained due to an antibody labelling error. In summary, it does not appear that depletion of pDCs hinders the ability of cDCs to up-regulate co-stimulatory molecules following TLR stimulation. 
A)

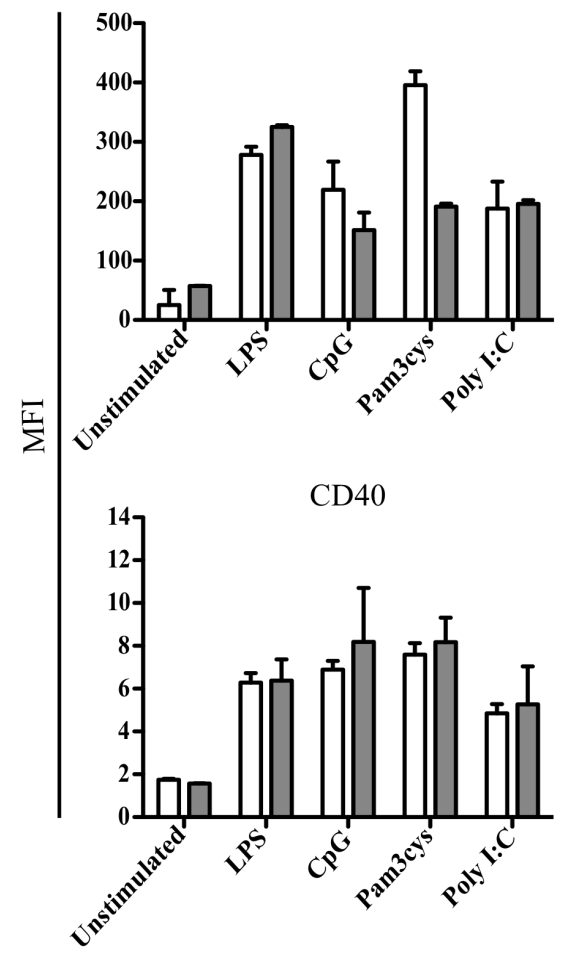

CD86

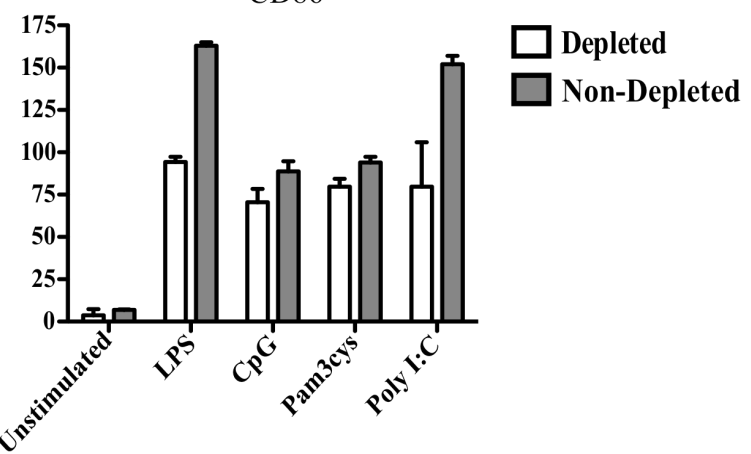

MHC II

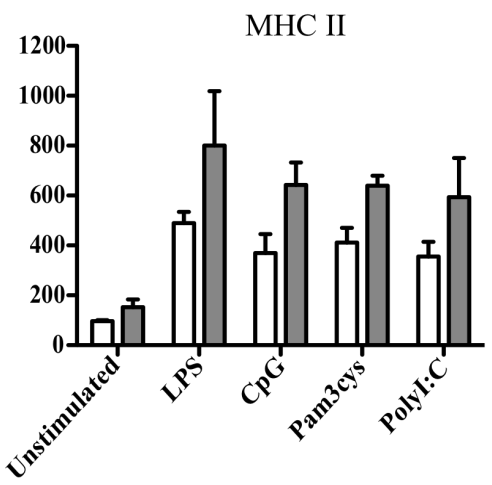

B)
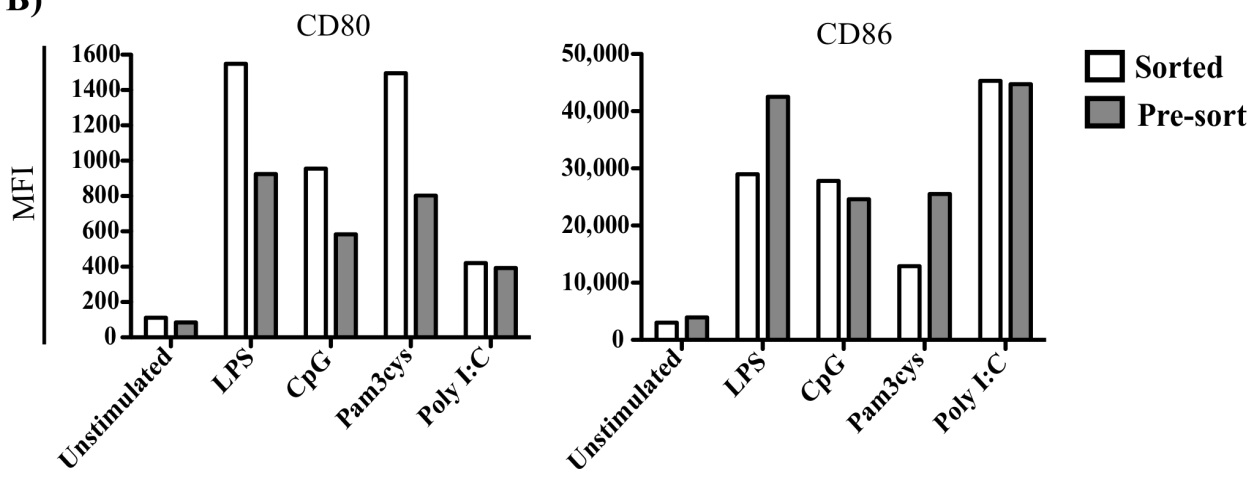

Figure 3.7: cDCs are capable of maturation in the absence of pDCs. Flt3L-DCs were generated as described in Figure 3.4. On day 9, Flt3L-DC cultures were depleted of pDCs by magnetic separation (A) or CDCs were sorted by FACS (B). Flt3L-DCs not intended for depletion were also harvested on day 9 and treated in the same manner with respect to each method of depletion (A and B). Following depletion or sort, cDCs were placed back into culture and stimulated for 24 hours with the following TLR agonists: LPS (100ng/mL), CpG $(2 \mu \mathrm{g} / \mathrm{mL})$, Poly I:C $(50 \mu \mathrm{g} / \mathrm{mL})$ and Pam3Cys $(1 \mu \mathrm{g} / \mathrm{mL})$. The following day, cDCs were harvested, stained with various combinations of mAbs for CD11c, B220, CD80, CD86, CD40 and MHC II, and analysed by flow cytometry. Bar graph values represent mean \pm range. (A) shows averages of duplicate samples from one experiment only. (B) shows data from only one experiment. 


\subsubsection{Stimulation with combined TLR agonists improves the maturation of Flt3L-DCs}

It was established that Flt3L-DCs could mature upon stimulation with TLR agonists, as characterised by the up-regulation of co-stimulatory molecules and MHC II (Figure 3.6). To extend on this finding, we next investigated the effect of combined TLR ligation on Flt3L-DCs, which has been reported to enhance the maturation of GM-CSF DCs (235, 236).

Flt3L-DCs were generated as described in Figure 3.4. On day 9 of cell culture, Flt3L-DCs were stimulated with all the possible combinations of TLR agonists at the predetermined concentrations or left unstimulated as a control. Cells were incubated for 24 hours and harvested the following day to determine the level of co-stimulatory molecules and MHC II expression on cDCs and pDCs by flow cytometry.

In comparison to single TLR agonists, selected TLR agonist combinations were observed to further increase expression of both CD40 and CD86 on cDCs and pDCs. In particular, this was predominantly seen with the combinations of LPS/Poly I:C, LPS/Pam3Cys and Pam3Cys/Poly I:C (Figure 3.8A and 3.8B), all of which induce the triggering of both MyD88-dependent and-independent signalling (Figure 3.1). It was also noteworthy that LPS/CpG was effective at improving CD40 up-regulation on cDCs, and both CD40 and CD86 on pDCs. With respect to MHC II expression on cDCs, we did not observe any improvement in the up-regulation of this activation marker following stimulation with the various agonist combinations. In contrast, we found that the combinations of $\mathrm{LPS} / \mathrm{CpG}$, CpG/Poly I:C and Pam3Cys/Poly I:C increased expression of MHC II on pDCs. However, the effects on MHC II up-regulation from these combinations were very weak, when compared to the respective individual agonists.

Overall, we found that combining TLR agonists could provide a boost in the up-regulation of activation markers on Flt3L-DC subsets. More specifically, combined TLR stimulation had its most pronounced effect on enhancing the up-regulation of CD40 and CD86 on cDCs and pDCs. 
Figure 3.8: Combined TLR stimulation enhances Flt3L-DC maturation. Flt3L-DCs were generated as described in Figure 3.4. On day 9 of cell culture, activation was induced by stimulation with the individual or combined TLR agonists at the following concentrations: LPS (100ng/mL), CpG $(2 \mu \mathrm{g} / \mathrm{mL})$, Poly I:C $(50 \mu \mathrm{g} / \mathrm{mL})$ and Pam3Cys $(1 \mu \mathrm{g} / \mathrm{mL})$. After 24 hours of stimulation, Flt3L-DCs were harvested and stained with varying combinations of mAbs for CD11c, B220, CD40, CD80 and MHC II. Expressions of co-stimulatory molecules and MHC II were determined in cDCs (A) and pDCs (B). Bar graph values represent mean \pm S.D. $*=0.01<P<0.05$, as determined by the Kruskal-Wallis with Dunn's multiple comparison's test. Results show averages of triplicate samples from one out of two experiments with similar results. 
A) cDCs
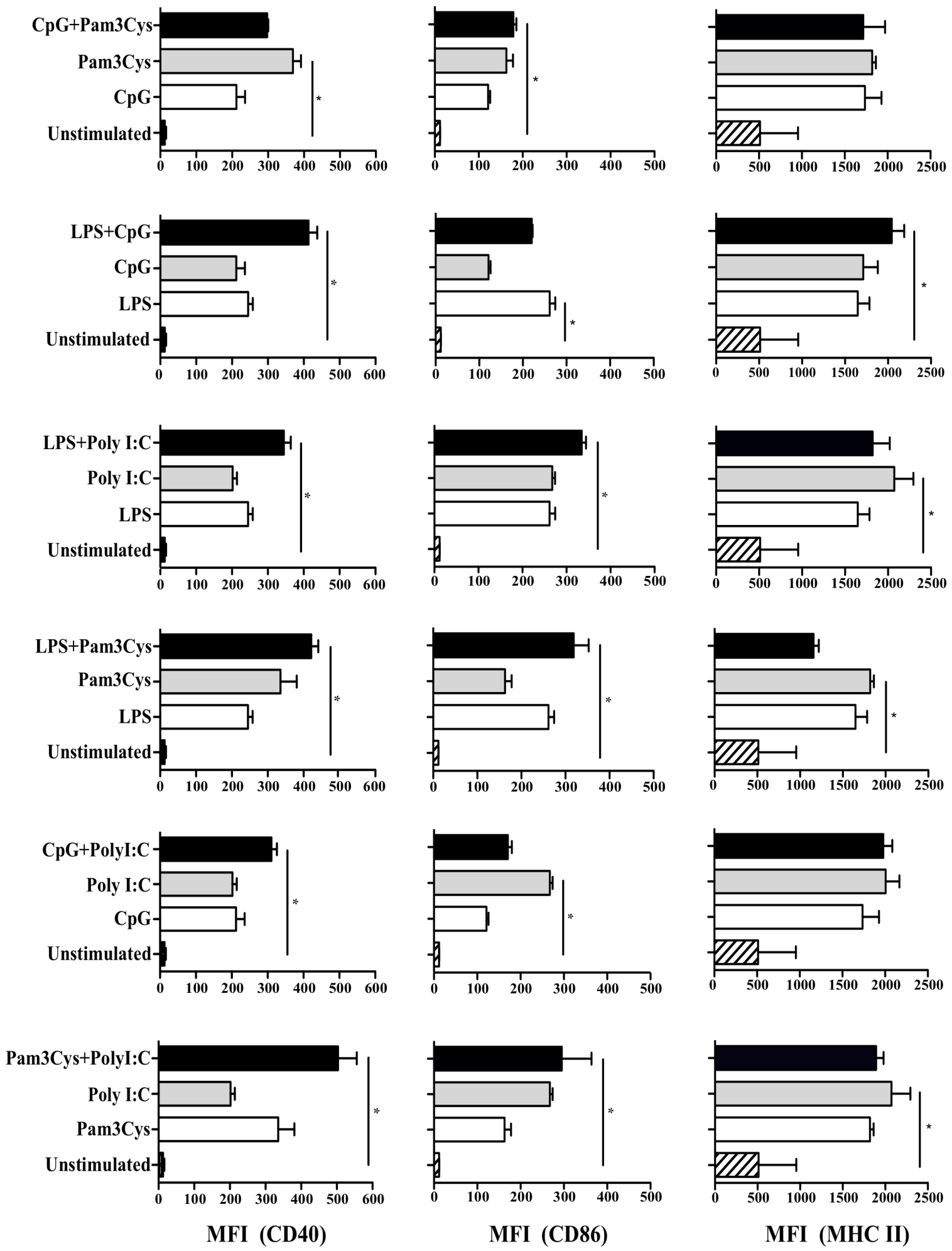
B) pDCs
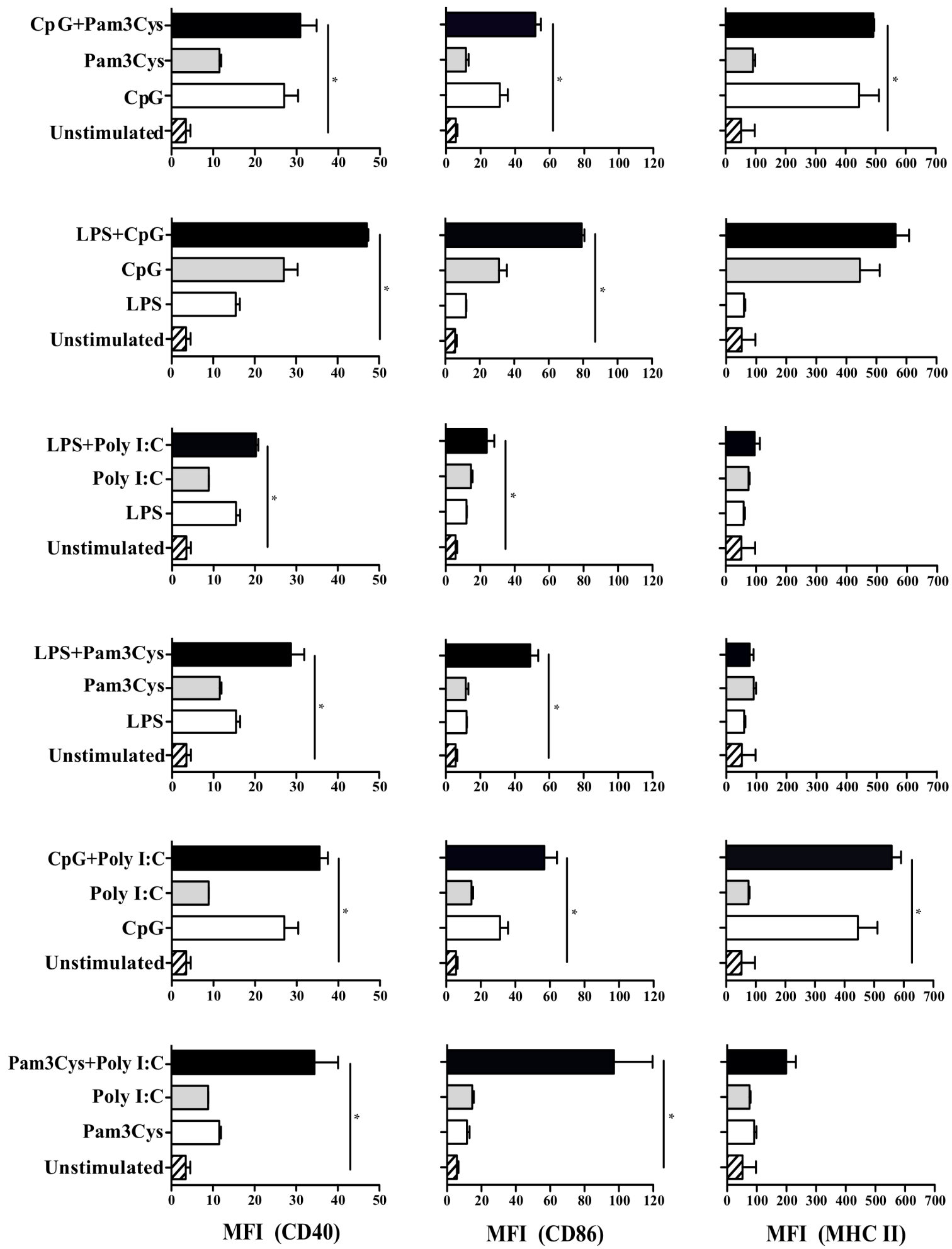
Cellular viability was also determined following stimulation with TLR agonist combinations to determine whether receiving more than one stimulus is toxic to the cells. Viability of Flt3L-DCs was analysed as a whole population and it was found that treatment with the combined TLR agonists did not induce a significant decrease in the percentage of live cells in comparison to stimulation with the respective individual agonists (Figure 3.9). Thus, stimulation with more than one TLR agonist does not significantly impact on Flt3LDC viability.

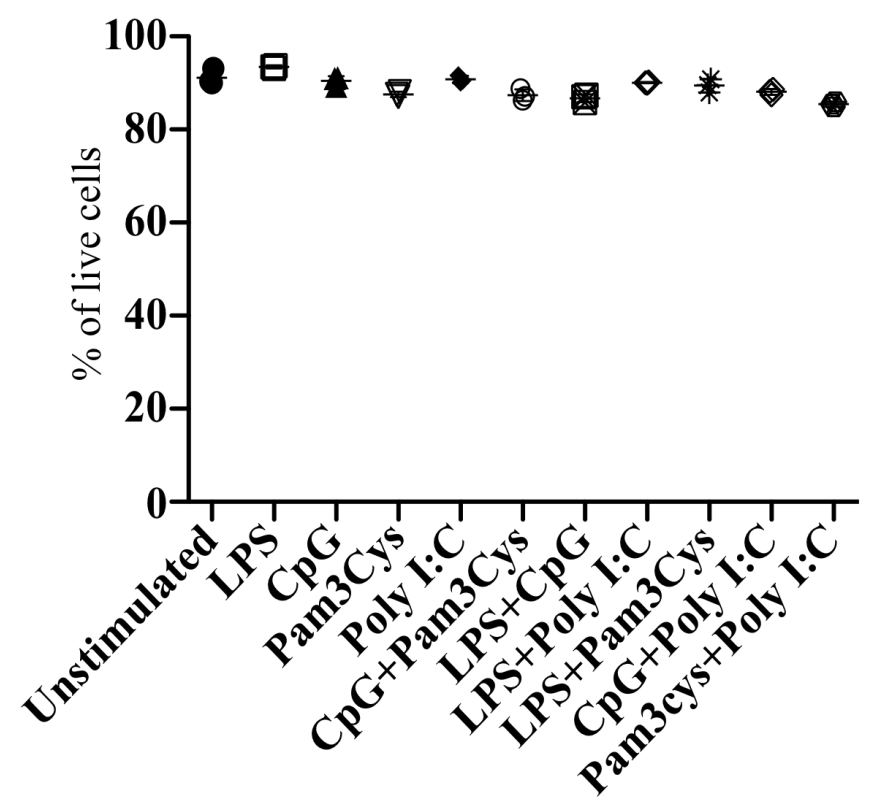

Figure 3.9: Stimulating Flt3L-DCs with TLR agonist combinations does not reduce cell viability. Flt3L-DCs were generated as described in Figure 3.4. On day 9 of cell culture, activation was induced by stimulation with TLR agonists either alone or in combination with each other at the following concentrations: LPS (100ng/mL), CpG $(2 \mu \mathrm{g} / \mathrm{mL})$, Poly I:C $(50 \mu \mathrm{g} / \mathrm{mL})$ and Pam3Cys $(1 \mu \mathrm{g} / \mathrm{mL})$. After 24 hours of stimulation, Flt3L-DCs were harvested, stained with the viability dye PI, and analysed by flow cytometry. Cells that were CD11 $\mathrm{c}^{+}$and excluded PI were identified as live cells. Graph values represent mean \pm S.D. Results show averages of triplicate samples from one out of two experiments. Statistical significance was tested using the Kruskal-Wallis with Dunn's multiple comparisons test. No significance was detected. 


\subsubsection{Low concentrations of 2-DG inhibit the maturation of GMCSF/IL- 4 DCs but not Flt3L-cDCs}

Results presented within this chapter have phenotypically characterised Flt3L-DCs before and following stimulation with various TLR agonists. To further extend our characterisation, we investigated the impact of inhibiting glucose metabolism on the maturation of Flt3L-DCs. Krawczyk et al demonstrated the dependence on glucose metabolism of LPS stimulated GM-CSF DCs by treating cells with 2-Dexoy-D-glucose (2DG), a glucose analogue that acts as competitive inhibitor of glucose metabolism (245). Under normal conditions of glycolysis, the first and rate-limiting step is the conversion of glucose to glucose-6-phosphate by the enzyme hexokinase (Figure 3.2) (249). When cells are treated with 2-DG, this is used instead of glucose and is converted by hexokinase into phoshorylated 2-DG. The phosphorylated 2-DG cannot be further metabolised and becomes trapped within the cell. As a consequence, intracellular ATP is depleted (250). Krawczyk et al showed that stimulation in the presence of 2-DG resulted in decreased expression of CD40, CD86 and MHC II expression compared to 2-DG untreated controls. We therefore sought to confirm the findings of Krawczyk et al in our GMCSF/IL-4 culture condition, and to establish if the same results apply to Flt3L-DCs.

Krawczyk et al have shown that activated GM-CSF DCs subjected to glucose limitation for 24 hours, resulted in a reduction of DC survival (Section 3.1.2). Thus, stimulating both Flt3L-cDCs or GMCSF/IL-4 DCs for 24 hours in the presence of 2-DG was likely to impact on cell survival, and activation markers would not be able to be properly examined. Due to this, we first determined the LPS concentration that could mature GMCSF/IL-4 and Flt3L-DCs after 6 hours of stimulation.

BM cells were cultured in the presence of Flt3L for 9 days or with GMCSF/IL-4 for 7 days. Due to a shorter period of cell culture, GMCSF/IL-4 DCs were set up 3 days following Flt3L-DC cultures, allowing for stimulation with LPS and subsequent analysis to occur on the same day. Flt3L-DCs subsets were identified as shown in Figure 3.5 and GMCSF/IL-4 DCs were identified by expression of the marker CD11c ${ }^{+}$. Analysis of cell viability in both DC populations was determined by exclusion of PI (Appendix 5). Only 
the cDC subset was chosen specifically for comparison with GMCSF/IL-4 DCs for this and subsequent experiments, as it was established that LPS was not an effective agonist at inducing pDC maturation (Figure 3.5). Hereafter, the $\mathrm{cDC}$ subset will be described as Flt3L-cDCs, with respect to experiments evaluating glycolytic metabolism in Flt3L and GMCSF/IL-4 DCs.

Given that both DC populations were only stimulated for 6 hours, we selected LPS concentrations that could induce maximal expression of activation markers without affecting cell viability. We observed that $500 \mathrm{ng} / \mathrm{mL}$ of LPS did not impact on the viability of GMCSF/IL-4 DCs and induced the greatest level of CD80 and CD40 expression, compared to all other concentrations of LPS (Figure 3.10A, 3.10C). Therefore, $500 \mathrm{ng} / \mathrm{mL}$ was considered an optimal concentration, and was thus selected for inducing the activation of GMCSF/IL-4 DCs in subsequent experiments. Conversely, Flt3L-cDCs did not favour higher concentration of LPS, as we observed a reduction of MHC II expression, in a dosedependent manner (Figure 3.10B). Therefore, as we observed maximal expression of MHC II following stimulation with $100 \mathrm{ng} / \mathrm{mL}$ of LPS, and similar viability compared to untreated controls, we selected this concentration to stimulate Flt3L-cDCs (Figure 3.10B, 3.10C). 
A) GMCSF/IL-4 DCs
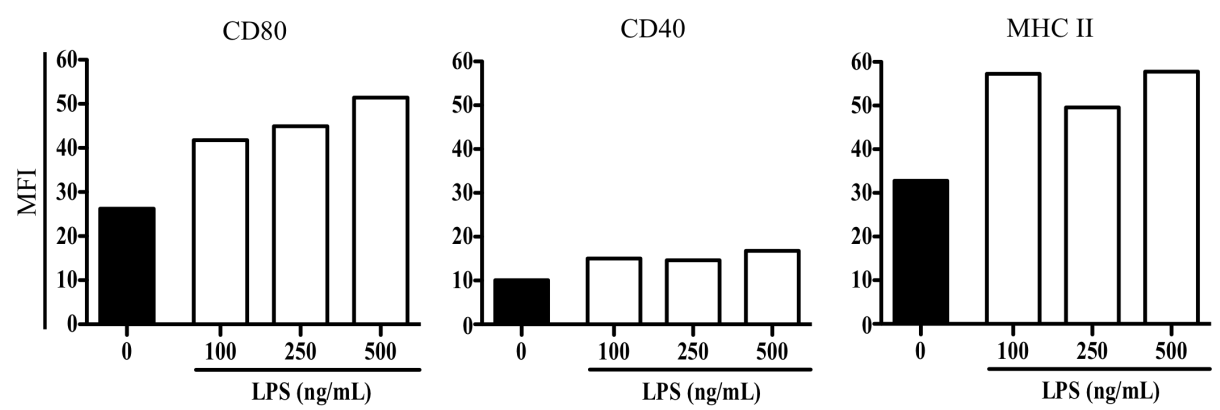

B) FIt3L-cDCs
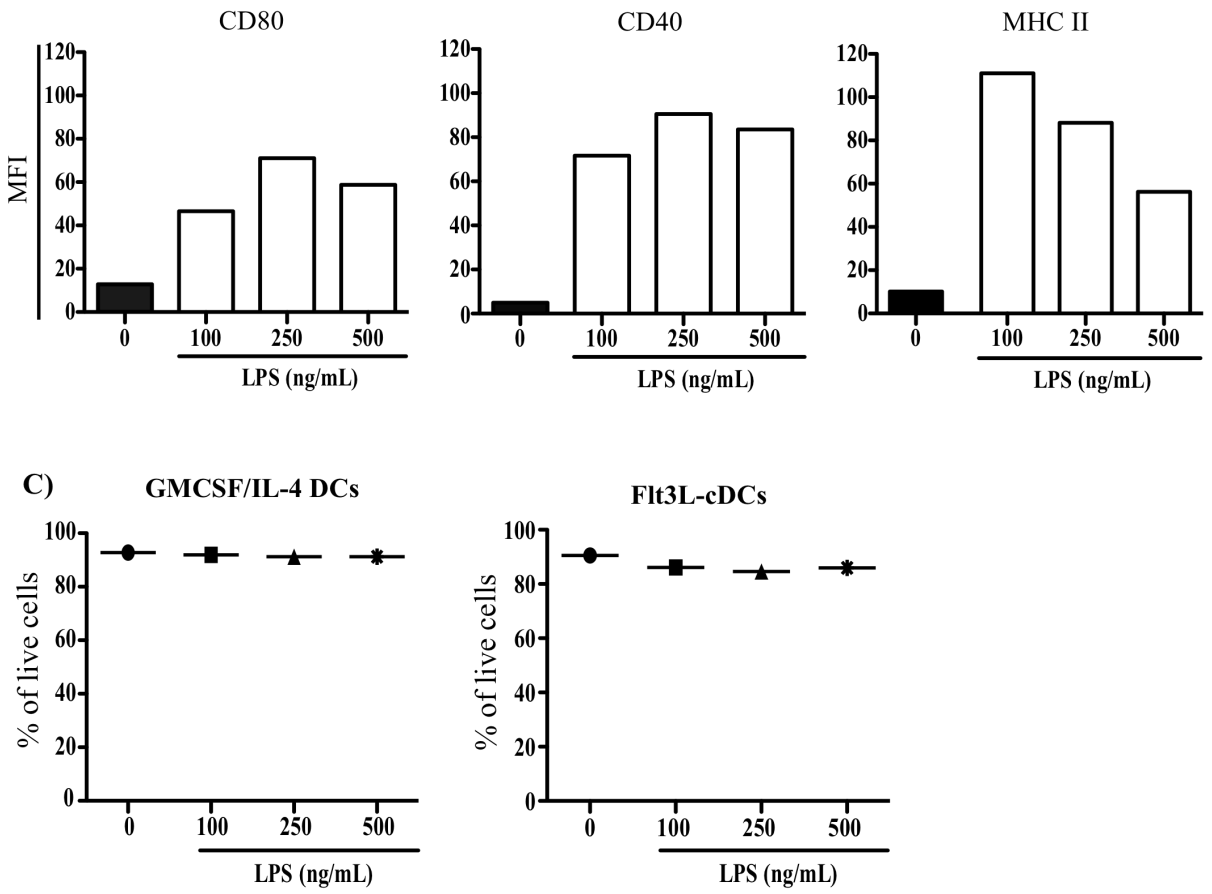

Figure 3.10: Treatment with LPS induces the maturation of Flt3L and GMCSF/IL-4

DCs following a 6 hour stimulation. BM cells were cultured with Flt3L for 9 days or GMCSF/IL-4 for 7 days to induce DC differentiation. Stimulation with LPS at concentrations of $100 \mathrm{ng} / \mathrm{mL}, 250 \mathrm{ng} / \mathrm{mL}$ and $500 \mathrm{ng} / \mathrm{mL}$ was performed on day 10 or 7 for Flt3L and GMCSF/IL-4 DCs respectively. Following 6 hours of incubation, cells were harvested and analysed by flow cytometry to detect CD40, CD80 and MHC II expression in GMCSF/IL-4 (A) and Flt3L-cDCs (B). (C) Cell viability following stimulation with selected LPS concentrations was assessed. Results of one experiment only are shown.

To determine if glycolytic metabolism is essential for the maturation of Flt3L-cDCs, both Flt3L and GMCSF/IL-4 DCs cultures were generated purposely to allow for stimulation 

with TLR agonists

with LPS and treatment with 2-DG to occur on the same day. Both DC cultures were treated with LPS at the predetermined concentrations (Figure 3.10), followed by treatment with titrated doses of 2-DG at either $6.25 \mathrm{mM}, 12.5 \mathrm{mM}$ or $25 \mathrm{mM}$. DCs were incubated for 6 hours, harvested and analysed by flow cvtometry to determine the level of activation markers. The highest dose of 2-DG $(25 \mathrm{mM})$ was equivalent to the total concentration of glucose in the culture media.

Treatment with 2-DG in parallel to stimulation with LPS impaired the ability of GMCSF/IL-4 DCs to up-regulate activation markers (Figure 3.11A). Despite the concentration of 2-DG used, the level of activation markers remained at least 2 fold lower compared to LPS treated only controls (Figure 3.11C). Furthermore, LPS-stimulated GMCSF/IL-4 DCs treated with various concentrations of 2-DG maintained viability similar to those of LPS only controls. Therefore, this indicated that inhibition of activation markers was not due to decreased DC survival (Figure 3.11E).

Upon examination of Flt3L-cDCs, we found that expression of activation markers was differentially affected by treatment with 2-DG. Flt3L-cDCs stimulated with LPS and treated with a high concentration $(25 \mathrm{mM})$ of 2-DG exhibited reduced viability and impairment in the up-regulation of the activation markers CD40 and MHC II. Conversely, Flt3L-cDCs treated with intermediate or low concentrations of 2-DG $(12 \mathrm{mM}$ or $6.25 \mathrm{mM})$ maintained viability, but only those treated with the lowest dose of 2-DG were able upregulate the activation markers CD80 and MHC II at levels comparable to the LPS only stimulated control (Figure 3.11B, 3.11E). CD40 expression on LPS-stimulated Flt3LcDCs remained considerably low across all 3 concentrations of 2-DG used, but is nonetheless higher when compared to untreated controls (Figure 3.11B, 3.11D). It was also observed that Flt3L-cDCs treated with 2-DG only, expressed decreased baseline expression of activation markers in a dose-dependent manner (Figure 3.11B). This reduction in expression of activation markers also correlated with a decrease in the viability of Flt3L-cDCs (Figure 3.11E). In summary, both GMCSF/IL-4 DCs and Flt3LcDCs displayed differential responses to inhibition of glycolytic metabolism, in both the non-activated and activated state. 
Figure 3.11: Low concentrations of 2-DG have limited effects on the maturation of FIt3L-cDCs induced by LPS, but strongly affect the maturation of GMCSF/IL-4 DCs.

BM cells were cultured with Flt3L for 9 days or GMCSF/IL-4 for 7 days. LPS stimulation occurred on day 10 for Flt3L-DCs and day 7 for GMCSF/IL-4 DCs at a final concentration of $100 \mathrm{ng} / \mathrm{mL}$ and $500 \mathrm{ng} / \mathrm{mL}$, respectively. The competitive glucose inhibitor 2-DG was added at $25 \mathrm{mM}, 12.25 \mathrm{mM}$ or $6.25 \mathrm{mM}$, as indicated by the triangles. Following 6 hours of incubation, Flt3L-DCs were harvested and stained with various combinations of mAbs for CD11c, B220, CD40, CD80 and MHC II to determine expression. GMCSF/IL-4 DCs were stained with various combinations of mAbs for CD11c, CD40, CD80 and MHC II. Both DC populations were subsequently stained with the viability dye DAPI and cells were analysed by flow cytometry. Maturation of and GMCSF/IL-4 DCs (A) an Flt3L-cDCs (B) was determined after stimulation with LPS in the presence of varying concentrations of 2-DG and with the indicated controls. (C) and (D) show normalised marker expression in LPS and 2-DG treated groups, calculated as the percent of expression in the LPS-untreated group exposed to the same dose of 2-DG (data not shown within C and D). (E) Analysis of cell viability was determined in GMCSF/IL-4 DCs and Flt3L-cDCs in the indicated groups. Bar graph values represent mean \pm S.D. Results show averages of triplicate samples from one experiment only. 
A) GMCSF/IL-4 DCs
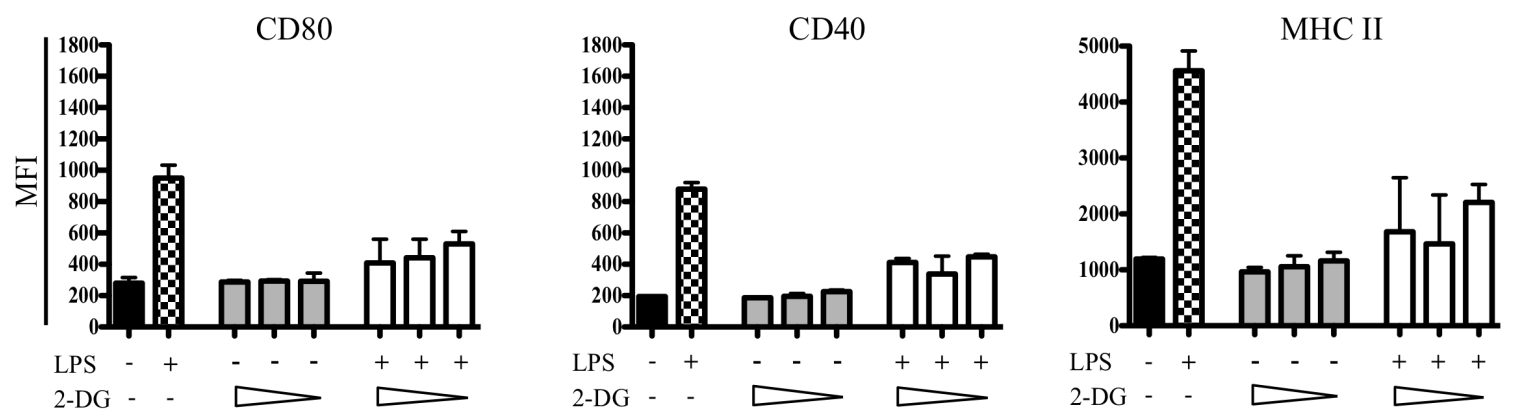

B) Flt3L-cDCs
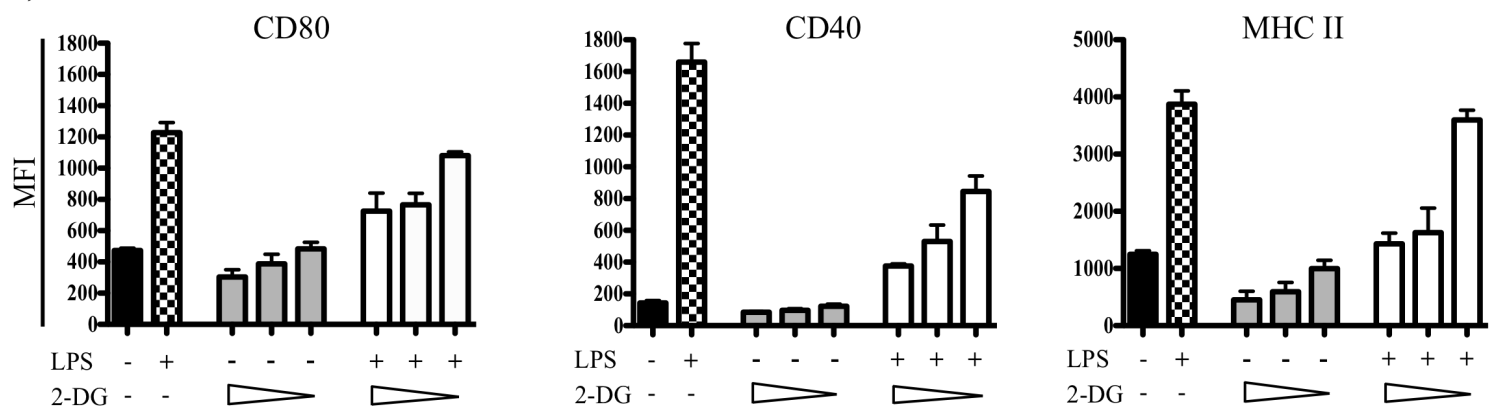

C) GMCSF/IL-4 DCs - Normalised marker expression
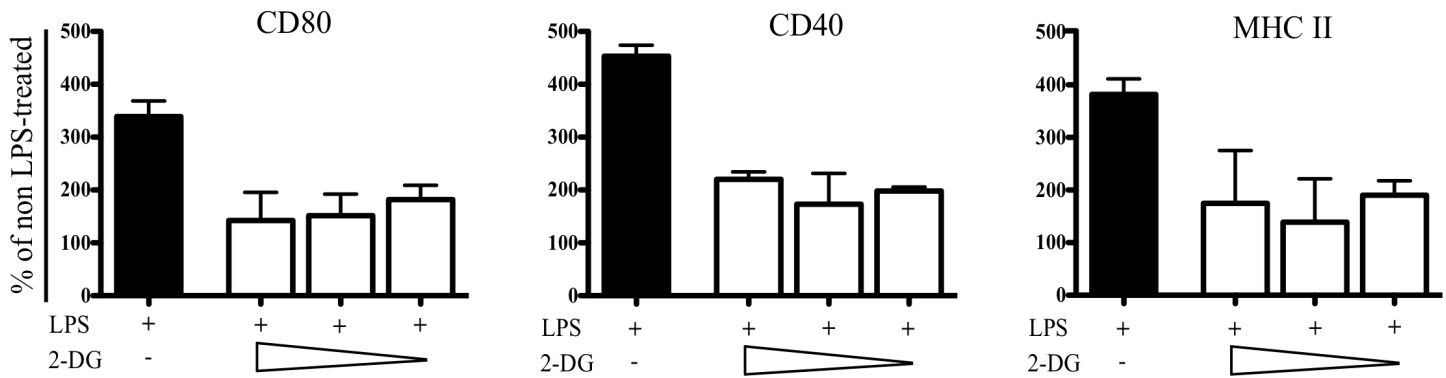

D) Flt3L-cDCs - Normalised marker expression
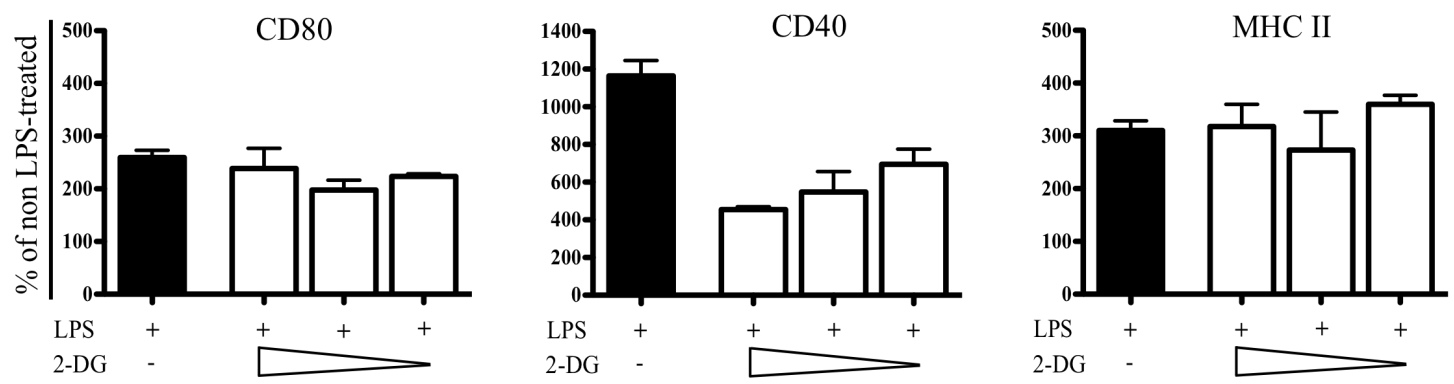
Chapter 3: Characterisation of Flt3L-DCs and evaluation of their response to stimulation

81 with TLR agonists

E)

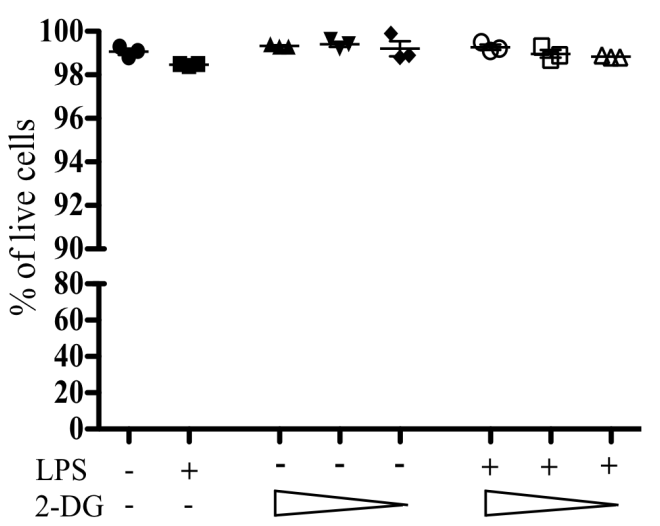

Flt3L-cDCs

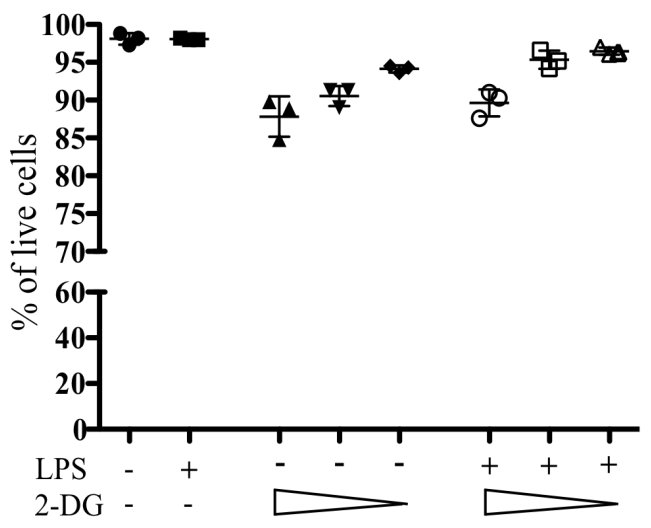




\subsection{Discussion}

\subsubsection{Phenotypic and morphological characteristics of Flt3L-DCs}

The results presented within this chapter describe the morphological and phenotypic characteristics of Flt3L-DCs generated within our laboratory. It was shown that the culture of BM cells in the presence of Flt3L generated three distinct DC subsets (Figure 3.4, 3.5). The first subset consisted of pDCs and was phenotypically characterised as CD $11 \mathrm{c}^{+} \mathrm{B} 220^{+}$. Flt3L also gave rise to cDCs, which were divided into two subsets that were CD11 $b^{\text {high }}$ and $\mathrm{CD} 24^{\text {high }}$. The $\mathrm{pDC}, \mathrm{CD} 11 \mathrm{~b}{ }^{\text {high }}$ and $\mathrm{CD} 24^{\text {high }}$ subsets identified within this study are similar to those described by Naik et al, who also characterised these subsets to be the in vivo counterparts of mouse splenic pDCs, $\mathrm{CD}^{+}$and $\mathrm{CD}^{-} \mathrm{cDCs}$, respectively (225). Analysis of cell morphology by Diff-Quik staining did not show a clear difference in size between Flt3L and GMCSF/IL-4 DCs as reported by others $(214,231)$. This result could possibly be due to the centrifugal force of spinning the DCs onto the slide, which may have modified the appearence of the cells. Direct imaging of DCs in culture, however, did show that Flt3L-DCs were smaller in size in comparison to GMCSF/IL-4 DCs (Figure 3.3). Collectively, these results indicated that Flt3L-DCs generated within our cultures are in line with those described in published reports, with respect to the surface phenotype and cell morphology $(214,225,231)$.

Although there are similarities in phenotype, our data show a difference in the relative proportions of the $\mathrm{CD} 11 \mathrm{~b}{ }^{\text {high }}$ and $\mathrm{CD} 24^{\text {high }} \mathrm{cDCs}$ (Figure 3.5 ). The literature describes the $\mathrm{CD} 24^{\text {high }}$ subset as the larger propotion of the $\mathrm{cDC}$ population $(7,10)$. It is shown within this chapter that cDCs consisted of larger percentage of CD11b $b^{\text {high }}$ and a smaller percentage that was $\mathrm{CD} 24^{\text {high }}$. The difference in percentages could be due to the concentration of Flt3L used within our culture method as it was observed that the proportion of $\mathrm{cDC}$ subsets generated changed with the concentration of Flt3L used. For instance, $1 \%$ of Flt3L generated $35 \% \mathrm{CD} 24^{\text {high }} \mathrm{cDCs}$, while $10 \%$ Flt3L generated $45 \%$ CD2 $4^{\text {high }}$ cDCs (Evelyn Hyde, Malaghan Institute of Medical Research, unpublished data). Therefore, this is an indication that the proportion of the cDC subsets generated in vitro, can be regulated by altering the concentration of Flt3L. 


\subsubsection{Response of Flt3L-DCs to stimulation with individual TLR agonists}

The literature has consistently shown that maturation of DCs is a critical parameter for the induction of immunity $(64,251)$. We investigated if Flt3L-DCs could mature and acquire an activated phenotype, following stimulation with various individual TLR agonists. To reiterate, the TLR agonists used within this study and their corresponding receptors were, Pam3Cys, Poly I:C, LPS and CpG, which are recognised by TLR2, 3, 4 and 9 respectively (Figure 3.1) (240).

In this chapter, we observed certain trends in the up-regulate activation markers on Flt3LDCs in response to stimulation with TLR agonists. Specifically, all TLR agonists were effective at inducing the maturation of cDCs, with no considerable differences seen between the stimuli. However, $\mathrm{CpG}$ was more effective at inducing $\mathrm{pDC}$ maturation, compared to all other agonists (Figure 3.6). One possible reason for this difference could be due to the expression of TLRs. It is well established that TLR expression is not uniform among the DC subsets $(107,252)$. Murine cDCs analysed as a whole population, have been shown to express all of the TLRs relevant to the agonists used within this study (233). Therefore, given that all receptors are present, this is likely to explain why cDCs were observed to respond to all TLR agonists. However, it is important to note that the $\mathrm{CD} 11 \mathrm{~b}^{\text {high }}$ and CD24 $4^{\text {high }} \mathrm{cDCs}$, have also been shown to differentially express the TLRs. Specifically, Naik et al showed that CD11b ${ }^{\text {high }}$ cDCs expressed high levels of TLR4, but TLR3 were not expressed at levels detectable by real-time PCR. Conversely, CD24 $4^{\text {high }}$ cDCs exhibited high TLR3 expression and lower expression of TLR4 (225). Thus, a different result is likely to be observed if the subsets were analysed separately.

In contrast to cDCs, studies have shown that murine pDCs highly express TLR9 and weakly express TLR2, 3 and $4(11,30)$. A higher expression of TLR9 may explain why CpG was the most effective agonist at inducing pDC maturation and LPS and Pam3Cys the least effective. With that said, it was interesting to find that instead of CpG, Poly I:C could induce greater up-regulation of CD40 on pDCs. Potentially, this response maybe 
due to the inflammatory cytokines that are released by cDCs, upon activation. It has been reported that inflammatory cytokines can induce the up-regulation of co-stimulatory molecules on DCs $(253,254)$. Specifically studies have shown that the inflammtory cytokine TNF- $\alpha$ can induce DC maturation (254). Studies analysing cytokine secretion from cDCs (255), have not assessed secretion of TNF- $\alpha$ and will therefore be presented in Chapter 4. Nonetheless, to assess the possibility of inflammatory cytokines mediating $\mathrm{pDC}$ activation, future experiments could involve sorting pDCs from Flt3L-DC cultures. The isolated pDCs could be re-cultured and stimulated with the various TLR agonists and expression of activation markers can be compared to pDCs stimulated in un-sorted cultures.

Although it is unclear whether pDC activation is influenced by the presence of cDCs, it was shown within this chapter that $\mathrm{pDC}$ depletion does not affect the maturational capacity of cDCs (Figure 3.7A). Therefore, our results indicate that cDCs does not completely rely on $\mathrm{pDC}$ interaction in order to mature following TLR stimulation.

\subsubsection{The impact of combined TLR ligation on the maturation of Flt3L- DCs}

Combining TLR agonists has been shown to enhance the maturation of GM-CSF DCs, a response in which was defined as "synergistic activation" $(235,236)$. Warger et al demonstrated that enhanced production of cytokines and co-stimulatory molecule expression were observed with GM-CSF DCs where combinations of TLR agonists induced the signalling of both the MyD88-dependent and independent pathways (236). In this chapter, we determined if combining TLR agonists that induced both MyD88dependent and independent signalling, could enhance the maturation of Flt3L-DCs.

With the exception of $\mathrm{CpG} / \mathrm{Pam} 3 \mathrm{Cys}$, all other agonist combinations used to stimulate Flt3L-DCs targeted both the MyD88-dependent and-independent signalling pathways. It was found that in comparison to stimulation with the respective individual agonists, 
combined TLR stimulation improved expression of either CD40, CD80 or both on cDCs and pDCs (Figure 3.8A, 3.8B). However, while combined TLR ligation was found to enhance expression of co-stimulatory molecules on both Flt3L-DC subtypes, we did not observe any improvement in the up-regulation of MHC II on cDCs. Given that we observed high expression levels of MHC II on cDCs following stimulation with individual agonists, this could represent the maximum level of expression that could not be further enhanced by combining TLR agonists. With respect to MHC II expression on pDCs, we did observe that the combination of $\mathrm{CpG} /$ Poly I:C, LPS/CpG, and Pam3Cys/Poly I:C to induce a slight increase in MHC II up-regulation in comparison to stimulation with the individual agonists. However, $\mathrm{CpG}$ alone already induced high expression of MHC II, thus indicating that the stimulatory effect seen in combinations containing $\mathrm{CpG}$ was mainly derived from $\mathrm{CpG}$. Therefore, it would also appear that combined TLR stimulation has a minor effect on MHC II up-regulation on pDCs.

Collectively, these results gathered imply that combined triggering of the MyD88dependent and-independent pathways could improve expression of activation markers on Flt3L-DCs, more specifically CD40 and CD86. However, it is noteworthy that the effects from combined TLR stimulation were subtle and enhanced expression by no more than two fold on cDCs and pDCs. Therefore, our results would suggest that combined TLR ligation on Flt3L-DCs induces an additive or sub-additive effect, as opposed to a synergistic response. Napolitani et al, first described the effect of combined TLR ligation as synergistic activation, after observing that GM-CSF/IL-4 DCs, simultaneously activated with a TLR3/4 and TLR7/9 agonist, induced 20 to 50 fold more production of IL-12 than did the addition of single agonists (235). Thus, to further assess whether the effect of combined TLR ligation on Flt3L-DCs could induce a synergistic response, cytokine secretion will have to be assessed. This data will be presented and further discussed in Chapter 4. 


\subsubsection{Regulation of DC activation by glucose metabolism}

Krawczyk et al demonstrated that GM-CSF DCs in the resting state derive a majority of their energy via mitochondrial $\beta$-oxidation and oxidative phosphorylation. However, stimulation with TLR agonists initiates a metabolic conversion towards aerobic glycolysis. Particularly, Krawczyk et al showed TLR mediated activation was highly dependent upon glycolytic metabolism, as treatment of LPS-stimulated GM-CSF DCs with the competitive glycolytic inhibitor 2-DG blocked the up-regulation of activation markers (245).

We observed that GMCSF/IL-4 DCs, treated with various concentrations of 2-DG alone maintained both viability and a baseline expression of activation markers comparable to unstimulated controls. When stimulated with LPS in the presence of 2-DG, viability of GMCSF/IL-4 DCs was not affected, but their ability to up-regulate activation markers was impaired (Figure 3.11A, 3.11E). Regardless of the concentration of 2-DG used to treat GMCSF/IL-4 DCs, the expression level of activation markers following LPS stimulation remained at least 2-fold lower, compared to GMCSF/IL-4 DCs stimulated with just LPS (Figure 3.11C). In fact, these expression levels were close to those displayed by the untreated GMCSF/IL-4 DCs, thus highlighting a profound block in the ability of these DCs to up-regulate activation markers. Overall, our data are consistent with the findings of Krawczyk et al. Similar to their observations in GM-CSF DCs, we observed that the maturational capacity of GMCSF/IL-4 DCs, grown in our culture conditions is also highly reliant on glycolytic metabolism.

Conversely, Flt3L-cDCs treated with a high dose of 2-DG either in the presence or absence of LPS showed decreased viability and reduced expression of activation markers (Figure 3.11B, 3.11E). At low concentrations of 2-DG, however, FLt3L-cDCs maintained viability and maturational capacity (Figure 3.11B, 3.11E). The response was similar for CD80, CD40 and MHC II levels, although CD40 levels were always lower than the LPS only stimulated control and higher than in the unstimulated Flt3L-cDCs.

Collectively, it is apparent that Flt3L-cDCs in the non-activated state display a higher sensitivity to inhibition of glucose metabolism, compared to non-activated GMCSF/IL-4 
DCs. There are two possible interpretations of this finding. Firstly, it may be indicative of the fact that energy production in Flt3L-cDCs within the resting state could be shifted more towards glycolytic metabolism. Alternatively, Flt3L-cDCs may require that all metabolic processes generating energy occur simultaneously at high levels, in order to maintain survival within the resting state. Thus, mitochondrial metabolism, which is generally the preferred route of energy metabolism during glucose limitation (256), may not be enough for resting Flt3L-cDCs. The response of Flt3L-cDCs to activation with LPS under glucose limitation also differs from GMCSF/IL-4 DCs. While the latter are unable to up-regulate activation markers at all 2-DG concentrations used, Flt3L-cDCs appear to have a threshold level somewhere between the high and intermediate concentrations of 2 DG in our experiments. If the available glucose level is below a certain threshold, FLt3LcDCs appear to be not only inhibited in activation but also in viability. In contrast, if the glucose level is above that minimum requirement, FL3L-cDCs are both viable and able to up-regulate activation markers. This indicates that while Flt3L-cDCs are still highly sensitive to glucose levels during activation they do not switch to glycolytic metabolism for activation to the same extent as GMCSF/IL-4 DCs.

In summary, glucose requirement of Flt3L-cDCs differs from GMCSF/IL-4 DCs in both the resting and activated state. Although it cannot be confirmed from the experiments described within this chapter, the data collected imply that Flt3L-cDCs are more reliant on glycolytic energy metabolism during the resting state and less reliant on glycoysis to fuel maturation. Future experiments, which may aid in determining this factor, will be further discussed in Chapter 5.

\subsection{Conculsion}

It has been shown in this chapter that our Flt3L cultures produce DC subsets that are similar to those described within the literature. Particularly, this was demonstrated in their morphological and phenotypic characteristics. Flt3L-DCs were shown to activate and induce the up-regulation of activation markers following stimulation with TLR agonists. Additionally, TLR agonist combinations that induce both the MyD88-dependent and 
independent signalling enhances their maturational response. Finally, it was demonstrated that Flt3L-DCs are less sensitive to glucose inhibition following stimulation with LPS, thus implying that energy metabolism fuelling Flt3L-DC activation may not be entirely dependent on glycolytic metabolism. 


\section{Chapter 4.}

Determining the functional capabilities of Flt3L-DCs 


\subsection{Introduction}

\subsubsection{Anti-tumour immune response requires $\mathrm{CD4}^{+}$and $\mathrm{CD8}^{+} \mathrm{T}$ cell activation}

Activation by TLR agonists initiates profound changes in DCs (257). The transition from an immature to a mature state is characterised not only by phenotypic changes, but also by changes in DC function. As discussed in Chapter 1, such changes in DC function include the ability to secrete inflammatory cytokines and to stimulate the proliferation and differentiation of $\mathrm{CD}^{+}$and $\mathrm{CD}^{+} \mathrm{T}$ cells $(66,131)$. Studies have now firmly established the importance in the initiation of both $\mathrm{CD}^{+}$and $\mathrm{CD} 4^{+} \mathrm{T}$ cell responses for effective antitumour immunity $(258,259)$. Historically, cancer-directed therapies were focused towards eliciting a $\mathrm{CD}^{+}$CTL response, due to their ability of directly killing tumour cells (260). Additionally, preferential attention were given to inducing $\mathrm{CD} 8^{+}$CTL responses, because it was found that many tumours, particularly solid tumours, are positive for MHC I molecules, but not for MHC II $(261,262)$. Therefore, given that antigen presentation on MHC II molecules is the restricting element for $\mathrm{CD}^{+} \mathrm{T}$ cell recognition, $\mathrm{CD}^{+} \mathrm{CTLs}$ were thought to serve as the dominant effector cells mediating tumour killing.

However, in recent years, it has become evident that $\mathrm{CD}^{+} \mathrm{T}$ cells also play an important role in facilitating anti-tumour immune responses. $\mathrm{CD}^{+} \mathrm{T}$ cells have been shown to be essential in the maintenance of $\mathrm{CD} 8^{+} \mathrm{T}$ cell effector functions by secreting cytokines such as IL-2, promoting $\mathrm{CD}^{+} \mathrm{T}$ cell proliferation $(262,263)$. It has also been demonstrated that $\mathrm{CD}^{+} \mathrm{T}$ cells, through the secretion of cytokines such as IL-5 and IFN- $\gamma$, induce the activation and recruitment of eosinophils and macrophages, aiding in tumour destruction by producing both superoxide and nitric oxide (264). Conversely other studies have shown that IFN- $\gamma$ induce macrophages to produce chemokines CXCL9/MIG and CXCL10/IP-10 (265, 266), which exerts anti-angiogenic activity by damaging tumour vasculature, resulting in growth inhibition and tumour necrosis $(265,267)$. Furthermore, a number of studies have shown a need for $\mathrm{CD} 4^{+} \mathrm{T}$ cells in the generation and maintenance of memory $\mathrm{CD}^{+} \mathrm{T}$ cells $(268-270)$. In conclusion, $\mathrm{CD}^{+} \mathrm{T}$ cells are a critical component of protective immunity. 


\subsubsection{The role of cytokines in anti-tumour immune responses}

Cytokines have crucial functions in the development, and regulation of immune cells, but can also play an important role in anti-tumour immunity. It has been shown in studies with GMCSF/IL-4 DCs, that stimulation with TLR agonists induces the secretion of the proinflammatory cytokines IL-12 and TNF- $\alpha$ and also the anti-inflammatory cytokine IL-10, (214). IL-12 is a major cytokine responsible for the differentiation of $\mathrm{CD}^{+} \mathrm{Th} 1$ cells, which are potent producers of the cytokine IFN- $\gamma$. Not only is IFN- $\gamma$ shown to have antitumour and anti-angiogenic activities (section 4.1.1), but it also has an enhancing effect on the ability of DCs to produce IL-12, acting therefore, as a positive feedback mechanism (271). Furthermore, IL-12 has been shown to be indispensible for $\mathrm{CD}^{+} \mathrm{T}$ cell clonal expansion and development of effector function (272). This is often called the third signal, in addition to antigen-MHC I complex recognition and (signal 1) and binding of costimulatory molecules (signal 2). TNF- $\alpha$ has also been shown to have a critical role in anti-tumour immunity, and is required for effective priming, proliferation, and recruitment of tumour-specific T cells (273). Furthermore, TNF- $\alpha$ has been shown to increase DC migration to the draining LN (274). Mature DCs can also secrete the inflammatory cytokine IL-6, which have been shown to play a critical role in $\mathrm{T}$ cell activation, by overcoming Treg-mediated suppression of T cell responses (275). Although inflammatory cytokines are important for potentiating the immune response, there must be a fine balance between immunity and detrimental systemic inflammation. Such regulation in the immune response can be derived from the cytokine IL-10, which has been shown to inhibit the production of IL-12 (276).

It is critical that Flt3L-DCs possess the functional attributes described above, if they are to be exploited for cancer immunotherapy. Studies have shown the ability of Flt3L-DCs to secrete the pro-inflammatory cytokine IL-12 $(214,233)$ and to activate and induce proliferation of both naïve $\mathrm{CD}^{+}$and $\mathrm{CD}^{+} \mathrm{T}$ cells in vitro $(225,231)$. Thus, to determine the efficacy of Flt3L-DCs generated within our system for cancer immunotherapy, it was necessary to establish their functional capabilities. Specifically, our research will not only validate the finding of other authors but will also provide new information with respect to the effects of stimulation with combined TLR agonists on Flt3L-DC function. Moreover, we also evaluate production of cytokines not reported by others, such as IL- 6 and TNF- $\alpha$. 


\subsection{Aim}

The aim of this chapter was to investigate the functional capabilities of Flt3L-DCs. To evaluate the optimum conditions of activation that may enhance Flt3L-DC function, the TLR agonist combinations that were used in Chapter 3 were further used within this chapter. As a final point of investigation, those conditions considered optimal were selected for use in tumour experiments, to observe the impact of Flt3L-DCs in immunotherapy to delay or prevent tumour growth in mice.

Specific aims within this chapter were:

1. To evaluate cytokine production by Flt3L-DCs following stimulation with TLR agonists.

2. To evaluate if cytokine production differs among the Flt3L-DC subsets.

3. To determine whether Flt3L-DCs can induce the proliferation of naïve $\mathrm{CD}^{+}$and $\mathrm{CD}^{+} \mathrm{T}$ cells in vitro.

4. To examine if Flt3L-DCs are able to cross-present to $\mathrm{CD} 8^{+} \mathrm{T}$ cells in vitro.

5. To determine if activated Flt3L-DCs loaded with tumour antigens can delay or prevent tumour growth in mice.

\subsection{Results}

\subsubsection{Activation of Flt3L-DCs with individual TLR agonists induces cytokine production}

An important functional characteristic of DCs is their ability to secrete cytokines following activation. Studies have firmly established the ability of Flt3L-DCs to produce the inflammatory cytokine IL-12 following stimulation with TLR agonists $(214,225,233)$. In addition, Flt3L-DCs have also been shown to secrete the anti-inflammatory cytokine IL-10 in some studies (233), but not in others (214). We therefore determined the ability of Flt3L-DCs generated within our culture system to secrete IL-12 and IL-10 in response to stimulation with individual TLR agonists. 
Flt3L-DCs were generated as described in Chapter 2 (2.4.2.2) and stimulated on day 9 with individual TLR agonists for 24 hours. Following stimulation, culture supernatants were collected and analysed for IL-12p70 and IL-10 using a bioplex assay (Chapter 2, (2.4.4.3)). The bioplex assay can detect the biologically active form of IL-12, which is denoted as IL12p70. IL-12 is a heterodimeric molecule composed of a p35 and p40 subunit that are linked by disulphide bonds to form the biologically active IL-12p70 (277).

Results showed that Flt3L-DCs secreted both the heterodimeric IL-12p70 and IL-10 in response to stimulation with TLR agonists (Figure 4.1). Specifically, we found that treatment with Poly I:C induced the highest amount of IL-12p70 secretion from Flt3L-DCs in comparison to those stimulated with $\mathrm{CpG}$ or Pam3Cys. However, those that were stimulated with LPS produced undetectable levels of IL-12p70, as the concentration secreted was below the range for measurement. In contrast to IL-12p70, all TLR agonists used to stimulate Flt3L-DCs induced secretion of IL-10, with $\mathrm{CpG}$ having the most pronounced affect (Figure 4.1). In summary, we found that Flt3L-DCs secreted both proinflammatory and anti-inflammatory cytokines following TLR stimulation. 

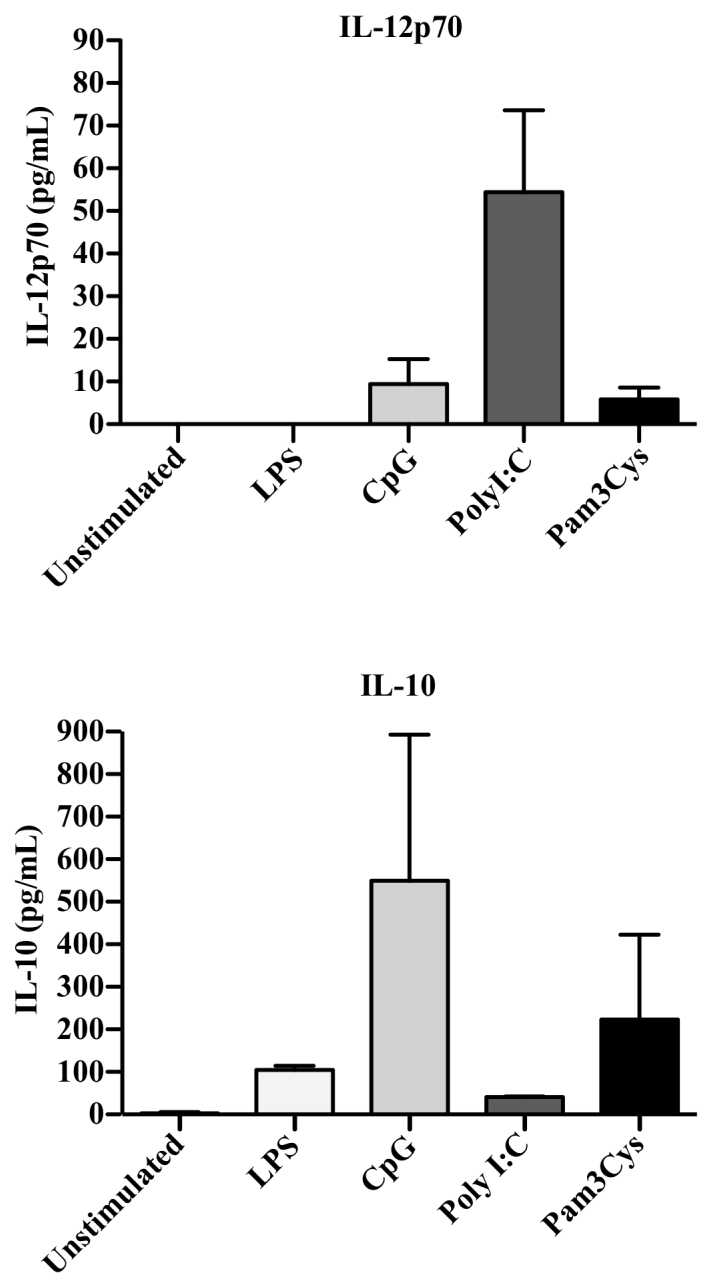

Figure 4.1: Flt3L-DCs activated with TLR agonists produce pro-inflammatory and anti-inflammatory cytokines. BM cells derived from C57BL/6 mice were cultured in the presence of Flt3L for 9 days to induce DC differentiation. Flt3L-DCs were stimulated on day 9 with TLR agonists at the following concentrations: LPS (100ng/mL), CpG $(2 \mu \mathrm{g} / \mathrm{mL})$, Poly I:C $(50 \mu \mathrm{g} / \mathrm{mL})$ and Pam3Cys $(1 \mu \mathrm{g} / \mathrm{mL})$ and incubated for 24 hours. Following stimulation, DC culture supernatants were collected and analysed for IL-12p70 or IL-10 using a bioplex assay. Bar graph values represent mean \pm S.D. $*=0.01<P<0.05, * * *=$ $\mathrm{P}<0.001$ as determined by the Kruskal-Wallis with Dunn's multiple comparison's test. Results show averages of duplicate samples from two independent experiments.

\subsubsection{Selected TLR agonist combinations enhance the secretion of pro- nflammatory cytokines}

To further assess the influence of combined TLR ligation on Flt3L-DCs, we sought to determine whether activation with combined TLR agonists could enhance secretion of pro- 
inflammatory cytokines IL-12p70, TNF- $\alpha$ and IL-6. Analysis for IL-10 production was not performed, as detection kits for this cytokine were not available at the time of experiment.

Flt3L-DCs were generated as described in Chapter 2 (2.4.2.2) and stimulated on day 9 with the individual or combined TLR agonists for 24 hours. Following stimulation, DC culture supernatants were collected and analysed for the cytokines indicated above using a bioplex assay (Chapter 2, (2.4.4.3)).

The results obtained from the bioplex assay showed that in addition to secreting IL-12p70, Flt3L-DCs secreted both TNF- $\alpha$ and IL-6 in response to stimulation with individual TLR agonists (Figure 4.2). Unlike in the cytokine data in Figure 4.1, Flt3L-DCs stimulated with LPS secreted detectable (although variable) levels of IL-12p70. We were unsure as to the cause of variation in between the two experiments. In contrast to stimulation by individual agonists, some TLR agonist combinations did enhance secretion of IL-12p70 (Figure 4.2). Specifically, these were combinations of Poly I:C with either Pam3Cys or CpG. A slight increase in the production of IL-12p70 was also observed with Flt3L-DCs stimulated with LPS/Poly I:C. Only the combination of Pam3Cys/Poly I:C and LPS/CpG were observed to induce more secretion of TNF- $\alpha$ and IL-6, respectively, however the response to these agonist combinations were heterogeneous.

On the basis of this experiment and those that previously evaluated the effect of combined TLR ligation on the up-regulation of activation markers (Chapter 3), we selected an optimal agonist combination for inducing Flt3L-DC activation in subsequent experiments. Of all the combinations tested, Pam3Cys/Poly I:C or CpG/Poly I:C were likely candidates, given their ability to enhance production of IL-12p70. However, because our cytokine data indicated that stimulation with Pam3Cys/Poly I:C could enhance production of TNF$\alpha$ (Figure 4.2), we proceeded to select this combination over $\mathrm{CpG} /$ Poly I:C. Furthermore, stimulation with Pam3Cys/Poly I:C also enhanced the up-regulation of activation markers on Flt3L-DCs (Chapter 3, Figure 3.7), and was therefore optimal. 

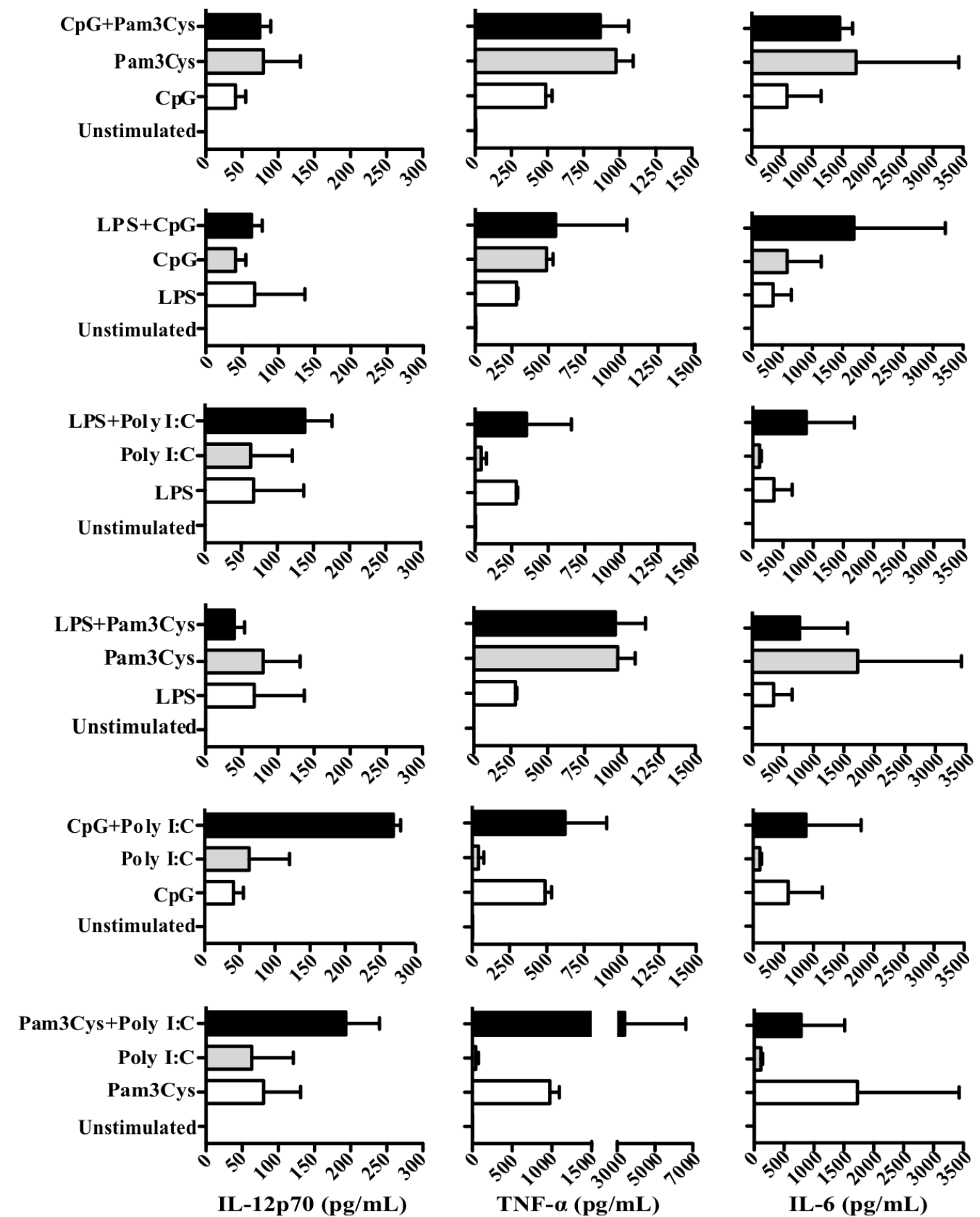

Figure 4.2: Selected TLR agonist combinations enhance the secretion of proinflammatory cytokines from Flt3L-DCs. Flt3L-DCs were generated as described in Figure 4.1 and stimulated on day 9 with TLR agonists at the following concentrations: LPS (100ng/mL), CpG $(2 \mu \mathrm{g} / \mathrm{mL})$, Poly I:C $(50 \mu \mathrm{g} / \mathrm{mL})$ and Pam3Cys $(1 \mu \mathrm{g} / \mathrm{mL})$. Following 24 hours of stimulation, DC culture supernatants were collected for analysis of the indicated cytokines by a bioplex assay. Bar graph values represent mean \pm S.D. Results are pooled data from 3 independent experiments. Statistical significance was tested using the Kruskal-Wallis with Dunn's multiple comparisons test. No significance was detected. 


\subsubsection{Flt3L-DC subsets differ in their capacity to secrete pro- inflammatory cytokines}

As shown in Figure 4.2, we observed that Flt3L-DCs secreted pro-inflammatory cytokines following stimulation with TLR agonists. However, it was of interest to study whether the Flt3L-DC subsets, generated from our cultures differed in their capacity to produce proinflammatory cytokines, and at which time point. Thus, using a method of intracellular staining, production of IL-12p40 was evaluated in Flt3L-DCs following 6 hour and 24 hour stimulation with TLR agonists. Intracellular staining measures the expression of cytokines within the cells and was used in combination with cell surface marker staining to allow for detection of cytokine production in different DC subpopulations by flow cytometry. There are currently no antibodies available to detect murine IL-12p70, thus intracellular detection of IL-12 was performed on the p40 subunit (IL-12p40).

Flt3L-DCs were generated as described in Chapter 2 (2.4.2.2) and stimulated on day 9 with the agonist combination of Pam3Cys/Poly I:C or the respective individual TLR agonists for 6 or 24 hours. For the last 4 hours of stimulation, cytokine secretion was inhibited by treatment with GolgiStop. Following stimulation, cells were harvested and labelled with antibodies against cell surface makers. Subsequently, cells were permeablised and stained with antibodies against the cytokines or the respective isotype control antibodies (as described in 2.4.5.1 and 2.4.5.2, respectively). Cells were then analysed by flow cytometry. The $\mathrm{cDC}$ and $\mathrm{pDC}$ subsets were identified by negative and positive expression of the marker B220, respectively. The cDC population was then further separated into two subsets that were CD11 $b^{\text {high }}$ and CD24 $4^{\text {high }}$ (Figure 4.3A).

The CD $24^{\text {high }}$ and CD $11 b^{\text {high }}$ cDCs were the primary subsets producing IL-12p40 following stimulation with the selected individual or combined TLR agonists (Figure 4.3B). More specifically, we observe that production of IL-12p40 from the CD11b ${ }^{\text {high }}$ cDCs stimulated with Poly I:C, was only detected 24 hours after stimulation. Conversely, the CD24 $4^{\text {high }}$ cDCs stimulated with Poly I:C, exhibited decreased production of IL-12p40 at 24 hours. Overall, the production of IL-12p40 from the cDC subsets was higher at 6 hours, but production continued until the 24 hour time point. No production of IL-12p40 was observed from $\mathrm{pDCs}$. 


\section{Figure 4.3: Production of IL-12p40 is detected in cDCs, but not in pDCs.}

Flt3L-DCs were generated as described in Figure 4.1. Stimulation with Pam3Cys/Poly I:C or the respective individual agonists occurred on day 9 for 24 hours, or on day 10 for 6 hours at the following concentrations: Poly I:C $(50 \mu \mathrm{g} / \mathrm{mL})$ and Pam3Cys $(1 \mu \mathrm{g} / \mathrm{mL})$. For the final 4 hours of stimulation, Flt3L-DCs were treated with GolgiStop to inhibit secretion of IL-12p40. Following stimulation, cells were harvested, stained with the viability dye Live Dead Fixable Blue (LDFB) and labelled with antibodies against the cell surface markers CD11c, B220, CD11b, CD24. Cells were subsequently stained with antibodies against the cytokines IL-12p40, or with the respective isotype control antibody, and analysed by flow cytometry. (A) General gating strategy of Flt3L-DC subsets is shown. Cells of interest were selected based on analysis of SSC and FSC properties. This was then followed by gating of live cells, which were determined by those excluding the viability dye LDFB. The CD11 $\mathrm{c}^{+}$population was gated, and pDCs were identified by expression of B220. The $\mathrm{CDC}$ population was gated based on negative expression of B220 and was further subdivided into two subsets that were $\mathrm{CD} 11 \mathrm{~b}^{\text {high }}$ and $\mathrm{CD} 24^{\text {high }}$. Numbers indicate percentages of cells within the respective gating regions. (B) Detection of IL-12p40 production in Flt3L-DC subsets. Results are from one of two independent experiments with similar results. 
A) Gating strategy
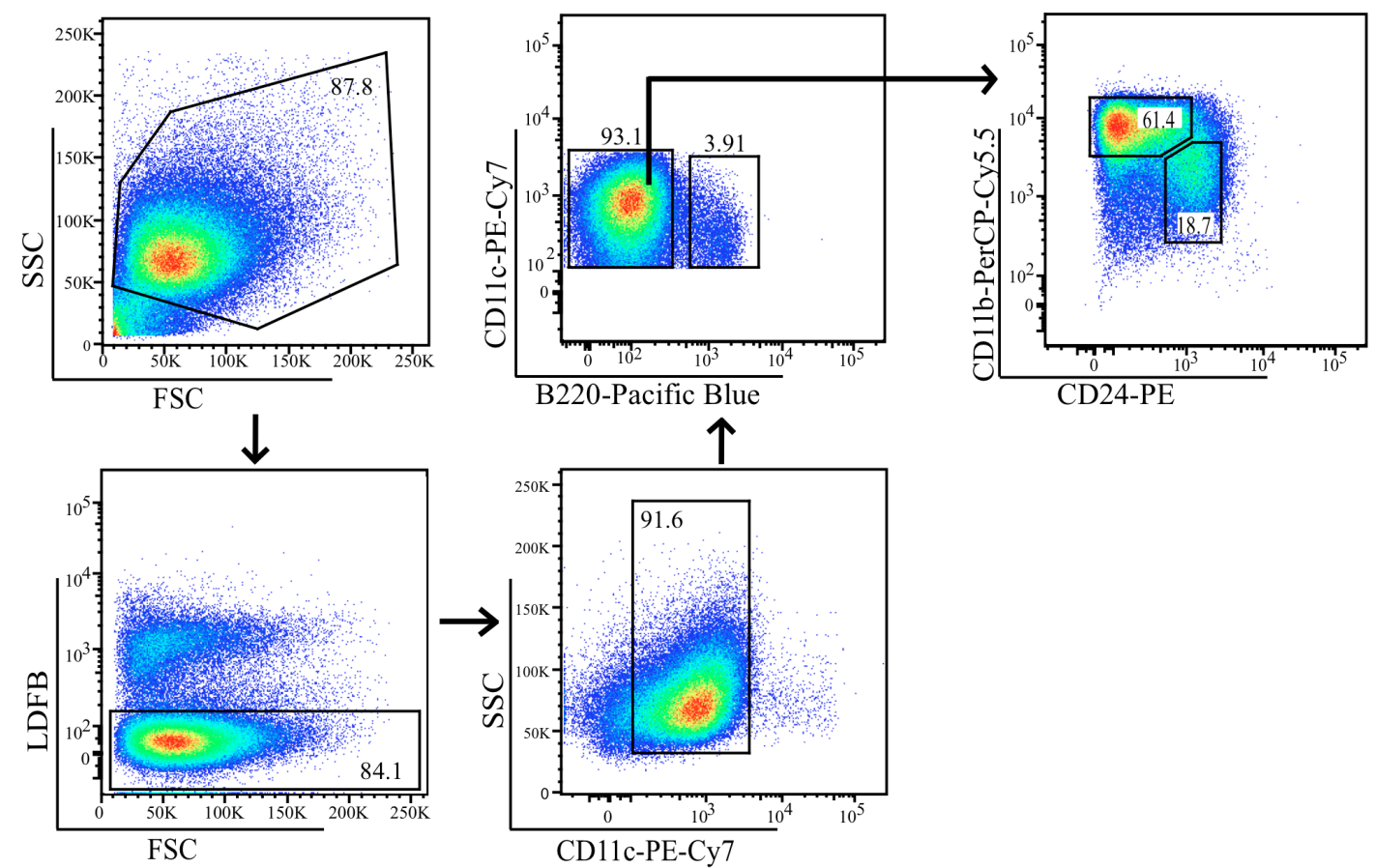
B) $\mathrm{IL}-12 \mathrm{p} 40$
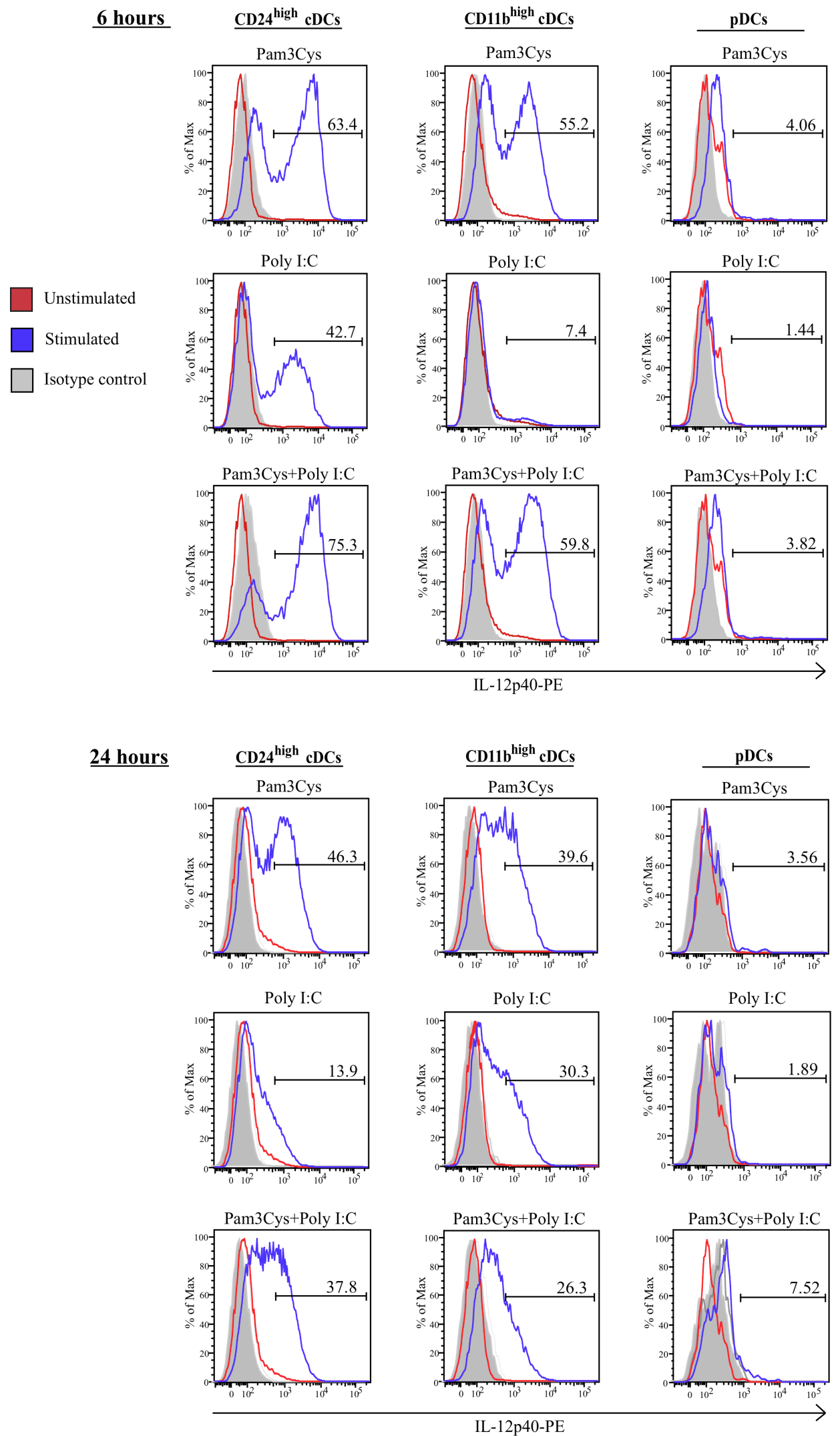
The kinetics of TNF- $\alpha$ production from Flt3L-DCs generated in vitro has not been reported. Therefore we also investigated whether TNF- $\alpha$ production differed among the Flt3L-DC subsets. Flt3L-DCs were generated as described in Chapter 2 (2.4.2.2) and stimulated on day 9 with the combined TLR agonists or the individual agonists for 6 or 24 hours. Subsequent experimental procedures used for detecting TNF- $\alpha$ among the DC subsets was performed as described for IL-12p40.

Production of TNF- $\alpha$ was only detected within the CD24 $4^{\text {high }}$ cDCs stimulated with Pam3Cys and Poly I:C, at 6 hours. Interestingly, TNF- $\alpha$ could not be detected within the same subset stimulated with the respective individual TLR agonists. (Figure 4.4A). This observation does not support data shown in Figure 4.2, whereby TNF- $\alpha$ secretion was detected in Flt3L cultures stimulated with the individual TLR agonists. Furthermore, the CD $11 b^{\text {high }}$ cDCs and pDCs were not observed to produce TNF- $\alpha$ overall. Due to this result, we asked whether TNF- $\alpha$ could not be detected due to insufficient inhibition of TNF- $\alpha$ secretion. GolgiStop inhibits cytokine secretion by targeting the trans-Golgi function. However, Brefeldin A can also be used for inhibiting cytokine secretion, and function by inhibiting protein transport between the ER and the Golgi (278). Thus, we subsequently determined if the addition of both GolgiStop and Brefeldin A would influence the outcome of intracellular detection of TNF- $\alpha$ within Flt3L-DCs.

Combining GolgiStop and Brefeldin A resulted in detection of TNF- $\alpha$ from the CD24 $4^{\text {high }}$ cDCs stimulated with the individual and combined TLR agonists at 6 hours, but not 24 hours (Figure 4.4B). Stimulation with the combined TLR agonists also enhanced TNF- $\alpha$ production, as evidenced by an increase in the percentage of TNF- $\alpha^{+}$CD $24^{\text {high }}$ cDCs. TNF- $\alpha$ production was not detected within $\mathrm{CD} 11 \mathrm{~b}^{\text {high }} \mathrm{cDCs}$ and $\mathrm{pDCs}$ under any conditions tested. Therefore, TNF- $\alpha$ is only produced by the CD2 $4^{\text {high }} \mathrm{cDCs}$, at an early time point after stimulation.

Overall, these results demonstrated the differential capacity of Flt3L-DCs to secrete IL12 p40 and TNF- $\alpha$ following stimulation with the selected TLR agonists. Based on this observation, the following experiments presented within this chapter involve depletion of 
$\mathrm{B} 220^{+}$pDCs from Flt3L cultures, in order to narrow the focus to cDCs, which are the optimal subsets producing pro-inflammatory cytokines. The cDC subset are thus referred to as Flt3L-cDCs from here on. 
Figure 4.4: TNF- $\alpha$ production is only detected in CD24 ${ }^{\text {high }}$ cDCs. Flt3L-DCs were generated as described in Figure 4.1. Stimulation with Pam3Cys/Poly I:C or the respective individual agonists occurred on day 9 for 24 hours, or on day 10 for 6 hours at the following concentrations: Poly I:C $(50 \mu \mathrm{g} / \mathrm{mL})$ and Pam3Cys $(1 \mu \mathrm{g} / \mathrm{mL})$. For the final 4 hours of stimulation, Flt3L-DCs were treated with (A) GolgiStop or (B) GolgiStop and Brefeldin A to inhibit secretion of TNF- $\alpha$. Following stimulation, cells were harvested and labelled with antibodies against cell surface markers CD11c, B220, CD11b and CD24. Cells were subsequently stained with antibodies against the cytokines TNF- $\alpha$ or with the respective isotype control antibody and analysed by flow cytometry. (A) and (B) represents one out of two independent experiments with similar results. 
A) TNF- $\alpha$ - GolgiStop only

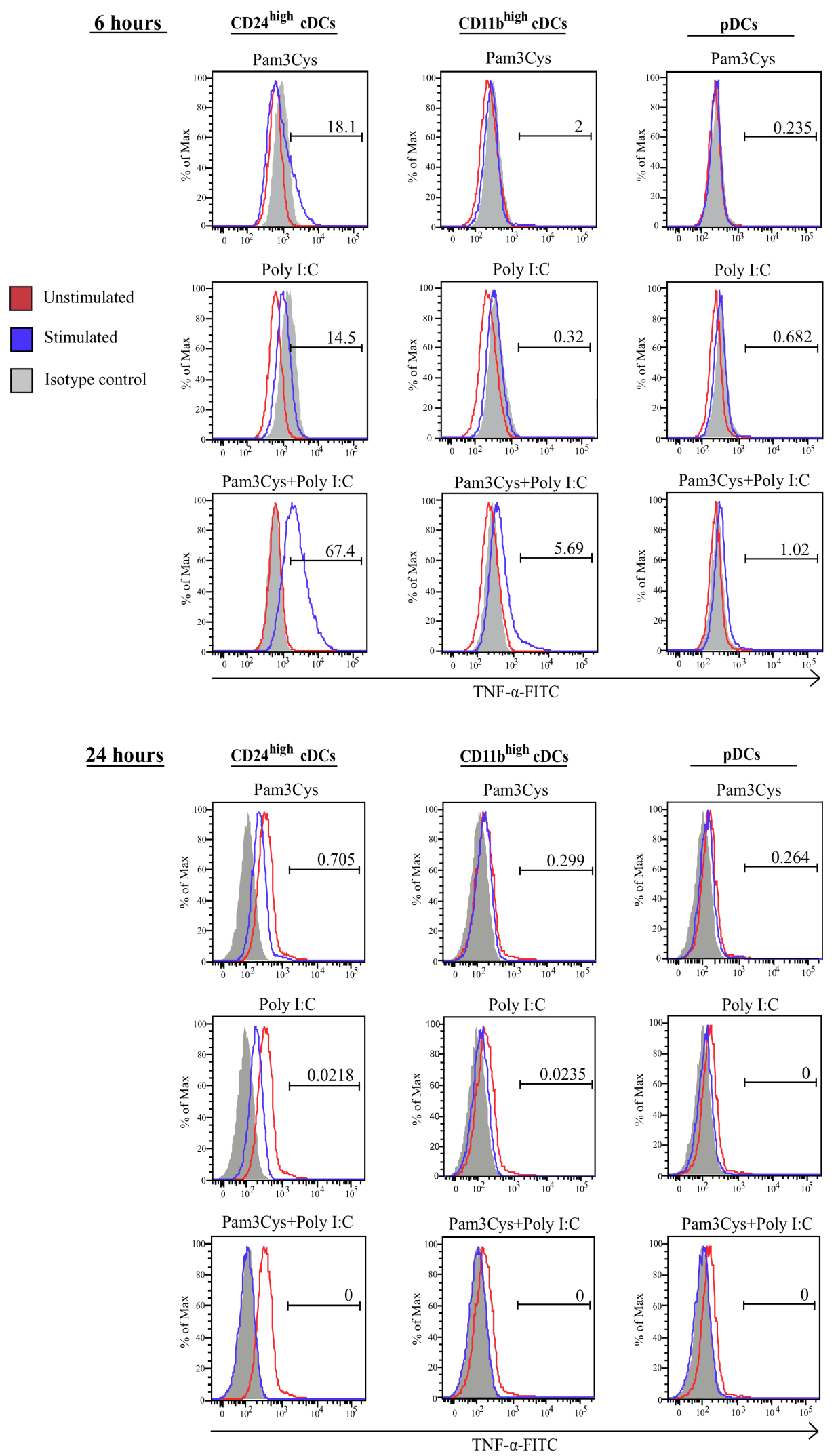


B) TNF- $\alpha$ - GolgiStop + Brefeldin A

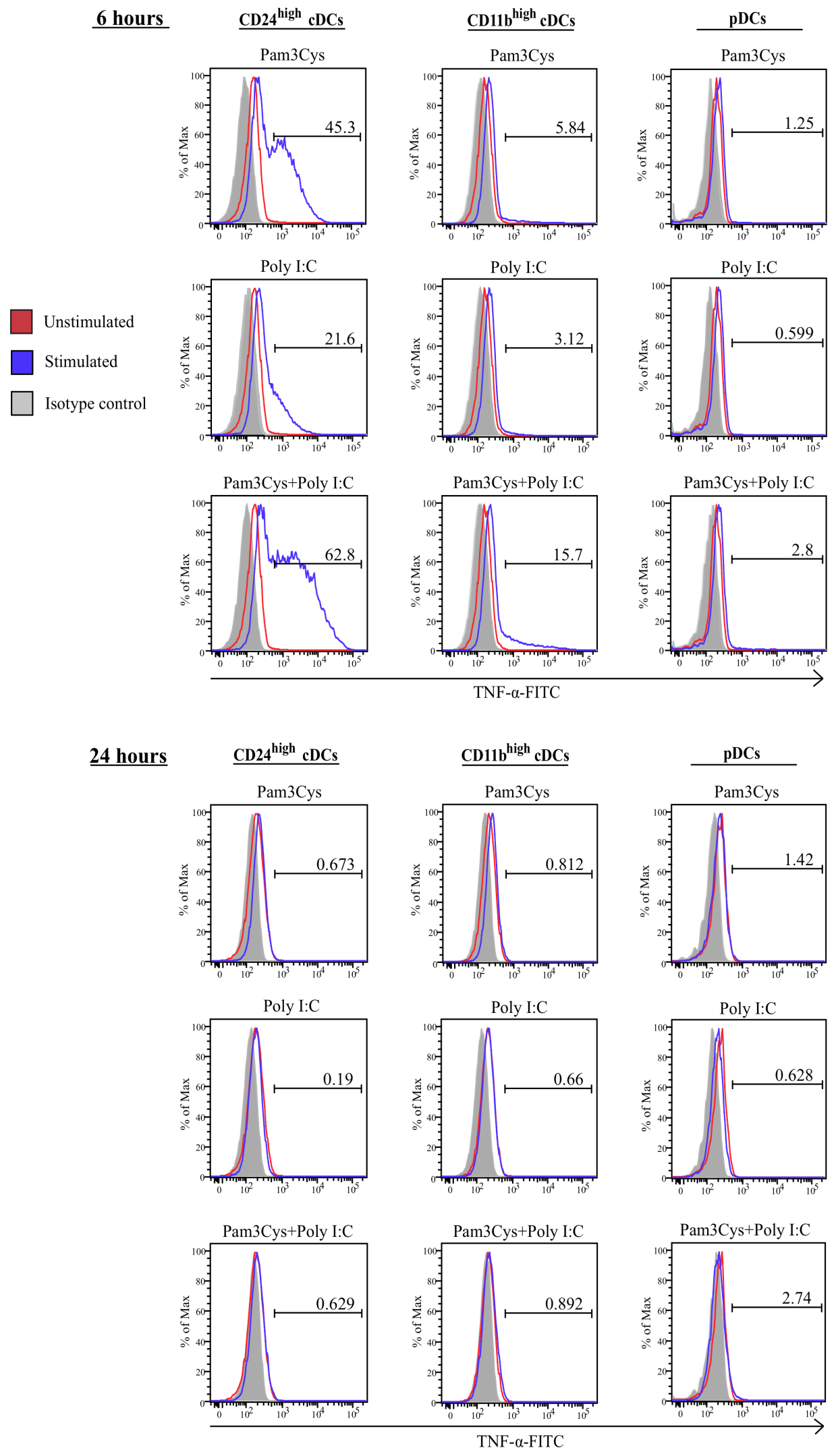




\subsubsection{Stimulation with combined TLR agonists improves the ability of FIt3L-cDCs to induce $\mathrm{CD}^{+} \mathrm{T}$ cell proliferation}

To initiate immune responses, DCs need to activate naïve $\mathrm{T}$ cells and induce their proliferation $(131,279)$. For effective $\mathrm{T}$ cell activation, DCs must be capable of antigen presentation, in parallel of having undergone maturation. In this chapter, we sought to determine the ability of Flt3L-DCs stimulated with the combined or respective single TLR agonists to present peptide antigen and induce the activation and proliferation of naïve $\mathrm{CD} 4^{+}$and $\mathrm{CD} 8^{+} \mathrm{T}$ cells in vitro.

Flt3L-DCs were generated, as described in Chapter 2 (2.4.2.2), and depleted of B220 pDCs on day 9 (Chapter 2, (2.4.3.3)). Following depletion, Flt3L-cDCs were placed back into culture and stimulated with combined or individual TLR agonists. DCs were harvested after 24 hours, loaded with either SIINFEKL $(0.3 \mathrm{nM})$ or ISQ $(0.5 \mathrm{mM})$ peptide and serially titrated onto 96-well culture plates. The concentrations used for SIINFEKL or ISQ were selected based on previous peptide titration assays (Evelyn Hyde, Malaghan Institute of Medical Research, unpublished data), which indicated that these concentrations were sufficient to induce a clear $\mathrm{T}$ cell response, but not maximal proliferation which can limit the observation of potential differences between the TLR agonist treated groups. Purified naïve OTI $\mathrm{CD}^{+}$or OT-II $\mathrm{CD}^{+} \mathrm{T}$ cells recognising SIINFEKL or ISQ, respectively, were obtained by magnetic cell separation, as described in Chapter 2 (2.4.3.2). The $\mathrm{T}$ cells were added to the DC suspensions and incubated for 48 hours. A selected proportion of the DC suspensions did not receive T cells as a DC only control. Following incubation, $\left[{ }^{3} \mathrm{H}\right]$-thymidine was added for a further 18 hours and $\mathrm{T}$ cell proliferation was evaluated by measure of thymidine uptake.

It was observed that Flt3L-cDCs stimulated with the individual TLR agonists induced a greater level of $\mathrm{CD}^{+}$and $\mathrm{CD} 8^{+} \mathrm{T}$ cell proliferation in comparison to unstimulated Flt3LcDC (Figure 4.5). With respect to $\mathrm{CD}^{+} \mathrm{T}$ cell proliferation, Flt3L-cDCs stimulated with Poly I:C slightly out-performed those stimulated with Pam3Cys. However, in comparison to Flt3L-cDCs treated with single TLR agonists, Flt3L-cDCs stimulated with the combined TLR agonists induced a stronger proliferation of $\mathrm{CD}^{+} \mathrm{T}$ cells. In contrast, Flt3L-cDCs stimulated with either Pam3Cys or Poly $\mathrm{I}: \mathrm{C}$ induced similar levels of $\mathrm{CD}^{+} \mathrm{T}$ cell 
proliferation. Moreover, we observed no difference in the level of $\mathrm{CD} 8^{+} \mathrm{T}$ cell proliferation induced by Flt3L-cDCs stimulated with the combined or individual TLR agonists.

In summary, we found that Flt3L-cDCs could present peptide antigen and induce the proliferation of $\mathrm{CD}^{+}$or $\mathrm{CD} 8^{+} \mathrm{T}$ cells in vitro. Furthermore, Flt3L-cDCs stimulated with the combined TLR agonists induced enhanced proliferation of $\mathrm{CD}^{+} \mathrm{T}$ cells, but not of $\mathrm{CD}^{+} \mathrm{T}$ cells. 

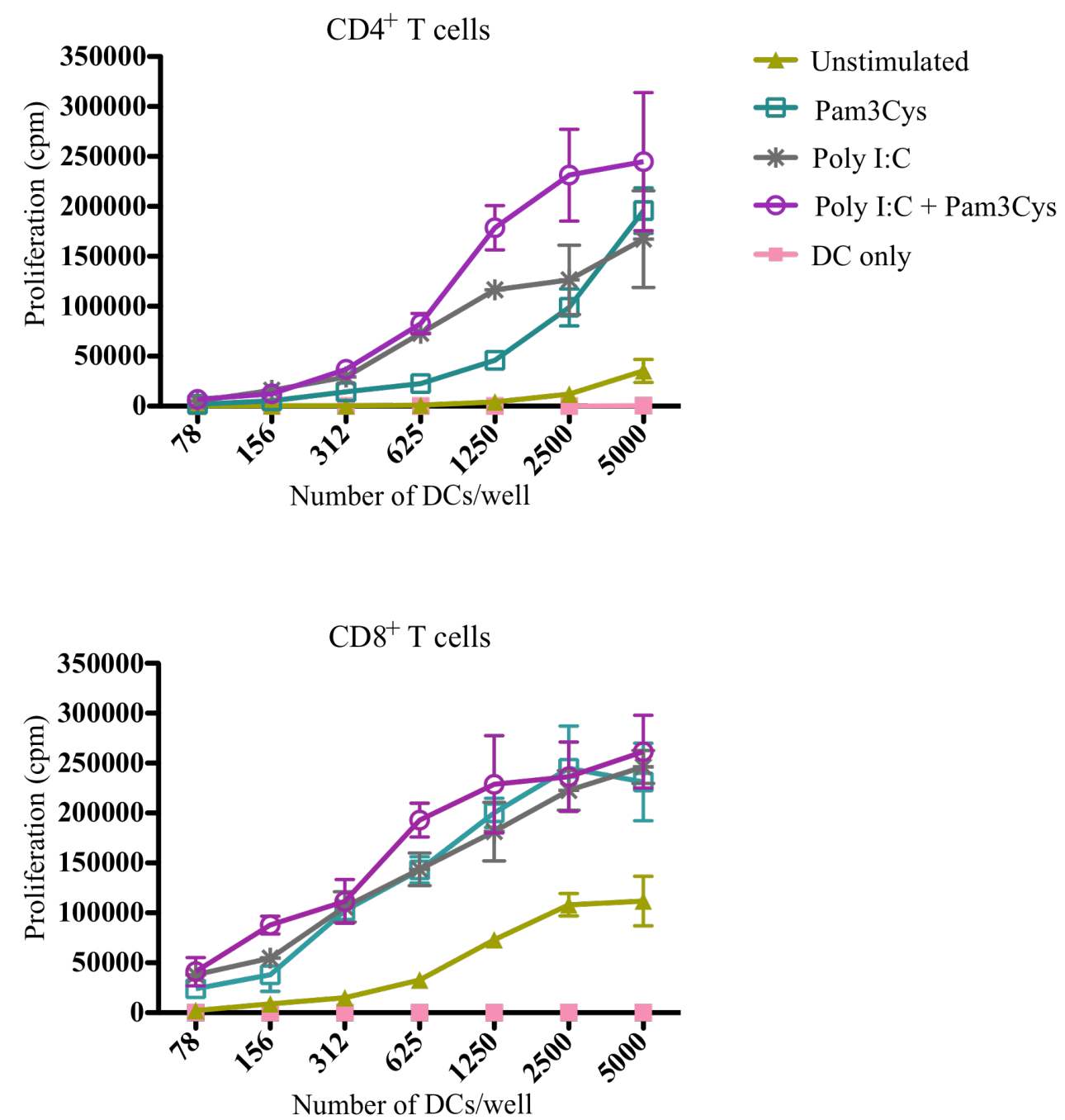

Figure 4.5: Flt3L-cDCs stimulated with combined TLR agonists acquire greater capabilities to induce proliferation of $\mathrm{CD4}^{+}$but not $\mathrm{CD8}^{+} \mathrm{T}$ cells. Flt3L-DCs were generated as described in Figure 4.1. DCs were then harvested on day 9 of cell culture and depleted of $\mathrm{B} 220^{+}$pDCs (Chapter 2, (2.4.3.3)). Flt3L-cDCs were placed back into culture and stimulated with the combined or individual TLR agonists at the following concentrations: Poly I:C $(50 \mu \mathrm{g} / \mathrm{mL})$ and Pam3Cys $(1 \mu \mathrm{g} / \mathrm{mL})$. Following 24 hours of stimulation, DCs were harvested, loaded with SIINFEKL (0.3nM) or ISQ $(0.5 \mathrm{mM})$ peptide and serially titrated onto 96 -well culture plates. Naïve OT-I CD8 ${ }^{+}$or OT-II CD4 ${ }^{+} \mathrm{T}$ cells were added to the DC suspensions at $1 \times 10^{5} \mathrm{~T}$ cells per well and were incubated for 48-72 hours. Some DC wells did not receive $\mathrm{T}$ cells as a control (DC only). Following incubation, $1 \mu \mathrm{Ci} /$ well $\left[{ }^{3} \mathrm{H}\right]$-thymidine was added to each well and incubated for a further 18 hours. Thymidine uptake in OT-I CD $8^{+}$or OT-II $\mathrm{CD}^{+}{ }^{+} \mathrm{T}$ cells was then measured. Results represent one out of two independent experiments with similar results. Each group is average data from triplicate samples. Graph values represent mean \pm S.D. 


\subsubsection{FIt3L-cDCs stimulated with combined TLR agonists are better at cross-presentation}

With the knowledge that Flt3L-cDCs could directly present peptide antigen to T cells and induce their proliferation (Figure 4.5), we subsequently sought to determine whether Flt3L-cDCs were capable of cross-presentation. Unlike the OVA peptide antigens used for the $\mathrm{T}$ cell proliferation assay, the antigen used to assess cross-presentation was soluble OVA protein. Thus, Flt3L-cDCs were evaluated for their ability to take up, process and cross-present exogenous soluble OVA to $\mathrm{CD}^{+} \mathrm{T}$ cells.

The cross-presentation assay was performed as illustrated in Figure 4.6A. Briefly, Flt3LDCs were generated from BM cells of TLR4-/- mice, as described in Chapter 2 (2.4.2.2). BM cells from TLR4-/- mice were specifically used within this assay to exclude the effects of endotoxin (LPS) within the OVA protein, which can induce the activation of Flt3LcDCs (as established in Chapter 3, (3.3.3)). This allowed us to directly assess the effects of combined or individual TLR stimulation on the cross-presentation capacity of Flt3LcDCs. On day 8 of cell culture, OVA was added at $0.5 \mathrm{mg} / \mathrm{mL}$ and incubated overnight (14-15 hours). OVA protein was added to Flt3L-DC cultures prior to stimulation with TLR agonists because DCs down-regulate internalization of antigen following maturation (280). DCs were harvested following incubation with OVA, depleted of B220 ${ }^{+}$pDCs (Chapter 2, (2.4.3.3)) and Flt3L-cDCs were placed back into culture and stimulated with TLR agonists for 24 hours. Following stimulation, Flt3L-cDCs were harvested and a selected proportion was loaded with $0.3 \mathrm{nM}$ of SIINFEKL peptide and serially titrated onto 96-culture plates. OT-I CD ${ }^{+} \mathrm{T}$ cells were added and incubated for 48 hours. $\left[{ }^{3} \mathrm{H}\right]-$ thymidine was added for a further 18 hours and $\mathrm{T}$ cell proliferation was evaluated by measure of thymidine uptake.

Results obtained from the assay showed that Flt3L-cDCs were capable of cross-presenting soluble OVA protein to $\mathrm{CD}^{+} \mathrm{T}$ cells (Figure 4.6B). We observed that unstimulated Flt3LcDCs displayed the capacity of cross-presentation, however with lower efficiency compared to Flt3L-cDCs stimulated with the individual TLR agonists. Furthermore, Flt3L-cDCs stimulated with the combined TLR agonists induced the highest level of CD8 ${ }^{+}$ $\mathrm{T}$ cell proliferation overall. Taken together, this result indicated that Flt3L-cDCs 
stimulated with combined TLR agonists have enhanced capability of cross-presentation when compared to Flt3L-cDCs stimulated with the individual TLR agonists.

The difference in $\mathrm{CD}^{+} \mathrm{T}$ cell proliferation between the combined or individual TLR agonist treated DCs could be due to differences in the cross-presentation of soluble OVA, or to the level of co-stimulation delivered to the $\mathrm{CD}^{+} \mathrm{T}$ cells. As established in Chapter 2, Flt3L-cDCs stimulated with Pam3Cys/Poly I:C showed increased levels of co-stimulatory molecule expression, which may have contributed to the increase in proliferation observed (Figure 4.6B). Therefore, to determine whether the difference in proliferation was simply due to the differential level of co-stimulation, Flt3L-DCs were generated and loaded with OVA in the same manner as described above, however, a proportion of DCs (that were harvested following 24 hour stimulation with the TLR agonists) were loaded with SIINFEKL peptide. The peptide is loaded directly onto MHC I and presented to $\mathrm{CD} 8^{+} \mathrm{T}$ cells, therefore bypassing antigen uptake and processing.

It was observed that Flt3L-cDCs stimulated with the combined or individual TLR agonists and loaded with SIINFEKL peptide induced similar levels of $\mathrm{CD} 8^{+} \mathrm{T}$ cell proliferation (Figure 4.6C). Thus, this finding indicated that the difference in cross-presentation of Flt3L-cDCs stimulated with either the combined or individual TLR agonists were most likely due to differences in antigen uptake and processing. 
A)

\begin{tabular}{|c|c|c|c|c|c|}
\hline Days 0 & $8 \rightarrow{ }_{\text {incl }}^{14}$ & 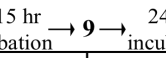 & $\underset{\text { ation }}{\mathrm{hr}} \rightarrow \mathbf{1 0} \rightarrow{ }_{\text {incul }}^{48}$ & ation $\rightarrow 12 \rightarrow{ }_{\text {inc }}^{1}$ & $8 \mathrm{hr} \rightarrow 14$ \\
\hline $\begin{array}{c}\text { Set up } \\
\text { Flt3L-DC } \\
\text { cultures from } \\
\text { TLR4 -/-mice }\end{array}$ & $\begin{array}{c}0.5 \mathrm{mg} / \mathrm{mL} \\
\text { OVA portein } \\
\text { added to DC } \\
\text { culture }\end{array}$ & $\begin{array}{l}\text { Depleted of } \\
\text { pDCs } \\
\text { Re-plated and } \\
\text { stimulated with } \\
\text { TLR agonists }\end{array}$ & $\begin{array}{c}\text { DCs harvested } \\
\text { (a portion pulsed } \\
\text { with SIINFEKL) } \\
\text { and serially } \\
\text { titrated onto } \\
\text { 96-well plates } \\
+ \\
\text { Addition of } \\
\text { CD8 }{ }^{+} \mathrm{T} \text { cells }\end{array}$ & $\begin{array}{c}\text { Addtion of } \\
{\left[{ }^{3} \mathrm{H}\right] \text {-thymidine }}\end{array}$ & $\begin{array}{l}\text { Cells harvested } \\
\text { and analysed }\end{array}$ \\
\hline
\end{tabular}
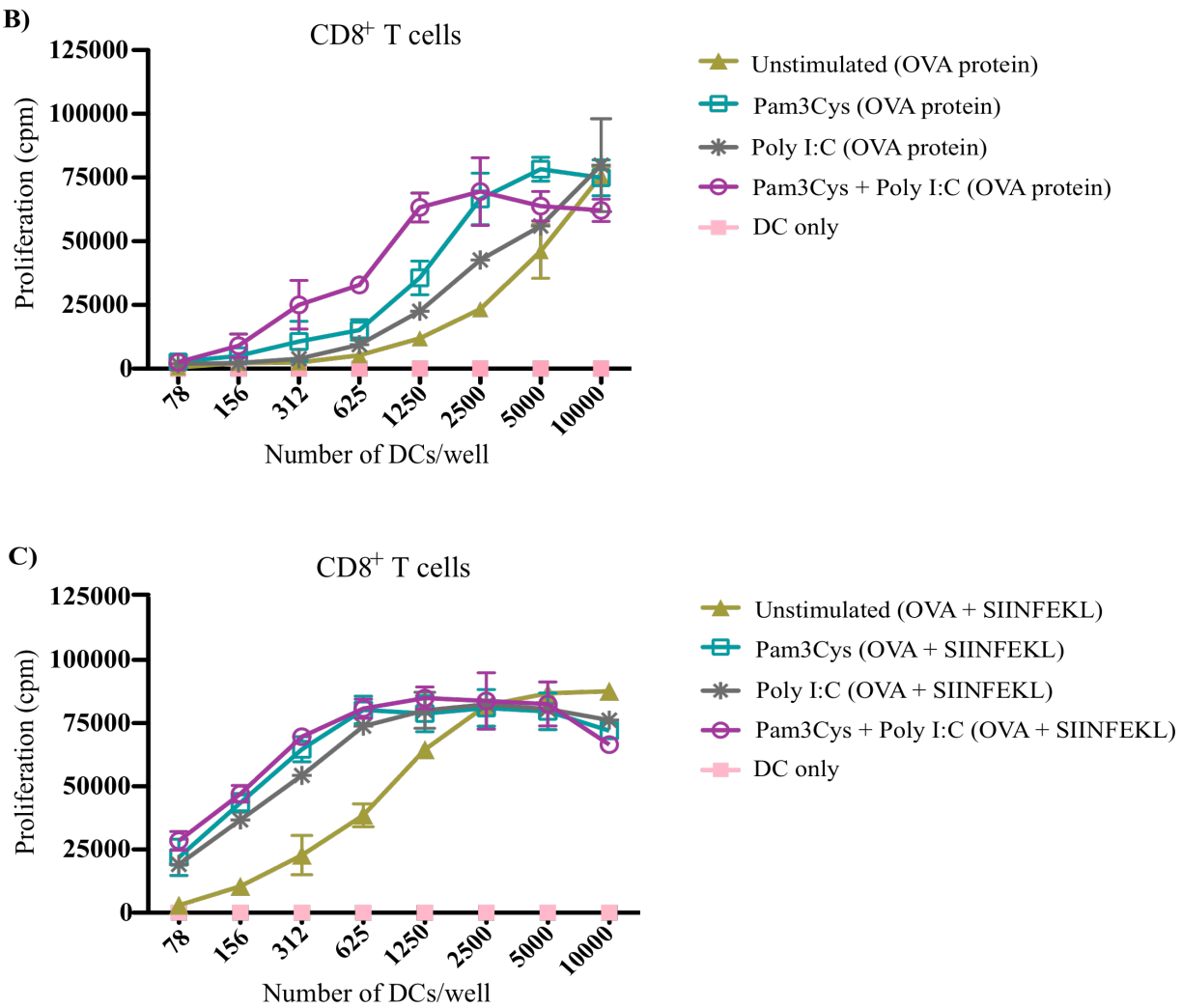

Figure 4.6: Combined TLR ligation enhances the capability of Fit3L-cDCs to cross-

present OVA. BM cells extracted from TLR4-/- mice were cultured in the presence of Flt3L to generate Flt3L-DCs. On day $8,0.5 \mathrm{mg} / \mathrm{mL}$ of OVA protein was added to the Flt3L cultures and incubated overnight (14-15 hours). Flt3L-DCs were then harvested and depleted of B220 ${ }^{+}$pDCs (Chapter 2, (2.4.3.3)). Flt3LcDCs were placed back into culture and stimulated with the combined or individual TLR agonists at the following concentrations: Poly I:C $(50 \mu \mathrm{g} / \mathrm{mL})$ and Pam3Cys $(1 \mu \mathrm{g} / \mathrm{mL})$. Following 24 hours of stimulation, Flt3L-cDCs were harvested and a proportion was loaded with $0.3 \mathrm{nM}$ of SIINFEKL peptide. DCs were serially titrated onto 96-well culture plates and $1 \times 10^{5}$ naïve OTI $\mathrm{CD}^{+} \mathrm{T}$ cells were added to the DC suspension. Cells were incubated for 48 hours, and $1 \mu \mathrm{Ci} /$ well of $\left[{ }^{3} \mathrm{H}\right]$-thymidine was then added for a further 18 hours. T cell proliferation was evaluated by measure of thymidine uptake. (A) Timeline depicts experimental procedure of the cross-presentation assay. (B) Proliferation of $\mathrm{CD}^{+} \mathrm{T}$ cells induced by Flt3LcDCs cross-presenting OVA. (C) Proliferation of $\mathrm{CD}^{+} \mathrm{T}$ cells induced by Flt3L-cDCs loaded with OVA protein and SIINFEKL peptide. Average proliferation of triplicate samples from one experiment is shown. Graph values represent mean \pm S.D. 
Herein, the results presented have demonstrated the functional capability of Flt3L-cDCs to secrete pro-inflammatory cytokines and induce $\mathrm{T}$ cell proliferation in vitro. More importantly, it has been shown that stimulation with Pam3Cys/Poly I:C not only enhances the activation status of Flt3L-cDCs, as judged by activation markers (Chapter 2), but also in their function. Therefore, an optimised condition for inducing Flt3L-DC activation has been identified. The further experiments outlined within this chapter will address the final aim of this thesis, can Flt3L-cDCs, activated with the optimised conditions, protect mice from tumour growth?

\subsubsection{Vaccination with $1 \times 10^{5}$ Flt3L-cDCs can induce tumour protection in mice}

To assess the efficacy of Flt3L-cDCs stimulated with the selected TLR agonist combination as immunotherapy against cancer, we first determined the number of DCs, required to induce protection in mice. Particularly, GMCSF/IL-4 DCs were used as a mean to compare the efficacy of vaccination with Flt3L-cDCs. Therefore, to achieve comparable activation status between the two DC populations, GMCSF/IL-4 DCs were stimulated with the agonist combination of LPS/Pam3Cys. This combination was selected based on personal communications with Dianne Sika-Paotonu (Vaccine Research Group, Malaghan Institute of Medical Research), who has established the optimal combination to induce activation of GMCSF/IL-4 DCs.

Experimental procedures were performed as outlined in Figure 4.7A. Briefly, BM cells extracted from C57BL/6 mice were cultured in Flt3L, to first set up Flt3L-DC cultures. Due to a shorter period of cell culture, GMCSF/IL-4 DC cultures were set up 3 days following Flt3L cultures to allow for vaccination with both DC populations to occur on the same day. DCs from Flt3L cultures were harvested on day 9 and depleted of B220 $0^{+}$pDCs. Following depletion, Flt3L-cDCs were placed back into culture and stimulated with Pam3Cys/Poly I:C. GMCSF/IL-4 DCs were stimulated on day 6 of cell culture with LPS/Pam3Cys. Following 24 hours of stimulation, DCs were harvested and loaded with $10 \mu \mathrm{M}$ SIINFEKL peptide for 2 hours. A proportion of Flt3L-cDCs and GMCSF/IL-4 DCs were not loaded with SIINFEKL as a control. Following incubation, both DC populations 
were washed to remove unbound peptide and DCs were titrated. C57BL/6 mice were vaccinated with various numbers of Flt3L or GMCSF/IL-4 DCs, as indicated in Figure 4.6. All mice were tumour challenged with $1 \times 10^{5}$ B16.OVA melanoma cells 7 days following vaccination.

As this experiment aimed to study the ability of the two different DC populations to induce a $\mathrm{CD}^{+} \mathrm{T}$ cell response and consequently tumour rejection, DCs were loaded with SIINFEKL peptide, the OVA epitope recognised by $\mathrm{CD} 8^{+} \mathrm{T}$ cells. While whole OVA protein would have the advantage of providing both a $\mathrm{CD} 4^{+}$and $\mathrm{CD} 8^{+} \mathrm{T}$ cell response, potential differences in protein uptake and processing between Flt3L-cDCs and GMCSF/IL-4 DCs would complicate interpretation of results. Therefore, SIINFEKL peptide was chosen to achieve comparable antigen loading onto both DC types. This made it possible to assess the efficacy of the injected DC vaccine to induce tumour protection, via induction of $\mathrm{CD}^{+} \mathrm{T}$ cell response.

Compared to the tumour only control, mice that were vaccinated with varying numbers of Flt3L-cDCs or GMCSF/IL-4 DCs showed delayed tumour growth (Figure 4.7B and 4.7C). Vaccination with $1 \times 10^{5}$ Flt3L-cDCs conferred up to 80 percent protection in mice. In addition, the single mouse that had developed a tumour showed a delay in tumour onset compared to the tumour only controls (Figure 4.7B). Vaccination with $1 \times 10^{4}$ or $1 \times 10^{3}$ Flt3L-cDCs also induced some tumour protection in mice, but these doses were less effective compared to vaccination with $1 \times 10^{5}$ Flt3L-cDCs. In contrast to Flt3L-cDCs, mice that were vaccinated with $1 \times 10^{5}$ or $1 \times 10^{4} \mathrm{GMCSF} / \mathrm{IL}-4$ were completely protected from tumour growth (Figure 4.7C). Mice that received activated Flt3L-cDCs not loaded with SIINFEKL peptide all grew tumours at a similar rate to the tumour only control (Figure 4.7B), while a slight delay was seen in mice vaccinated GM-CSF/IL-4 DCs not loaded with peptide (Figure 4.7C). However, despite this delay observed, all mice that received either DC population not loaded with SIINFEKL peptide do not survive, thus demonstrating the specificity of the anti-tumour immune response. In summary, vaccination with $1 \times 10^{5}$ Flt3L-cDCs produced the best protective effect, whereas vaccination with $1 \times 10^{5}$ or $1 \times 10^{4}$ GMCSF/IL-4 DCs was equally effective at inducing protection. 
Figure 4.7: Vaccination with graded numbers of Flt3L-cDCs and GMCSF/IL-4 DCs can induce tumour protection in mice. BM cells extracted from C57BL/6 mice were cultured in the presence of Flt3L or GMCSF/IL-4. GMCSF/IL-4 cultures were set up 3 days following Flt3L cultures to allow for vaccination with each DC type to occur on the same day. On day 9 of cell culture, Flt3L-DCs were depleted of B220 $0^{+} \mathrm{pDCs}$, (Chapter 2, (2.4.3.3)), and Flt3L-cDCs were placed back into culture and stimulated with Pam3Cys/Poly I:C, at the following concentrations: Poly I:C $(50 \mu \mathrm{g} / \mathrm{mL})$ and Pam3Cys $(1 \mu \mathrm{g} / \mathrm{mL})$. GMCSF/IL-4 DCs were stimulated on day 6 of cell culture with LPS/Pam3Cys at the following concentrations: LPS $(100 \mathrm{n} / \mathrm{mL})$ and Pam3Cys $(100 \mathrm{ng} / \mathrm{mL})$. Following 24 hours of stimulation, DCs from

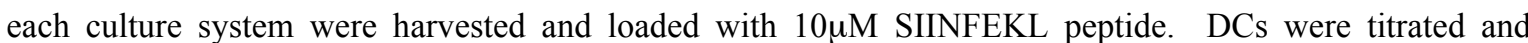
varying numbers were injected s.c. into the right flank of $\mathrm{C} 57 \mathrm{BL} / 6$ mice. All mice were injected s.c. into the left flank with $1 \times 10^{5}$ B16.OVA melanoma cells, 7 days post vaccination. A tumour was scored as positive when it reached $4 \mathrm{~mm}^{2}$. Tumour size was measured with Mitutoyo callipers and mice were euthanized when tumour size reached $150-200 \mathrm{~mm}^{2}$. (A) Timeline showing experimental procedure of tumour experiment. Left panel of (B) and (C) shows mean tumour size up onto the day at which the first mouse within the group was culled due to a large tumour (150-200 $\mathrm{mm}^{2}$ ). Right panel of (B) and (C) shows percentage of survival of mice vaccinated with varying numbers of Flt3L-cDCs and GMCSF/IL-4 DCs, respectively. Tumour size is shown as mean \pm S.D and data are from one of two independent experiments with similar results. Experiment contained 5 mice per group. See Appendix 6 for tumour size data of individual mice. 
A)

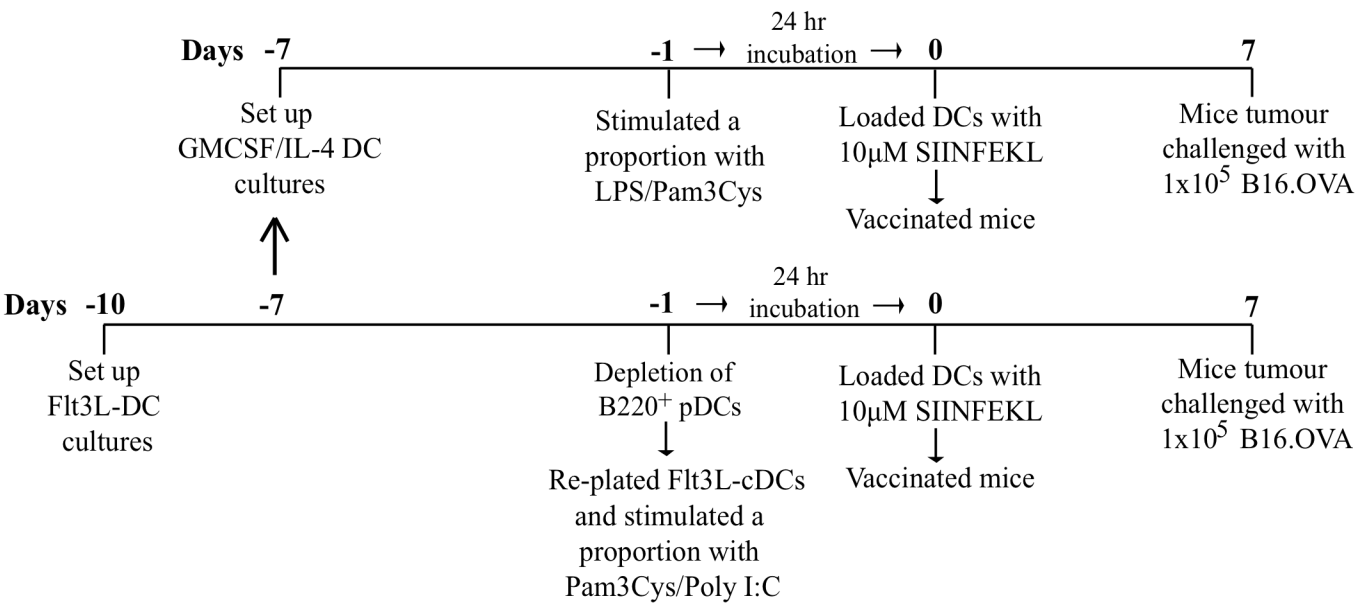

B)

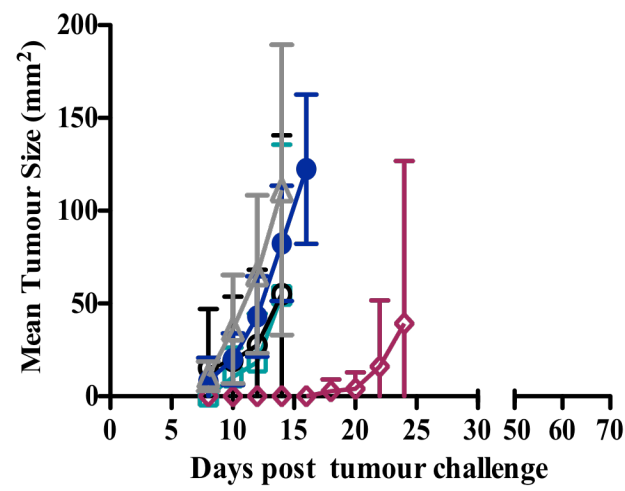

C)

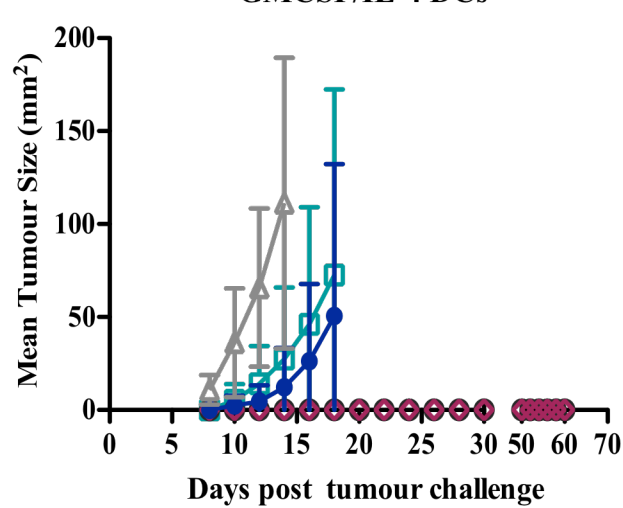

FIt3L-cDCs
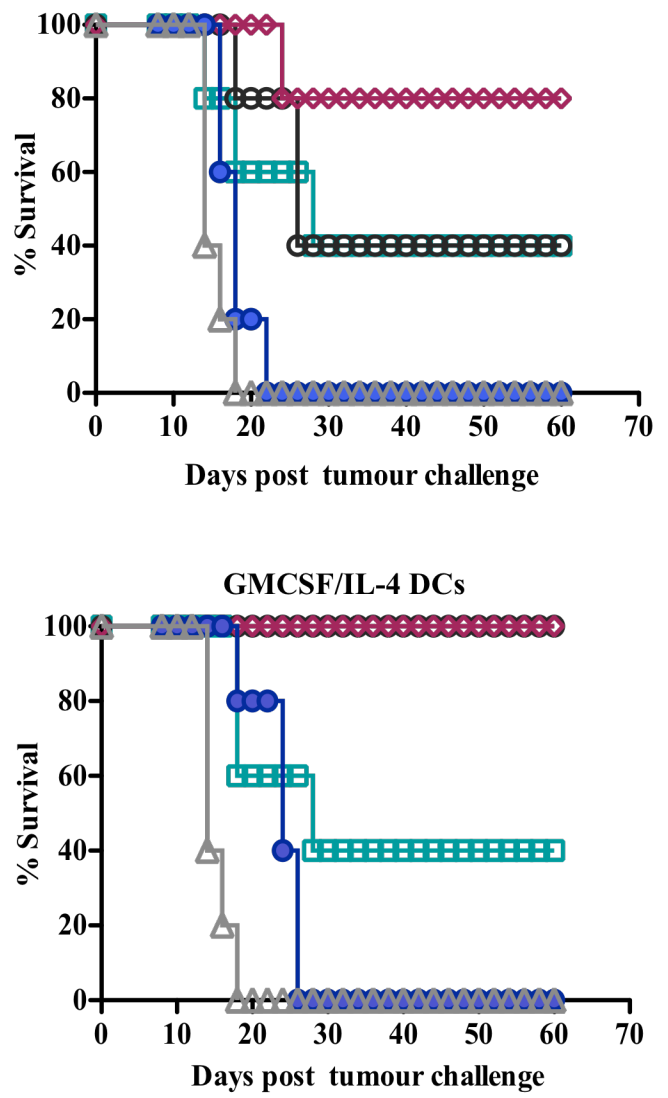

\footnotetext{
$\leftarrow 1 \times 10^{5}$ FIt3L-cDCs or GMCSF/IL-4 DCs (+ SIINFEKL)

- $1 \times 10^{4}$ FIt3L-cDCs or GMCSF/IL-4 DCs (+ SIINFEKL)

๑ $1 \times 10^{3}$ Flt3L-cDCs or GMCSF/IL-4 DCs (+ SIINFEKL)

- $1 \times 10^{5}$ Flt3L-cDCs or GMCSF/IL-4 DCs (No SIINFEKL)

$\triangle$ Tumour only
} 


\subsubsection{Disruption of Flt3L-DC clusters prior to stimulation with TLR agonists does not influence tumour protection}

As mentioned in section 4.3.5, both Flt3L-cDCs and GMCSF/IL-4 DCs were stimulated with optimal TLR agonist combinations, to achieve comparable activation status. However, because Flt3L-DCs were harvested from culture on day 9 and handled for depletion of $\mathrm{B} 220^{+} \mathrm{pDCs}$, there was a possibility of Flt3L-cDCs becoming activated due to this process. In consequence, Flt3L-cDCs maybe more activated than GMCSF/IL-4 DCs following TLR stimulation, which can influence the ensuing anti-tumour immune response. Therefore, we vaccinated mice with total Flt3L-DCs to compare their efficacy to Flt3L-cDCs.

BM cells were cultured in the presence of Flt3L for 9 days to generate Flt3L-DCs. On day 9, a select proportion of Flt3L-DCs was harvested, depleted of $\mathrm{B} 220^{+} \mathrm{pDCs}$ (Chapter 2, (2.4.3.3)) and Flt3L-cDCs were placed back into culture. Flt3L-DC cultures not intended for depletion of $\mathrm{B} 220^{+} \mathrm{pDCs}$ remained in culture and were stimulated at the same time as the depleted population with Pam3Cys/Poly I:C. DCs were harvested, 24 hours following

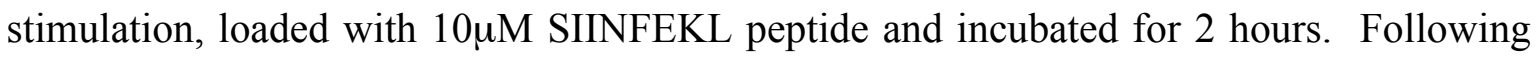
incubation, DCs were washed to remove unbound peptide and $1 \times 10^{5}$ total Flt3L-DCs or $1 \times 10^{5}$ Flt3L-cDCs were injected into C57BL/6 mice. Mice were challenged with $1 \times 10^{5}$ B16.OVA melanoma cells 7 days following vaccination.

From our results, we found that mice were completely protected from tumour growth following vaccination with Flt3L-DCs that were either depleted or not depleted of B220 ${ }^{+}$ pDCs (Figure 4.8). Therefore, it does not appear that B220 ${ }^{+}$pDC depletion influences the protective ability of Flt3L-cDCs, as tumour protection was the same in both vaccination groups. 

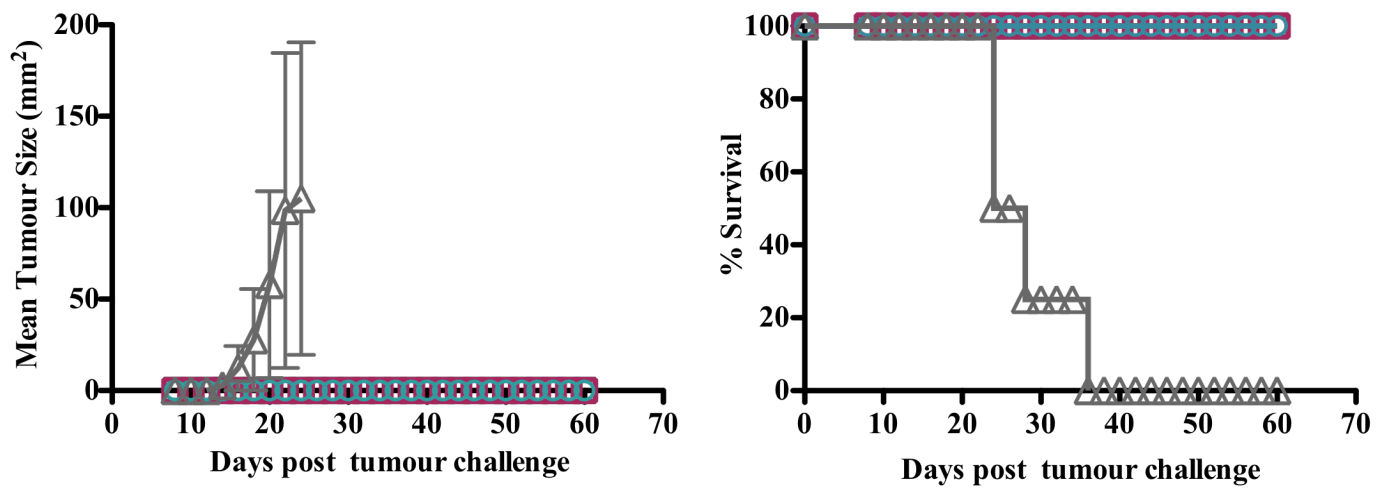

$$
\begin{aligned}
& Ð 1 \times 10^{5} \text { Flt3L-cDCs } \\
& \ominus 1 \times 10^{5} \text { Flt3L-DCs (not depeleted of pDCs) } \\
& \pm \text { Tumour only }
\end{aligned}
$$

Figure 4.8: Vaccination with Flt3L-DCs or Flt3L-cDCs induces the same level of tumour protection. BM cells extracted from $\mathrm{C} 57 \mathrm{BL} / 6$ mice were cultured in the presence of Flt3L for 9 days. On day 9 of cell culture, a selected proportion of Flt3L-DCs were harvested and depleted of B220 pDCs (Chapter 2, (2.4.3.3)). Following depletion, Flt3L-cDCs were placed back into culture and stimulated with Pam3Cys/Poly I:C at the following concentrations: Poly I:C $(50 \mu \mathrm{g} / \mathrm{mL})$ and Pam3Cys $(1 \mu \mathrm{g} / \mathrm{mL})$. Flt3L-DCs that were not depleted of $\mathrm{B} 220^{+} \mathrm{pDCs}$ were stimulated simultaneously. Following 24 hours of stimulation, DCs from each group were harvested and loaded with 10uM SIINFEKL peptide. DCs were resuspended at the appropriate concentration and $1 \times 10^{5}$ Flt3L-cDCs or $1 \times 10^{5}$ total Flt3L-DCs were injected s.c. into the right flank of $\mathrm{C} 57 \mathrm{BL} / 6$ mice. 7 days post vaccination, all mice were injected s.c. into the left flank with $1 \times 10^{5}$ B16.OVA melanoma cells. A tumour was scored as positive when it reached $4 \mathrm{~mm}^{2}$ and was measured with Mitutoyo callipers. Mice were euthanized when tumour size reached $150-200 \mathrm{~mm}^{2}$. Left panel shows mean tumour size up onto the day at which the first mouse within the group was culled due to a large tumour $\left(150-200 \mathrm{~mm}^{2}\right)$. Right panel shows percentage of survival of mice vaccinated with either Flt3L-cDCs or Flt3L-DCs. Tumour size is shown as mean \pm S.D and data are from one of two independent experiments with similar results. Experiment contained 5 mice per group.

\subsubsection{Unstimulated and stimulated Flt3L-cDCs exhibit similar levels of tumour protection}

Studies have shown that DC maturation is key to the induction of an effective anti-tumour immune response (248). Therefore, we determined the relative contribution of combined TLR ligation on the ability of Flt3L-cDCs to protect mice from tumour growth by direct comparison to vaccination with unstimulated Flt3L-cDCs. Furthermore, mice were also 
vaccinated with GMCSF/IL-4 DCs to compare the efficacy to Flt3L-cDCs. We choose to vaccinate mice with $1 \times 10^{5}$ Flt3L-cDCs, which were observed to induce maximal tumour protection in mice. As it was observed that vaccination with $1 \times 10^{4}$ GMCSF/IL-4 DCs worked as well as vaccination with $1 \times 10^{5}$, we chose a lower dose of $1 \times 10^{4}$ GMCSF/IL-4 DCs for vaccination (Figure 4.7C).

As outlined in Figure 4.9A, Flt3L-DC cultures were set up 3 days prior to GMCSF/IL-4 DC cultures to allow for vaccination with both DC populations to occur on the same day. DCs from Flt3L cultures were harvested on day 9 and depleted of B220 $0^{+}$DCs (Chapter 2, (2.4.3.3)). Flt3L-cDCs were placed back into culture and a selected proportion were stimulated with Pam3Cys and Poly I:C, for 24 hours. A proportion of GMCSF/IL-4 DCs were stimulated simultaneously with LPS/Pam3Cys, for 24 hours. Stimulated and unstimulated DCs from both culture systems were harvested, loaded with $10 \mu \mathrm{M}$ SIINFEKL peptide or not loaded as a control. Flt3L-cDC or GMCSF/IL-4 DCs from all treatment groups were washed to remove unbound peptide and resuspended at the appropriate concentrations. C57BL/6 mice were vaccinated with $1 \times 10^{5}$ Flt3L or $1 \times 10^{4}$ GMCSF/IL-4 DCs, as indicated in Figure 4.9A. All mice were tumour challenged with $1 \times 10^{5}$ B16.OVA melanoma cells, 7 days following vaccination.

Vaccination with $1 \times 10^{5}$ Flt3L-cDCs or $1 \times 10^{4}$ GMCSF/IL-4 DCs, stimulated with the optimal TLR agonist combination and loaded with SIINFEKL, induced complete protection from tumour growth (Figure 4.9B, 4.9C, respectively). Therefore, with respect to vaccination with $1 \times 10^{5}$ Flt3L-cDCs, a slight variation in percentage of survival was seen within this experiment compared to those previously observed (Figure 4.7B), however, the same result was reproduced for GMCSF/IL-4 DCs. We also found that unstimulated Flt3L-cDCs or GMCSF/IL-4 DCs, loaded with SIINFEKL, conferred up to 80 or 60 percent tumour protection, respectively (Figure 4.9B, 4.9C, respectively). Furthermore, unstimulated Flt3L-cDCs, not loaded with SIINFEKL were also able to elicit some protective effect, conferring up to 40 percent protection in mice. In summary, both Flt3LcDCs and GMCSF/IL-4 DCs, in the unstimulated state maintain some protective ability, but only stimulation with TLR agonists can induce complete protection from tumour growth. 
Figure 4.9: Stimulated and unstimulated Fit3L-cDCs do not differ substantially in their ability to protect mice from tumour growth. Flt3L-DC cultures were set up, followed by GMCSF/IL-4 DC cultures to allow for vaccination with each DC type to occur on the same day. On day 9 of cell culture, Flt3L-DCs were depleted of B220 $0^{+}$pDCs (Chapter 2, (2.4.3.3)), placed back into culture and a proportion was stimulated with Pam3Cys/Poly I:C at the following concentrations: Poly I:C (50 $\mathrm{gg} / \mathrm{mL})$ and Pam3Cys $(1 \mu \mathrm{g} / \mathrm{mL})$. GMCSF/IL-4 DCs were stimulated on day 6 of cell culture with LPS/Pam3Cys at the following concentrations: LPS (100ng/mL) and Pam3Cys (100ng/mL). Following 24 hours of incubation, stimulated and unstimulated DCs from each culture were harvested, and a selected proportion from each group were loaded with $10 \mu \mathrm{M}$ SIINFEKL peptide and incubated for 2 hours. Following incubation, DC groups loaded with SIINFEKL were washed to remove unbound peptide and $1 \times 10^{5}$ Flt3L-cDCs or $1 \times 10^{4}$ GMCSF/IL-4 DCs were injected s.c. into the right flank of C57BL/6 mice. 7 days following vaccination, $1 \times 10^{5}$ B16.OVA tumour cells were injected s.c. into the left flank of all mice. Tumour growth was scored as positive when it reached $4 \mathrm{~mm}^{2}$. Tumour size was measured with Mitutoyo callipers and mice were euthanized when tumour size reached $150-200 \mathrm{~mm}^{2}$. (A) Timeline depicts experimental procedure of tumour experiment. Left panel of (B) and (C) shows mean tumour size up onto the day at which the first mouse within the group was culled due to a large tumour $\left(150-200 \mathrm{~mm}^{2}\right)$. Right panel of (B) and (C) shows percentage of survival of mice vaccinated with stimulated or unstimulated Flt3L-cDCs or GMCSF/IL-4 DCs. Tumour size is shown as mean \pm S.D and data are from one of 2 independent experiments with similar results. Experiment contained 5 mice per group. See Appendix 7 for tumour size data of individual mice. 
A)

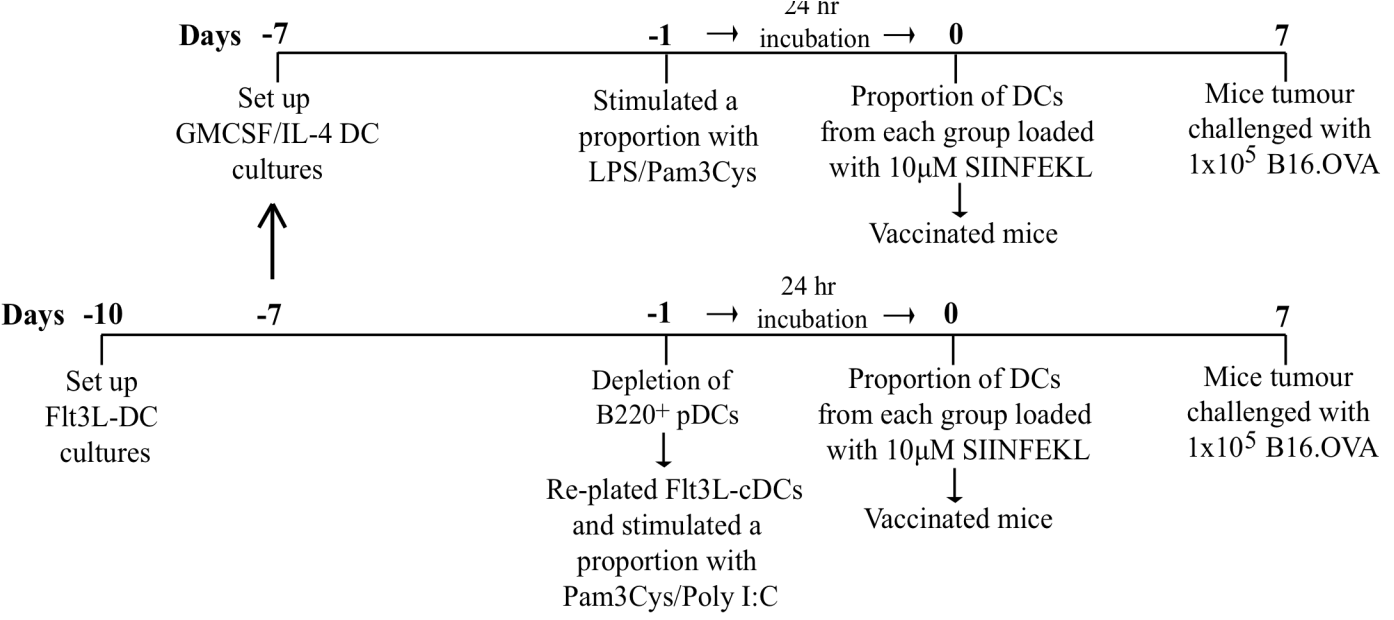

B)

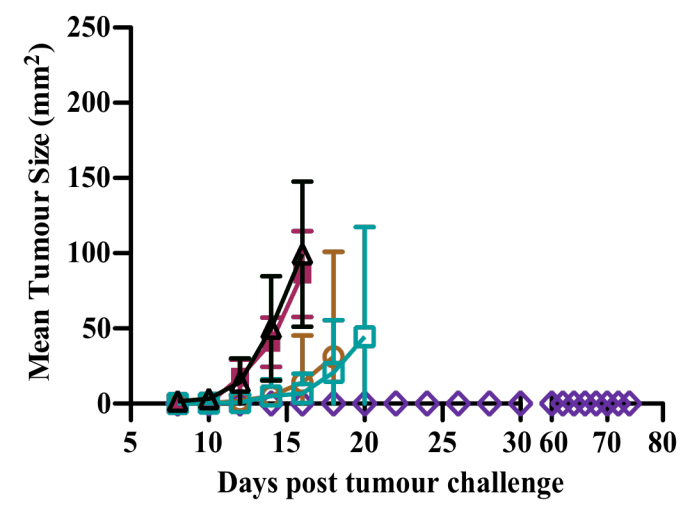

C)

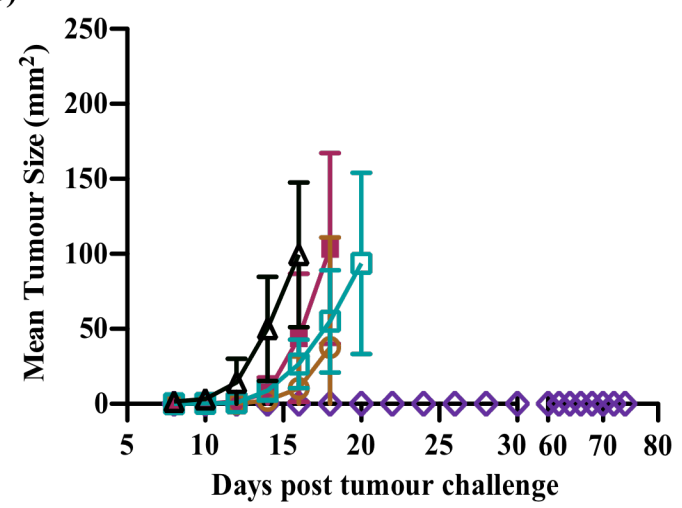

Flt3L-cDCs
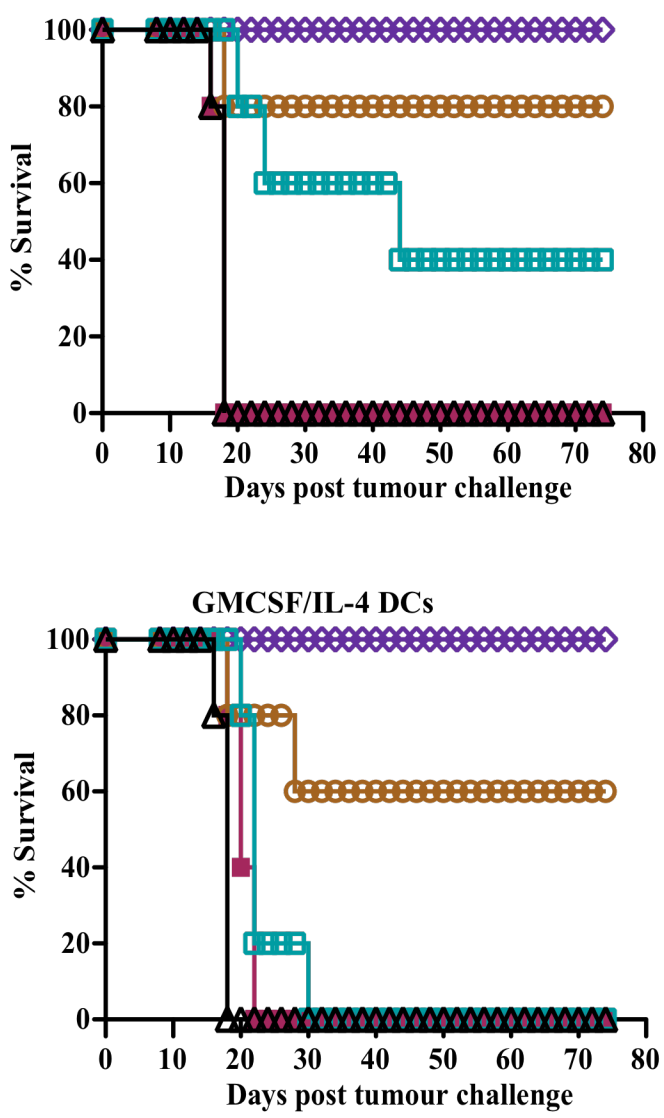

\footnotetext{
$\multimap$ Stimulated + SIINFEKL

$\rightarrow$ Stimulated + No SIINFEKL

$\rightarrow$ Unstimulated + SIINFEKL

\& Unstimulated + No SIINFEKL

$\leftarrow$ Tumour only
} 


\subsection{Discussion}

\subsubsection{Cytokine secretion profiles of Fit3L-DCs following stimulation with the individual or combined TLR agonists.}

The experiments presented within this chapter investigated the functional capability of Flt3L-DCs. Firstly, we determined the relative ability of Flt3L-DCs to secrete both the inflammatory cytokine IL-12 and the anti-inflammatory IL-10, in response to stimulation with individual TLR agonists. In agreement with data published by Naik et al and Brawand et al $(225,233)$, we found that Flt3L-DCs secreted both IL-12p70 and IL-10, following TLR ligation (Figure 4.1). Specifically, each agonist induced a different response with respect to the quantity of IL-12p70 and IL-10 secretion. Furthermore, a trend was observed whereby each agonist induced more secretion of one cytokine than the other, but never similar amounts of both. Overall, this result is indicative of the fact that cytokine production from Flt3L-DCs is regulated by the type of microbial compound that induces activation. Moreover, our cytokine data also indicated that not only could Flt3LDCs induce an inflammatory response through secretion of IL-12, but can also dampen inflammation via production of IL-10. IL-12 is essential for the control of malignancy by virtue of its ability to stimulate both innate and adaptive immune effector cells (281), however, its production can be suppressed by IL-10 (282). Thus, IL-10 production could be a regulatory mechanism to prevent tissue damage from excessive inflammation or possibly autoimmunity. This hypothesis could be investigated in future experiments using intracellular staining to study the kinetics of IL-10 production, which can establish if IL-10 is produced sometime following production of IL-12p70. Moreover, intracellular staining would allow for assessment of which Flt3L-DC subset is producing the regulatory cytokine.

We found that only selected combinations of TLR agonists could enhance the production of pro-inflammatory cytokines, compared to stimulation with the respective agonists. Specifically, enhanced production of IL-12p70 was only observed with combinations of Pam3Cys/Poly I:C or CpG/Poly I:C. Only stimulation with Pam3Cys/Poly I:C was observed to enhance TNF- $\alpha$ production (Figure 4.2), and although there were some variability in this response, this observation was further supported by data derived from 
intracellular staining (discussed in section 4.4.2). The limited response in cytokine secretion following combined TLR ligation is unlike our previous finding with respect to analysis of activation markers. As shown in Chapter 3, we observed that all TLR agonist combinations that induced signalling of both the MyD88-dependent and independent pathways, enhanced expression of either CD40, CD80 or both cDCs and pDCs (Chapter 3, Figure 3.8). Therefore, data gathered throughout this thesis indicated that while many TLR agonist combinations can amplify expression of activation markers on Flt3L-DCs, enhanced production of pro-inflammatory cytokines is highly specific, in that only selected agonist combinations are capable of inducing such events. Furthermore, we also note that those TLR agonist combinations that enhanced secretion of IL-12p70 from Flt3L-DCs, did not induce a 20 to 50 fold increase as previously demonstrated by Napolitani et al, with GM-CSF DCs (235). Instead, we only observed an increase of IL-12p70 production by only 2 fold or less. Given that Napolitani et al, described their result with GM-CSF DCs as synergistic activation, our cytokine data did not indicate a synergistic response. Rather, our data would suggest that combined TLR ligation on Flt3L-DCs serves to induce an additive or sub-additive effect, as opposed to synergistic activation.

\subsubsection{Differential capacity of cytokine secretion from FIt3L-DC subsets}

Kinetics of cytokine secretion from Flt3L-DCs were assessed by intracellular staining, and we identified the $\mathrm{CD} 24^{\text {high }}$ and $\mathrm{CD} 11 \mathrm{~b}^{\text {high }} \mathrm{cDCs}$ as the primary subsets producing proinflammatory cytokines. We have shown that production of TNF- $\alpha$ was only detected within the $\mathrm{CD} 24^{\text {high }} \mathrm{cDCs}$ at 6 hours, following stimulation. Furthermore, we observed that stimulation with Pam3Cys/Poly I:C resulted in an increase in percentage of TNF- $\alpha$ production within the $\mathrm{CD} 24^{\text {high }} \mathrm{cDCs}$. This result supported our initial observation from the biolpex data, where we observed enhanced secretion of TNF- $\alpha$ from Flt3L-DCs stimulated with Pam3Cys/Poly I:C (Figure 4.2).

We have also shown that IL-12p40 production by both CD24 ${ }^{\text {high }}$ and CD11b ${ }^{\text {high }}$ cDCs was detected at both 6 hours and 24 hours after stimulation with Pam3Cys or Pam3Cys/Poly I:C. However, CD11b ${ }^{\text {high }}$ cDCs stimulated with Poly I:C did not exhibit production of IL- 
12 p40 when analysed at 6 hours, but only at 24 hours. Expression of TLR 3 on CD $11 b^{\text {high }}$ cDCs, the receptor which responds to Poly I:C, has been shown to be expressed at very low and undetectable levels by real-time PCR (225). Therefore, given the minimal expression of TLR3, this observation could be the result of the CD11 ${ }^{\text {high }}$ cDCs requiring an extended period of stimulation with Poly I:C in order to acquire sufficient signal to induce their activation and subsequent cytokine production. Thus, this could explain why IL-12p40 production could only be detected at 24 hours. Alternatively, this delay in cytokine production may be the result of Poly I:C interacting with other receptors expressed on CD11b ${ }^{\text {high }}$ cDCs. Other than TLR3, studies have shown that Poly I:C can also be recognised by the receptors RIG-1 and MDA-5 (283). These are sensors of viral RNA and belong to a family of RIG-1-like receptors (RLRs), which are cytoplasmic helicases (284). Both RIG-1 and MDA-5 have been shown to be predominantly expressed on CD8 ${ }^{-}$cDCs (285). Signalling through RIG-1 and MDA-5 induces recruitment of adaptor molecules such as IPS-1, which are distinct to those of TLR mediated signalling, but result in induction of the same transcription factors - namely IRFs and NF- $\kappa \mathrm{B}-$ as TLR3 signalling (284). Therefore, the kinetics of this signalling pathway could be different from TLR mediated Poly I:C recognition. Expression of RIG-1 and MDA-5 on $\mathrm{CD}^{-}$cDCs indicates that they can also be expressed on $\mathrm{CD} 11 \mathrm{~b}^{\text {high }} \mathrm{cDCs}$, which are the CD8 ${ }^{-}$cDCs equivalents (225). Therefore, Poly I:C could be recognized by RIG-1 or MDA-5 expressed on CD $11 b^{\text {high }} \mathrm{cDCs}$ and through this different signalling cascade induce their activation and subsequent secretion of IL-12p40 at a later time point.

pDCs were not observed to produce any IL-12p40 or TNF- $\alpha$ following stimulation with Pam3Cys/Poly I:C, or the respective individual agonists. This is not surprising, as pDCs are known to express low levels of TLR2 and 3, the receptors for Pam3Cys amd Poly I:C, respectively. Conversely, pDCs display high levels of TLR9 and thus primarily respond to activation with its corresponding ligand $\mathrm{CpG}$ (233). Stimulation with $\mathrm{CpG}$ has also been shown to induce production of IL-2p40 from pDCs, but not secretion of bioactive IL12 p70 (225). Furthermore, pDCs reportedly can secrete TNF- $\alpha$ following stimulation with a TLR7 or TLR9 agonist (286). Taken together, these data highlight the fact that cytokine production in response to TLR engagement depends on TLR expression on the different DC subsets and the TLR agonist used to induce activation. 


\subsubsection{Enhanced proliferation of $\mathrm{CD}^{+} \mathrm{T}$ cells by Flt3L-cDCs stimulated with the combined TLR agonists}

The capacity for Flt3L-cDCs to present peptide antigen and induce the activation and proliferation of naïve $\mathrm{CD}^{+}$and $\mathrm{CD}^{+} \mathrm{T}$ cells was also investigated in this chapter. For these experiments, Flt3L-cDCs were either activated with Pam3Cys/Poly I:C or the respective individual TLR agonists, thus effects of combined TLR ligation on T cell responses was also examined (section 4.3.3)

Flt3L-cDCs, stimulated with the combined or individual agonist, induced the proliferation of both $\mathrm{CD}^{+}$and $\mathrm{CD}^{+} \mathrm{T}$ cells in vitro, thus displaying the capacity of antigen presentation. Furthermore, we observed that Flt3L-cDCs stimulated with the combined TLR agonists, were more potent at inducing $\mathrm{CD}^{+} \mathrm{T}$ cell proliferation compared to those stimulated with the individual agonist. Conversely, $\mathrm{CD}^{+} \mathrm{T}$ cell proliferation appeared similar regardless of stimulation with combined or individual TLR agonists (Figure 4.5). It is known that activation of $\mathrm{T}$ cells requires at least 2 signals that is delivered by the APC. Antigen presentation by DCs provides the first signal necessary for $\mathrm{T}$ cell activation. However, $\mathrm{T}$ cells also require a second signal, delivered by the co-stimulatory molecules on the APC (287). As shown in Chapter 3, stimulation with Pam3Cys/Poly I:C improved expression of co-stimulatory molecules on Flt3L-cDCs (Section 3.5.5, Figure 3.8). Therefore, delivery of more co-stimulation by Flt3L-cDCs activated with the combined TLR agonists, may account, at least in part, for the enhanced proliferation of $\mathrm{CD}^{+}$cells observed.

A failure in observing any difference in $\mathrm{CD}^{+} \mathrm{T}$ cell proliferation between the agonists treated groups could be due to high concentration of the antigen (SIINFEKL) being used, which could induce a maximal proliferative response. Therefore, it would be ideal to perform a future $\mathrm{T}$ cell proliferation assay using only a fixed number of DCs and titrating the antigen concentration. Any potential differences in $\mathrm{CD}^{+} \mathrm{T}$ cell proliferation between the combined and single TLR stimulation may therefore be observed. 


\subsubsection{Enhanced cross-presentation of soluble antigen by Flt3L-cDCs stimulated with the combined TLR agonists}

DCs are capable of taking up, processing and cross-presenting exogenous antigen to CD ${ }^{+}$ T cells. Using soluble OVA as a model of exogenous antigen, we evaluated the crosspresentation capacity of Flt3L-cDCs and the impact of combined or single TLR ligation on this process. We observed that unstimulated Flt3L-cDCs were capable of cross-presenting soluble OVA, but with less efficiency compared to Flt3L-cDCs stimulated with the individual agonists (Figure 4.6B). Moreover, Flt3L-cDCs that were stimulated with the combined TLR agonists exhibited the greatest capacity in cross-presentation compared to unstimulated Flt3L-cDCs or those stimulated with the respective individual agonists (Figure 4.6B).

Delamarre et al showed that immature GM-CSF DCs generated in vitro, can internalise exogenous antigens, but were incapable of cross-presentation. However, upon disruption of DC clusters (by gentle pipetting of the culture media) unsitmulated GM-CSF DCs were able to cross-present soluble OVA (288). Cluster disruption is a stimulus known to induce DC maturation in vitro, however its mechanism is not fully understood (289). It is thought that disruption of cell-cell adhesion that is mediated by E-cadherin, generates a signal that induces maturation (288). Potentially, disaggregation of DC clusters may represent a mechanism in which unstimulated Flt3L-cDCs acquire the capability for crosspresentation. In our cross-presentation assay, Flt3L-DCs were harvested on day 9 for $\mathrm{B} 220^{+} \mathrm{pDC}$ depletion, however, it was protocol to gently pipette the culture media to detache loosely adherent DCs from the bottom of the wells. Thus, it is possible that unstimulated Flt3L-cDCs acquired maturation signals that were sufficient to induce crosspresentation of OVA. Alternatively, cross-presentation by unstimulated Flt3L-cDCs could be an artefact of high antigen concentration within the culture, which could induce antigen processing and cross-presentation.

We have shown that the differential capacity in cross-presentation between Flt3L-cDCs stimulated with the combined or single TLR agonists was not associated with differences in expression of co-stimulatory molecules, as presentation of SIINFEKL peptide was similar in all cases (Figure 4.7C). Thus, our data suggested that stimulation with 
Pam3Cys/Poly I:C promoted enhanced cross-presentation by acting on the intracellular cross-presentation mechanisms. In a cross-presentation assay using Flt3L-DCs generated in vitro, de Brito et al were able to show using soluble OVA that stimulation with $\mathrm{CpG}$ significantly delayed antigen degradation compared to those of untreated Flt3L-DCs (290). Reportedly, delayed antigen degradation is a mechanism which favours cross-presentation (291). Furthermore, de Brito et al showed that OVA accumulated at high densities in CpG-treated DCs. Therefore, these are potential mechanisms that increase the efficacy of Flt3L-cDCs to cross-present OVA following stimulation with the individual agonists, and which can be enhanced by combined TLR stimulation.

\subsubsection{Induction of anti-tumour immune response by Fit3L-cDCs}

As a final point of investigation within this thesis, we determined the efficacy of Flt3LcDCs, as immunotherapy against cancer. In a mouse tumour model using B16.OVA melanoma cells, we compared the anti-tumour activity of Flt3L-cDCs to GMCSF/IL-4 DCs that were both stimulated with optimal TLR agonist combinations.

In our initial experiment, we sought to determine the number of Flt3L-cDCs or GMCSF/IL-4 DCs that will induce maximal protection from tumour growth. We observed that vaccination with just $1 \times 10^{4}$ GMCSF/IL-4 DCs was sufficient to induce complete tumour protection in mice, while $1 \times 10^{5}$ Flt3L-cDCs was required to produce a similar outcome, with 80 percent protection (Figure 4.7B, 4.7C). Given that a lower dose of GMCSF/IL-4 DCs can completely protect mice from tumour growth, it is apparent these DCs display a higher efficacy at inducing anti-tumour immune responses, compared to Flt3L-cDCs.

DC maturation is a critical parameter in the induction of anti-tumour immune responses. This has been shown within several clinical trials, whereby injection with immature MoDCs into melanoma patients, did not lead to significant B and $\mathrm{T}$ cell responses compared to matured DCs $(138,292)$. Thus, given the importance of DC maturation on the ensuing anti-tumour immune response, it was meaningful to determine the impact of stimulation with the combined TLR agonists on the ability of Flt3L-cDCs and GMCSF/IL-4 DCs to 
protect mice from tumour growth. Vaccination with matured and activated GMCS/IL-4 DCs or Flt3L-cDCs, loaded with SIINFEKL, was required for complete tumour protection. However, we also observed that vaccination with $1 \times 10^{5}$ unstimulated Flt3L-cDCs or $1 \times 10^{4}$ unstimulated GMCSF/IL-4 DCs, loaded with SIINFEKL, could induce up to 60 and 80 percent tumour protection, respectively (Figure 4.9B, 4.9C). This observation may be due to the high number of DCs chosen for vaccination. Alternatively, the unstimulated DC populations may already have possessed some level of maturation. Although unstimulated Flt3L-cDCs and GMCSF/IL-4 DCs were induced to mature with TLR agonists, it is likely that handling of these DCs in order to prepare them for vaccination could have induced some activation. Thus, given that these DCs may not be entirely non-activated, antitumour immune responses can be elicited if a considerable number of DCs is administered into the mice. Therefore, future experiments could focus on vaccination with titrated numbers of unstimulated Flt3L-cDCs and GMCSF/IL-4 DCs to confirm if lower DC numbers could results in less tumour protection. Preferably, this would be performed alongside stimulated Flt3L-cDCs and GMCSF/IL-4 DCs, also titrated in the same manner as the unstimulated DCs for a direct comparison.

\subsection{Conclusion}

Within this chapter, we have shown that TLR stimulation could induce Flt3L-DCs to acquire functional characteristics that are important for their application in immunotherapy. Specifically, we found that stimulation with individual TLR agonists induced Flt3L-DCs to secrete both pro-inflammatory and anti-inflammatory cytokines. Production of pro-inflammatory cytokines could be further enhanced by stimulation with combined TLR agonists, however, this response was highly restricted to few agonists, with only the combination of Pam3Cys/Poly I:C enhancing secretion of the cytokines IL-12p70 and TNF- $\alpha$. Furthermore, not only could this combination enhance cytokine production, but Flt3L-cDCs stimulated with Pam3Cys/Poly I:C were better than those stimulated with the respective individual agonists at inducing $\mathrm{CD} 4^{+} \mathrm{T}$ proliferation and cross-presentation of soluble antigen to $\mathrm{CD} 8^{+} \mathrm{T}$ cells. When tested for their ability to confer tumour protection in mice, vaccination with $1 \times 10^{5}$ Flt3L-cDCs was required to induce maximal 
anti-tumour immunity, while as little as $1 \times 10^{4}$ GMCSF/IL-4 DCs could completely protect mice from tumour growth. Therefore, we found that Flt3L-cDCs can be used for immunotherapy, but even under optimal conditions of activation, they display less efficacy compared to GMCSF/IL-4 DCs. 
Chapter 5.

General Discussion 


\subsection{General overview of findings}

DCs are crucial for the generation of immune responses, and as such have been used as therapeutic cancer vaccines. A well established, and commonly used procedure for generating DCs in culture from human blood monocytes involves the culture of these cells with the cytokines GM-CSF and IL-4 $(83,213)$. However, GMCSF/IL-4 DCs do not show the heterogeneity in DC phenotype found within steady-state DCs, but rather share similar properties to DCs that arise in vivo during inflammation. Conversely, the culture of BM cells in Flt3L generates DCs that are phenotypcially distinct from GMCSF/IL-4 DCs and more closely resemble steady-state DCs in vivo $(78,214)$. Therefore, the general aim of this thesis was to determine the applicability of Flt3L-DCs for cancer immunotherapy.

The clinical application of ex vivo generated DCs requires a detailed understanding of their phenotypic and functional properties for optimal immune induction. In Chapter 3, we first characterised the phenotype of Flt3L-DCs before and after stimulation with TLR agonists. We have shown that murine BM cells cultured in Flt3L generated DCs consisting of two primary subsets: $\mathrm{CD} 11 \mathrm{c}^{+} \mathrm{B} 220^{+} \mathrm{pDCs}$ and $\mathrm{CD} 11 \mathrm{c}^{+} \mathrm{B} 220^{-} \mathrm{cDCs}$. The cDCs could be further subdivided into two populations that are CD11 $b^{\text {high }}$ and CD2 $4^{\text {high }}$. This is in line with the DC subsets reported by Naik et al (225), who also described these populations as the equivalents of mouse splenic steady-state DCs. In contrast to Flt3L-DCs, GMCSF/IL4 DCs display a uniform $\mathrm{CD} 24^{\text {low }} \mathrm{CD} 11 \mathrm{~b}{ }^{\text {high }}$ phenotype (214). Furthermore, we also found that these different culture conditions lead to differences in morphology, as we have observed that Flt3L-DCs were smaller in size compared to GMCSF/IL-4 DCs.

Within the context of immunotherapy, the maturation of DCs is essential in order to induce immunity. Comparative studies with immature or matured DCs have shown that only mature DCs are superior inducers of T cell responses (292). One way to induce DC activation in vitro is the use of specific TLR agonists. Our results showed that stimulation with TLR agonists induced Flt3L-DCs to acquire an activated phenotype, as evaluated by the up-regulation of activation markers such as CD80, CD40 and MHC II. Brawand et al showed that DCs generated in GM-CSF cultures need to switch from oxidative phosphorylation to glycolytic metabolism to become activated in response to TLR 
stimulation (245). In light of these findings, we set forth to determine if inhibiting glucose metabolism by treatment with the competitive glycolysis inhibitor 2-DG impairs the maturational capacity of Flt3L-cDCs. Although the results from our experiments are preliminary and require validation, they provide new insight into another level of distinction between Flt3L-cDCs and GMCSF/IL-4 DCs during TLR induced activation. Unlike LPS activated GMCSF/IL-4 DCs, which could not up-regulate activation markers in all 2-DG concentrations used, we found that Flt3L-cDCs had a threshold level, whereby only high concentrations of 2-DG inhibited their maturational capacity. This suggested that Flt3L-cDCs do not switch to glycolytic metabolism to the same extent as GMCSF/IL4 DCs. Further experiments are required in order to confirm whether or not activated Flt3L-cDCs are glycolytic, but our preliminary data suggests that while metabolic reprogramming is a critical control point for the maturation of inflammatory DCs following TLR stimulation, this is not the case for steady-state DCs.

As steady-state DCs consist of different subpopulations, we sought to examine the effect of a variety of TLR agonists on both $\mathrm{cDCs}$ and pDCs. We observed that the $\mathrm{cDC}$ subset responded to a broad range of TLR agonists (LPS, Pam3Cys, Poly I:C and CpG), with no considerable differences seen between these stimuli. In contrast, pDC maturation was induced to the greatest extent by the agonist $\mathrm{CpG}$, whereas other agonists induced a less pronounced response. As previously stated, these differences in response are likely due to the differential expression of TLRs among the DC subsets (Chapter 3, section 3.4.2) (225, 233). Therefore, we were able to show that both $\mathrm{cDCs}$ and pDCs can be induced to acquire an activated phenotype following stimulation with individual agonists, but their maturational response is dictated by the expression of the TLR between the two subsets. Given the heterogeneity of Flt3L-DCs and the different repertoire of TLRs that each subset expresses, our findings highlight the importance of careful selection of TLR agonists that can induce the maturation of cDCs and pDCs. In this respect, the agonist $\mathrm{CpG}$ would appear to be ideal, given that the corresponding receptor TLR9 is expressed on all Flt3LDC types (225). Alternatively, one could use a cocktail of agonists, to induce activation of all three subsets. This could have the benefit of enhancing the activation of individual DC subtypes, as it has been shown that the combined triggering of MyD88-dependent and independent (TRIF) signalling pathways can induce a synergistic effect on DCs (236). 
While this has been shown with GM-CSF DCs (236), the possibility of synergistic activation has not been investigated with Flt3L-DCs.

In this thesis we found that the combined activation of MyD88-dependent and independent pathways can improve the up-regulation of activation markers on Flt3L-DCs. Analysis of the effects from combined TLR stimulation was further extended in Chapter 4, by determining if enhanced activation marker expression correlated with increased production of pro-inflammatory cytokines. While many combinations were found to increase expression of activation markers, only combinations of Pam3Cys/Poly I:C and CpG/Poly $\mathrm{I}: \mathrm{C}$ induced higher secretion of IL-12p70. In addition, Pam3Cys/Poly I:C were shown to enhance secretion of TNF- $\alpha$, which was why this combination was deemed optimal. Overall, our finding implicates that the analysis of surface phenotype cannot be used as surrogate marker for functional properties, such as cytokine secretion. Furthermore, although we found that specific combinations of TLR agonists can enhance cytokine secretion, this was only seen in an additive manner and not synergistically.

In Chapter 4, it was also found that Flt3L-cDCs that were stimulated with Pam3Cys/Poly $\mathrm{I}: \mathrm{C}$ were better at inducing $\mathrm{T}$ cell proliferation compared to Flt3L-cDCs that were stimulated with the respective individual TLR agonists. Specifically, this was seen in enhanced proliferation of $\mathrm{CD}^{+} \mathrm{T}$ cells by direct presentation of peptide antigen and of $\mathrm{CD}^{+} \mathrm{T}$ cells by cross-presentation. Taken together, we found that stimulation with combined TLR agonists can improve Flt3L-DC activation and function. This approach can be used as the basis for developing effective Flt3L-DC vaccines.

One of the aims of this thesis was to determine the ability of Flt3L-DCs to confer tumour protection in vivo. Flt3L-cDCs were either left untreated or optimally activated with Pam3Cys/Poly I:C and their ability to induce tumour protection was compared to GMCSF/IL-4 DCs, equally unstimulated or activated with LPS/Pam3Cys. Even unstimulated DCs were able to prevent tumour formation. However, complete tumour protection was only observed if optimally activated DCs were used. Under these conditions, GMCSF/IL-4 DCs were about 10-fold more effective than Flt3L-cDCs. 
In conclusion, Flt3L-DC vaccines effectively induce immune responses in vivo and therefore can be used as the basis of cancer immunotherapies. However, the results from the tumour studies in this thesis suggest that Flt3L-cDCs are not superior to GMCSF/IL-4 DCs if used as a prophylactic vaccine.

\subsection{Future directions}

As we have shown that Flt3L-cDCs can confer tumour protection in mice (Chapter 4), the following represents a list of suggestions for future work to establish mechanisms, which contribute to the ability of Flt3L-cDCs to induce anti-tumour immunity in vivo. While similar experiments have been performed by other authors with Flt3L-DCs $(214,233$, 234), these future experiments will provide new information with respect to demonstrating the efficacy of Flt3L-DCs that have been optimally activated with Pam3Cys/Poly I:C. All of the following experiments should be performed alongside optimally activated GMCSF/IL-4 DCs for comparison.

- Induction of immune responses requires migration of the administered DCs to the draining $\mathrm{LN}$ for antigen presentation to naïve $\mathrm{T}$ cells. Thus, it would be ideal to confirm the migratory ability of Flt3L-cDCs following vaccination. For this experiment, Flt3L-cDCs would be stimulated with Pam3Cys/Poly I:C or the respective individual agonists, and subsequently labelled with a fluorescent dye such as CFSE. Flt3L-cDCs would then be injected into C57BL/6 mice s.c and the draining LN can then be extracted 24 hours post injection. Cells from the LN can then be analysed by flow cytometry for expression of CFSE.

- Migration of DCs to the draining LN requires expression of the chemokine receptors CCR7 $(274,293)$ and CXCR4 (294). Thus, if Flt3L-cDCs, activated with Pam3Cys/Poly I:C exhibit enhanced migratory ability compared to those activated with the individual TLR agonists, chemokine receptor expression should be analysed to confirm if their superior migratory ability is due to enhanced expression of chemokine receptors. One method of determining chemokine 
receptor expression on DCs involves fluorescent antibody labelling for CCR7 and CXCR4, and analysis by flow cytometry.

- In this thesis, Flt3L-cDCs were loaded with SIINFEKL (OVA epitope recognised by $\mathrm{CD}^{+} \mathrm{T}$ cells) prior to vaccination. Thus, it was expected that Flt3L-cDCs would induce an antigen-specific $\mathrm{CD}^{+} \mathrm{T}$ cell response in vivo, driving anti-tumour immunity. To confirm if Flt3L-cDCs are inducing $\mathrm{CD}^{+} \mathrm{T}$ cell activation and proliferation, an in vivo $\mathrm{T}$ cell proliferation assay could be performed. For this assay, $\mathrm{CD}^{+}$transgenic $\mathrm{T}$ cells (specific for SIINFEKL) would be labelled with the fluorescent dye CFSE and then adoptively transferred i.v. into C57BL/6 mice. Flt3L-cDCs stimulated with Pam3Cys/Poly I:C or the respective individual agonists would be loaded with SIINFEKL are then injected into mice, 24 hours post $\mathrm{T}$ cell adoptive transfer. Three days later, draining $\mathrm{LN}$ from mice could be excised and CFSE labelled $\mathrm{CD} 8^{+} \mathrm{T}$ cells are examined for CFSE dilution by flow cytometry. In parallel to analysing the division profile of the transferred $\mathrm{CD} 8^{+} \mathrm{T}$ cells, expression of $\mathrm{T}$ cell activation markers could also be assessed. Markers that are known to be up-regulated on activated $\mathrm{CD}^{+} \mathrm{T}$ cells include CD69 (187) and CD44 (234).

- For tumour killing to occur, $\mathrm{CD}^{+} \mathrm{T}$ cells that are activated by Flt3L-cDCs must exhibit cytotoxic activity. The cytotoxic capacity of $\mathrm{CD}^{+} \mathrm{T}$ cells that have been primed with Flt3L-cDCs activated with the combined or individual TLR agonists can be assayed in vivo against splenocyte target cells (234).

Further experiments are required in order to confirm if metabolism of Flt3L-cDCs is skewed towards aerobic glycolysis following activation, as shown with GM-CSF DCs (245)

- A western blot could be performed with Flt3L-cDCs to see whether there would be an increase in expression of glucose transporters (GLUT1) following activation, which has been shown to be increased on activated GM-CSF DCs. Analysis of glucose consumption and lactate production would also provide supporting 
evidence for whether Flt3L-cDCs are glycolytic during activation. Furthermore, oxygen consumption should also be assessed, as changes in rate of consumption between non-activated and activated Flt3L-cDCs could also indicate a switch to glycolysis.

\subsection{Final conclusion}

The findings of this thesis show that Flt3L-DCs are phenotypically different from GMCSF/IL-4 DCs and do not form a homogenous population but can be divided into three subsets. Flt3L-DC subsets differ in their response to various TLR agonists and their activation can be enhanced by using combinations of TLR agonists. Pam3Cys/Poly I:C induced optimal cytokine production and increased cross-presentation. In addition, Flt3LDCs activated with this combination were found to confer tumour protection in vivo, albeit to a lesser degree than GMCSF/IL-4 DCs. In conclusion, Flt3L-cDCs can be used for DCbased immunotherapy, but may be less effective than GMCSF/IL-4 DCs. Further experiments are required to identify conditions whereby their effectiveness is maximised. 
$\underline{\text { References }}$ 
1. Ehrlich P. 1909. Ueber den jetzigen Stand der Karzinomforschung. Ned Tijdschr Geneeskd 5: 273-90

2. Dunn GP, Old LJ, Schreiber RD. 2004. The three Es of cancer immunoediting. Annu Rev Immunol 22: 329-60

3. LJ Old, EA Boyse. 1964. Immunology of Experimental Tumors. Annu Rev Med 15: $167-86$

4. G Klein. 1966. Tumour Antigens. Annu Rev Microbiol 20: 223-52

5. Dunn GP, Bruce AT, Ikeda H, Old LJ, RD. S. 2002. Cancer immunoediting: from immunosurveillance to tumor escape. Nat Immunol 3: 991-8

6. RD Schreiber, LJ Old, MJ Smyth. 2011. Cancer Immunoediting: Integrating Immunity's Roles in Cancer Suppression and Promotion. Science 331: 1565-70

7. Stutman O. 1974. Tumor development after 3-methylcholanthrene in immunologically deficient athymic-nude mice. Science 183: 534-36

8. Dunn GP, Koebel CM, Schreiber RD. 2006. Interferons, immunity and cancer immunoediting. Nat Rev Immunol 6: 836-48

9. Shankaran V, Ikeda H, Bruce AT, White JM, Swanson PE, Old LJ, Schreiber RD. 2001. IFNgamma and lymphocytes prevent primary tumour development and shape tumour immunogenicity. Nature 410: 1107-11

10. Smyth MJ, Crowe NY, Godfrey DI. 2001. NK cells and NKT cells collaborate in host protection from methylcholanthrene-induced fibrosarcoma. Int Immunol 4: 459-63

11. Whiteside TL. 2008. The tumour microenvironment and its role in promoting tumour growth Oncogene 27: 5905-12

12. Naito K SY, Shiiba K, Ohuchi A, Saigenji K, Nagura H, and Ohtani H. 1998. CD8+ T Cells Infiltrated within Cancer Cell Nests as a Prognostic Factor in Human Colorectal Cancer. Cancer Res 58: 3491-4

13. Sato E, Olson SH, Ahn J, Bundy B , Nishikawa H, Qian F, Jungbluth AA, Frosina D, Gnjatic S, Ambrosone C, Kepner J , Odunsi T, Ritter G, Lele S, Chen Y-T, Ohtani H, Old LJ, Odunsi K. 2005. Intraepithelial CD8+ tumor-infiltrating lymphocytes and a high $\mathrm{CD} 8+/$ regulatory $\mathrm{T}$ cell ratio are associated with favorable prognosis in ovarian cancer. Proc Natl Acad Sci USA 102: 18531-43

14. Kinlen LJ, Sheil AG, Peto J, Doll R. 1979. Collaborative United KingdomAustralasian study of cancer in patients treated with immunosuppressive drugs. $\mathrm{Br}$ Med J 2: 1461-6

15. Buell JF, Gross TG, Woodle ES. 2005. Malignancy after transplantation. Transplantation 80: S254-S64

16. Vesely MD, Kershaw MH, Schreiber RD, Smyth MJ. 2011. Natural innate and adaptive immunity to cancer. Annu Rev Immunol 29: 235-71

17. K Ryungsa, M Emi, K Tanabe. 2007. Cancer immunoediting from immune surveillance to immune escape. Immunol Rev 121: 1-14 
18. Koebel CM, Vermi W, Swann JB, Zerafa N, Rodig SJ, Old LJ, Smyth MJ, Schreiber RD. 2007. Adaptive immunity maintains occult cancer in an equilibrium state. Nature 450: 03-907

19. Tabi Z, Man S. 2006. Challenges for cancer vaccine development. Adv Drug Deliv Rev 58: $902-15$

20. P van der Bruggen, C Traversari, P Chomez, C Lurquin, E De Plaen, B Van den Eynde, A Knuth, T Boon. 1991. A gene encoding an antigen recognized by cytolytic T lymphocytes on a human melanoma. Science 254 1643-7

21. Traversari C, van der Bruggen P, Luescher IF, Lurquin C, Chomez P, Van Pel A, De Plaen E, Amar-Costesec A, Boon T. 1992. A nonapeptide encoded by human gene MAGE-1 is recognized on HLA-A1 by cytolytic T lymphocytes directed against tumor antigen MZ2-. J Exp Med 176: 1453-7

22. Antonio J, Spagnolib GC, Schultz-Thaterb E, Sarcevic B. 2003. Cancer/testis tumour-associated antigens: immunohistochemical detection with monoclonal antibodies. Lancet Oncol 4: 104-9

23. Wang R-F, Appella E, Kawakami Y, Kang X, Rosenberg SA. 1996. Identification of TRP-2 as a Human Tumor Antigen Recognized by Cytotoxic T Lymphocytes. $J$ Exp Med 184: 2207-16

24. Kawakami Y, Wang X, Shofuda T, Sumimoto H, Tupesis J, Fitzgerald E, Rosenberg S. 2001. Isolation of a new melanoma antigen, MART-2, containing a mutated epitope recognized by autologous tumor-infiltrating T lymphocytes. $J$ Immunol 166: 2871-7

25. Yotnda P, Firat H, Garcia-Pons F, Garcia Z, Gourru G, Vernant JP, Lemonnier FA, Leblond V, Langlade-Demoyen P. 1998. Cytotoxic T cell response against the chimeric p210 BCR-ABL protein in patients with chronic myelogenous leukemia. $J$ Clin Invest 101: 2290-6

26. Adam JK, Odhav B, Bhoola KD. 2003. Immune responses in cancer. Pharmacol Ther 99: 113-32

27. Toi M, Taniguchi T, Yamamoto Y, Kurishaki T, Suziki H, Tominaga T. 1996. Clinical sigificance of the determination of angiogenic factors. Eur J Cancer 32A: 2513-29

28. Ohm JE, Carbone DP. 2001. VEGF as a mediator of tumor-associated immunodeficiency. Immunol Res 23: 263-72

29. McMahon G. 2000. VEGF receptor signaling in tumor angiogenesis. Oncologist 5: 3-10

30. Oyama T, Ran S, Ishida T, Nadaf S, Kerr L, Carbone DP, Gabrilovich DI. 1998. Vascular endothelial growth factor affects dendritic cell maturation through the inhibition of nuclear factor-kappa B activation in hemopoietic progenitor cells. $J$ Immunol 160: 1224-32

31. Wrzesinski SH, Wan YY, Flavell RA. 2007. Transforming growth factor-beta and the immune response: implications for anticancer therapy. Clin Cancer Res 13: $5262-70$ 
32. Gold LI. 1999. The role for transforming growth factor-beta (TGF-beta) in human cancer. Crit Rev Oncog 10: 303-60

33. Aruga A, Aruga E, Tanigawa K, Bishop DK, Sondak VK, Chang AE. 1997. Type 1 versus type 2 cytokine release by Vbeta $\mathrm{T}$ cell subpopulations determines in vivo antitumor reactivity: IL-10 mediates a suppressive role. J Immunol 159: 664-73

34. Terabe M, Berzofsky JA. 2004. Immunoregulatory T cells in tumor immunity. Curr Opin Immunol 16: 157-62

35. Lewis CE, Pollard JW. 2006. Distinct Role of Macrophages in Different Tumor Microenvironments. Cancer Res 66: 605-12

36. Elgert KD, Alleva DG, Mullins DW. 1998. Tumor-induced immune dysfunction: the macrophage connection. J Leukoc Biol 64: 275-90

37. Leek RD, Lewis CE, Whitehouse R, Greenall M, Clarke J, Harris AL. 1996. Association of Macrophage Infiltration with Angiogenesis and Prognosis in Invasive Breast Carcinoma. Cancer Res 56: 42625-9

38. Hanada T, Nakagawa M, Emoto A, Nomura T, Nasu N, Nomura Y. 2000. Prognostic value of tumor-associated macrophage count in human bladder cancer. Int J Urol 7: 263-9

39. Schuster M, Nechansky A, Loibner H, Kircheis R. 2006. Cancer immunotherapy. Biotechnol J 1: 138-47

40. Eisenbach L, Bar-Haim E, El-Shami K. 2000. Antitumor vaccination using peptide based vaccines. Immunol Lett 74: 27-34

41. Sridhar T, Symonds RP. 2006. Principles of chemotherapy and radiotherapy. Obstet Gyneco Repro Med 16: 100-6

42. Nencioni A, Grünebach F, Schmidt SM, Müller MR, Boy D, Patrone F, Ballestrero A, Brossart P. 2008. The use of dendritic cells in cancer immunotherapy. Crit Rev Oncol Hematol 65: 191-9

43. Rosenberg SA, Lotze MT, Muul LM, Leitman S, Chang AE, Ettinghausen SE, Matory YL, Skibber JM, Shiloni E, Vetto JT, Seipp CA, Simpson C, Reicher CM. 1985. Observations on the systemic administration of autologous lymphokineactivated killer cells and recombinant interleukin-2 to patients with metastatic cancer. N Engl J Med 313: 1485-92

44. Rosenberg SA. 2001. Progress in human tumour immunology and immunotherapy. Nature 411: 380-4

45. Blattman JN, Greenberg PD. 2004. Cancer immunotherapy: a treatment for the masses. Science 305: 200-5

46. Steinman RM, Mellman I. 2004. Immunotherapy: bewitched, bothered, and bewildered no more. Science 305: 197-200

47. Bocchia M, Bronte V, Colombo MP, De Vincentiis A, Di Nicola M, Forni G, Lanata L, Lemoli RM, Massaia M, Rondelli D, Zanon P, Tura S. 2000. Antitumor vaccination: where we stand. Haematologica 85: 1172-206

48. Banchereau J, Palucka AK. 2005. Dendritic cells as therapeutic vaccines against cancer Nat Rev Immunol 5: 296-306 
49. Steinman RM, Dhodapkar M. 2001. Active immunization against cancer with dendritic cells: the near future. Int J Cancer 94: 459-73

50. Lesterhuis WJ, Aarntzen EH, De Vries IJM, Shuurhuis DH, Figdor CG, Adema GJ, Punt CJA. 2008. Dendritic cell vaccines in melanoma: From promise to proof? Crit Rev Oncol Hematol 66: 118-34

51. Dallal RM, Lotze MT. 2000. The dendritic cell and human cancer vaccines. Curr Opin Immunol 12: 583-8

52. Timmerman JM, Levy R. 1999. Dendritic cell vaccines for cancer immunotherapy. Annu Rev Med 50: 507-29

53. Mayordomo JI, Zorina T, Storkus WJ, Zitvogel L, Celluzzi C, Falo LD, Melief CJ, Ildstad ST, Kast WM, Deleo AB, Lotze MT. 1995. Bone marrow-derived dendritic cells pulsed with synthetic tumour peptides elicit protective and therapeutic antitumour immunity. Nat Med 1: 1297 - 302

54. Celluzzi CM, Mayordomo JI, Storkus WJ, Lotze MT, Falo LD Jr. 1996. Peptidepulsed dendritic cells induce antigen-specific CTL-mediated protective tumor immunity. J Exp Med 183: 283-7

55. Porgador A, Snyder D, Gilboa E. 1996. Induction of antitumor immunity using bone marrow-generated dendritic cells. J Immunol 156: 2918-26

56. Nair SK, Boczkowski D, Snyder D, Gilboa E. 1997. Antigen-presenting cells pulsed with unfractionated tumor-derived peptides are potent tumor vaccines. Eur $J$ Immunol 27: 589-97

57. Boullart AC, Aarntzen EH, Verdijk P, Jacobs JF, Schuurhuis DH, Benitez-Ribas D, Schreibelt G, van de Rakt MW, Scharenborg NM, de Boer A, Kramer M, Figdor CG, Punt CJ, Adema GJ, de Vries IJ. 2008. Maturation of monocyte-derived dendritic cells with Toll-like receptor 3 and 7/8 ligands combined with prostaglandin E2 results in high interleukin-12 production and cell migration. Cancer Immunol Immunother 57

58. Tacken PJ, de Vries IJ, Torensma R, Figdor CG. 2007. Dendritic-cell immunotherapy: from ex vivo loading to in vivo targeting. Nat Rev Immunol 7: 790-802

59. O'Neill DW, Adams S, Bhardwaj N. 2004. Manipulating dendritic cell biology for the active immunotherapy of cancer. Blood 104: 2235-46

60. Figdor CG, de Vries IJ, Lesterhuis WJ, Melief CJ. 2004. Dendritic cell immunotherapy: mapping the way. Nat Med 10: 475-80

61. Jefford E, Marakovsky E, Cebon J, Davis ID. 2001. The use of dendritic cells in cancer therapy. Lancet Oncol 2: 343-53

62. Ronchese F, Hausmann B. 1993. B lymphocytes in vivo fail to prime naive T cells but can stimulate antigen-experienced T lymphocytes. $J$ Exp Med 177: 679-90

63. Delamarre L, Pack M, Chang H, Mellman I, Trombetta ES. 2005. Differential Lysosomal Proteolysis in Antigen-Presenting Cells Determines Antigen Fate Science 307: 1630-4

64. Banchereau J, Steinman RM. 1998. Dendritic cells and the control of immunity. Nature Rev 392: 245-51 
65. Wu L, Liu Y-J. 2007. Development of Dendritic-Cell Lineages. Immunity Rev 26: $741-7$

66. Banchereau J, Briere F, Caux C, Davoust J, Lebecque S, Liu YJ, Pulendran B, Palucka K. 2000. Immunobiology of dendritic cells. Annu Rev Immunol 18: 767811

67. Shurin MR. 1996. Dendritic cells presenting tumour antigens. Cancer Immunol Immunother 43: 158-64

68. Shortman K, Naik SH. 2007. Steady-state and inflammatory dendritic-cell development. Nat Rev Immunol 7: 19-30

69. Kondo M, Weissman IL, Akashi K. 1997. Identification of clonogenic common lymphoid progenitors in mouse bone marrow. Cell 91: 661-72

70. Akashi K, Traver D, Miyamoto T, Weissman IL. 2000. A clonogenic common myeloid progenitor that gives rise to all myeloid lineages. Nature 404: 193-7

71. Manz MG, Traver D, Miyamoto T, Weissman IL, K. A. 2001. Dendritic cell potentials of early lymphoid and myeloid progenitors. Blood 97: 3333-41

72. Wu L, D'Amico A, Hochrein H, O'Keeffe M, Shortman K, Lucas K. 2001. Development of thymic and splenic dendritic cell populations from different hemopoietic precursors. Blood. 98: 3376-82

73. Graf T. 2002. Differentiation plasticity of hematopoietic cells. Blood 99: 3089-101

74. Karsunky H, Merad M, Cozzio A, Weissman IL, Manz MG. 2003. Flt3 ligand regulates dendritic cell development from Flt3+ lymphoid and myeloid-committed progenitors to Flt3+ dendritic cells in vivo. J Exp Med 198: 305-13

75. Gilliland DG, Griffin JD. 2002. The roles of FLT3 in hematopoiesis and leukemia. Blood 100: 1532-42

76. D'Amico A, Wu L. 2003. The early progenitors of mouse dendritic cells and plasmacytoid predendritic cells are within the bone marrow hemopoietic precursors expressing Flt3. J Exp Med 198: 293-303

77. Wodnar-Filipowicz A. 2003. Flt3 ligand: role in control of hematopoietic and immune functions of the bone marrow. News Physiol Sci 18: 247-51

78. Naik SH. 2008. Demystifying the development of dendritic cell subtypes, a little. Immunol Cell Biol 85: 439-52

79. Cheers C, Haigh AM, Kelso A, Metcalf D, Stanley ER, Young AM. 1988. Production of colony-stimulating factors (CSFs) during infection: separate determinations of macrophage-, granulocyte-, granulocyte-macrophage-, and multiCSFs. Infect Immun 56: 247-51

80. Serbina NV, Salazar-Mather TP, Biron CA, Kuziel WA, Pamer EG. 2003. TNF/iNOS-producing dendritic cells mediate innate immune defense against bacterial infection. Immunity 19: 59-70

81. Gordon S, Taylor PR. 2005. Monocyte and macrophage heterogeneity. Nat Rev Immunol 5: 953-64

82. Merad M, Manz MG. 2009. Dendritic cell homeostasis. Blood 113: 3418-27 
83. Conti L, Gessani S. 2008. GM-CSF in the generation of dendritic cells from human blood monocyte precursors: Recent advances. Immunobiology 213: 859-70

84. Naik SH. 2008. Demystifying the development of dendritic cell subtypes, a little. In Immunology and Cell Biology, pp. 439-52

85. Guthridge MA, Stomski FC, Thomas D, Woodcock JM, Bagley CJ, Berndt MC, Lopez AF. 1998. Mechanism of activation of the GM-CSF, IL-3, and IL-5 family of receptors. Stem Cells 16: 301-13

86. Maraskovsky E, Brasel K, Teepe M, Roux ER, Lyman SD, Shortman K, McKenna HJ. 1996. Dramatic increase in the numbers of functionally mature dendritic cells in Flt3 ligand-treated mice: multiple dendritic cell subpopulations identified. $J$ Exp Med 184: 1953-62

87. Pulendran B, Smith JL, Caspary G, Brasel K, Pettit D, Maraskovsky E, Maliszewski CR. 1999. Distinct dendritic cell subsets differentially regulate the class of immune response in vivo. Pro Natl Acad Sci USA 96: 1036-41

88. Shortman K, Liu YJ. 2002. Mouse and human dendritic cell subtypes. Nat Rev Immunol 2: 151-61

89. Colonna M, Trinchieri G, Liu J-Y. 2003. Plasmacytoid dendritic cells in immunity. Nat Immunol 5: 1219 - 26

90. Iwasaki A, Medzhitov R. 2010. Regulation of adaptive immunity by the innate immune system. Science 327: 291-5

91. McKenna K, Beignon AS, Bhardwaj N. 2005. Plasmacytoid dendritic cells: linking innate and adaptive immunity. J Virol 79: 17-27

92. Barchet W, Cella M, Colonna M. 2005. Plasmacytoid dendritic cells--virus experts of innate immunity. Semin Immunol 17: 253-61

93. Ito T, Wang YH, Liu YJ. 2005. Plasmacytoid dendritic cell precursors/type I interferon-producing cells sense viral infection by Toll-like receptor (TLR) 7 and TLR9. Springer Semin Immunopathol 26: 221-9

94. Merad M, Ginhoux F, Collin M. 2008. Origin, homeostasis and function of Langerhans cells and other langerin-expressing dendritic cells. Nat Rev Immunol 8: 935-47

95. Jiang W, Swiggard WJ, Heufler C, Peng M, Mirza A, Steinman RM, Nussenzweig MC. 1995. The receptor DEC-205 expressed by dendritic cells and thymic epithelial cells is involved in antigen processing. Nature 375: 151-5

96. Ginhoux F, Collin MP, Bogunovic M, Abel M, Leboeuf M, Helft J, Ochando J, Kissenpfennig A, Malissen B, Grisotto M, Snoeck H, Randolph G, Merad M. 2007. Blood-derived dermal langerin+ dendritic cells survey the skin in the steady state. $J$ Exp Med 204: 3133-46

97. Bursch LS, Wang L, Igyarto B, Kissenpfennig A, Malissen B, Kaplan DH, Hogquist KA. 2007. Identification of a novel population of Langerin+ dendritic cells. J Exp Med 204: 3147-56

98. Bedoui S, Whitney PG, Waithman J, Eidsmo L, Wakim L, Caminschi I, Allan RS, Wojtasiak M, Shortman K, Carbone FR, Brooks AG, Heath WR. 2009. Cross- 
presentation of viral and self antigens by skin-derived CD103+ dendritic cells. Nat Immunol 10: 488-95

99. Vremec D, Pooley J, Hochrein H, Wu L, Shortman K. 2000. CD4 and CD8 expression by dendritic cell subtypes in mouse thymus and spleen. $J$ Immunol 164: 2978-86

100. De Smedt T, Pajak B, Muraille E, Lespagnard L, Heinen E, De Baetselier P, Urbain J, Leo O, Moser M. 1996. Regulation of dendritic cell numbers and maturation by lipopolysaccharide in vivo. $J$ Exp Med 184: 1413-24

101. Pooley JL, Heath WR, Shortman K. 2001. Cutting edge: intravenous soluble antigen is presented to CD4 T cells by CD8- dendritic cells, but cross-presented to CD8 T cells by CD8+ dendritic cells. J Immunol 166: 5327-30

102. Belz GT, Shortman K, Bevan MJ, Heath WR. 2005. CD8alpha+ dendritic cells selectively present $\mathrm{MHC}$ class I-restricted noncytolytic viral and intracellular bacterial antigens in vivo. J Immunol 175: 196-200

103. Belz GT, Smith CM, Eichner D, Shortman K, Karupiah G, Carbone FR, Heath WR. 2004. Cutting edge: conventional CD8 alpha+ dendritic cells are generally involved in priming CTL immunity to viruse. J Immunol 172: 1996-2000.

104. Schnorrer P, Behrens G MN, Wilson NS, Pooley JL, Smith CM, El-Sukkari D, Davey G, Kupresanin F, Li M, Maraskovsky E, Belz GT, Carbone FR, Shortman K, Heath WR, Villadangos JA. 2006. The dominant role of CD8+ dendritic cells in cross-presentation is not dictated by antigen capture. Pro Natl Acad Sci USA 103: 10729-34

105. Savina A, Peres A, Cebrian I, Carmo N, Moita C, Hacohen N, Moita LF, Amigorena S. 2009. The small GTPase Rac2 controls phagosomal alkalinization and antigen crosspresentation selectively in CD8(+) dendritic cell. Immunity 30: 544-55

106. Dudziak D, Kamphorst AO, Heidkamp GF, Buchholz VR, Trumpfheller C, Yamazaki S, Cheong C, Liu K, Lee HW, Park CG, Steinman RM, Nussenzweig MC. 2007. Differential antigen processing by dendritic cell subsets in vivo. Science 315: $107-11$

107. Edwards AD, Diebold SS, Slack EMC, Tomizawa H, Hemmi H, Kaisho T, Akira S, Sousa CR. 2003. Toll-like receptor expression in murine DC subsets: lack of TLR7 expression by $\mathrm{CD} 8 \mathrm{a}+\mathrm{DC}$ correlates with unresponsiveness to imidazoquinolines. Eur J Immunol 33: 827-33

108. Schulz O, Diebold SS, Chen M, N aslund TI, Nolte MA, Alexopoulou L, Azuma YT, Flavell RA, Liljestr om P, CR. eS. 2005. Toll-like receptor 3 promotes crosspriming to virus-infected cells. Nature 433: 887-92

109. Doxsee CL, Riter TR, Reiter MJ, Gibson SJ, Vasilakos JP, Kedl RM. 2003. The immune response modifier and Toll-like receptor 7 agonist S-27609 selectively induces IL-12 and TNF-alpha production in CD11c+CD11b+CD8- dendritic cells. J Immunol 171: 1156-63 
110. Lindquist RL, Shakhar G, Dudziak D, Wardemann H, Eisenreich T, Dustin ML, Nussenzweig MC. 2004. Visualizing dendritic cell networks in vivo. Nat Immunol 5: $1243-50$

111. Inaba K, Witmer-Pack M, Inaba M, Hathcock KS, Sakuta H, Azuma M, Yagita H, Okumura K, Linsley PS, Ikehara S, Muramatsu S, Hodes RJ, Steinman RM. 1994. The tissue distribution of the B7-2 costimulator in mice: abundant expression on dendritic cells in situ and during maturation in vitro. $J$ Exp Med 180: 1849-60

112. Sallusto F, Cela M, Danieli C, Lanzavecchia A. 1995. Dendritic cell use macropinocytosis and the mannose receptor to concentrate macromolecules in the major histocompatibility complex class II compartment: downregulation by ctyokines and bacterial products. $J$ Exp Med 182: 389-400

113. Fanger NA, Wardwell K, Shen L, Tedder TF, Guyre PM. 1996. Type I (CD64) and type II (CD32) Fc $\gamma$ receptor-mediated phagocytosis by human blood dendritic cells. J Immunol 157: 541-48

114. Platt N, da Silva RP, Gordon S. 1998. Recognizing death: the phagocytosis of apoptotic cells. Trends Cell Biol 8: 365-72

115. Inaba K, Inaba M, Naito M, Steinman RM. 1993. Dendritic cell progenitors phagocytose particulates, including bacillus Calmette-Guerin organisms and sensitize mice to mycobacterial antigens in vivo. J Exp Med 178: 479-88

116. Albert ML, Pearce SF, Francisco LM, Sauter B, Roy P, Silverstein RL, Bhardwaj N. 1998. Immature dendritic cells phagocytose apoptotic cells via avb5 and CD36, and cross-present antigens to cytotoxic lymphocytes. J Exp Med 188: 359-68

117. Albert ML, Sauter B, Bhardwaj N. 1998. Dendritic cells acquire antigen from apoptotic cells and induce class I-restricted CTLs. Nature 392: 86-9

118. Jiang A, Bloom O, Ono S, Cui W, Unternaehrer J, Jiang S, Whitney JA, Connolly J, Banchereau J, Mellman I. 2007. Disruption of E-cadherin-mediated adhesion induces a functionally distinct pathway of dendritic cell maturation. Immunity: 61024

119. Huang FP, Platt N, Wykes M, Major JR, Powell TJ, Jenkins CD, MacPherson GG. 2000. A discrete subpopulation of dendritic cells transports apoptotic intestinal epithelial cells to T cell areas of mesenteric lymph nodes. J Exp Med 191: 435-44

120. Reis e Sousa C. 2006. Dendritic cells in a mature age. Nat Rev Immunol 6: 476-83

121. Lutz MB, Schuler G. 2002 Immature, semi-mature and fully mature dendritic cells: which signals induce tolerance or immunity. Trends Immunol 23: 445-9

122. Adler AJ, Marsh DW, Yochum GS, Guzzo JL, Nigam A, Nelson WG, Pardoll DM. 1998. CD4+ T Cell Tolerance to Parenchymal Self-Antigens Requires Presentation by Bone Marrow-derived Antigen-presenting Cells. J Exp Med 187: 1555-64

123. Hawiger D, Inaba K, Dorsett Y, Guo M, Mahnke K, Rivera M, Ravetch JV, Steinman RM, Nussenzweig MC. 2001. Dendritic cells induce peripheral T cell unresponsiveness under steady state conditions in vivo. J Exp Med 194: 769-79

124. Liu K, Iyoda T, Saternus M, Kimura Y, Inaba K, RM. S. 2002. Immune tolerance after delivery of dying cells to dendritic cells in situ. $J$ Exp Med 196: 1091-7 
125. Hernandez J, Aung S, Redmond WL, Sherman LA. 2001. Phenotypic and functional analysis of CD8(+) T cells undergoing peripheral deletion in response to cross-presentation of self-antigen. $J$ Exp Med 194: 707-17

126. Steinman RM, Nussenzweig MC. 2002. Avoiding horror autotoxicus: the importance of dendritic cells in peripheral T cell tolerance. Proc Natl Acad Sci USA 99: $351-8$

127. Medzhitov R, Janeway C Jr. 2000. Innate immune recognition: mechanisms and pathways. Immunol Rev 173: 89-97

128. Janeway CA Jr, Medzhitov R. 2002. Innate immune recognition. Annu Rev Immunol 20: 197-216

129. West MA, Wallin RP, Matthews SP, Svensson HG, Zaru R, Ljunggren HG, Prescott AR, Watts C. 2004. Enhanced dendritic cell antigen capture via toll-like receptor-induced actin remodeling. Science 305: 1153-7

130. Reis e Sousa C, Stahl PD, Austyn JM. 1993. Phagocytosis of antigens by Langerhans cells in vitro. $J$ Exp Med 178: 509-19

131. Banchereau J, Steinman RM. 1998. Dendritic cells and the control of immunity. Nature Rev 392: 245-52

132. Winzler C, Rovere P, Rescigno M, Granucci F, Penna G, Adorini L, Zimmermann VS, Davoust J, Ricciardi-Castagnoli P. 1997. Maturation stages of mouse dendritic cells in growth factor-dependent long-term cultures. $J$ Exp Med 185: 317-28

133. Acuto O, Michel F. 2003. CD28-mediated co-stimulation: a quantitative support for TCR signalling. Nat Rev Immunol 3: 939-51

134. Sallusto F, Schaerli P, Loetscher P, Schaniel C, Lenig D, Mackay CR, Qin S, Lanzavecchia A. 1998. Rapid and coordinated switch in chemokine receptor expression during dendritic cell maturation. Eur J Immunol 28: 2760-9

135. Goriely S, Neurath MF, Goldman M. 2008. How microorganisms tip the balance between interleukin-12 family members. Nat Rev Immunol 8: 81-6

136. Langenkamp A, Messi M, Lanzavecchia A, Sallusto F. 2000. Kinetics of dendritic cell activation: impact on priming of $\mathrm{TH} 1, \mathrm{TH} 2$ and nonpolarized T cells. Nat Immunol 1: 311-6

137. Trinchieri G, Sher A. 2007. Cooperation of Toll-like receptor signals in innate immune defence. Nat Rev Immunol 7: 179-90

138. de Vries IJ, Lesterhuis WJ, Scharenborg NM, Engelen LP, Ruiter DJ, Gerritsen MJ, Croockewit S, Britten CM, Torensma R, Adema GJ, Figdor CG, Punt CJ. 2003. Maturation of dendritic cells is a prerequisite for inducing immune responses in advanced melanoma patients. Clin Cancer Res 9: 5091-100

139. Nestle FO, Farkas A, Conrad C. 2005. Dendritic-cell-based therapeutic vaccination against cancer. Curr Opin Immunol 17: 163-9

140. Lee AW, Truong T, Bickham K, Fonteneau JF, Larsson M, Da Silva I, Somersan S, Thomas EK, Bhardwaj N. 2002. A clinical grade cocktail of cytokines and PGE2 results in uniform maturation of human monocyte-derived dendritic cells: implications for immunotherapy. Vaccine 20: A8-A22 
141. Liu K-J. 2006. Dendritic Cell, Toll-Like Receptor, and The Immune System. Cancer Mol 2: 213-5

142. McGettrick AF, O'Neill LA. 2010. Localisation and trafficking of Toll-like receptors: an important mode of regulation. Curr Opin Immunol 22: 20-7

143. Takeda K, Shizuo A. 2005. Toll-like receptors in innate immunity. Int Immunol 17: $1-14$

144. Beutler B. 2000. Tlr4: central component of the sole mammalian LPS sensor. Curr Opin Immunol 12: 20-6

145. Smith KD, Andersen-Nissen E, Hayashi F, Strobe K, Bergman MA, Barrett SLR, Cookson BT, Aderem A. 2003. Toll-like receptor 5 recognizes a conserved site on flagellin required for protofilament formation and bacterial motility. Nat Immunol 4: $1247-53$

146. Kanzler H, FJ B, Hessel EM, Coffman RL. 2007. Therapeutic targeting of innate immunity with Toll-like receptor agonists and antagonists. Nat Med 12: 552-9

147. Hemmi H, Takeuchi O, Kawai T, Kaisho T, Sato S, Sanjo H, Matsumoto M, Hoshino K, Wagner H, Takeda K, Akira S. 2000. A Toll-like receptor recognizes bacterial DNA. Nature 408: 740-5

148. Kawai T, Akira S. 2011. Toll-like Receptors and Their Crosstalk with Other Innate Receptors in Infection and Immunity. Immunity 34: 637-50

149. Stern LJ, Wiley DC. 1994. Antigenic peptides biding by class I and class II histocompatability proteins. Structure 2: 245-51

150. Moore MW, Cabone FR, Bevan MJ. 1998. Introduction of soluble protein into the class I pathway of antigen processing and presentation. Cell 54: 777-85

151. Probst HC, van de Breok M. 2005. Priming of CTLs by lymphocytic choriomeningitis virus depends on dendritic cells. J Immunol 174: 3920-4

152. Uebel S, Tampe R. 1999. Specificity of the proteosome and the TAP transporter. Curr Opin Immunol 11: 203-8

153. Jensen PE. 2007. Recent advances in antigen processing and presentation. Nat Immunol

154. Sadasivan B, Lehner PJ, Ortmann B, Spies T, Cresswell P. 1996. Roles for calreticulin and a novel glycoprotein, tapasin, in the interaction of MHC class I molecules with TAP. Immunity 5: 103-14

155. Lehner PJ, Surman MJ, Cresswell P. 1998. Soluble tapasin restores MHC class I expression and function in the tapasin-negative cell line. Immunity 8: 221-31

156. Trombetta ES, Ebersold M, Garrett W, Pypaert M, Mellman I. 2003. Activation of lysosomal function during dendritic cell maturation. Science 299: 1400-3.

157. Khalil H, Brunet A, Saba I, Tera R, Sekaly P, Thibodeau J. 2003. The MHC class II beta chain cytoplasmic tail overcomes the invariant chain p35-encoded endoplasmic reticulum retention signal. Int Immunol 15: 1249-63

158. Lamb CA, Cresswell P. 1992. Assembly and transport properties of invariant chain trimers and HLA-DR-invariant chain complexes. J Immunol 148: 3478-82 
159. Watts C. 2004. The exogenous pathways for antigen presentation on major histocompatibility complex class II and CD1 molecules. Nat Immunol 5: 880-8

160. Cresswell P. 1990. Invariant chain structure and MHC class II function. Cell 84: 505-7

161. Riberdy JM, Newcomb JR, Surman MJ, Barbosa JA, Cresswell P. 1992. HLA-DR molecules from an antigen-processing mutant cell line are associated with invariant chain peptides. Nature 360: 474-7

162. Avva RR, Cresswell P. 1994. In vivo and in vitro formation and dissociation of HLA-DR complexes with invariant chain-derived peptides. Immunity 1: 763-74

163. Denzin LK, Cresswell P. 1995. HLA-DM induces CLIP dissociation from MHC class II alpha beta dimers and facilitates peptide loading. Cell 82: 155-65

164. Trombetta ES, Mellman I. 2005. Cell biology of antigen processing in vtiro and in vivo. Annu Rev Immunol 23: 245-51

165. Burgdorf S, Kautz A, Bohnert V, Knolle PA, Kurts C. 2007. Distinct pathways of antigen uptake and intracellular routing in CD4 and CD8 T cell activation. Science 316: $612-6$

166. Bevan MJ. 1976. Cross-priming for a secondary cytotoxic response to minor $\mathrm{H}$ antigens with $\mathrm{H}-2$ congenic cells which do not cross-react in the cytotoxic assay. $J$ Exp Med 143: 1283-8

167. Harding CV, Song R. 1994. Phagocytic processing of exogenous particulate antigens by macrophages for presentation by class I MHC molecules. $J$ Immunol 153: 4925-33

168. Norbury CC, Hewlettt LJ, Prescott AR, Shastri N, Watts C. 1995. Class I MHC presenation of exogenous soluble antigen via macropinocytosis in bone marrow macrophages. Immunity: 783-91

169. Ke Y, Kapp JA. 1996. Exogenous antigens gain access to the major histocompatibility complex class I processing pathway in B cells by receptormediated uptake. J Exp Med 184: 1179-84

170. Jung S, Unutmaz D, Wong P, Sano G, De los Santos K, Sparwasser T, Wu S, Vuthoori S, Ko K, Zavala F, Pamer EG, Littman DR, Lang RA. 2002. In vivo depletion of CD11c $(+)$ dendritic cells abrogates priming of CD8(+) $\mathrm{T}$ cells by exogenous cell-associated antigens. Immunity 204: 1037-47

171. Lin ML, Zhan Y, Villadangos JA, Lew AM. 2008. The cell biology of crosspresentation and the role of dendritic cell subsets. Immunol Cell Biol 86: 353-62

172. Pfeifer JC, Wick MJ, RL R, Findlay K, Normark SJ, Harding CV. 1993. Phagocytic processing of bacterial antigens for class I MHC presentation to T cells. Nature 361: 359-62

173. Wick MJ, Pfeifer JD. 1996. Major histocompatibility complex class I presentation of ovalbumin peptide 257-264 from exogenous sources: protein context influences the degree of TAP-independent presentation. Eur J Immunol 26: 2790-9.

174. Ramirez MC, Sigal LJ. 2002. Macrophages and Dendritic Cells Use the Cytosolic Pathway to Rapidly Cross-Present Antigen from Live, Vaccinia-Infected Cells. $J$ Immunol 169: 6733-674 
175. Kovacsovics-Bankowski M, Rock KL. 1995. A Phagosome-to-Cytosol Pathway for Exogenous Antigens Presented on MHC Class I Molecules. Science 267: 243-6

176. Guermonprez P, Savaneu L, Kleijmeer M, Davoust J, Van Edert P, Amigorena S. 2003. ER-phagosome fusion defines an MHC class I cross-presentation compartment in dendritic cells. Nature 425: 397-402

177. Ackerman AL, Kyritsis C, Tampe R, Cresswell P. 2003. Early phagosomes in dendritic cells form a cellular compartment sufficient for cross-presentation of exogenous antigens. Proc Natl Acad Sci USA 100: 12889-94

178. Guermonprez P, Valladeau J, Zitvogel L, Théry C, Amigorena S. 2002. Antigen presentation and T cell stimulation by dendritic cells. Annu Rev Immunol 20: 62167

179. Lenschow DJ, Walunas TL, Bluestone JA. 1996. CD28/B7 system of T cell costimulation. Annu Rev Immunol 14: 14:233-58

180. Linsley PS, Ledbetter JA. 1993. The role of the CD28 receptor during T cell responses to antigen. Annu Rev Immunol 11: 191-212

181. Boonen GJ, van Dijk AM, Verdonck LF, van Lier RA, Rijksen G, Medema RH. 1999. CD28 induces cell cycle progression by IL-2-independent down-regulation of p27kip1 expression in human peripheral T lymphocytes. Eur J Immunol 29: 78998

182. Boise LH, Minn AJ, Noel PJ, June CH, Accavitti MA, Lindsten T, Thompson C. 2010. CD28 costimulation can promote $\mathrm{T}$ cell survival by enhancing the expression of Bcl-xL. J Immunol 185: 3788-99

183. Iezzi G, Karjalainen K, Lanzavecchia A. 1998. The duration of antigenic stimulation determines the fate of naive and effector T cells. Immunity 8: 89-95

184. van Kooten C, Banchereau J. 2000. CD40-CD40 ligand. J Leukoc Biol 67: 2-17

185. Koch F, Stanzl U, Jennewein P, Janke K, Heufler C, Kämpgen E, Romani N, Schuler G. 1996. High level IL-12 production by murine dendritic cells: upregulation via MHC class II and CD40 molecules and downregulation by IL-4 and IL-10. J Exp Med 184: 741-6

186. Cella M, Scheidegger D, Palmer-Lehmann K, Lane P, Lanzavecchia A, Alber G. 1996. Ligation of CD40 on dendritic cells triggers production of high levels of interleukin-12 and enhances $\mathrm{T}$ cell stimulatory capacity: T-T help via APC activation. $J$ Exp Med 184: 747-52

187. Testi R, Phillips JH, Lanier LL. 1998. T cell activation via Leu-23 (CD69). J Immunol 143: 1123-8

188. Szamel M, Appel A, Schwinzer R, Resch K. 1998. Different protein kinase C isoenzymes regulate IL-2 receptor expression or IL-2 synthesis in human lymphocytes stimulated via the TCR. J Immunol 160: 2207-14

189. Sallusto F, Mackay CR, Lanzavecchia A. 2000. The role of chemokine receptors in primary, effector, and memory immune responses. Annu Rev Immunol 18: 593-620

190. Bird JJ, Brown DR, Mullen AC, Moskowitz NH, Mahowald MA, Sider JR, Gajewski TF, Wang CR, Reiner SL. 1998. Helper T cell differentiation is controlled by the cell cycle. Immunity 9: 229-37 
191. Abbas AK, Murphy KM, Sher A. 1996. Functional diversity of helper T lymphocytes. Nature 383: 787-93

192. Zhu J, Guo L, Watson CJ, Hu-Li J, Paul WE. 2001. Stat6 is necessary and sufficient for IL-4's role in Th2 differentiation and cell expansion. $J$ Immunol 166: 7276-81

193. Diehl S, Rincón M. 2002. The two faces of IL-6 on Th1/Th2 differentiation. Mol Immunol. 39: 531-36

194. O'Garra A. 1998. Cytokines induce the development of functionally heterogeneous T helper cell subsets. Immunity 8: 275-83.

195. Jung S, Unutmaz D, Wong P, Sano G, De los Santos K, Sparwasser T, Wu S, Vuthoori S, Ko K, Zavala F, Pamer EG, Littman DR, RA L. 2002. In vivo depletion of $\mathrm{CD} 11 \mathrm{c}+$ dendritic cells abrogates priming of CD8 $+\mathrm{T}$ cells by exogenous cell-associated antigens. Immunity 17: 211-20

196. Barry M, Bleackley RC. 2002. Cytotoxic T lymphocytes: all roads lead to death. Nat Rev Immunol 2: 401-9

197. Liu CC, Walsh CM, Young JD. 1995. Perforin: structure and function. Immunol Today 16: 194-201

198. van Elsas A, Sutmuller RP, Hurwitz AA, Ziskin J, Villasenor J, Medema JP, Overwijk WW, Restifo NP, Melief CJ, Offringa R, Allison JP. 2001. Elucidating the autoimmune and antitumor effector mechanisms of a treatment based on cytotoxic T lymphocyte antigen-4 blockade in combination with a B16 melanoma vaccine: comparison of prophylaxis and therapy. J Exp Med 194: 481-89

199. Voskoboinik I, Smyth MJ, Trapani JA. 2006. Perforin-mediated target-cell death and immune homeostasis. Nat Rev Immunol 6: 940-52

200. Shi L, Mai S, Israels S, Browne K, Trapani JA, Greenberg AH. 1997. Granzyme B (GraB) autonomously crosses the cell membrane and perforin initiates apoptosis and GraB nuclear localization. J Exp Med 185: 855-66

201. Lavrik I, Golk A, Krammer PH. 2005. Death receptor signaling. J Cell Sci 118: $265-7$

202. Watanabe-Fukunaga R, Brannan CI, Itoh N, Yonehara S, Copeland NG, Jenkins NA, Nagata S. 1992. The cDNA structure, expression, and chromosomal assignment of the mouse Fas antigen. J Immunol 148: 1274-9

203. Waring P, Müllbacher A. 1999. Cell death induced by the Fas/Fas ligand pathway and its role in pathology. Immunol Cell Biol 77: 312-7

204. Chinnaiyan AM, O'Rourke K, Tewari M, Dixit VM. 1995. FADD, a novel death domain-containing protein, interacts with the death domain of Fas and initiates apoptosis. Cell 81: 505-12

205. Curtsinger JM, Lins DC, Mescher MF. 2003. Signal 3 determines tolerance versus full activation of naive CD8 T cells: dissociating proliferation and development of effector function. $J$ Exp Med 197: 1141-51

206. Wajant H, Pfizenmaier K, Scheurich P. 2003. Tumor necrosis factor signaling. Cell Death Differ 10: 45-65 
207. Beatty G, Paterson Y. 2001. IFN-gamma-dependent inhibition of tumor angiogenesis by tumor-infiltrating $\mathrm{CD} 4+\mathrm{T}$ cells requires tumor responsiveness to IFN-gamma. J Immunol 166: 2276-82

208. Samuel CE. 2001. Antiviral actions of interferons. Clin Microbiol Rev 14: 778-809

209. Kaech SM, Wherry EJ, Ahmed R. 2002. Effector and memory T-cell differentiation: implications for vaccine development. Nat Rev Immunol 2: 251-62

210. Villadangos JA, Schnorrer P. 2007. Intrinsic and cooperative antigen-presenting functions of dendritic-cell subsets in vivo. Nat Rev Immunol 7: 543-55.

211. Paczesny S, Ueno H, Fay J, Banchereau J, Palucka AK. 2003. Dendritic cells as vectors for immunotherapy of cancer. Semin Cancer Biol 13: 439-47

212. Mohamadzadeh M, Berard F, Essert G, Chalouni C, Pulendran B, Davoust J, Bridges G, Palucka AK, Banchereaua J. 2001. Interleukin 15 Skews Monocyte Differentiation into Dendritic Cells with Features of Langerhans Cells. J Exp Med 194: 1013-20

213. Paczesny S, Ueno H, Fay J, Banchereau J, Palucka AK. 2003. Dendritic cells as vectors for immunotherapy of cancer. Semin Cancer Biol 13: 439-47

214. Xu Y, Zhan Y, Lew AM, Naik SH, Kershaw MH. 2007. Differential development of murine dendritic cells by GM-CSF versus Flt3 ligand has implications for inflammation and trafficking. J Immunol 179: 7577-84

215. Schuler G, Schuler-Thurner B, Steinman RM. 2003. The use of dendritic cells in cancer immunotherapy. Curr Opin Immunol 15: 138-47

216. Thurner B, Haendle I, Röder C, Dieckmann D, Keikavoussi P, Jonuleit H, Bender A, Maczek C, Schreiner D, von den Driesch P, Bröcker EB, Steinman RM, Enk A, Kämpgen E, Schuler G. 1999. Vaccination with mage-3A1 peptide-pulsed mature, monocyte-derived dendritic cells expands specific cytotoxic T cells and induces regression of some metastases in advanced stage IV melanoma. J Exp Med 190: 1669-78

217. Schuler-Thurner B, Schultz ES, Berger TG, Weinlich G, Ebner S, Woerl P, Bender A, Feuerstein B, Fritsch PO, Romani N, Schuler G. 2002. Rapid induction of tumor-specific type $1 \mathrm{~T}$ helper cells in metastatic melanoma patients by vaccination with mature, cryopreserved, peptide-loaded monocyte-derived dendritic cells. $J$ Exp Med 195: 1279-88

218. Vremec D, Lieschke GJ, Dunn AR, Robb L, Metcalf D, Shortman K. 1997. The influence of granulocyte/macrophage colony-stimulating factor on dendritic cell levels in mouse lymphoid organs. Eur J Immunol 27: 40-4

219. Naik SH, Metcalf D, van Nieuwenhuijze A, Wicks I, Wu L, O'Keeffe M, Shortman K. 2006. Intrasplenic steady-state dendritic cell precursors that are distinct from monocytes. Nat Immunol 7: 663-71

220. Hannum C, Culpepper J, Campbell D, McClanahan T, Zurawski S, Bazan JF, Kastelein R, Hudak S, Wagner J, Mattson J, Luh J, Duda G, Martina N, Petersen D, Menon S, Shanafelt A, Meuench M, Kelner G, Namikawa R, Rennick D, Roncarolo M-G, Zlotnik A, Rosnet O, Dubreuil P, Birnbaum D, Lee F. 1994. Ligand for FLT3/FLK2 receptor tyrosine kinase regulates growth of haematopoietic stem cells and is encoded by variant RNAs. Nature 368: 643-8 
221. Lyman SD, James L, Vanden Bos T, de Vries P, Brasel K, Gliniak B, Hollingsworth LT, Picha KS, McKenna HJ, Splett RR, Fletcher F, Maraskovsky E, Farrah T, Foxworthe D, Williams DE, Beckmann M. 1993. Molecular cloning of a ligand for the flt3/flk-2 tyrosine kinase receptor: a proliferative factor for primitive hematopoietic cell. Cell 75: 1157-116

222. McKenna HJ, Stocking KL, Miller RE, Brasel K, De Smedt T, Maraskovsky E, Maliszewski CR, Lynch DH, Smith J, Pulendran B, Roux ER, Teepe M, Lyman SD, Peschon JJ. 2000. Mice lacking flt3 ligand have deficient hematopoiesis affecting hematopoietic progenitor cells, dendritic cells, and natural killer cells. Blood 95: 3489-97

223. Tussiwand R, Onai N, Mazzucchelli L, Manz MG. 2005. Inhibition of natural type I IFN-producing and dendritic cell development by a small molecule receptor tyrosine kinase inhibitor with Flt3 affinity. J Immunol 175: 3674-80

224. Hawley TS, Fong AZ, Griesser H, Lyman SD, Hawley RG. 1998. Leukemic predisposition of mice transplanted with gene-modified hematopoietic precursors expressing flt3 ligand. Blood 92: 2003-11

225. Naik S, Proietto, AI, Wilson, NS, Aleksandar, D, Schnorrer P, Fuchsberger M, Lahoud MH, O'Keeffe M, Qi-xiang S, Wei-feng C, Villadangos JA, Shortman K, Wu L. 2005. Cutting edge: generation of splenic CD8+ and CD8-dendritic cell equivalents in Fms-like tyrosine kinase 3 ligand bone marrow cultures. J Immunol 174: 6592-7

226. Melcher A, Todryk S, Bateman A, Chong H, Lemoine NR, Vile RG. 1999. Adoptive transfer of immature dendritic cells with autologous or allogeneic tumor cells generates systemic antitumor immunity. Cancer Res 59: 2802-5

227. Sathe P, Pooley J, Vremec D, Mintern J, Jin JO, Wu L, Kwak JY, Villadangos JA, K. S. 2011. The acquisition of antigen cross-presentation function by newly formed dendritic cells. J Immunol 186: 5184-92

228. Barnden MJ, Allison J, Heath WR, Carbone FR. 1998. Defective TCR expression in transgenic mice constructed using cDNA-based alpha- and beta-chain genes under the control of heterologous regulatory elements. Immunol Cell Biol 76: 34-40

229. Hogquist KA, Jameson SC, Heath WR, Howard JL, Bevan MJ, Carbone FR. 1994. T cell receptor antagonist peptides induce positive selection. Cell 76: 17-27

230. Hoshino K, Takeuchi O, Kawai T, Sanjo H, Ogawa T, Takeda Y, Takeda K, Akira S. 1999. Cutting edge: Toll-like receptor 4 (TLR4)- deficient mice are hyporesponsive to lipopolysaccharide: evidence for TLR4 as the Lps gene product. J Immunol 162: 3749-52

231. Brasel K, De Smedt T, Smith JL, Maliszewski CR. 2000. Generation of murine dendritic cells from flt3-ligand-supplemented bone marrow cultures. Blood 96: 3029-39

232. Garrigan K, Moroni-Rawson P, McMurray C, Hermans I, Abernethy N, Watson J, Ronchese F. 1996. Functional comparison of spleen dendritic cells and dendritic cells cultured in vitro from bone marrow precursors. Blood 88: 3508-12 
233. Brawand P, Fitzpatrick DR, Greenfield BW, Brasel K, Maliszewski CR, De Smedt T. 2002. Murine Plasmacytoid Pre-Dendritic Cells Generated from Flt3 LigandSupplemented Bone Marrow Cultures Are Immature APCs. J Immunol 169: 6711-9

234. Angelov GS, Tomkowiak M, Marcais A, Leverrier Y, Marvel J. 2005. Flt3Ligand-Generated Murine Plasmacytoid and conventional Dendritic cells Differ in their Capacity to Prime Naive CD8 T Cells and to Generated Memory Cell In Vivo. J Immunol 175: 189-95

235. Napolitani G, Rinaldi A, Bertoni F, Sallusto F, Lanzavecchia A. 2005. Selected Toll-like receptor agonist combinations synergistically trigger a $\mathrm{T}$ helper type 1polarizing program in dendritic cells. Nat Immunol 6: 769-76

236. Warger T, Osterloh P, Rechtsteiner G, Fassbender M, Heib V, Schmid B, Schmitt E, Schild H, Radsak MP. 2006. Synergistic activation of dendritic cells by combined Toll-like receptor ligation induces superior CTL responses in vivo. Blood 108: $544-50$

237. Akira S, Takeda K. 2004. Toll-like receptor signalling. Nat Rev Immunol 4: 499511

238. Liew FY, Xu D, Brint EK, O’Neill L A.J. 2005. Negative regulation of Toll-like receptor mediated immune responses. Nat. Rev. Immunol 5: 446-58

239. Kawai T, Akira S. 2010. The role of pattern-recognition receptors in innate immunity: update on Toll-like receptors. Nat Immunol 11: 373-84

240. Bagchi H, Herrup EA, Warren HS, Trigilio J, Shin H-S, Valentine C, Hellman J. 2007. MyD88-Dependent and MyD88-Independent Pathways in Synergy, Priming, and Tolerance between TLR Agonists. J Immunol 178: 1164-71

241. Krauss S, Brand MD, Buttgereit F. 2001. Signaling takes a breath--new quantitative perspectives on bioenergetics and signal transduction. Immunity 15: 497-502

242. Fox CJ, Hammerman PS, Thompson CB. 2005. Fuel feeds function: Energy metabolism and the T-cell response. Nat Rev 5: 844-52

243. Jones RG, Thompson CB. 2007. Revving the Engine: Signal Transduction Fuels T Cell Activation. Immunity 27: 173-8

244. Frauwirth KA, Thompson CB. 2004. Regulation of T lymphocyte metabolism. $J$ Immunol 172: 4661-5

245. Krawczyk CM, Holowka T, Sun J, Blagih J, Amiel E, DeBeradinis RJ, Cross JR, Jung E, Thompson CB, Jone RG, Pearce EJ. 2010. Toll-like receptor-induced changes in glycolytic metabolism regulate dendritic cell activation. Blood 115: 4742-9

246. Soumelis V, Liu Y-J. 2006. From plasmacytoid to dendritic cell: Morphological and functional switches during plasmacytoid pre-dendritic cell differentiation. Eur. J. Immunol 36: 2286-92

247. Asslin-Paturel C, Boonstra A, Dalod M, Durand I, Yessaad N, DezutterDambuyant C, Vicari A, O'Gara A, Biron C, Briere F, Trinchieri G. 2001. Mouse type I IFN-producing cells are immature APCs with plasmacytoid morphology. Nat Immunol 2: 1144-50 
248. Labeur MS, Roters B, Pers B, Mehling A, Luger TA, Schwarz T, Grabbe S. 1999. Generation of tumor immunity by bone marrow-derived dendritic cells correlates with dendritic cell maturation stage. J Immunol 162: 168-75

249. Pelicano H, Martin DS, Xu R-H, Huang P. 2006. Glycolysis inhibition for anticancer treatment. Oncogene 25: 4633-46

250. Zhong D, Xiong L, Liu T, Liu X, Liu X, Chen J, Sun S-Y, Khuri FR, Zong YP, Zhou Q, Zhou W. 2009. The Glycolytic Inhibitor 2-Deoxyglucose Activates Multiple Prosurvival Pathways through IGF1R. J Biol Chem 284: 23225-33

251. Jonuleit H G-TA, Tüting T, Thurner-Schuler B, Stuge TB, Paragnik L, Kandemir A, Lee PP, Schuler G, Knop J, Enk AH,. 2001. A comparison of two types of dendritic cell as adjuvants for the induction of melanoma-specific T-cell responses in humans following intranodal injection. Int J Cancer 93: 243-51

252. Schreibelt G, Tel J, Sliepen K H. E. W. J, Benitez-Ribas D, Figdor CG, Adema GJ, de Vries I. JM. 2010. Toll-like receptor expression and function in human dendritic cell subsets: implications for dendritic cell-based anti-cancer immunotherapy. Cancer Immunol Immunother 59: 1573-82

253. Luft T, Jeord M, Luetjens P, Toy T, Hochrein H, Masterman KA, Maliszewski CR, Shortman K, Cebon J, Maraskovsky E. 2002. Functionally distinct dendritic cell (DC) populations induced by physiologic stimuli: prostaglandin $\mathrm{E}(2)$ regulates the migratory capacity of specific DC subsets. Blood 100: 1362-72

254. Sallusto F, Lanzavecchia A. 1994. Efficient presentation of soluble antigen by cultured human dendritic cells is maintained by granulocyte/macrophage colonystimulating factor plus interleukin 4 and downregulated by tumor necrosis factor alpha. J Exp Med 179: 1109-18

255. Brawand P, Fitzpatrick DR, Greenfield BW, Brasel K, Maliszewski CR, Smedt TD. 2002. Murine Plasmacytoid Pre-Dendritic cells Generated from Flt3-LSupplemented Bone Marrow Culture Are Immature APCs. J Immunol 169: 6711-9

256. Lee YJ, Galoforo SS, Berns CM, Chen JC, Davis BH, Sim JE, Corry PM, Spitz DR. 1998. Glucose deprivation-induced cytotoxicity and alterations in mitogenactivated protein kinase activation are mediated by oxidative stress in multidrugresistant human breast carcinoma cells. J Biol Chem 273: 5294-9

257. Iwasaki A, Medzhitov R. 2004. Toll-like receptor control of the adaptive immune responses. Nat Rev Immunol 5: 987-95

258. Schietinger A, Philip M, Liu RB, Schreiber K, Schreiber H. 2010. Bystander killing of cancer requires the cooperation of CD4+ and CD8+ T cells during the effector phase. J Exp Med 207: 2469-77

259. Marzo AL, Kinnear BF, Lake RA, Frelinger JJ, Collins EJ, Robinson BW, Scott B. 2000. Tumor-Specific CD4+ T Cells Have a Major "Post-Licensing" Role in CTL Mediated Anti-Tumor Immunity. J Immunol 165: 6047-55

260. Toes RE, Ossendorp F, Offringa R, Melief CJ. 1999. CD4 T cells and their role in antitumor immune responses. J Exp Med 189: 753-6

261. Pardoll DM, Topalian SL. 1998. The role of CD4+ T cell responses in antitumor immunity. Curr Opin Immunol 5: 588-94 
262. Wang RF. 2001. The role of MHC classII-restriced tumour antigens and CD4+ T cells in antitumor immunity. Trends Immunol 22: 269-76.

263. Kennedy R, Celis E. 2008. Multiple roles for CD4+ T cells in anti-tumour immune responses. Immunol Rev 222: 129-44

264. Hung K, Hayashi R, Lafond-Walker A, Lowenstein C, Pardoll D, Levitsky H. 1998. The Central Role of CD4 T Cells in the Antitumor Immune Response. J Exp Med 188: 2357-68

265. Haabeth OA, Lorvik KB, Hammarström C, Donaldson IM, Haraldsen G, Bogen B, Corthay A. 2011. Inflammation driven by tumour-specific Th1 cells protects against B-cell cancer. Nat Commun 2: 1-8

266. Farber JM. 1990. A macrophage mRNA selectively induced by y-interferon encodes a member of the platelet factor 4 family of cytokines. Proc Natl Acad Sci USA 87: 5238-42

267. Sgadari C, Farber JM, Angiolillo AL, Liao F, Teruya-Feldstein J, Burd PR YL, Gupta G, Kanegane C, Tosato G. 1997. Mig, the monokine induced by interferongamma, promotes tumor necrosis in vivo. Blood 89: 2635-43

268. Hu HM, Winter H, Urba WJ, Fox BA. 2000. Divergent roles for CD4+ T cells in the priming and effector/memory phases of adoptive immunotherapy. J Immunol 165: 4246-53

269. Shedlock DJ, Shen H. 2003. Requirement for CD4 T Cell Help in Generating Functional CD8 T Cell Memory. Science 300: 337-9

270. Janssen EM, Lemmen EE, Wolfe T, Christen U, von Herrath MG, Schoenberger SP. 2003. CD4+ $\mathrm{T}$ cells are required for secondary expansion and memory in CD8+ T lymphocytes Nature 421: 852-6

271. Ma X, Chow JM, Gri G, Carra G, Gersoa F, SF W, Dzialo R, Trinchieri G. 1996. The interleukin $12 \mathrm{p} 40$ gene promoter is primed by interferon gamma in monocytic cells. J Exp Med 183: 147-57

272. Curtsinger JM, Johnson CM, Mescher MF. 2003. CD8 T cell clonal expansion and development of effector function require prolonged exposure to antigen, costimulation, and signal 3 cytokine. J Immunol 171: 5165-71

273. Calzascia T, Pellegrini M, Hall H, Sabbagh L, Ono N, Elford AR, Mak TW, Ohashi PS. 2007. TNF-alpha is critical for antitumor but not antiviral $\mathrm{T}$ cell immunity in mice. J Clin Invest 117: 3833-45

274. MartIn-Fontecha A, Sebastiani S, Höpken UE, Uguccioni M, Lipp M, Lanzavecchia A, Sallusto F. 2003. Regulation of dendritic cell migration to the draining lymph node: impact on T lymphocyte traffic and priming. J Exp Med 198: $615-21$

275. Pasare P, Medzhito R. 2003. Toll Pathway-Dependent Blockade of CD4+CD25+ T Cell-Mediated Suppression by Dendritic Cell. Science 299: 1033-6

276. De Smedt T, Van Mechelen M, De Becker G, Urbain J, Leo O, Moser M. 1997. Effect of interleukin-10 on dendritic cell maturation and function. Eur J Immunol 5: 1229-35 
277. Colomboa MP, Trinchieri G. 2002. Interleukin-12 in anti-tumor immunity and immunotherapy. Cytokine Growth Factor Rev 13: 155-68

278. Nylander S, Kalies I. 1999. Brefeldin A, but not monensin, completely blocks CD69 expression on mouse lymphocytes: efficacy of inhibitors of protein secretion in protocols for intracellular cytokine staining by flow cytometry. $J$ Immunol Methods 224: 69-76

279. Guermonprez P, Valladeau J, Zitvogel L, Amigorena S. 2002. Antigen presentation and T cell stimulation by dendritic cells. Annu Rev Immunol 20: 620-67

280. Théry C, Amigorena S. 2001. The cell biology of antigen presentation in dendritic cells. Curr Opin Immunol 1: 45-51

281. Colombo MP, Trinchieri G. 2002. Interleukin-12 in anti-tumor immunity and immunotherapy. Cytokine Growth Factor Rev 13: 155-68

282. Trinchieri G. 2003. Interleukin-12 and the regulation of innate resistance and adaptie immunity. Nature Rev 3: 133-46

283. Kato H, Takeuchi O, Mikamo-Satoh E, Hirai R, Kawai T, Matsushita K, Hiiragi A, Dermody TS, Fujita T, Akira S. 2008. Length-dependent recognition of doublestranded ribonucleic acids by retinoic acid-inducible gene-I and melanoma differentiation-associated gene 5. J Exp Med 205: 1601-10

284. Kawai T, Akira S. 2008. Toll-like receptor and RIG-I-like receptor signaling. Ann NY Acad Sci 1143: 1-20

285. Luber CA, Cox J, Lauterbach H, Fancke B, Selbach M, Tschopp J, Akira S, Wiegand M, Hochrein H, O'Keeff M, Mann M. 2010. Quantitative Proteomics Reveals Subset-Specific Viral Recognition in Dendritic Cells. Immunity 32: 279-89

286. McKenna K, Beignon A-S, Bhardwaj N. 2005. Plasmacytoid Dendritic Cells: Linking Innate and Adaptive Immunity. J Virol 79: 17-27

287. Lenschow DJ, Walunas TL, Bluestone JA. 1996. CD28/B7 system of T cell costimulation. Annu. Rev. Immunol 14: 233-58

288. Delamarre L, Holcombe H, Mellman I. 2003. Presentation of Exogenous Antigens on Major Histocompatibiltiy Complex (MHC) Class I and MHC Class II molecules Is Differentially regulated during Dendritic Cell Maturation. J Exp Med 198: 11122

289. Pierre P, Turley SJ, Gatti E, Hull M, Meltzer J, Mirza A, Inaba K, Steinman RM, Mellman I. 1997. Developmental regulation of MHC class II transport in mouse dendritic cells. Nature 388: 787-92

290. de Brito C, Tomkowiak M, Ghittoni R, Caux C, Leverrier Y, Marvel J. 2010. CpG promotes cross-presentation of dead cell-associated antigens by pre-CD $8 \alpha+$ dendritic cells. J Immunol 186: 1503-11

291. Jancic C, Savina A, Wasmeier C, Tolmachova T, El-Benna J, Dang PM, Pascolo S, Gougerot-Pocidalo MA, Raposo G, Seabra MC, S. A. 2007. Rab27a regulates phagosomal $\mathrm{pH}$ and NADPH oxidase recruitment to dendritic cell phagosomes. Nat Cell Biol 9: 367-78

292. Jonuleit H, Giesecke-Tuettenberg A, Tüting T, Thurner-Schuler B, Stuge TB, Paragnik L, Kandemir A, Lee PP, Schuler G, Knop J, Enk AH. 2001. A comparison 
of two types of dendritic cell as adjuvants for the induction of melanoma-specific T-cell responses in humans following intranodal injection. Int J Cancer 93: 243-51

293. Hirao M, Onai N, Hiroishi K, Watkins SC, Matsushima K, Robbins PD, Lotze MT, Tahara H. 2000. CC chemokine receptor-7 on dendritic cells is induced after interaction with apoptotic tumor cells: critical role in migration from the tumor site to draining lymph nodes. Cancer Res 60: 2209-17.

294. Stutte S, Quast T, Gerbitzki N, Savinko T, Novak N, Reifenberger J, Homey B, Kolanus W, Alenius H, Förster I. 2010. Selected natural and synthetic retinoids impair CCR7- and CXCR4-dependent cell migration in vitro and in vivo. Proc Natl Acad Sci U S A 107: 8736-41 
$\underline{\text { Appendices }}$ 

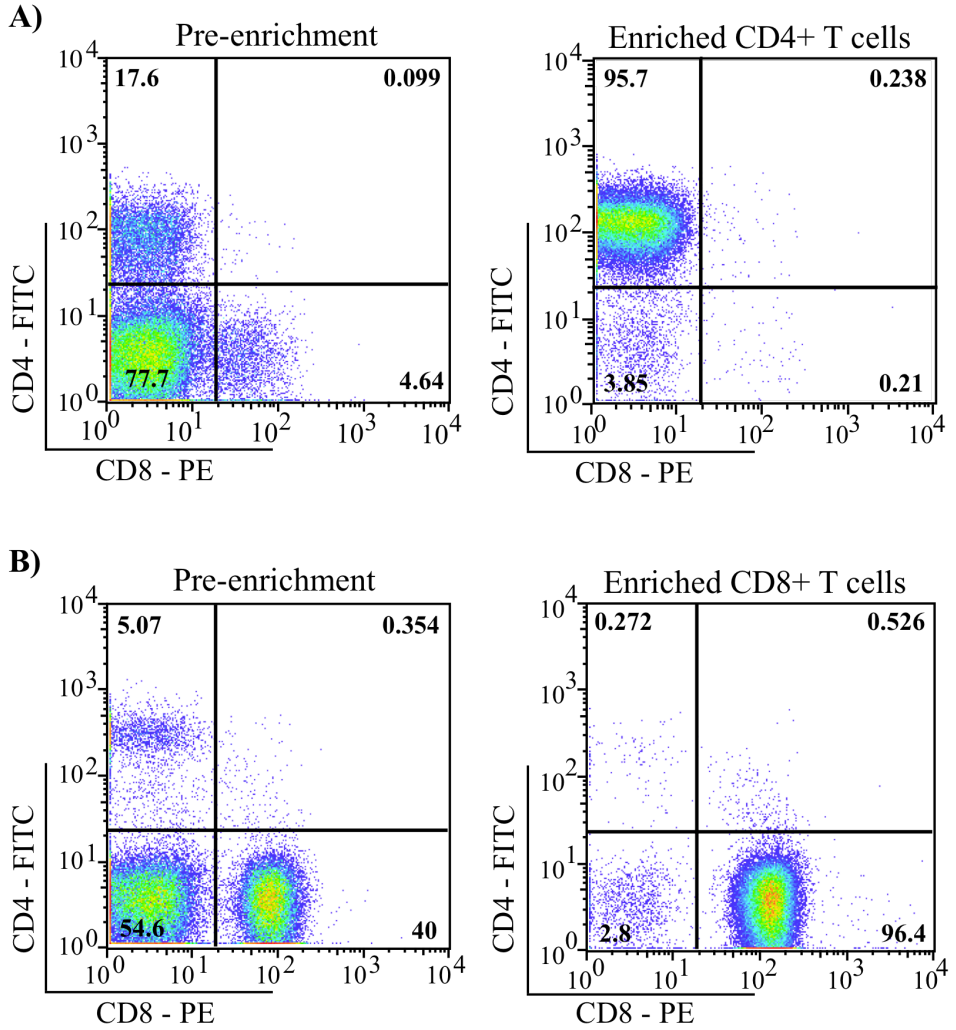

\section{Appendix 1: $\mathrm{CD4}^{+}$and $\mathrm{CD8}^{+} \mathrm{T}$ cells are enriched from $\mathrm{LN}$ and spleens of OT-II and}

OTI mice following positive selection. Lymphocytes and splenocytes were enriched for $\mathrm{CD}^{+} \mathrm{T}$ cells (A) and $\mathrm{CD}^{+} \mathrm{T}$ cells (B) by magnetic cell separation as described in Chapter 2 (2.4.3.2). Aliquots of pre-enriched and enriched samples were analysed for expression of CD4 and CD8 by flow cytometry. Numbers indicate the percentage of cells within respective gating regions. This result is representative of several experiments. 

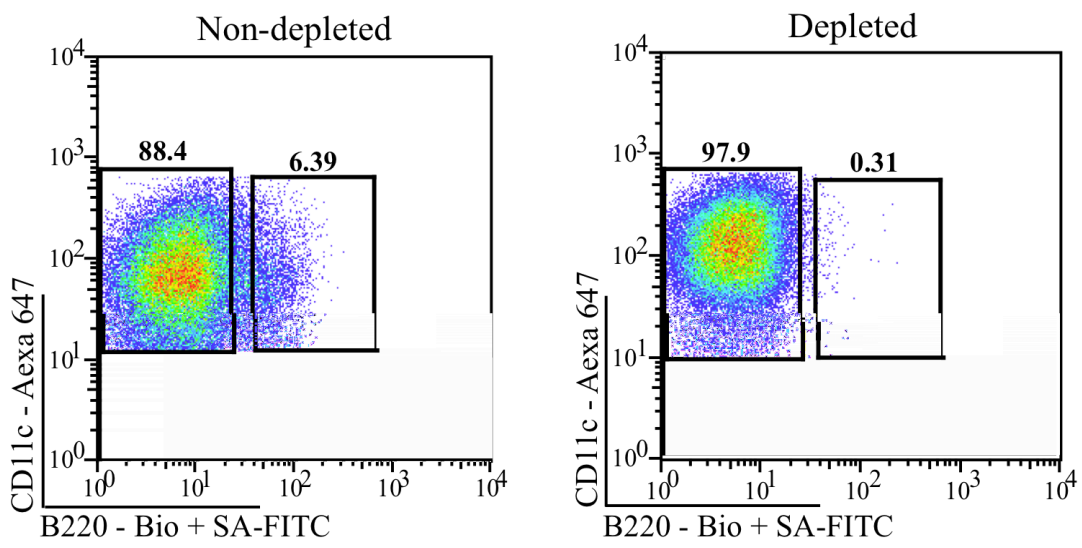

Appendix 2: Purity of cDCs recovered following magnetic cell separation (MACS). Flt3L-DC cultures were harvested and depleted of $\mathrm{B} 220^{+} \mathrm{pDCs}$ as described in Chapter 2 (2.4.3.3). Aliquot of non-depleted and depleted samples were analysed by flow cytometry to evaluate whether B220 $0^{+} \mathrm{pDCs}$ were depleted. Numbers indicate the percentage of cells within respective gating regions. This result is representative of several experiments. 
A) cDCs
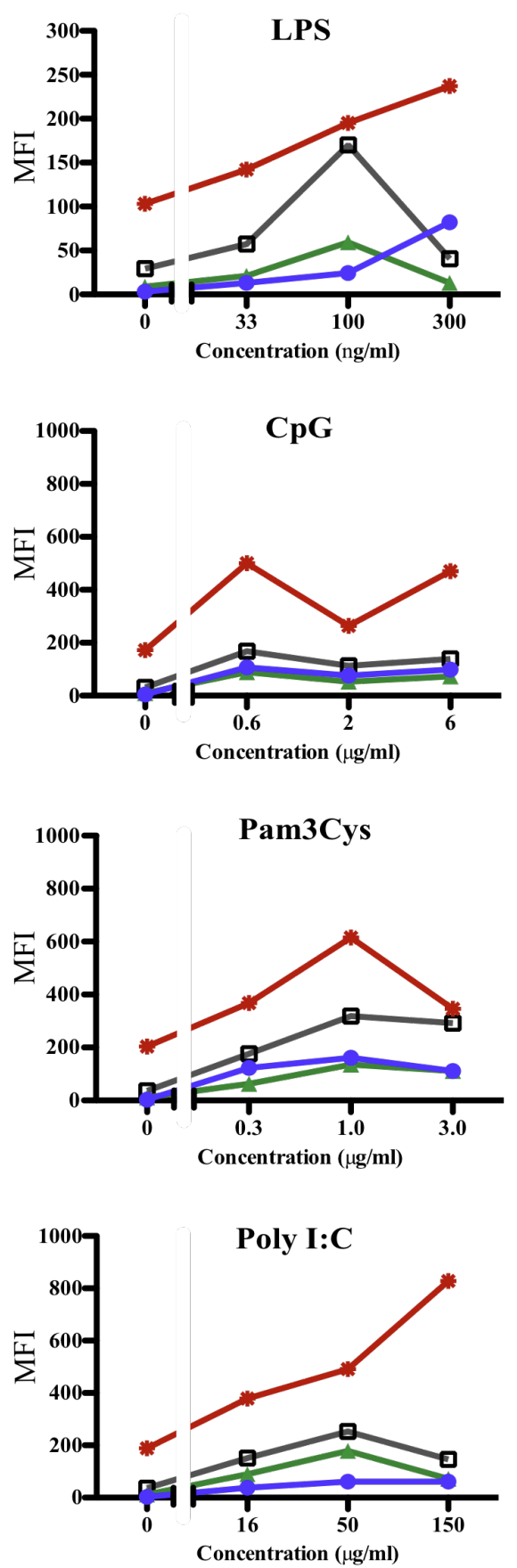

B) $\mathrm{pDCs}$
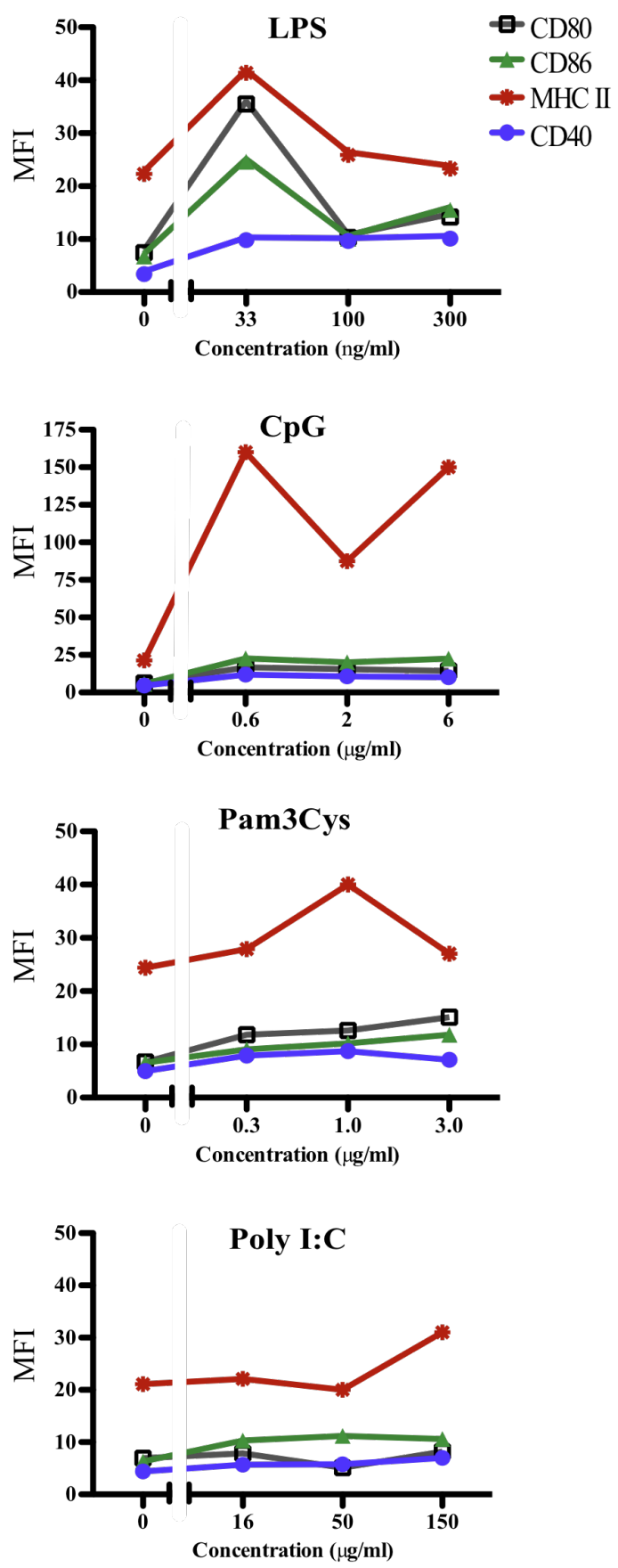

\section{Appendix 3: Titration of TLR agonists on Flt3L-DCs}

Flt3L-DCs were generated as described in Chapter 2 (2.4.2.2). On day 9 of cell culture, Flt3L-DCs were stimulated with TLR agonists at varying concentrations and incubated for 24 hours. Cells were harvested the following day and stained with varying combinations of mAbs for the markers CD11c, B220, CD80, CD86, CD40 and MHC II. Samples were analysed by flow cytometry. Results are from one experiment only. 
A)
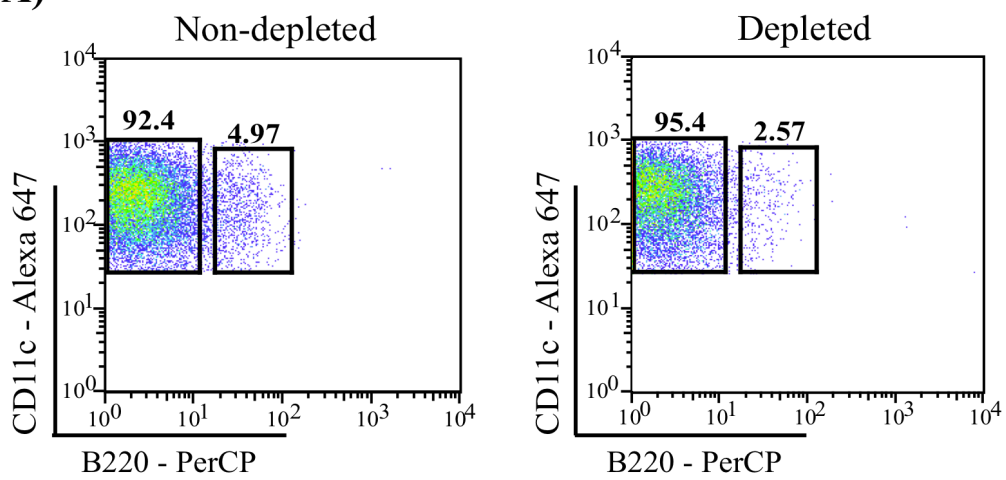

B)
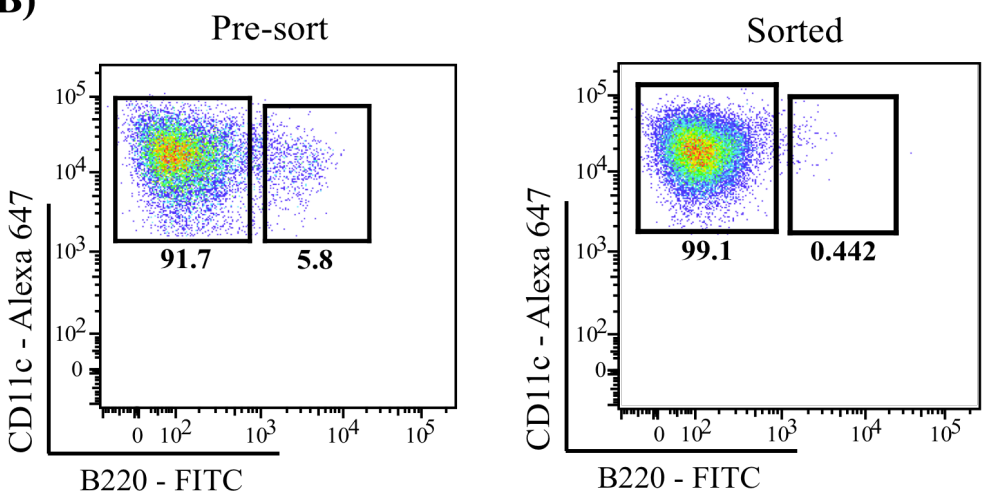

\section{Appendix 4: Purity of cDCs recovered following depletion with using BioMag®} beads or by Fluorescence Activated Cell Sorting (FACS). Flt3L-DC cultures were harvested and depleted of B220 $0^{+}$pDCs by magnetic bead separation using BioMag® beads (A) or by fluorescent activated cell sorting (B), as described in Chapter 2 (2.4.3.4 and 2.4.3.5 respectively). Non-depleted and depleted or sorted and pre-sorted samples were analysed by flow cytometry. Numbers indicate percentage of cells within respective gating regions. Results are from one experiment only. 
A)
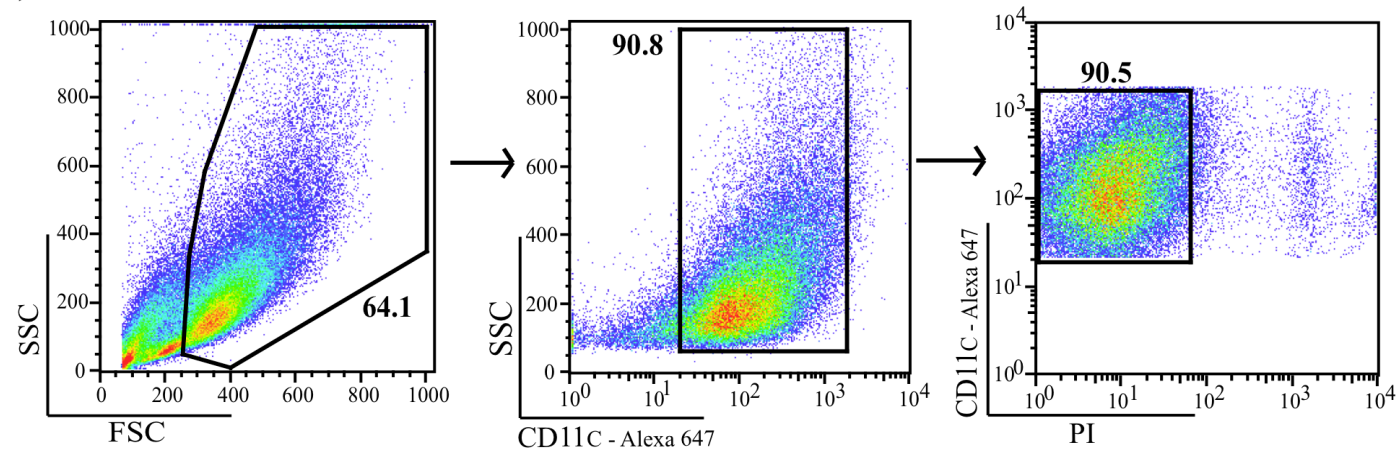

B)
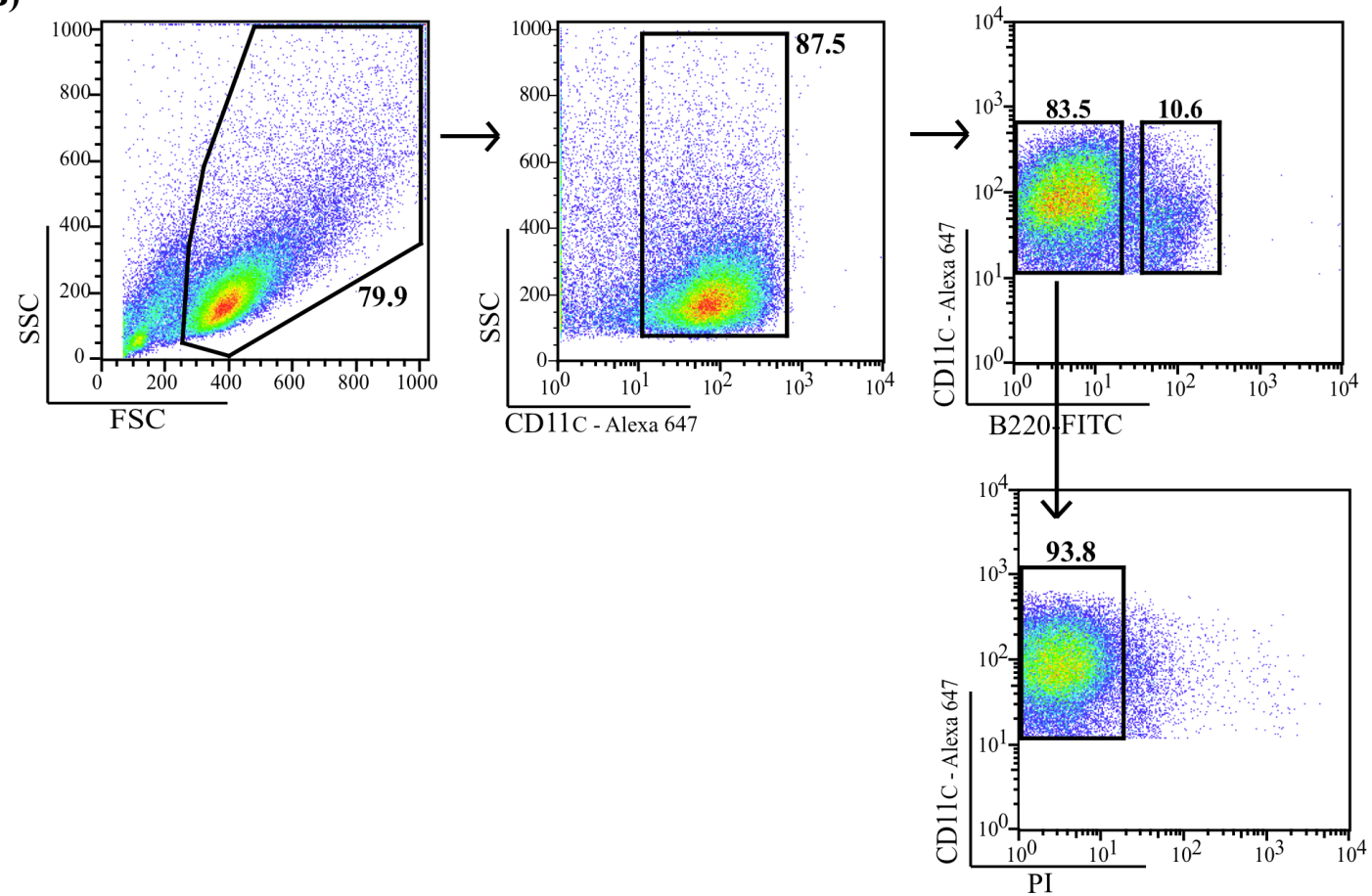

\section{Appendix 5: Identification of live Flt3L or GMCSF/IL-4 DCs by PI exclusion}

Flt3L and GMCSF/IL-4 DCs were generated as described in Chapter 2 (2.4.2.2). LPS stimulation occurred on day 9 for Flt3L-DCs and day 6 for GMCSF/IL-4 DCs at a final concentration of $100 \mathrm{ng} / \mathrm{mL}$ and $500 \mathrm{ng} / \mathrm{mL}$ for each DC population respectively. Following 6 hours of incubation, Flt3L-DCs were harvested and stained with various combinations of mAbs for the markers CD11c, B220, CD40, CD80 and MHC II expression. GMCSF/IL-4 DCs were stained with combinations of mAbs for markers CD11c, CD40, CD80 and MHC II. Both DC populations were subsequently stained with the viability dye PI and DCs were analysed by flow cytometry. Results show gating strategy and identification of live cells in Flt3L (A) and GMCSF/IL-4 DCs (B). Results are from one experiment only. 


\section{Appendix 6: Tumour size of individual mice vaccinated with varying numbers of Flt3L-cDCs or GMCSF/IL-4 DCs.}

BM cells extracted from C57BL/6 mice were cultured in the presence of Flt3L or GMCSF/IL-4. GMCSF/IL-4 cultures were set up 3 days following Flt3L cultures to allow for vaccination with each DC type to occur on the same day. On day 9 of cell culture, Flt3L-DCs were depleted of B220 $0^{+} \mathrm{pDCs}$, (Chapter 2, (2.4.3.3)), and Flt3L-cDCs were placed back into culture and stimulated with Pam3Cys/Poly I:C, at the

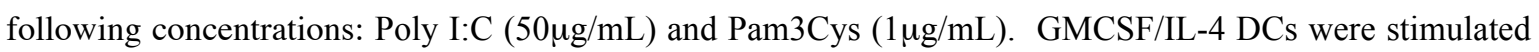
on day 6 of cell culture with LPS/Pam3Cys at the following concentrations: LPS $(100 \mathrm{n} / \mathrm{mL})$ and Pam3Cys $(100 \mathrm{ng} / \mathrm{mL})$. Following 24 hours of stimulation, DCs from each culture system were harvested and loaded with $10 \mu \mathrm{M}$ SIINFEKL peptide. DCs were titrated and varying numbers were injected s.c. into the right flank of C57BL/6 mice. All mice were injected s.c. into the left flank with $1 \times 10^{5}$ B16.OVA melanoma cells, 7 days post vaccination. A tumour was scored as positive when it reached $4 \mathrm{~mm}^{2}$. Tumour size was measured with Mitutoyo callipers and mice were euthanized when tumour size reached $150-200 \mathrm{~mm}^{2}$. (A) and (B) shows tumour size of each individual mouse vaccinated with varying numbers of Flt3L-cDCs or GMCSF/IL4 DCs, respectively. (C) shows tumour size of each mouse that received tumour only. 
A) Flt3L-cDCs
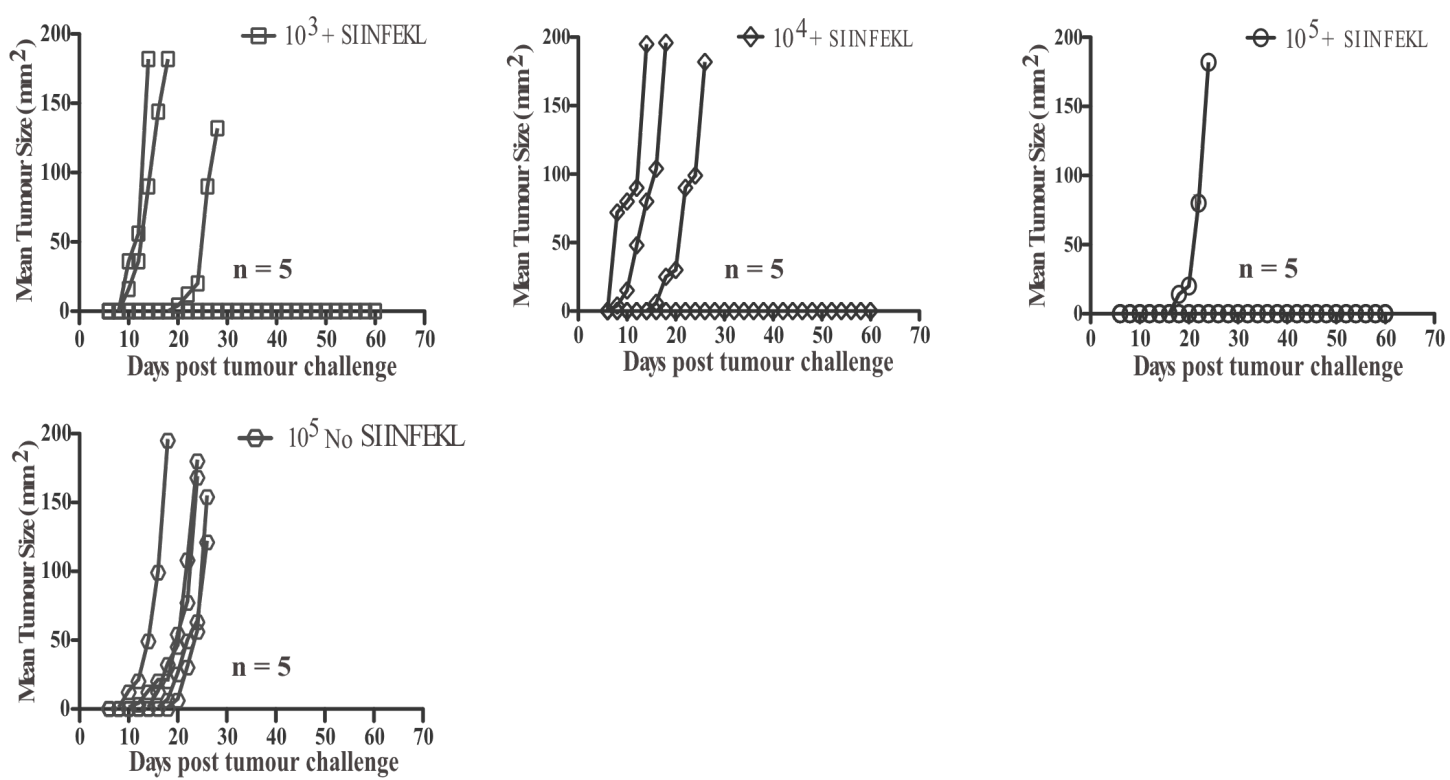

B) GMCSF/IL-4 DCs
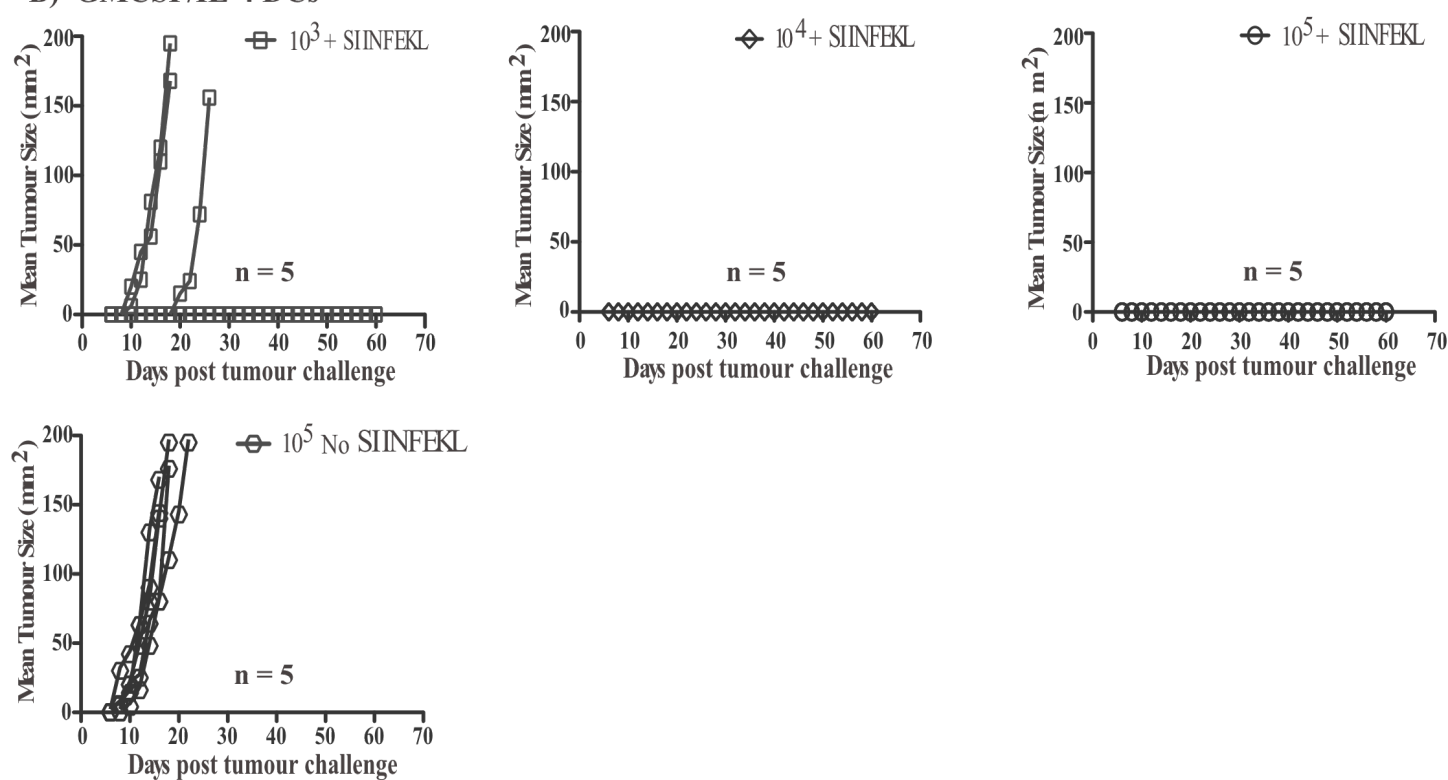

C) Tumour only control

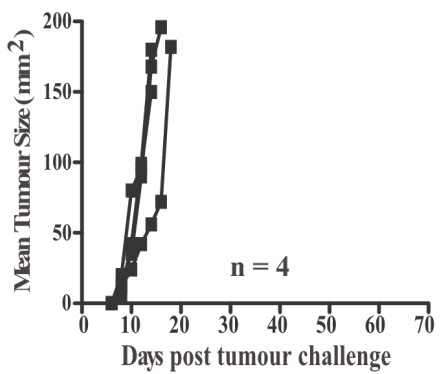




\section{Appendix 7: Tumour size of individual mice vaccinated with Flt3L-cDCs or GMCSF/IL-4 DCs, either stimulated or unstimulated}

BM cells extracted from C57BL/6 mice were cultured in the presence of Flt3L or GMCSF/IL-4. GMCSF/IL-4 cultures were set up 3 days following Flt3L cultures to allow for vaccination with each DC type to occur on the same day. On day 9 of cell culture, Flt3L-DCs were depleted of B220 pDCs, (Chapter 2, (2.4.3.3)), and Flt3L-cDCs were placed back into culture and stimulated with Pam3Cys/Poly I:C, at the following concentrations: Poly I:C (50 $\mu \mathrm{g} / \mathrm{mL})$ and Pam3Cys $(1 \mu \mathrm{g} / \mathrm{mL})$. GMCSF/IL-4 DCs were stimulated on day 6 of cell culture with LPS/Pam3Cys at the following concentrations: LPS $(100 \mathrm{n} / \mathrm{mL})$ and Pam3Cys $(100 \mathrm{ng} / \mathrm{mL})$. Following 24 hours of stimulation, DCs from each culture system were harvested and loaded with $10 \mu \mathrm{M}$ SIINFEKL peptide. DCs were titrated and varying numbers were injected s.c. into the right flank of C57BL/6 mice. All mice were injected s.c. into the left flank with $1 \times 10^{5}$ B16.OVA melanoma cells, 7 days post vaccination. A tumour was scored as positive when it reached $4 \mathrm{~mm}^{2}$. Tumour size was measured with Mitutoyo callipers and mice were euthanized when tumour size reached $150-200 \mathrm{~mm}^{2}$. (A) and (B) shows tumour size of each individual mouse vaccinated with Flt3L-cDCs or GMCSF/IL-4 DCs, respectively, either unstimulated or stimulated. (C) shows tumour size of each mouse that received tumour only. 
A) Flt3L-cDCs
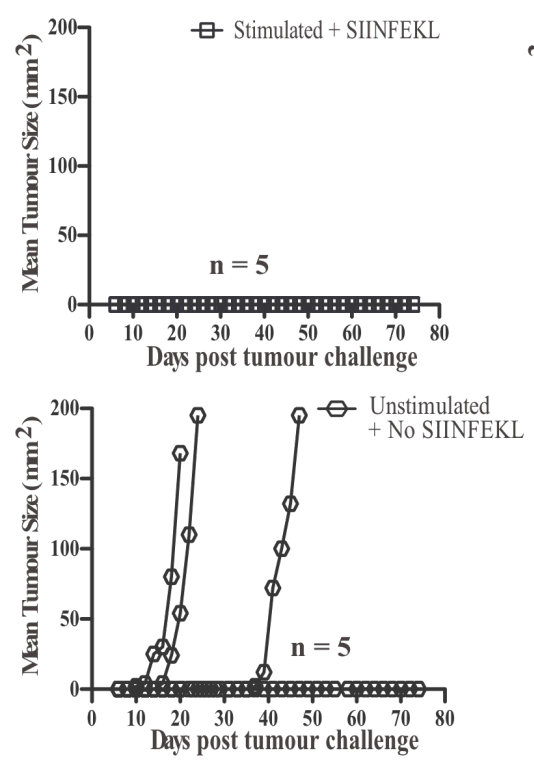

B) GMCSF/IL-4 DCs
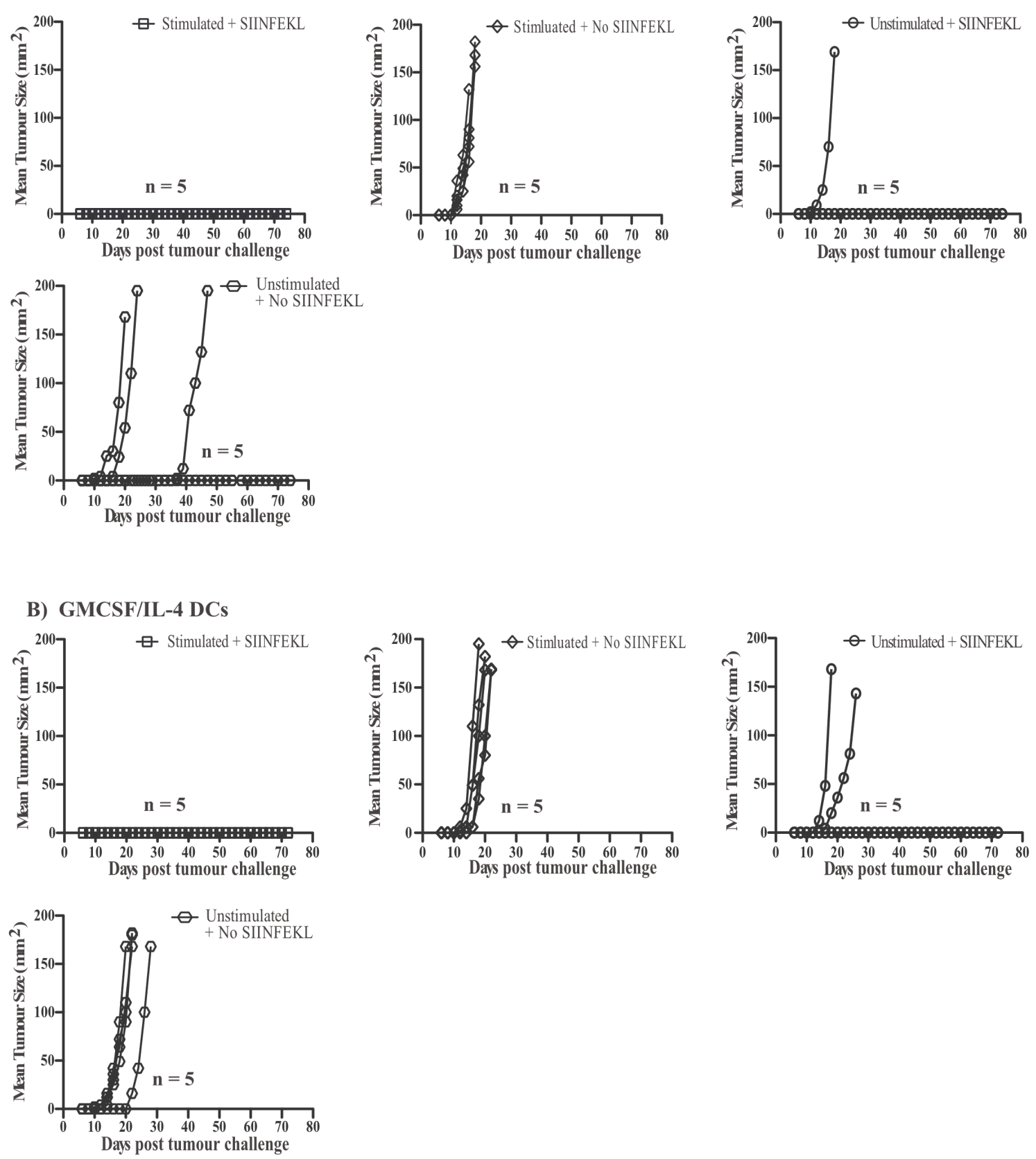

C) Tumour only control

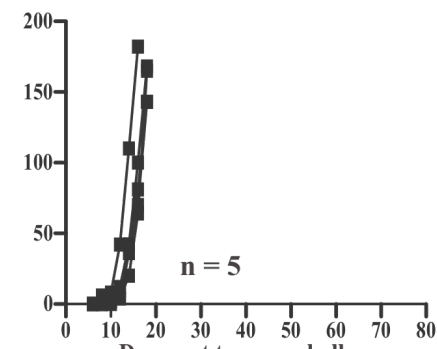

Days post tumour challenge 